\title{
Construction of Pyrrolo[1,2-a]indoles via Cobalt(III)-Catalyzed Enaminylation of 1-(Pyrimidin-2-yl)-1H-indoles with Ketenimines and Subsequent Base-Promoted Cyclization
}

\author{
Xiaorong Zhou, Zili Fan, Zhiyin Zhang, Ping Lu* and Yanguang Wang* \\ Department of Chemistry, Zhejiang University, P. R. China
}

\section{Content}

General considerations.....................................................

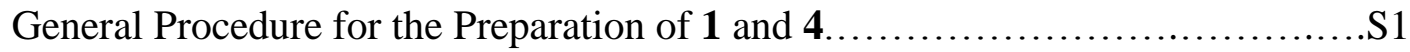

General Procedure for the Preparation of Ketenimines $2 \ldots \ldots \ldots \ldots \ldots \ldots \ldots \ldots$. 2

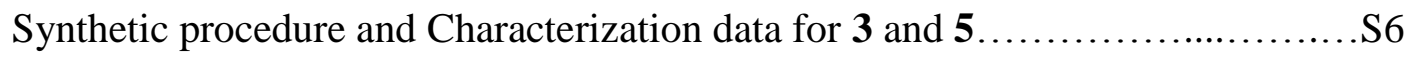

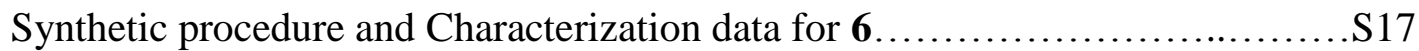

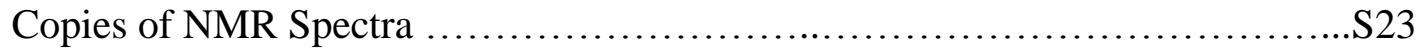

Scheme S1. Proposed Reaction Mechanism ................................S111 


\section{General considerations}

Unless otherwise mentioned, solvents and reagents were purchased from commercial sources and used as received. THF and toluene were distilled from $\mathrm{Na}$ before use. Solvents MeCN, $\mathrm{CH}_{2} \mathrm{Cl}_{2}$ and DCE were distilled from $\mathrm{CaH}_{2}$. Melting points were measured with a micro melting point apparatus. Infrared spectra were obtained with an FTIR spectrometer. NMR spectra were obtained at 500 or $400 \mathrm{MHz}$ for ${ }^{1} \mathrm{H}$ NMR, and 126 or $101 \mathrm{MHz}$ for ${ }^{13} \mathrm{C}$ NMR. Unless otherwise noted, all the NMR spectra were recorded at room temperature. Chemical shifts were quoted in parts per million ( $\mathrm{ppm}$ ) referenced to the appropriate solvent peak or $0.0 \mathrm{ppm}$ for tetramethylsilane (TMS) as an internal standard. The following abbreviations are used to describe peak patterns as appropriate: $\mathrm{s}=$ singlet, $\mathrm{d}=$ doublet, $\mathrm{t}=$ triplet, $\mathrm{q}=$ quartet, $\mathrm{m}=$ multiplet. Coupling constants $J$ were reported in hertz unit (Hz). High-resolution mass spectra (HRMS) data were obtained with an electron ionization time-of-flight (EI-TOF) or electrospray ionization (ESI) mass spectrometer. Flash column chromatography was performed employing 300-400 mesh silica gel. Thin layer chromatography (TLC) was performed on silica gel HSGF254.

\section{General Procedure for the Preparation of 1 and 4.}
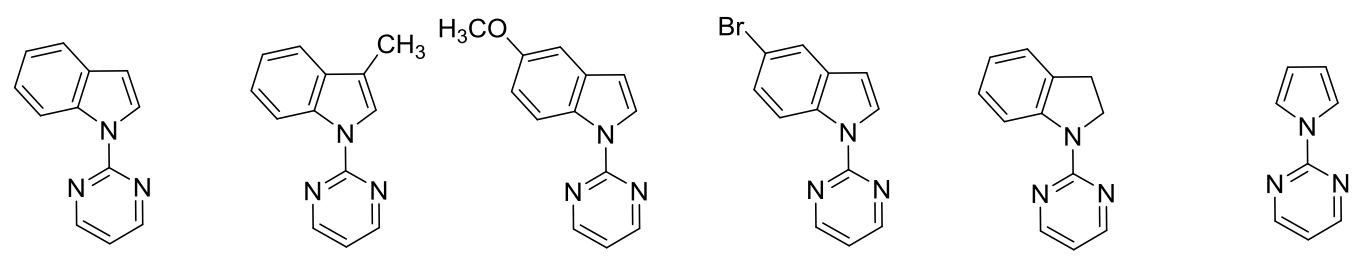

CAS: $221044-05-9$
1a<smiles>c1ccc(-c2ncccn2)cc1</smiles>

CAS: $7431-45-0$

$4 \mathbf{a}$

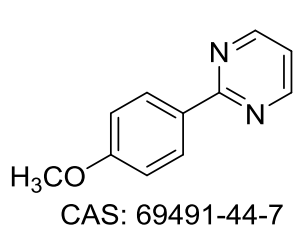

$4 b$<smiles>CC(=O)c1ccc(-c2ncccn2)cc1</smiles>

$4 c$<smiles>Cc1cccc(-c2ncccn2)c1</smiles>

CAS: 893641-03-7

4d<smiles>Cc1ccccc1-c1ncccn1</smiles>

CAS: 188527-65-3

Compounds 1a-1d, $1 \mathbf{1},{ }^{1} \mathbf{1} \mathbf{e}^{2}$ and $4 \mathbf{a}-4 \mathbf{e}^{3}$ were prepared according to the literature procedures.

\footnotetext{
${ }^{1}$ (1) Ackermann, L.; Lygin, A. V. Org. Lett. 2011, 13, 3332. (2) Xu, S.; Huang, X.; Hong, X.; Xu, B. Org. Lett. 2012, 14,4614

${ }^{2}$ Premi, C.; Dixit, A.; Jain, N. Org. Lett. 2015, 17, 2598.

${ }^{3}$ Song, B.; Zheng, X.; Mo, J.; Xu, B. Adv. Synth. Catal. 2010, 352, 329.
} 


\section{General Procedure for the Preparation of Ketenimines 2.}

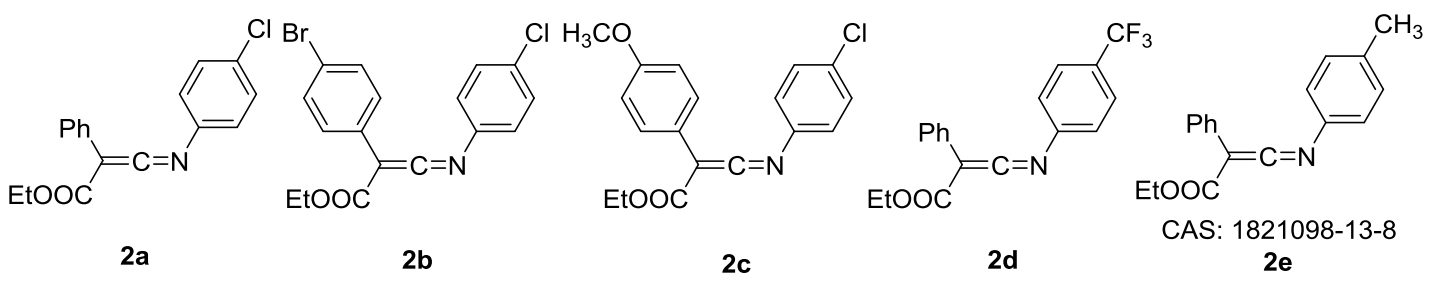

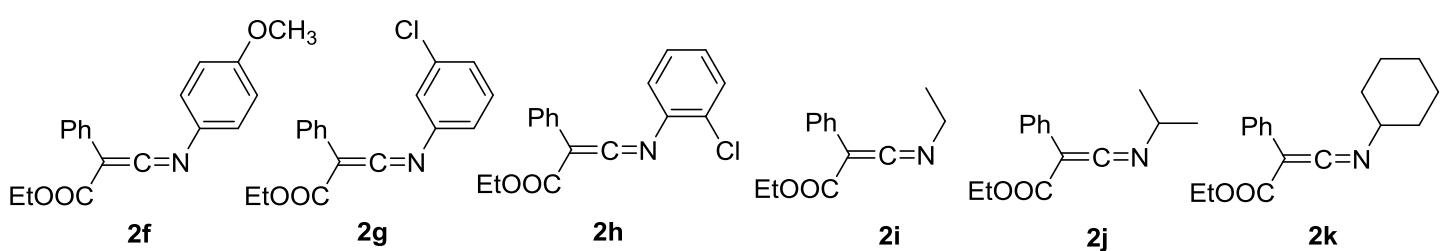

Compounds $2 \mathbf{e}$ as well as new products $\mathbf{2 a - 2 d}$ and $\mathbf{2 f - 2 k}$ were prepared according to the literature procedures ${ }^{4}$.

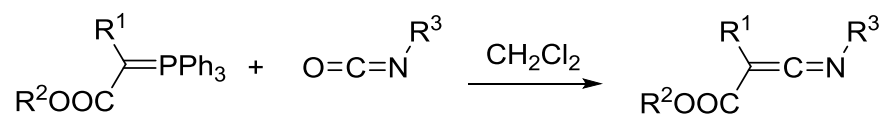

The following procedure is adapted from our previously reported method.

Method A (When $\mathrm{R}^{3}=$ aryl):

The mixture of ylide $\left(2 \mathrm{mmol}\right.$ ) and isocyanate $(2 \mathrm{mmol})$ in $5 \mathrm{~mL}$ of $\mathrm{CH}_{2} \mathrm{Cl}_{2}$ was stirred at reflux overnight. Then the solvent was removed in vacuum and the residue was purified by flash column chromatography on silica gel with petroleum ether/ethyl acetate $=20: 1(\mathrm{v}: \mathrm{v})$ as eluent.

Method B (When $\mathrm{R}^{3}=$ alkyl):

The mixture of ylide $(2 \mathrm{mmol})$ and isocyanate $(2 \mathrm{mmol})$ in $5 \mathrm{~mL}$ of $\mathrm{CH}_{2} \mathrm{Cl}_{2}$ was stirred at $80{ }^{\circ} \mathrm{C}$ in a pressure tube overnight. Then the solvent was removed in vacuum and the residue was purified by flash column chromatography on silica gel with petroleum ether as eluent.

General procedure for the preparation of corresponding phosphonium ylides.<smiles>[R]OCCc1ccc([R])cc1CC(OCC)c1ccc(C(=O)OCC)cc1</smiles>

Step 1: Following a modified procedure by reported lectures ${ }^{5}$. A mixture of corresponding ester ( 1 equiv), $\mathrm{N}$-bromosuccinide (1.2 equiv) and benzoyl peroxide $\left(20 \mathrm{mg}\right.$ ) in $\mathrm{CCl}_{4}$ was refluxed for $3 \mathrm{~h}$ and then allowed to stand overnight to precipitate any dissolved succinimide. The mixture was then filtered (CAUTION: bromine evolution!) and the filtrate was triturated with $\mathrm{CH}_{2} \mathrm{Cl}_{2}$. Then the organic phase was washed with statured $\mathrm{Na}_{2} \mathrm{~S}_{2} \mathrm{O}_{3}$ solution, saturated brine solution and dried over $\mathrm{Na}_{2} \mathrm{SO}_{4}$. The organic phase was then concentrated to give the desired product which was sufficiently pure to be used directly in the next reaction.

\footnotetext{
${ }^{4}$ Zhou, X.; Jiang, Z.; Xue, L.; Lu, P.; Wang, Y. Eur. J. Org. Chem. 2015, 2015, 5789.

5 (a) Sharma, V.; Tepe, J. J. Org. Lett. 2005, 7, 5091. (b) Lopchuk, J. M.; Hughes, R. P.; Gribble, G. W. Org. Lett. 2013, 15, 5218 .
} 
Step 2: To a stirred solution of NaI (1.5 equiv.) in acetone (c $1 \mathrm{M}$ ) was added $\alpha$-bromo ester (1 equiv.). The mixture was stirred at room temperature overnight. The solid was filtered off, and the solvent was distilled under reduce pressure. The residue was triturated with ether, and washed with statured $\mathrm{Na}_{2} \mathrm{~S}_{2} \mathrm{O}_{3}$ solution, saturated brine solution and dried over $\mathrm{Na}_{2} \mathrm{SO}_{4}$. Concentration under reduced pressure gave $\alpha$-iodo ester without any further purification.

Step 3: To a stirred solution of $\mathrm{PPh}_{3}$ (1 equiv.) in toluene (c $\left.0.5 \mathrm{M}\right)$ was added $\alpha$-iodo ester (1 equiv.) dropwise at room temperature. After the addition, the mixture was stirred at $60^{\circ} \mathrm{C}$ for 10 hours. The resulting salt was precipitated as a brown solid. The solid was filtered off, and washed with petroleum ether. The solid was triturated with $\mathrm{CH}_{2} \mathrm{Cl}_{2}$, and washed with $10 \% \mathrm{NaOH}$. The aq. phase was extracted twice with $\mathrm{CH}_{2} \mathrm{Cl}_{2}$. The organic phase was combined and concentrated under reduce pressure. The resulting phosphonium ylide was obtained and used directly for the subsequent reaction without further purification.

\section{Ethyl 3-((4-chlorophenyl)imino)-2-phenylacrylate (2a)}

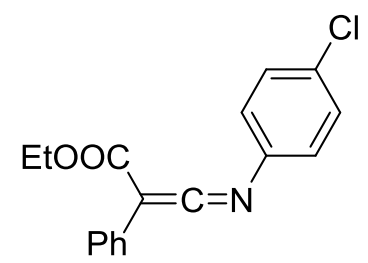

Following the general procedure (Method A) afforded 2a (389 $\mathrm{mg}, 65 \%$ ) as a pale yellow solid, m.p. $77-78^{\circ} \mathrm{C}$.

${ }^{1}$ H NMR (400 MHz, Chloroform- $d$ ) $\delta 7.56-7.50(\mathrm{~m}, 2 \mathrm{H}), 7.43-7.38(\mathrm{~m}, 2 \mathrm{H}), 7.37-7.32(\mathrm{~m}$, 2H), $7.32-7.27(\mathrm{~m}, 2 \mathrm{H}), 7.24-7.19(\mathrm{~m}, 1 \mathrm{H}), 4.30$ (q, $J=7.1 \mathrm{~Hz}, 2 \mathrm{H}), 1.30$ (t, $J=7.1 \mathrm{~Hz}, 3 \mathrm{H})$.

${ }^{13}$ C NMR (101 MHz, Chloroform- $d$ ) $\delta 181.7,167.4,135.5,134.5,130.0,129.7,128.7,127.5,126.6$, 125.7, 70.9, 60.7, 14.5.

IR (ATR): 2925, 2037, 1711, 1487, 1280, 1187, 1131, 1093, 1047, 834, $767 \mathrm{~cm}^{-1}$;

HRMS (EI-TOF) m/z calcd for $\mathrm{C}_{17} \mathrm{H}_{14} \mathrm{NO}_{2} \mathrm{Cl}$ [M $\left.{ }^{+}\right]$: 299.0713; found: 299.0716 .

Ethyl 2-(4-bromophenyl)-3-((4-chlorophenyl)imino)acrylate (2b)

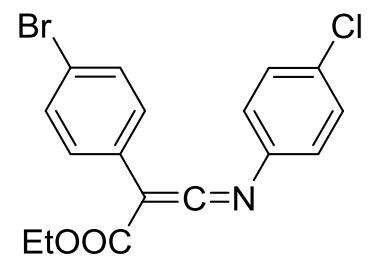

Following the general procedure (Method A) afforded $\mathbf{2 b}(475 \mathrm{mg}, 63 \%$ ) as a pale yellow solid, m.p. $103-104{ }^{\circ} \mathrm{C}$.

${ }^{1}$ H NMR (400 MHz, Chloroform- $d$ ) $\delta 7.48-7.38(\mathrm{~m}, 6 \mathrm{H}), 7.32-7.27(\mathrm{~m}, 2 \mathrm{H}), 4.29(\mathrm{q}, J=7.2 \mathrm{~Hz}$, $2 \mathrm{H}), 1.29(\mathrm{t}, J=7.1 \mathrm{~Hz}, 3 \mathrm{H})$.

${ }^{13}$ C NMR (101 MHz, Chloroform- $d$ ) $\delta$ 180.2, 167.1, 134.9, 134.7, 131.7, 130.0, 128.8, 125.8, 120.2, $70.0,60.9,14.4$.

IR (ATR): 2925, 2034, 1708, 1485, 1281, 1187, 1132, 1092, 1040, 1012, 829, $759 \mathrm{~cm}^{-1}$;

HRMS (EI-TOF) $\mathrm{m} / \mathrm{z}$ calcd for $\mathrm{C}_{17} \mathrm{H}_{13} \mathrm{NO}_{2} \mathrm{ClBr}\left[\mathrm{M}^{+}\right]: 376.9818$; found: 376.9814 .

Ethyl 3-((4-chlorophenyl)imino)-2-(4-methoxyphenyl)acrylate (2c) 


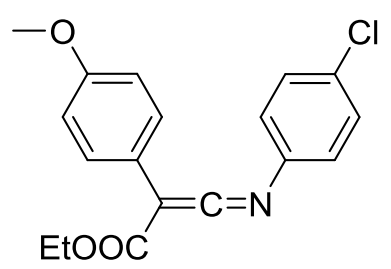

Following the general procedure (Method A) afforded 2c (447 mg, $68 \%$ ) as a pale yellow solid, m.p. $94-95^{\circ} \mathrm{C}$.

${ }^{1}$ H NMR (400 MHz, Chloroform- $\left.d\right) \delta 7.43(\mathrm{~d}, J=8.9 \mathrm{~Hz}, 2 \mathrm{H}), 7.39(\mathrm{~d}, J=8.7 \mathrm{~Hz}, 2 \mathrm{H}), 7.28(\mathrm{~d}, J$ $=8.7 \mathrm{~Hz}, 2 \mathrm{H}), 6.90(\mathrm{~d}, J=8.9 \mathrm{~Hz}, 2 \mathrm{H}), 4.29(\mathrm{q}, J=7.1 \mathrm{~Hz}, 2 \mathrm{H}), 3.80(\mathrm{~s}, 3 \mathrm{H}), 1.29$ (t, $J=7.1 \mathrm{~Hz}, 3 \mathrm{H})$.

${ }^{13}$ C NMR (101 MHz, Chloroform- $d$ ) $\delta$ 183.3, 167.7, 158.5, 135.9, 134.3, 129.9, 129.0, 125.6, 121.3, $114.2,70.6,60.7,55.3,14.4$.

IR (ATR): 2925, 2014, 1707, 1512, 1485, 1279, 1248, 1176, 1092, 1033, 831, $760 \mathrm{~cm}^{-1}$;

HRMS (EI-TOF) m/z calcd for $\mathrm{C}_{18} \mathrm{H}_{16} \mathrm{NO}_{3} \mathrm{Cl}\left[\mathrm{M}^{+}\right]$: 329.0819; found: 329.0816 .

\section{Ethyl 2-phenyl-3-((4-(trifluoromethyl)phenyl)imino)acrylate (2d)}

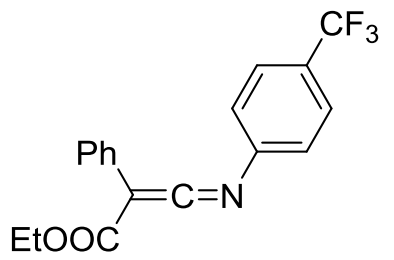

Following the general procedure (Method A) afforded 2d (420 mg, $63 \%$ ) as a yellow oil.

${ }^{1}$ H NMR (400 MHz, Chloroform- $d$ ) $\delta 7.70(\mathrm{~d}, J=8.3 \mathrm{~Hz}, 2 \mathrm{H}), 7.56-7.51(\mathrm{~m}, 2 \mathrm{H}), 7.44(\mathrm{~d}, J=8.2$ $\mathrm{Hz}, 2 \mathrm{H}), 7.39-7.33(\mathrm{~m}, 2 \mathrm{H}), 7.27-7.20(\mathrm{~m}, 1 \mathrm{H}), 4.32$ (q, $J=7.1 \mathrm{~Hz}, 2 \mathrm{H}), 1.32(\mathrm{t}, J=7.1 \mathrm{~Hz}, 3 \mathrm{H})$.

${ }^{13}$ C NMR (101 MHz, Chloroform- $\left.d\right) \delta 183.4,167.2,140.5,130.4(\mathrm{q}, J=33.1 \mathrm{~Hz}), 129.2,128.7$, 127.6, 127.0 (q, $J=3.7 \mathrm{~Hz}), 126.9,124.4,123.6$ (q, $J=270.4 \mathrm{~Hz}), 71.0,60.9$, 14.4.

IR (ATR): 2905, 2032, 1711, 1609, 1321, 1281, 1169, 1127, 1064, 1021, 845, 770, 738, 694, 666 $\mathrm{cm}^{-1}$.

HRMS (EI-TOF) m/z calcd for $\mathrm{C}_{18} \mathrm{H}_{14} \mathrm{NO}_{2} \mathrm{~F}_{3}\left[\mathrm{M}^{+}\right]$: 333.0977; found: 333.0979 .

\section{Ethyl 3-((4-methoxyphenyl)imino)-2-phenylacrylate (2f)}

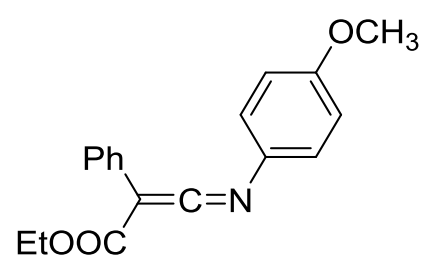

Following the general procedure (Method A) afforded $2 \mathbf{f}(407 \mathrm{mg}, 69 \%)$ as a pale yellow solid, m.p. $66-67^{\circ} \mathrm{C}$.

${ }^{1}$ H NMR (400 MHz, Chloroform- $\left.d\right) \delta 7.58-7.53(\mathrm{~m}, 2 \mathrm{H}), 7.36-7.29(\mathrm{~m}, 4 \mathrm{H}), 7.21-7.15(\mathrm{~m}$, $1 \mathrm{H}), 6.96-6.90(\mathrm{~m}, 2 \mathrm{H}), 4.28(\mathrm{q}, J=7.1 \mathrm{~Hz}, 2 \mathrm{H}), 3.83(\mathrm{~s}, 3 \mathrm{H}), 1.29(\mathrm{t}, J=7.1 \mathrm{~Hz}, 3 \mathrm{H})$.

${ }^{13}$ C NMR (101 MHz, Chloroform- $d$ ) $\delta 178.1,167.9,159.9,130.6,128.9,128.5,127.3,126.3$, $126.1,114.9,70.1,60.5,55.6,14.5$.

IR (ATR): 2981, 2027, 1704, 1503, 1278, 1254, 1185, 1129, 1029, 835, 765, $751 \mathrm{~cm}^{-1}$.

HRMS (EI-TOF) m/z calcd for $\mathrm{C}_{18} \mathrm{H}_{17} \mathrm{NO}_{3}\left[\mathrm{M}^{+}\right]$: 295.1208; found: 295.1206 . 
Ethyl 3-((3-chlorophenyl)imino)-2-phenylacrylate (2g)

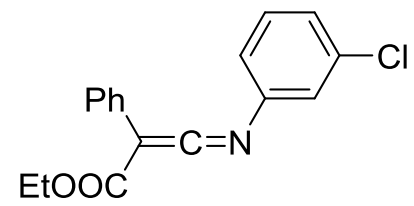

Following the general procedure (Method A) afforded $2 \mathrm{~g}$ ( $335 \mathrm{mg}, 56 \%$ ) as a yellow oil.

${ }^{1}$ H NMR $(400 \mathrm{MHz}$, Chloroform- $d$ ) $\delta 7.57-7.50(\mathrm{~m}, 2 \mathrm{H}), 7.39-7.31(\mathrm{~m}, 5 \mathrm{H}), 7.27-7.19(\mathrm{~m}$, 2H), 4.31 (q, $J=7.1 \mathrm{~Hz}, 2 \mathrm{H}), 1.31(\mathrm{t}, J=7.1 \mathrm{~Hz}, 3 \mathrm{H})$.

${ }^{13}$ C NMR (101 MHz, Chloroform- $d$ ) $\delta$ 182.5, 167.3, 138.3, 135.3, 130.7, 129.5, 128.7, 127.5, 126.7, $124.5,122.5,70.9,60.8,14.4$.

IR (ATR): 2982, 2024, 1710, 1585, 1472, 1284, 1185, 1138, 1046, 870, 771, $690 \mathrm{~cm}^{-1}$.

HRMS (EI-TOF) m/z calcd for $\mathrm{C}_{17} \mathrm{H}_{14} \mathrm{NO}_{2} \mathrm{Cl}\left[\mathrm{M}^{+}\right]$: 299.0713; found: 299.0710 .

Ethyl 3-((2-chlorophenyl)imino)-2-phenylacrylate (2h)

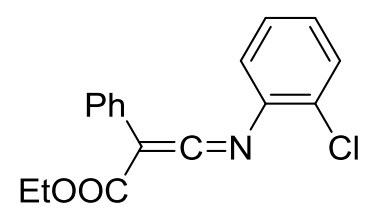

Following the general procedure (Method A) afforded $2 \mathbf{h}(287 \mathrm{mg}, 48 \%)$ as a yellow oil.

${ }^{1}$ H NMR (400 MHz, Chloroform- $d$ ) $\delta 7.61-7.55(\mathrm{~m}, 2 \mathrm{H}), 7.51-7.47(\mathrm{~m}, 1 \mathrm{H}), 7.40-7.26(\mathrm{~m}$, $5 \mathrm{H}), 7.24-7.18(\mathrm{~m}, 1 \mathrm{H}), 4.31(\mathrm{q}, J=7.1 \mathrm{~Hz}, 2 \mathrm{H}), 1.32(\mathrm{t}, J=7.1 \mathrm{~Hz}, 3 \mathrm{H})$.

${ }^{13}$ C NMR (101 MHz, Chloroform- $d$ ) $\delta$ 180.7, 167.6, 134.4, 130.6, 129.9, 129.7, 129.3, 128.6, 127.9, $127.5,126.4,125.8,69.5,60.7,14.5$.

HRMS (EI-TOF) m/z calcd for $\mathrm{C}_{17} \mathrm{H}_{14} \mathrm{NO}_{2} \mathrm{Cl}\left[\mathrm{M}^{+}\right]$: 299.0713; found: 299.0713.

\section{Ethyl 3-(ethylimino)-2-phenylacrylate (2i)}

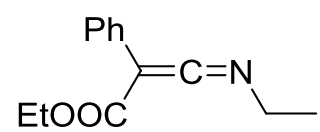

Following the general procedure (Method B) afforded $2 \mathbf{i}(208 \mathrm{mg}, 48 \%)$ as a colorless oil.

${ }^{1}$ H NMR $(400 \mathrm{MHz}$, Chloroform- $d$ ) $\delta 7.53-7.47$ (m, 2H), $7.35-7.29(\mathrm{~m}, 2 \mathrm{H}), 7.18-7.15(\mathrm{~m}, 1 \mathrm{H})$, 4.25 (q, $J=7.1 \mathrm{~Hz}, 2 \mathrm{H}), 3.80$ (q, $J=7.2 \mathrm{~Hz}, 2 \mathrm{H}), 1.44(\mathrm{t}, J=7.2 \mathrm{~Hz}, 3 \mathrm{H}), 1.30(\mathrm{t}, J=7.1 \mathrm{~Hz}, 3 \mathrm{H})$.

${ }^{13}$ C NMR (101 MHz, Chloroform- $d$ ) $\delta$ 172.4, 168.6, 131.2, 128.4, 126.9, 125.6, 67.9, 60.1, 46.1, 15.7, 14.4.

IR (film): 2981, 2055, 1698, 1598, 1497, 1448, 1337, 1290, 1156, 1050, 770, $694 \mathrm{~cm}^{-1}$;

HRMS (EI-TOF) $\mathrm{m} / \mathrm{z}$ calcd for $\mathrm{C}_{13} \mathrm{H}_{15} \mathrm{NO}_{2}\left[\mathrm{M}^{+}\right]$: 217.1103; found: 217.1103 .

\section{Ethyl 3-(isopropylimino)-2-phenylacrylate (2j)}

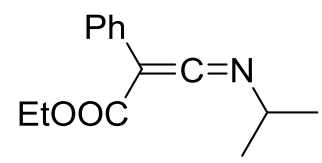

Following the general procedure (Method B) afforded $\mathbf{2 j}$ ( $259 \mathrm{mg}, 56 \%$ ) as a colorless oil. 
${ }^{1}$ H NMR (400 MHz, Chloroform- $d$ ) $\delta 7.54-7.48(\mathrm{~m}, 2 \mathrm{H}), 7.35$ - $7.28(\mathrm{~m}, 2 \mathrm{H}), 7.18$ - $7.11(\mathrm{~m}$, $1 \mathrm{H}), 4.24(\mathrm{q}, J=7.1 \mathrm{~Hz}, 2 \mathrm{H}), 4.12$ (hept, $J=6.5 \mathrm{~Hz}, 1 \mathrm{H}), 1.43(\mathrm{~d}, J=6.5 \mathrm{~Hz}, 6 \mathrm{H}), 1.30(\mathrm{t}, J=7.1 \mathrm{~Hz}$, $3 \mathrm{H})$.

${ }^{13}$ C NMR (101 MHz, Chloroform- $d$ ) $\delta$ 171.6, 168.6, 131.3, 128.4, 126.7, 125.5, 68.3, 60.0, 54.9, 23.6, 14.4.

IR (film): 2978, 2933, 2049, 1699, 1598, 1495, 1450, 1367, 1286, 1155, 1101, 1049, 1029, 771, $694,661 \mathrm{~cm}^{-1}$;

HRMS (EI-TOF) m/z calcd for $\mathrm{C}_{14} \mathrm{H}_{17} \mathrm{NO}_{2}\left[\mathrm{M}^{+}\right]$: 231.1259; found: 231.1259 .

\section{Ethyl 3-(cyclohexylimino)-2-phenylacrylate (2k)}

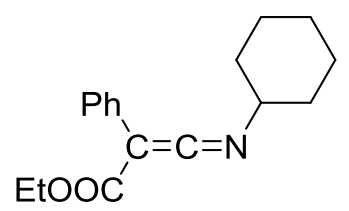

Following the general procedure (Method B) afforded $\mathbf{2 j}(217 \mathrm{mg}, 40 \%)$ as a colorless oil.

${ }^{1}$ H NMR $(400 \mathrm{MHz}$, Chloroform- $d) \delta 7.52(\mathrm{~d}, J=7.4 \mathrm{~Hz}, 2 \mathrm{H}), 7.31(\mathrm{t}, J=7.7 \mathrm{~Hz}, 2 \mathrm{H}), 7.13(\mathrm{t}, J=$ $7.4 \mathrm{~Hz}, 1 \mathrm{H}), 4.24$ (q, $J=7.1 \mathrm{~Hz}, 2 \mathrm{H}), 3.91-3.84(\mathrm{~m}, 1 \mathrm{H}), 2.11-2.02(\mathrm{~m}, 2 \mathrm{H}), 1.84-1.78(\mathrm{~m}, 2 \mathrm{H})$, $1.68-1.56(\mathrm{~m}, 3 \mathrm{H}), 1.45-1.33(\mathrm{~m}, 3 \mathrm{H}), 1.30(\mathrm{t}, J=7.1 \mathrm{~Hz}, 3 \mathrm{H})$.

${ }^{13}$ C NMR (101 MHz, Chloroform- $\left.d\right) \delta$ 170.2, 168.7, 131.5, 128.4, 126.6, 125.4, 67.3, 61.0, 60.0, 33.6, 25.2, 23.9, 14.4 .

IR (ATR): 2933, 2047, 1700, 1559, 1496, 1449, 1285, 1153, 1050, 769, $694 \mathrm{~cm}^{-1}$.

HRMS (EI-TOF) $\mathrm{m} / \mathrm{z}$ calcd for $\mathrm{C}_{17} \mathrm{H}_{21} \mathrm{NO}_{2}\left[\mathrm{M}^{+}\right]$: 271.1572; found: 271.1576

\section{Synthetic procedure and Characterization data for 3 and 5}

\section{Typical procedure for the preparation of Ethyl (Z)-3-((4-chlorophenyl)amino)-2-phenyl-3-(1-} (pyrimidin-2-yl)-1H-indol-2-yl)acrylate (3a)
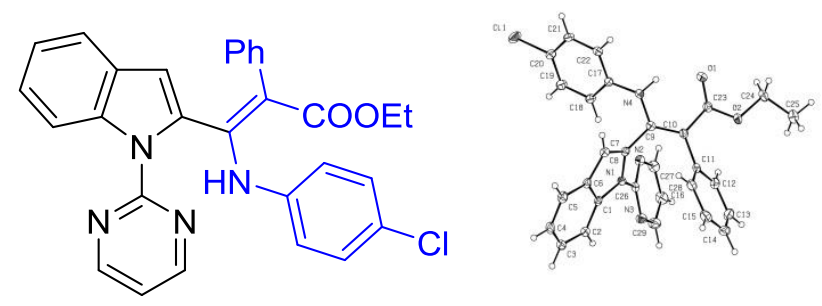

To an oven-dried Schlenk tube equipped with a magnetic stirring bar were added sequentially 1a (0.2 mmol), 2a (0.25 mmol), [Cp*Co(CO) $\left.\mathrm{I}_{2}\right](0.005 \mathrm{mmol}), \operatorname{AgNTf}_{2}(0.015 \mathrm{mmol})$, and dry DCE (2 $\mathrm{mL}$ ) under $\mathrm{N}_{2}$ atmosphere. The reaction vessel was heated to $80{ }^{\circ} \mathrm{C}$ in oil bath for 10 hours. The reaction mixture was cooled to room temperature and then the solvent was evaporated in vacuum. The residue was purified by column chromatography on silica gel (petroleum ether/ethyl acetate $=6: 1$ ) to give pure product 3a (93 mg, $94 \%$ ) as a pale yellow solid; m.p. $200-202{ }^{\circ} \mathrm{C}$.

${ }^{1}$ H NMR $\left(400 \mathrm{MHz}, \mathrm{CDCl}_{3}\right) \delta 11.27(\mathrm{~s}, 1 \mathrm{H}), 8.62(\mathrm{~d}, J=4.8 \mathrm{~Hz}, 2 \mathrm{H}), 8.16(\mathrm{dd}, J=8.4,0.9 \mathrm{~Hz}$, $1 \mathrm{H}), 7.43(\mathrm{~d}, J=7.5 \mathrm{~Hz}, 1 \mathrm{H}), 7.21$ (ddd, $J=8.4,7.0,1.3 \mathrm{~Hz}, 1 \mathrm{H}), 7.13(\mathrm{ddd}, J=8.1,7.2,1.1 \mathrm{~Hz}, 1 \mathrm{H}$ ), $7.08(\mathrm{t}, J=4.8 \mathrm{~Hz}, 1 \mathrm{H}), 6.98-6.84(\mathrm{~m}, 5 \mathrm{H}), 6.78-6.72(\mathrm{~m}, 2 \mathrm{H}), 6.70-6.63(\mathrm{~m}, 2 \mathrm{H}), 6.46(\mathrm{~d}, J=0.8$ $\mathrm{Hz}, 1 \mathrm{H}), 4.20-4.14(\mathrm{~m}, 2 \mathrm{H}), 1.19$ (t, $J=7.1 \mathrm{~Hz}, 3 \mathrm{H})$. 
${ }^{13} \mathbf{C}$ NMR $\left(101 \mathrm{MHz}, \mathrm{CDCl}_{3}\right) \delta 170.2,157.6,156.4,150.8,139.1,136.5,135.8,131.4,131.2,128.5$, 128.3, 128.1, 126.9, 125.6, 124.4, 122.7, 122.1, 121.1, 117.0, 114.4, 112.4, 102.2, 59.9, 14.3.

IR (ATR): 2982, 1731, 1645, 1595, 1568, 1492, 1429, 1242, 1205, 1092, 1046, 818, 795, 748, 701 $\mathrm{cm}^{-1}$.

HRMS (EI-TOF) m/z calcd for $\mathrm{C}_{29} \mathrm{H}_{23} \mathrm{~N}_{4} \mathrm{O}_{2} \mathrm{Cl}\left[\mathrm{M}^{+}\right]$: 494.1510; found: 494.1524 .

Ethyl

(Z)-3-((4-chlorophenyl)amino)-3-(3-methyl-1-(pyrimidin-2-yl)-1H-indol-2-yl)-2phenylacrylate (3b)<smiles>CCOC(=O)/C(=C(\Nc1ccc(Cl)cc1)c1c(C)c2ccccc2n1-c1ncccn1)c1ccccc1</smiles>

The reaction of $\mathbf{1 b}(0.2 \mathrm{mmol}), \mathbf{2 a}(0.25 \mathrm{mmol})$, [Cp*Co(CO) $\left.\mathrm{I}_{2}\right](0.005 \mathrm{mmol}), \operatorname{AgNTf}_{2}(0.015$ $\mathrm{mmol})$ in DCE (2 mL) following the typical procedure afforded $\mathbf{3 b}(73 \mathrm{mg}, 72 \%)$ after chromatography on silica gel (petroleum ether/ethyl acetate $=6: 1$ ) as a pale yellow solid; m.p. 180 $184^{\circ} \mathrm{C}$.

${ }^{1} \mathbf{H}$ NMR $\left(400 \mathrm{MHz}, \mathrm{CDCl}_{3}\right) \delta 11.44(\mathrm{~s}, 1 \mathrm{H}), 8.61(\mathrm{~d}, J=4.8 \mathrm{~Hz}, 2 \mathrm{H}), 8.20(\mathrm{~d}, J=8.3 \mathrm{~Hz}, 1 \mathrm{H})$, $7.39(\mathrm{~d}, J=7.5 \mathrm{~Hz}, 1 \mathrm{H}), 7.22(\mathrm{ddd}, J=8.4,7.1,1.4 \mathrm{~Hz}, 1 \mathrm{H}), 7.18-7.11(\mathrm{~m}, 1 \mathrm{H}), 7.05(\mathrm{t}, J=4.8 \mathrm{~Hz}$, $1 \mathrm{H}), 6.95-6.86(\mathrm{~m}, 3 \mathrm{H}), 6.84-6.74(\mathrm{~m}, 4 \mathrm{H}), 6.62-6.54(\mathrm{~m}, 2 \mathrm{H}), 4.22-4.11(\mathrm{~m}, 2 \mathrm{H}), 2.06(\mathrm{~s}, 3 \mathrm{H})$, $1.18(\mathrm{t}, J=7.1 \mathrm{~Hz}, 3 \mathrm{H})$.

${ }^{13} \mathbf{C}$ NMR $\left(101 \mathrm{MHz}, \mathrm{CDCl}_{3}\right) \delta 170.1,157.5,156.5,151.0,139.0,136.2,135.7,130.6,129.2,128.5$, 128.1, 127.8, 126.7, 125.6, 124.5, 122.3, 121.7, 119.1, 117.8, 116.5, 114.5, 102.2, 59.8, 14.3, 9.1.

IR (ATR): 2982, 1642, 1558, 1492, 1430, 1261, 1196, 1096, 908, 748, $700 \mathrm{~cm}^{-1}$.

HRMS (EI-TOF) m/z calcd for $\mathrm{C}_{30} \mathrm{H}_{25} \mathrm{~N}_{4} \mathrm{O}_{2} \mathrm{Cl}\left[\mathrm{M}^{+}\right]$: 508.1666; found: 508.1672 .

Ethyl (Z)-3-((4-chlorophenyl)amino)-3-(5-methoxy-1-(pyrimidin-2-yl)-1H-indol-2-yl)-2phenylacrylate (3c)<smiles>CCOC(=O)C(=C(Nc1ccc(Cl)cc1)c1cc2cc(OC)ccc2n1-c1ncccn1)c1ccccc1</smiles>

The reaction of $\mathbf{1 c}(0.2 \mathrm{mmol}), \mathbf{2 a}(0.25 \mathrm{mmol}),\left[\mathrm{Cp} * \mathrm{Co}(\mathrm{CO}) \mathrm{I}_{2}\right](0.005 \mathrm{mmol}), \operatorname{AgNTf}_{2}(0.015$ $\mathrm{mmol})$ in DCE (2 mL) following the typical procedure afforded $\mathbf{3 c}(80 \mathrm{mg}, 76 \%)$ after chromatography on silica gel (petroleum ether/ethyl acetate $=6: 1$ ) as a pale yellow solid; m.p. $180-$ $184{ }^{\circ} \mathrm{C}$.

${ }^{1} \mathbf{H}$ NMR $\left(400 \mathrm{MHz}, \mathrm{CDCl}_{3}\right) \delta 11.26(\mathrm{~s}, 1 \mathrm{H}), 8.59(\mathrm{~d}, J=4.8 \mathrm{~Hz}, 2 \mathrm{H}), 8.08(\mathrm{~d}, J=9.0 \mathrm{~Hz}, 1 \mathrm{H})$, $7.05(\mathrm{t}, J=4.8 \mathrm{~Hz}, 1 \mathrm{H}), 6.97-6.81(\mathrm{~m}, 7 \mathrm{H}), 6.78-6.73(\mathrm{~m}, 2 \mathrm{H}), 6.68-6.63(\mathrm{~m}, 2 \mathrm{H}), 6.38(\mathrm{~s}, 1 \mathrm{H})$, $4.20-4.13$ (m, 2H), 3.79 (s, 3H), $1.19(\mathrm{t}, J=7.1 \mathrm{~Hz}, 3 \mathrm{H})$.

${ }^{13} \mathbf{C}$ NMR $\left(101 \mathrm{MHz}, \mathrm{CDCl}_{3}\right) \delta 170.2,157.5,156.3,155.3,150.9,139.1,136.5,131.8,131.1,130.8$, 129.0, 128.5, 128.0, 126.8, 125.5, 122.7, 116.8, 115.4, 114.0, 112.1, 102.6, 102.1, 59.9, 55.4, 14.3. 
IR (ATR): 2985, 1643, 1569, 1431, 1268, 1213, 1166, 1093, 1045, 910, $701 \mathrm{~cm}^{-1}$.

HRMS (ESI) $\mathrm{m} / \mathrm{z}$ calcd for $\mathrm{C}_{30} \mathrm{H}_{25} \mathrm{~N}_{4} \mathrm{O}_{3} \mathrm{ClNa}^{+}[\mathrm{M}+\mathrm{H}]^{+}:$547.1507; found: 547.1480.

Ethyl

(Z)-3-(5-bromo-1-(pyrimidin-2-yl)-1H-indol-2-yl)-3-((4-chlorophenyl)amino)-2phenylacrylate $(3 d)$<smiles>CCOC(=O)/C(=C(\Nc1ccc(Cl)cc1)c1cc2cc(Br)ccc2n1-c1ncccn1)c1ccccc1</smiles>

The reaction of $\mathbf{1 d}(0.2 \mathrm{mmol}), \mathbf{2 a}(0.25 \mathrm{mmol}),\left[\mathrm{Cp} * \mathrm{Co}(\mathrm{CO}) \mathrm{I}_{2}\right](0.005 \mathrm{mmol}), \operatorname{AgNTf}_{2}(0.015$ $\mathrm{mmol})$ in DCE $(2 \mathrm{~mL})$ following the typical procedure afforded 3d $(80 \mathrm{mg}, 70 \%)$ after chromatography on silica gel (petroleum ether/ethyl acetate $=6: 1$ ) as a pale yellow solid; m.p. $210-$ $213^{\circ} \mathrm{C}$.

${ }^{1} \mathbf{H}$ NMR $\left(400 \mathrm{MHz}, \mathrm{CDCl}_{3}\right) \delta 11.23(\mathrm{~s}, 1 \mathrm{H}), 8.62(\mathrm{~d}, J=4.8 \mathrm{~Hz}, 2 \mathrm{H}), 8.05(\mathrm{~d}, J=8.9 \mathrm{~Hz}, 1 \mathrm{H})$, $7.55(\mathrm{~d}, J=1.9 \mathrm{~Hz}, 1 \mathrm{H}), 7.27(\mathrm{dd}, J=8.9,2.0 \mathrm{~Hz}, 1 \mathrm{H}), 7.11(\mathrm{t}, J=4.8 \mathrm{~Hz}, 1 \mathrm{H}), 6.99-6.85(\mathrm{~m}, 5 \mathrm{H})$, $6.77-6.71(\mathrm{~m}, 2 \mathrm{H}), 6.67-6.61(\mathrm{~m}, 2 \mathrm{H}), 6.38(\mathrm{~s}, 1 \mathrm{H}), 4.20-4.13(\mathrm{~m}, 2 \mathrm{H}), 1.19(\mathrm{t}, J=7.1 \mathrm{~Hz}, 3 \mathrm{H})$.

${ }^{13}$ C NMR $\left(101 \mathrm{MHz}, \mathrm{CDCl}_{3}\right) \delta 170.1,157.7,156.1,150.3,138.9,136.3,134.4,132.6,131.1,129.9$, 128.6, 128.3, 127.1, 126.9, 125.7, 123.5, 122.7, 117.4, 116.1, 115.4, 111.3, 102.5, 60.0, 14.3.

IR (ATR): 2927, 1644, 1595, 1568, 1492, 1427, 1261, 1208, 1132, 1092, 1046, 909, 805, 732, 701 $\mathrm{cm}^{-1}$.

HRMS (ESI) m/z calcd for $\mathrm{C}_{29} \mathrm{H}_{23} \mathrm{~N}_{4} \mathrm{O}_{2} \mathrm{ClBr}^{+}[\mathrm{M}+\mathrm{H}]^{+}:$: 573.0687; found: 573.0676 .

Ethyl (Z)-2-(4-bromophenyl)-3-((4-chlorophenyl)amino)-3-(1-(pyrimidin-2-yl)-1H-indol-2-yl) acrylate (3e)<smiles>CCOC(=O)/C(=C(\Nc1ccc(Cl)cc1)c1cc2ccccc2n1-c1ncccn1)c1ccc(Br)cc1</smiles>

The reaction of $\mathbf{1 a}(0.2 \mathrm{mmol}), \mathbf{2 b}(0.25 \mathrm{mmol}),\left[\mathrm{Cp} * \mathrm{Co}(\mathrm{CO}) \mathrm{I}_{2}\right](0.005 \mathrm{mmol}), \operatorname{AgNTf}_{2}(0.015$ mmol) in DCE $(2 \mathrm{~mL})$ following the typical procedure afforded 3e (109 $\mathrm{mg}$, $95 \%)$ after chromatography on silica gel (petroleum ether/ethyl acetate $=6: 1$ ) as a pale yellow solid; m.p. 212 $214{ }^{\circ} \mathrm{C}$.

${ }^{1} \mathbf{H}$ NMR $\left(400 \mathrm{MHz}, \mathrm{CDCl}_{3}\right) \delta 11.21(\mathrm{~s}, 1 \mathrm{H}), 8.62(\mathrm{~d}, J=4.8 \mathrm{~Hz}, 2 \mathrm{H}), 8.20(\mathrm{~d}, J=8.4 \mathrm{~Hz}, 1 \mathrm{H})$, $7.45(\mathrm{~d}, J=7.7 \mathrm{~Hz}, 1 \mathrm{H}), 7.28-7.21(\mathrm{~m}, 1 \mathrm{H}), 7.18-7.14(\mathrm{~m}, 1 \mathrm{H}), 7.08(\mathrm{t}, J=4.8 \mathrm{~Hz}, 1 \mathrm{H}), 7.04(\mathrm{~d}, J=$ $8.5 \mathrm{~Hz}, 2 \mathrm{H}), 6.91-6.85(\mathrm{~m}, 2 \mathrm{H}), 6.77(\mathrm{~d}, J=8.4 \mathrm{~Hz}, 2 \mathrm{H}), 6.55-6.49(\mathrm{~m}, 2 \mathrm{H}), 6.42(\mathrm{~s}, 1 \mathrm{H}), 4.27-$ $4.08(\mathrm{~m}, 2 \mathrm{H}), 1.21(\mathrm{t}, J=7.1 \mathrm{~Hz}, 3 \mathrm{H})$. 
${ }^{13}$ C NMR $\left(101 \mathrm{MHz}, \mathrm{CDCl}_{3}\right) \delta 169.87,157.65,156.35,151.09,138.60,135.93,135.88,133.04$, $130.95,130.06,128.52,128.35,128.16,124.59,122.59,122.27,121.20,119.61,117.06,114.60$, $112.82,100.91,59.99,14.33$.

IR (ATR): 2982, 1644, 1595, 1568, 1488, 1429, 1312, 1272, 1203, 1129, 1093, 1046, 1012, 907, $821,731 \mathrm{~cm}^{-1}$.

HRMS (ESI) $\mathrm{m} / \mathrm{z}$ calcd for $\mathrm{C}_{29} \mathrm{H}_{23} \mathrm{~N}_{4} \mathrm{O}_{2} \mathrm{ClBr}[\mathrm{M}+\mathrm{H}]^{+}:$573.0687; found: 573.0666 .

Ethyl (Z)-3-((4-chlorophenyl)amino)-2-(4-methoxyphenyl)-3-(1-(pyrimidin-2-yl)-1H-indol-2-yl) acrylate (3f)<smiles>CCOC(=O)/C(=C(\Nc1ccc(Cl)cc1)c1cc2ccccc2n1-c1ncccn1)c1ccc(OC)cc1</smiles>

The reaction of 1a $(0.2 \mathrm{mmol}), \mathbf{2 c}(0.25 \mathrm{mmol}),\left[\mathrm{Cp} * \mathrm{Co}(\mathrm{CO}) \mathrm{I}_{2}\right](0.005 \mathrm{mmol}), \operatorname{AgNTf}_{2}(0.015$ $\mathrm{mmol})$ in DCE $(2 \mathrm{~mL})$ following the typical procedure afforded $\mathbf{3 f}(94 \mathrm{mg}, 90 \%)$ after chromatography on silica gel (petroleum ether/ethyl acetate $=6: 1$ ) as a pale yellow solid; m.p. $211-213{ }^{\circ} \mathrm{C}$.

${ }^{1} \mathbf{H}$ NMR $\left(400 \mathrm{MHz}, \mathrm{CDCl}_{3}\right) \delta 11.18(\mathrm{~s}, 1 \mathrm{H}), 8.62(\mathrm{~d}, J=4.7 \mathrm{~Hz}, 2 \mathrm{H}), 8.18(\mathrm{~d}, J=8.3 \mathrm{~Hz}, 1 \mathrm{H})$, $7.44(\mathrm{~d}, J=7.7 \mathrm{~Hz}, 1 \mathrm{H}), 7.21$ (ddd, $J=8.4,7.0,1.3 \mathrm{~Hz}, 1 \mathrm{H}), 7.16-7.12(\mathrm{~m}, 1 \mathrm{H}), 7.08(\mathrm{t}, J=4.8 \mathrm{~Hz}$, $1 \mathrm{H}), 6.91-6.85(\mathrm{~m}, 2 \mathrm{H}), 6.74(\mathrm{~d}, J=8.7 \mathrm{~Hz}, 2 \mathrm{H}), 6.57(\mathrm{~d}, J=8.8 \mathrm{~Hz}, 2 \mathrm{H}), 6.45(\mathrm{~d}, J=8.8 \mathrm{~Hz}, 2 \mathrm{H})$, $6.43(\mathrm{~s}, 1 \mathrm{H}), 4.21-4.13(\mathrm{~m}, 2 \mathrm{H}), 3.64(\mathrm{~s}, 3 \mathrm{H}), 1.21(\mathrm{t}, J=7.1 \mathrm{~Hz}, 3 \mathrm{H})$.

${ }^{13} \mathbf{C}$ NMR $\left(101 \mathrm{MHz}, \mathrm{CDCl}_{3}\right) \delta 170.5,157.6,157.3,156.4,150.6,139.0,135.9,132.2,131.6,128.9$, 128.5, 128.3, 127.9, 124.3, 122.5, 122.1, 121.1, 117.0, 114.5, 112.5, 112.3, 101.9, 59.9, 55.0, 14.4.

IR (ATR): 2925, 1644, 1602, 1569, 1493, 1431, 1265, 1206, 1130, 1092, 910, 826, $751 \mathrm{~cm}^{-1}$.

MALDI-TOF-MS m/z calcd for $\mathrm{C}_{30} \mathrm{H}_{26} \mathrm{~N}_{4} \mathrm{O}_{3} \mathrm{Cl}[\mathrm{M}+\mathrm{H}]^{+}:$525.1688; found: 525.1690 .

Ethyl (Z)-2-phenyl-3-(1-(pyrimidin-2-yl)-1H-indol-2-yl)-3-((4-(trifluoromethyl)phenyl)amino) acrylate (3g)<smiles>CCOC(=O)/C(=C(\Nc1ccc(C(F)(F)F)cc1)c1cc2ccccc2n1-c1ncccn1)c1ccccc1</smiles>

The reaction of 1a $(0.2 \mathrm{mmol}), \mathbf{2 d}(0.25 \mathrm{mmol}),\left[\mathrm{Cp} * \mathrm{Co}(\mathrm{CO}) \mathrm{I}_{2}\right](0.005 \mathrm{mmol}), \operatorname{AgNTf}_{2}(0.015$ $\mathrm{mmol})$ in DCE $(2 \mathrm{~mL})$ following the typical procedure afforded $3 \mathrm{~g}(102 \mathrm{mg}, 97 \%)$ after chromatography on silica gel (petroleum ether/ethyl acetate $=6: 1$ ) as a pale yellow solid; m.p. $158-$ $160{ }^{\circ} \mathrm{C}$.

${ }^{1} \mathbf{H}$ NMR $\left(400 \mathrm{MHz}, \mathrm{CDCl}_{3}\right) \delta 11.42(\mathrm{~s}, 1 \mathrm{H}), 8.61(\mathrm{~d}, J=4.8 \mathrm{~Hz}, 2 \mathrm{H}), 8.18(\mathrm{~d}, J=8.4 \mathrm{~Hz}, 1 \mathrm{H})$, $7.45(\mathrm{~d}, J=7.7 \mathrm{~Hz}, 1 \mathrm{H}), 7.26-7.18(\mathrm{~m}, 3 \mathrm{H}), 7.17-7.13(\mathrm{~m}, 1 \mathrm{H}), 7.08(\mathrm{t}, J=4.8 \mathrm{~Hz}, 1 \mathrm{H}), 6.98-6.92$ 
$(\mathrm{m}, 1 \mathrm{H}), 6.88(\mathrm{t}, J=7.4 \mathrm{~Hz}, 2 \mathrm{H}), 6.80(\mathrm{~d}, J=8.5 \mathrm{~Hz}, 2 \mathrm{H}), 6.74-6.68(\mathrm{~m}, 2 \mathrm{H}), 6.51(\mathrm{~s}, 1 \mathrm{H}), 4.17(\mathrm{q}, J$ $=7.1 \mathrm{~Hz}, 2 \mathrm{H}), 1.19(\mathrm{t}, J=7.1 \mathrm{~Hz}, 3 \mathrm{H})$.

${ }^{13}$ C NMR $\left(101 \mathrm{MHz}, \mathrm{CDCl}_{3}\right) \delta 170.1,157.6,156.3,149.8,143.6,136.0,135.9,131.3,131.0,128.2$, $126.9,125.8,125.7(\mathrm{q}, J=3.9 \mathrm{~Hz}), 124.5,124.2(\mathrm{q}, J=269.7 \mathrm{~Hz}), 124.0(\mathrm{q}, J=32.6 \mathrm{~Hz}), 122.2,121.2$, $120.5,117.1,114.4,112.2,103.8,60.1,14.3$.

IR (ATR): 3053, 2983, 1646, 1602, 1571, 1533, 1429, 1320, 1256, 1207, 1163, 1115, 1067, 1046, $909,731,701 \mathrm{~cm}^{-1}$.

HRMS (EI-TOF) m/z calcd for $\mathrm{C}_{30} \mathrm{H}_{3} \mathrm{~N}_{4} \mathrm{O}_{3} \mathrm{~F}_{3}\left[\mathrm{M}^{+}\right]$: 528.1773; found: 528.1767.

Ethyl (Z)-2-phenyl-3-(1-(pyrimidin-2-yl)-1H-indol-2-yl)-3-(p-tolylamino)acrylate (3h)<smiles>CCOC(=O)/C(=C(\Nc1ccc(C)cc1)c1cc2ccccc2n1-c1ncccn1)c1ccccc1</smiles>

The reaction of $\mathbf{1 a}(0.2 \mathrm{mmol}), \mathbf{2 e}(0.25 \mathrm{mmol}),\left[\mathrm{Cp} * \mathrm{Co}(\mathrm{CO}) \mathrm{I}_{2}\right](0.005 \mathrm{mmol}), \mathrm{AgNTf}_{2}(0.015$ mmol) in DCE $(2 \mathrm{~mL})$ following the typical procedure afforded $\mathbf{3 h}(80 \mathrm{mg}, 84 \%)$ after chromatography on silica gel (petroleum ether/ethyl acetate $=6: 1$ ) as a pale yellow solid; m.p. $212-$ $214{ }^{\circ} \mathrm{C}$.

${ }^{1} \mathbf{H}$ NMR $\left(400 \mathrm{MHz}, \mathrm{CDCl}_{3}\right) \delta 11.25(\mathrm{~s}, 1 \mathrm{H}), 8.61(\mathrm{~d}, J=4.8 \mathrm{~Hz}, 2 \mathrm{H}), 8.16(\mathrm{~d}, J=8.3 \mathrm{~Hz}, 1 \mathrm{H})$, $7.42(\mathrm{~d}, J=7.4 \mathrm{~Hz}, 1 \mathrm{H}), 7.19$ (ddd, $J=8.4,7.1,1.4 \mathrm{~Hz}, 1 \mathrm{H}), 7.11(\mathrm{ddd}, J=8.1,7.1,1.1 \mathrm{~Hz}, 1 \mathrm{H}), 7.06$ $(\mathrm{t}, J=4.8 \mathrm{~Hz}, 1 \mathrm{H}), 6.95-6.90(\mathrm{~m}, 1 \mathrm{H}), 6.90-6.83(\mathrm{~m}, 2 \mathrm{H}), 6.79-6.74(\mathrm{~m}, 4 \mathrm{H}), 6.65-6.59(\mathrm{~m}, 2 \mathrm{H})$, $6.45(\mathrm{~d}, J=0.8 \mathrm{~Hz}, 1 \mathrm{H}), 4.20-4.13(\mathrm{~m}, 2 \mathrm{H}), 2.13(\mathrm{~s}, 3 \mathrm{H}), 1.19$ (t, $J=7.1 \mathrm{~Hz}, 3 \mathrm{H})$.

${ }^{13}$ C NMR $\left(101 \mathrm{MHz}, \mathrm{CDCl}_{3}\right) \delta 170.2,157.5,156.4,151.7,137.7,136.9,135.8,132.5,131.9,131.3$, 129.0, 128.4, 126.8, 125.4, 124.1, 121.9, 121.7, 121.0, 116.9, 114.3, 112.2, 100.9, 59.6, 20.7, 14.4.

IR (ATR): 2980, 1640, 1598, 1569, 1430, 1260, 1205, 1048, 908, 813, 729, $701 \mathrm{~cm}^{-1}$.

HRMS (EI-TOF) m/z calcd for $\mathrm{C}_{30} \mathrm{H}_{26} \mathrm{~N}_{4} \mathrm{O}_{2}\left[\mathrm{M}^{+}\right]$: 474.2056; found: 474.2064.

Ethyl (Z)-3-((4-methoxyphenyl)amino)-2-phenyl-3-(1-(pyrimidin-2-yl)-1H-indol-2-yl)acrylate (3i)<smiles>CCOC(=O)/C(=C(\Nc1ccc(OC)cc1)c1cc2ccccc2n1-c1ncccn1)c1ccccc1</smiles>

The reaction of $\mathbf{1 a}(0.2 \mathrm{mmol}), \mathbf{2 f}(0.25 \mathrm{mmol}),\left[\mathrm{Cp} * \mathrm{Co}(\mathrm{CO}) \mathrm{I}_{2}\right](0.005 \mathrm{mmol}), \operatorname{AgNTf}_{2}(0.015$ $\mathrm{mmol}$ ) in DCE ( $2 \mathrm{~mL}$ ) following the typical procedure afforded $3 \mathbf{i}(74 \mathrm{mg}, 77 \%)$ after chromatography on silica gel (petroleum ether/ethyl acetate $=6: 1$ ) as a pale yellow solid; m.p. $185-187^{\circ} \mathrm{C}$.

${ }^{1}$ H NMR $\left(400 \mathrm{MHz}, \mathrm{CDCl}_{3}\right) \delta 11.21(\mathrm{~s}, 1 \mathrm{H}), 8.62(\mathrm{~d}, J=4.7 \mathrm{~Hz}, 2 \mathrm{H}), 8.15(\mathrm{~d}, J=8.4 \mathrm{~Hz}, 1 \mathrm{H})$, $7.41(\mathrm{~d}, J=7.5 \mathrm{~Hz}, 1 \mathrm{H}), 7.18(\mathrm{ddd}, J=8.4,7.1,1.4 \mathrm{~Hz}, 1 \mathrm{H}), 7.13-7.05(\mathrm{~m}, 2 \mathrm{H}), 6.95-6.84(\mathrm{~m}, 3 \mathrm{H})$, $6.82-6.76(\mathrm{~m}, 2 \mathrm{H}), 6.71-6.66(\mathrm{~m}, 2 \mathrm{H}), 6.52-6.47(\mathrm{~m}, 2 \mathrm{H}), 6.43(\mathrm{~d}, J=0.8 \mathrm{~Hz}, 1 \mathrm{H}), 4.21-4.12(\mathrm{~m}$, 2H), 3.62 (s, 3H), 1.19 (t, $J=7.1 \mathrm{~Hz}, 3 \mathrm{H})$. 
${ }^{13} \mathbf{C}$ NMR $\left(101 \mathrm{MHz}, \mathrm{CDCl}_{3}\right) \delta 170.3,157.5,156.4,155.7,152.3,137.0,135.8,133.5,131.8,131.3$, 128.3, 126.8, 125.3, 124.1, 123.5, 121.9, 121.0, 116.9, 114.3, 113.7, 112.4, 100.3, 59.6, 55.2, 14.4.

IR (ATR): 2981, 1639, 1599, 1570, 1511, 1429, 1240, 1203, 1130, 1045, 909, 823, 794, 731, 701 $\mathrm{cm}^{-1}$.

HRMS (EI-TOF) m/z calcd for $\mathrm{C}_{30} \mathrm{H}_{26} \mathrm{~N}_{4} \mathrm{O}_{3}$ [M+]: 490.2005; found: 490.1999.

Ethyl (Z)-3-((3-chlorophenyl)amino)-2-phenyl-3-(1-(pyrimidin-2-yl)-1H-indol-2-yl)acrylate (3j)<smiles>CCOC(=O)/C(=C(\Nc1cccc(Cl)c1)c1cc2ccccc2n1-c1ncccn1)c1ccccc1</smiles>

The reaction of $\mathbf{1 a}(0.2 \mathrm{mmol}), \mathbf{2 g}(0.25 \mathrm{mmol}),\left[\mathrm{Cp} * \mathrm{Co}(\mathrm{CO}) \mathrm{I}_{2}\right](0.005 \mathrm{mmol}), \operatorname{AgNTf}_{2}(0.015$ $\mathrm{mmol}$ ) in DCE (2 mL) following the typical procedure afforded $\mathbf{3 j}$ ( $90 \mathrm{mg}, 91 \%)$ after chromatography on silica gel (petroleum ether/ethyl acetate $=6: 1$ ) as a pale yellow oil.

${ }^{1} \mathbf{H}$ NMR $\left(400 \mathrm{MHz}, \mathrm{CDCl}_{3}\right) \delta 11.20(\mathrm{~s}, 1 \mathrm{H}), 8.62(\mathrm{~d}, J=4.8 \mathrm{~Hz}, 2 \mathrm{H}), 8.15(\mathrm{~d}, J=8.4 \mathrm{~Hz}, 1 \mathrm{H})$, $7.43(\mathrm{~d}, J=7.7 \mathrm{~Hz}, 1 \mathrm{H}), 7.21(\mathrm{ddd}, J=8.5,7.0,1.4 \mathrm{~Hz}, 1 \mathrm{H}), 7.15-7.07(\mathrm{~m}, 2 \mathrm{H}), 6.97-6.88(\mathrm{~m}, 3 \mathrm{H})$, $6.87-6.82(\mathrm{~m}, 2 \mathrm{H}), 6.82-6.77(\mathrm{~m}, 2 \mathrm{H}), 6.71-6.69(\mathrm{~m}, 1 \mathrm{H}), 6.53-6.46(\mathrm{~m}, 1 \mathrm{H}), 6.46(\mathrm{~s}, 1 \mathrm{H}), 4.25-$ $4.11(\mathrm{~m}, 2 \mathrm{H}), 1.20(\mathrm{t}, J=7.1 \mathrm{~Hz}, 3 \mathrm{H})$.

${ }^{13}$ C NMR $\left(101 \mathrm{MHz}, \mathrm{CDCl}_{3}\right) \delta 170.2,157.6,156.4,150.6,141.6,136.6,135.9,133.9,131.4,131.3$, 129.4, 128.3, 126.9, 125.7, 124.4, 122.9, 122.1, 121.5, 121.1, 119.7, 117.0, 114.3, 112.7, 102.8, 59.9, 14.3.

IR (ATR): 2984, 1645, 1569, 1480, 1428, 1262, 1205, 1130, 1047, 750, $702 \mathrm{~cm}^{-1}$.

HRMS (EI-TOF) $\mathrm{m} / \mathrm{z}$ calcd for $\mathrm{C}_{29} \mathrm{H}_{23} \mathrm{~N}_{4} \mathrm{O}_{2} \mathrm{Cl}$ [M $\mathrm{M}^{+}$: 494.1510; found: 494.1512 .

Ethyl (Z)-3-((2-chlorophenyl)amino)-2-phenyl-3-(1-(pyrimidin-2-yl)-1H-indol-2-yl)acrylate (3k)<smiles>CCOC(=O)/C(=C(\Nc1ccccc1Cl)c1cc2ccccc2n1-c1ncccn1)c1ccccc1</smiles>

The reaction of 1a $(0.2 \mathrm{mmol}), \mathbf{2 h}(0.25 \mathrm{mmol}),\left[\mathrm{Cp} * \mathrm{Co}(\mathrm{CO}) \mathrm{I}_{2}\right](0.005 \mathrm{mmol}), \operatorname{AgNTf}_{2}(0.015$ $\mathrm{mmol})$ in DCE $(2 \mathrm{~mL})$ following the typical procedure afforded $\mathbf{3 k}(37 \mathrm{mg}, 37 \%)$ after chromatography on silica gel (petroleum ether/ethyl acetate $=6: 1$ ) as a pale yellow oil.

${ }^{1} \mathbf{H}$ NMR $\left(400 \mathrm{MHz}, \mathrm{CDCl}_{3}\right) \delta 11.31(\mathrm{~s}, 1 \mathrm{H}), 8.62(\mathrm{~d}, J=4.8 \mathrm{~Hz}, 2 \mathrm{H}), 8.14(\mathrm{~d}, J=8.3 \mathrm{~Hz}, 1 \mathrm{H})$, $7.43(\mathrm{~d}, J=7.7 \mathrm{~Hz}, 1 \mathrm{H}), 7.23-7.18(\mathrm{~m}, 1 \mathrm{H}), 7.17(\mathrm{dd}, J=7.9,1.5 \mathrm{~Hz}, 1 \mathrm{H}), 7.15-7.10(\mathrm{~m}, 1 \mathrm{H}), 7.08$ $(\mathrm{t}, J=4.8 \mathrm{~Hz}, 1 \mathrm{H}), 7.01-6.89(\mathrm{~m}, 5 \mathrm{H}), 6.75-6.70(\mathrm{~m}, 1 \mathrm{H}), 6.69-6.65(\mathrm{~m}, 1 \mathrm{H}), 6.55(\mathrm{dd}, J=8.1,1.6$ $\mathrm{Hz}, 1 \mathrm{H}), 6.47$ (s, 1H), $4.30-4.14(\mathrm{~m}, 2 \mathrm{H}), 1.22(\mathrm{t}, J=7.1 \mathrm{~Hz}, 3 \mathrm{H})$.

${ }^{13} \mathbf{C}$ NMR $\left(101 \mathrm{MHz}, \mathrm{CDCl}_{3}\right) \delta 170.0,157.7,156.3,149.5,137.3,136.8,135.9,131.6,131.4,128.8$, 128.3, 126.9, 126.8, 125.7, 124.9, 124.3, 123.1, 122.4, 122.1, 121.1, 117.0, 114.3, 112.7, 103.8, 60.0, 14.4 . 
IR (ATR): 2984, 1648, 1591, 1567, 1485, 1427, 1310, 1262, 1204, 1131, 1047, 910, 796, 750, 701 $\mathrm{cm}^{-1}$.

HRMS (EI-TOF) m/z calcd for $\mathrm{C}_{29} \mathrm{H}_{23} \mathrm{~N}_{4} \mathrm{O}_{2} \mathrm{Cl}\left[\mathrm{M}^{+}\right]$: 494.1510 ; found: 494.1507.

Ethyl (Z)-3-(ethylamino)-2-phenyl-3-(1-(pyrimidin-2-yl)-1H-indol-2-yl)acrylate (3l)<smiles>CCNC(=C(C(=O)OCC)c1cc2ccccc2n1-c1ncccn1)c1ccccc1</smiles>

The reaction of $\mathbf{1 a}(0.2 \mathrm{mmol}), \mathbf{2} \mathbf{i}(0.25 \mathrm{mmol}),\left[\mathrm{Cp} * \mathrm{Co}(\mathrm{CO}) \mathrm{I}_{2}\right](0.005 \mathrm{mmol}), \operatorname{AgNTf}_{2}(0.015$ $\mathrm{mmol})$ in DCE $(2 \mathrm{~mL})$ following the typical procedure afforded 31 ( $80 \mathrm{mg}, 97 \%)$ after chromatography on silica gel (petroleum ether/ethyl acetate $=6: 1$ ) as a pale yellow solid; m.p. $102-103{ }^{\circ} \mathrm{C}$.

${ }^{1} \mathbf{H}$ NMR $\left(400 \mathrm{MHz}, \mathrm{CDCl}_{3}\right) \delta 9.36(\mathrm{t}, J=5.1 \mathrm{~Hz}, 1 \mathrm{H}), 8.70(\mathrm{~d}, J=4.8 \mathrm{~Hz}, 2 \mathrm{H}), 8.27(\mathrm{~d}, J=8.4 \mathrm{~Hz}$, $1 \mathrm{H}), 7.50(\mathrm{~d}, J=7.7 \mathrm{~Hz}, 1 \mathrm{H}), 7.25-7.19(\mathrm{~m}, 1 \mathrm{H}), 7.17-7.15(\mathrm{~m}, 1 \mathrm{H}), 7.11(\mathrm{t}, J=4.8 \mathrm{~Hz}, 1 \mathrm{H}), 6.89-$ $6.80(\mathrm{~m}, 3 \mathrm{H}), 6.76-6.67(\mathrm{~m}, 2 \mathrm{H}), 6.53(\mathrm{~s}, 1 \mathrm{H}), 4.20-4.03(\mathrm{~m}, 2 \mathrm{H}), 3.19-3.01(\mathrm{~m}, 2 \mathrm{H}), 1.18-1.10$ $(\mathrm{m}, 6 \mathrm{H})$.

${ }^{13}$ C NMR $\left(101 \mathrm{MHz}, \mathrm{CDCl}_{3}\right) \delta 170.3,157.7,157.3,156.8,137.7,135.7,132.1,131.1,128.5,126.8$, 125.0, 124.0, 122.1, 120.8, 117.0, 114.5, 110.5, 97.6, 59.0, 40.0, 15.8, 14.5.

IR (ATR): 3255, 2977, 1636, 1571, 1426, 1314, 1256, 1208, 1138, 1093, 1048, 910, 795, 731, 702 $\mathrm{cm}^{-1}$.

HRMS (EI-TOF) m/z calcd for $\mathrm{C}_{25} \mathrm{H}_{24} \mathrm{~N}_{4} \mathrm{O}_{2}\left[\mathrm{M}^{+}\right]$: 412.1899; found: 412.1902 .

Ethyl (Z)-3-(isopropylamino)-2-phenyl-3-(1-(pyrimidin-2-yl)-1H-indol-2-yl)acrylate (3m)<smiles>CCOC(=O)/C(=C(\NC(C)C)c1cc2ccccc2n1-c1ncccn1)c1ccccc1</smiles>

The reaction of $\mathbf{1 a}(0.2 \mathrm{mmol}), \mathbf{2} \mathbf{j}(0.25 \mathrm{mmol}),\left[\mathrm{Cp} * \mathrm{Co}(\mathrm{CO}) \mathrm{I}_{2}\right](0.005 \mathrm{mmol}), \operatorname{AgNTf}_{2}(0.015$ $\mathrm{mmol})$ in DCE $(2 \mathrm{~mL})$ following the typical procedure afforded $\mathbf{3 m}(74 \mathrm{mg}, 87 \%)$ after chromatography on silica gel (petroleum ether/ethyl acetate $=6: 1$ ) as a pale yellow solid; m.p. $123-$ $126{ }^{\circ} \mathrm{C}$.

${ }^{1} \mathbf{H}$ NMR $\left(400 \mathrm{MHz}, \mathrm{CDCl}_{3}\right) \delta 9.37(\mathrm{~d}, J=9.6 \mathrm{~Hz}, 1 \mathrm{H}), 8.69(\mathrm{~d}, J=4.8 \mathrm{~Hz}, 2 \mathrm{H}), 8.23(\mathrm{~d}, J=8.4$ $\mathrm{Hz}, 1 \mathrm{H}), 7.54-7.49(\mathrm{~m}, 1 \mathrm{H}), 7.22(\mathrm{ddd}, J=8.4,7.1,1.4 \mathrm{~Hz}, 1 \mathrm{H}), 7.18-7.14(\mathrm{~m}, 1 \mathrm{H}), 7.11(\mathrm{t}, J=4.8$ $\mathrm{Hz}, 1 \mathrm{H}), 6.88-6.78(\mathrm{~m}, 3 \mathrm{H}), 6.71-6.65(\mathrm{~m}, 2 \mathrm{H}), 6.58(\mathrm{~d}, J=0.8 \mathrm{~Hz}, 1 \mathrm{H}), 4.18-4.02(\mathrm{~m}, 2 \mathrm{H})$, 3.56-3.47 (m, 1H), $1.21-1.06(\mathrm{~m}, 9 \mathrm{H})$.

${ }^{13}$ C NMR $\left(101 \mathrm{MHz}, \mathrm{CDCl}_{3}\right) \delta 170.2,157.7,156.7,156.3,137.6,135.7,132.2,131.1,128.4,126.7$, 125.0, 123.9, 122.0, 120.8, 117.0, 114.3, 110.5, 97.2, 58.9, 47.1, 24.9, 23.7, 14.5.

IR (ATR): 3248, 2974, 1638, 1574, 1429, 1313, 1260, 1217, 1154, 1130, 1049, 911, 798, 748, 703 $\mathrm{cm}^{-1}$.

HRMS (EI-TOF) m/z calcd for $\mathrm{C}_{26} \mathrm{H}_{26} \mathrm{~N}_{4} \mathrm{O}_{2}\left[\mathrm{M}^{+}\right]$: 426.2056 ; found: 426.2060 . 
Ethyl (Z)-3-(cyclohexylamino)-2-phenyl-3-(1-(pyrimidin-2-yl)-1H-indol-2-yl)acrylate (3n)<smiles>CCOC(=O)/C(=C(\NC1CCCCC1)c1cc2ccccc2n1-c1ncccn1)c1ccccc1</smiles>

The reaction of $\mathbf{1 a}(0.2 \mathrm{mmol}), \mathbf{2 k}(0.25 \mathrm{mmol}),\left[\mathrm{Cp} * \mathrm{Co}(\mathrm{CO}) \mathrm{I}_{2}\right](0.005 \mathrm{mmol}), \operatorname{AgNTf}_{2}(0.015$ mmol) in DCE $(2 \mathrm{~mL})$ following the typical procedure afforded $3 \mathbf{n}(86 \mathrm{mg}, 92 \%)$ after chromatography on silica gel (petroleum ether/ethyl acetate $=6: 1$ ) as a pale yellow solid; m.p. 193 $194{ }^{\circ} \mathrm{C}$.

${ }^{1} \mathbf{H}$ NMR $\left(400 \mathrm{MHz}, \mathrm{CDCl}_{3}\right) \delta 9.57(\mathrm{~d}, J=9.5 \mathrm{~Hz}, 1 \mathrm{H}), 8.67(\mathrm{~d}, J=4.8 \mathrm{~Hz}, 2 \mathrm{H}), 8.21(\mathrm{~d}, J=8.3$ $\mathrm{Hz}, 1 \mathrm{H}), 7.54$ (d, $J=7.2 \mathrm{~Hz}, 1 \mathrm{H}), 7.22$ (ddd, $J=8.4,7.1,1.5 \mathrm{~Hz}, 1 \mathrm{H}), 7.19-7.15(\mathrm{~m}, 1 \mathrm{H}), 7.10(\mathrm{t}, J=$ $4.8 \mathrm{~Hz}, 1 \mathrm{H}), 6.86-6.76(\mathrm{~m}, 3 \mathrm{H}), 6.60(\mathrm{t}, J=3.3 \mathrm{~Hz}, 3 \mathrm{H}), 4.15-4.04(\mathrm{~m}, 2 \mathrm{H}), 3.31-3.18(\mathrm{~m}, 1 \mathrm{H})$, $1.95-1.84(\mathrm{~m}, 1 \mathrm{H}), 1.82-1.73(\mathrm{~m}, 1 \mathrm{H}), 1.73-1.59(\mathrm{~m}, 2 \mathrm{H}), 1.47-1.11(\mathrm{~m}, 9 \mathrm{H})$.

${ }^{13}$ C NMR $\left(101 \mathrm{MHz} \mathrm{CDCl}_{3}\right) \delta 170.2,157.6,156.6,156.3,137.6,135.7,132.2,131.0,128.4,126.7$, 124.9, 123.9, 122.0, 120.9, 117.0, 114.3, 110.4, 97.0, 58.9, 53.7, 35.1, 33.9, 25.4, 24.6, 24.5, 14.5.

IR (ATR): 3247, 2930, 2853, 1634, 1572, 1427, 1313, 1256, 1203, 1132, 1098, 1049, 909, 797, 730, $702 \mathrm{~cm}^{-1}$.

HRMS (EI-TOF) m/z calcd for $\mathrm{C}_{29} \mathrm{H}_{30} \mathrm{~N}_{4} \mathrm{O}_{2}\left[\mathrm{M}^{+}\right]$: 466.2369; found: 466.2372.

Ethyl (Z)-3-((4-chlorophenyl)amino)-2-phenyl-3-(1-(pyrimidin-2-yl)indolin-7-yl)acrylate (3o)<smiles>CCOC(=O)/C(=C(\Nc1ccc(Cl)cc1)c1cccc2c1N(c1ncccn1)CC2)c1ccccc1</smiles>

The reaction of 1e $(0.2 \mathrm{mmol}), \mathbf{2 a}(0.25 \mathrm{mmol}),\left[\mathrm{Cp} * \mathrm{Co}(\mathrm{CO}) \mathrm{I}_{2}\right](0.005 \mathrm{mmol}), \operatorname{AgNTf}_{2}(0.015$ $\mathrm{mmol})$ in DCE (2 mL) following the typical procedure afforded $3 \mathbf{3}(37 \mathrm{mg}, 37 \%)$ after chromatography on silica gel (petroleum ether/ethyl acetate $=6: 1$ ) as a pale yellow solid; m.p. $178-181{ }^{\circ} \mathrm{C}$.

${ }^{1}$ H NMR $\left(400 \mathrm{MHz}, \mathrm{CDCl}_{3}\right) \delta 11.31(\mathrm{~s}, 1 \mathrm{H}), 8.27(\mathrm{~d}, J=4.7 \mathrm{~Hz}, 2 \mathrm{H}), 7.06-6.96(\mathrm{~m}, 8 \mathrm{H}), 6.88(\mathrm{~d}$, $J=7.7 \mathrm{~Hz}, 1 \mathrm{H}), 6.75(\mathrm{t}, J=7.5 \mathrm{~Hz}, 1 \mathrm{H}), 6.65-6.58(\mathrm{~m}, 3 \mathrm{H}), 4.15-3.95(\mathrm{~m}, 2 \mathrm{H}), 3.94-3.88(\mathrm{~m}, 1 \mathrm{H})$, $3.12(\mathrm{q}, J=9.6 \mathrm{~Hz}, 1 \mathrm{H}), 2.97-2.89(\mathrm{~m}, 1 \mathrm{H}), 2.63-2.55(\mathrm{~m}, 1 \mathrm{H}), 1.09(\mathrm{t}, J=7.1 \mathrm{~Hz}, 3 \mathrm{H})$.

${ }^{13} \mathbf{C}$ NMR $\left(101 \mathrm{MHz}, \mathrm{CDCl}_{3}\right) \delta 169.8,158.4,157.7,156.3,141.2,139.2,136.8,134.5,132.3,129.1$, $128.2,127.2,126.2,125.0,124.9,122.9,121.8,121.7,112.2,100.2,59.4,49.7,27.9,14.3$.

IR (ATR): 2983, 1640, 1566, 1460, 1385, 1257, 1205, 1149, 1093, 1039, 909, 823, 792, 747, 702 $\mathrm{cm}^{-1}$.

HRMS (EI-TOF) m/z calcd for $\mathrm{C}_{29} \mathrm{H}_{25} \mathrm{~N}_{4} \mathrm{O}_{2} \mathrm{Cl}\left[\mathrm{M}^{+}\right]$: 496.1666; found: 496.1660 .

Ethyl (Z)-3-((4-chlorophenyl)amino)-2-phenyl-3-(1-(pyrimidin-2-yl)-1H-pyrrol-2-yl)acrylate (3p) 


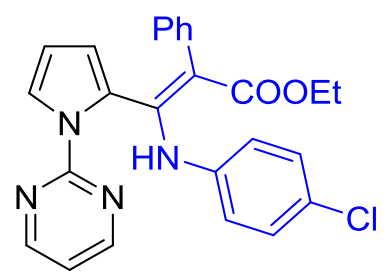

The reaction of $\mathbf{1 f}(0.2 \mathrm{mmol}), \mathbf{2 a}(0.25 \mathrm{mmol}),\left[\mathrm{Cp} * \mathrm{Co}(\mathrm{CO}) \mathrm{I}_{2}\right](0.005 \mathrm{mmol}), \operatorname{AgNTf}_{2}(0.015$ $\mathrm{mmol})$ in DCE $(2 \mathrm{~mL})$ following the typical procedure afforded $\mathbf{3 p}(65 \mathrm{mg}, 73 \%)$ after chromatography on silica gel (petroleum ether/ethyl acetate $=6: 1$ ) as a pale yellow solid; m.p. $161-163{ }^{\circ} \mathrm{C}$.

${ }^{1}$ H NMR $\left(400 \mathrm{MHz}, \mathrm{CDCl}_{3}\right) \delta 11.15(\mathrm{~s}, 1 \mathrm{H}), 8.52(\mathrm{~d}, J=4.8 \mathrm{~Hz}, 2 \mathrm{H}), 7.33(\mathrm{dd}, J=3.1,1.8 \mathrm{~Hz}$, 1H), $7.05(\mathrm{t}, J=4.8 \mathrm{~Hz}, 1 \mathrm{H}), 7.02-6.94(\mathrm{~m}, 5 \mathrm{H}), 6.81-6.79(\mathrm{~m}, 2 \mathrm{H}), 6.59-6.53(\mathrm{~m}, 2 \mathrm{H}), 6.09(\mathrm{t}, J=$ $3.3 \mathrm{~Hz}, 1 \mathrm{H}), 6.02(\mathrm{dd}, J=3.5,1.7 \mathrm{~Hz}, 1 \mathrm{H}), 4.21-4.12(\mathrm{~m}, 2 \mathrm{H}), 1.20(\mathrm{t}, J=7.1 \mathrm{~Hz}, 3 \mathrm{H})$.

${ }^{13}$ C NMR $\left(101 \mathrm{MHz}, \mathrm{CDCl}_{3}\right) \delta 170.3,157.9,155.6,151.3,139.2,137.2,131.4,128.4,127.8,126.7$, $125.25,125.23,122.6,121.9,117.8,117.6,110.8,102.0,59.7,14.4$.

IR (ATR): 2983, 1643, 1594, 1568, 1492, 1440, 1258, 1214, 1194, 1103, 1039, 1009, 911, 748, 701 $\mathrm{cm}^{-1}$.

HRMS (EI-TOF) m/z calcd for $\mathrm{C}_{25} \mathrm{H}_{21} \mathrm{~N}_{4} \mathrm{O}_{2} \mathrm{Cl}\left[\mathrm{M}^{+}\right]$: 444.1353 ; found: 444.1352 .

\section{Ethyl (Z)-2-phenyl-3-(1-(pyrimidin-2-yl)-1H-pyrrol-2-yl)-3-((4-(trifluoromethyl)phenyl)amino)} acrylate (3q)

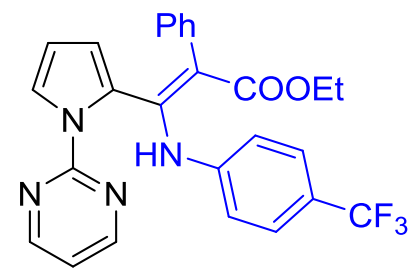

The reaction of $\mathbf{1 f}(0.2 \mathrm{mmol}), \mathbf{2 d}(0.25 \mathrm{mmol}),\left[\mathrm{Cp} * \mathrm{Co}(\mathrm{CO}) \mathrm{I}_{2}\right](0.005 \mathrm{mmol}), \operatorname{AgNTf}_{2}(0.015$ $\mathrm{mmol})$ in DCE ( $2 \mathrm{~mL}$ ) following the typical procedure afforded $\mathbf{3 q}(75 \mathrm{mg}, 78 \%)$ after chromatography on silica gel (petroleum ether/ethyl acetate $=6: 1$ ) as a pale yellow solid; m.p. $153-154{ }^{\circ} \mathrm{C}$.

${ }^{1}$ H NMR (400 MHz, Chloroform- $d$ ) $\delta 11.29$ (s, 1H), 8.51 (d, $\left.J=4.8 \mathrm{~Hz}, 2 \mathrm{H}\right), 7.38$ (dd, $J=3.1,1.8$ $\mathrm{Hz}, 1 \mathrm{H}), 7.25$ (d, $J=8.4 \mathrm{~Hz}, 2 \mathrm{H}), 7.04(\mathrm{t}, J=4.8 \mathrm{~Hz}, 1 \mathrm{H}), 7.03-6.95(\mathrm{~m}, 3 \mathrm{H}), 6.83-6.76(\mathrm{~m}, 2 \mathrm{H})$, $6.65(\mathrm{~d}, J=8.5 \mathrm{~Hz}, 2 \mathrm{H}), 6.11(\mathrm{t}, J=3.3 \mathrm{~Hz}, 1 \mathrm{H}), 6.06(\mathrm{dd}, J=3.5,1.8 \mathrm{~Hz}, 1 \mathrm{H}), 4.18(\mathrm{br}, 2 \mathrm{H}), 1.20(\mathrm{t}, J$ $=7.1 \mathrm{~Hz}, 3 \mathrm{H})$.

${ }^{13}$ C NMR (101 MHz, Chloroform- $d$ ) $\delta$ 170.2, 157.9, 155.6, 150.2, 143.8, 136.9, 131.3, 126.8, 125.7 $(\mathrm{q}, J=3.8 \mathrm{~Hz}), 125.5,125.1,123.8(\mathrm{q}, J=32.5 \mathrm{~Hz}), 122.1,120.2,117.75,117.66,111.0,103.6,60.0$, 14.3 .

HRMS (EI-TOF) m/z calcd for $\mathrm{C}_{26} \mathrm{H}_{21} \mathrm{~N}_{4} \mathrm{O}_{2} \mathrm{~F}_{3}\left[\mathrm{M}^{+}\right]$: 478.1617; found: 478.1618.

Typical procedure for the preparation of Ethyl (Z)-3-((4-chlorophenyl)amino)-2-phenyl-3-(2(pyrimidin-2-yl)phenyl)acrylate (5a) 

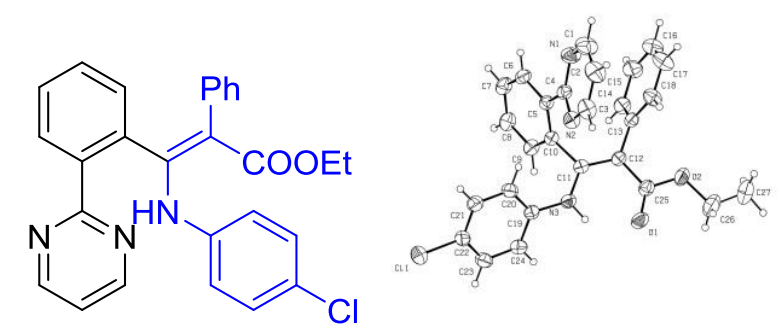

To an oven-dried Schlenk tube equipped with a magnetic stirring bar were added sequentially $4 \mathbf{a}$ $(0.2 \mathrm{mmol}), 2 \mathbf{a}(0.25 \mathrm{mmol}),\left[\mathrm{Cp} * \mathrm{Co}(\mathrm{CO}) \mathrm{I}_{2}\right](0.005 \mathrm{mmol}), \operatorname{AgNTf}_{2}(0.015 \mathrm{mmol})$ and $\operatorname{dry} \mathrm{DCE}(2 \mathrm{~mL})$ under $\mathrm{N}_{2}$ atmosphere. The reaction vessel was heated to $80{ }^{\circ} \mathrm{C}$ in oil bath for 10 hours. Upon completion, the reaction mixture was cooled to room temperature and then the solvent was evaporated in vacuum. The residue was purified by column chromatography on silica gel (petroleum ether/ethyl acetate $=6: 1)$ to give the product $\mathbf{5 a}(36 \mathrm{mg}, 40 \%)$ as a pale yellow solid; m.p. $183-185{ }^{\circ} \mathrm{C}$.

${ }^{1} \mathbf{H}$ NMR $\left(400 \mathrm{MHz}, \mathrm{CDCl}_{3}\right) \delta 11.49(\mathrm{~s}, 1 \mathrm{H}), 8.70(\mathrm{~d}, J=4.8 \mathrm{~Hz}, 2 \mathrm{H}), 7.78(\mathrm{dd}, J=7.6,1.4 \mathrm{~Hz}$, 1H), 7.29-7.25 (m, 1H), 7.25-7.21 (m, 1H), 7.20-7.17 (m, 1H), 7.14 (dd, J = 7.5, $1.4 \mathrm{~Hz}, 1 \mathrm{H}), 6.97-$ $6.89(\mathrm{~m}, 3 \mathrm{H}), 6.89-6.81(\mathrm{~m}, 2 \mathrm{H}), 6.70-6.63(\mathrm{~m}, 2 \mathrm{H}), 6.57-6.49(\mathrm{~m}, 2 \mathrm{H}), 4.13(\mathrm{q}, J=7.1 \mathrm{~Hz}, 2 \mathrm{H})$, $1.16(\mathrm{t}, J=7.1 \mathrm{~Hz}, 3 \mathrm{H})$.

${ }^{13}$ C NMR $\left(101 \mathrm{MHz}, \mathrm{CDCl}_{3}\right) \delta 170.3,164.8,159.1,156.6,138.9,138.0,136.6,134.3,131.9,131.2$, 130.0, 129.3, 128.7, 128.3, 128.0, 126.6, 125.3, 123.4, 118.8, 100.7, 59.6, 14.4.

IR (ATR): 2926, 1642, 1589, 1563, 1494, 1415, 1261, 1210, 1141, 1093, 1034, 910, 754, $703 \mathrm{~cm}^{-1}$.

HRMS (EI-TOF) m/z calcd for $\mathrm{C}_{27} \mathrm{H}_{22} \mathrm{~N}_{3} \mathrm{O}_{2} \mathrm{Cl}\left[\mathrm{M}^{+}\right]$: 455.1401 ; found: 455.1403 .

Ethyl (Z)-3-((4-chlorophenyl)amino)-3-(5-methoxy-2-(pyrimidin-2-yl)phenyl)-2-phenylacrylate (5b)<smiles>CCOC(=O)/C(=C(\Nc1ccc(Cl)cc1)c1cc(OC)ccc1-c1ncccn1)c1ccccc1</smiles>

The reaction of $\mathbf{4 b}(0.2 \mathrm{mmol}), \mathbf{2 a}(0.25 \mathrm{mmol})$, [Cp*Co(CO) $\left.\mathrm{I}_{2}\right](0.005 \mathrm{mmol}), \operatorname{AgNTf}_{2}(0.015$ mmol) in DCE (2 $\mathrm{mL})$ following the typical procedure afforded $\mathbf{5 b}(43 \mathrm{mg}, 44 \%)$ after chromatography on silica gel (petroleum ether/ethyl acetate $=6: 1$ ) as a pale yellow solid; m.p. $183-$ $185^{\circ} \mathrm{C}$.

${ }^{1} \mathbf{H}$ NMR $\left(400 \mathrm{MHz}, \mathrm{CDCl}_{3}\right) \delta 11.44(\mathrm{~s}, 1 \mathrm{H}), 8.65(\mathrm{~d}, J=4.8 \mathrm{~Hz}, 2 \mathrm{H}), 7.75(\mathrm{~d}, J=8.7 \mathrm{~Hz}, 1 \mathrm{H})$, $7.11(\mathrm{t}, J=4.8 \mathrm{~Hz}, 1 \mathrm{H}), 6.98-6.85(\mathrm{~m}, 5 \mathrm{H}), 6.78(\mathrm{dd}, J=8.7,2.6 \mathrm{~Hz}, 1 \mathrm{H}), 6.67-6.64(\mathrm{~m}, 3 \mathrm{H}), 6.61$ $(\mathrm{d}, J=7.1 \mathrm{~Hz}, 2 \mathrm{H}), 4.20-4.08(\mathrm{~m}, 2 \mathrm{H}), 3.66(\mathrm{~s}, 3 \mathrm{H}), 1.16(\mathrm{t}, J=7.1 \mathrm{~Hz}, 3 \mathrm{H})$.

${ }^{13}$ C NMR $\left(101 \mathrm{MHz}, \mathrm{CDCl}_{3}\right) \delta 170.4,164.4,160.3,159.0,156.5,138.8,136.7,135.7,131.8,131.6$, 130.9, 128.3, 128.1, 126.7, 125.4, 123.3, 118.2, 116.2, 114.6, 100.5, 59.6, 55.4, 14.4.

IR (ATR): 2984, 1643, 1589, 1564, 1493, 1414, 1263, 1210, 1094, 1032, 911, 799, 750, $703 \mathrm{~cm}^{-1}$.

HRMS (EI-TOF) m/z calcd for $\mathrm{C}_{28} \mathrm{H}_{24} \mathrm{~N}_{3} \mathrm{O}_{3} \mathrm{Cl}\left[\mathrm{M}^{+}\right]$: 485.1506 ; found: 485.1505 .

Ethyl (Z)-3-(5-acetyl-2-(pyrimidin-2-yl)phenyl)-3-((4-chlorophenyl)amino)-2-phenylacrylate (5c) 
<smiles>CCOC(=O)/C(=C(\Nc1ccc(Cl)cc1)c1cc(C(C)=O)ccc1-c1ncccn1)c1ccccc1</smiles>

The reaction of $\mathbf{4 c}(0.2 \mathrm{mmol}), \mathbf{2 a}(0.25 \mathrm{mmol}),\left[\mathrm{Cp} * \mathrm{Co}(\mathrm{CO}) \mathrm{I}_{2}\right](0.005 \mathrm{mmol}), \mathrm{AgNTf}_{2}(0.015$ $\mathrm{mmol})$ in DCE $(2 \mathrm{~mL})$ following the typical procedure afforded $\mathbf{5 c}(37 \mathrm{mg}, 37 \%)$ after chromatography on silica gel (petroleum ether/ethyl acetate $=6: 1$ ) as a pale yellow solid; m.p. $172-174{ }^{\circ} \mathrm{C}$.

${ }^{1} \mathbf{H}$ NMR $\left(400 \mathrm{MHz}, \mathrm{CDCl}_{3}\right) \delta 11.38(\mathrm{~s}, 1 \mathrm{H}), 8.73(\mathrm{~d}, J=4.8 \mathrm{~Hz}, 2 \mathrm{H}), 7.88(\mathrm{~d}, J=8.2 \mathrm{~Hz}, 1 \mathrm{H})$, $7.82(\mathrm{dd}, J=8.2,1.8 \mathrm{~Hz}, 1 \mathrm{H}), 7.71(\mathrm{~d}, J=1.7 \mathrm{~Hz}, 1 \mathrm{H}), 7.23(\mathrm{t}, J=4.8 \mathrm{~Hz}, 1 \mathrm{H}), 6.97-6.84(\mathrm{~m}, 5 \mathrm{H})$, $6.66-6.61(\mathrm{~m}, 2 \mathrm{H}), 6.61-6.56(\mathrm{~m}, 2 \mathrm{H}), 4.24-4.07(\mathrm{~m}, 2 \mathrm{H}), 2.45(\mathrm{~s}, 3 \mathrm{H}), 1.18(\mathrm{~s}, 3 \mathrm{H})$.

${ }^{13} \mathbf{C}$ NMR $\left(101 \mathrm{MHz}, \mathrm{CDCl}_{3}\right) \delta 197.0,170.3,163.9,158.0,156.8,141.9,138.5,137.0,136.5,134.6$, 132.0, 131.9, 130.5, 128.5, 128.0, 126.9, 125.5, 123.7, 119.3, 101.2, 59.7, 26.7, 14.3.

IR (ATR): 2984, 1688, 1644, 1588, 1564, 1494, 1417, 1273, 1211, 1093, 1039, 912, 815, 751, 703 $\mathrm{cm}^{-1}$.

HRMS (EI-TOF) m/z calcd for $\mathrm{C}_{29} \mathrm{H}_{24} \mathrm{~N}_{3} \mathrm{O}_{3} \mathrm{Cl}\left[\mathrm{M}^{+}\right]$: 497.1506; found: 497.1503 .

Ethyl (Z)-3-((4-chlorophenyl)amino)-3-(4-methyl-2-(pyrimidin-2-yl)phenyl)-2-phenylacrylate (5d)<smiles>CCOC(=O)/C(=C(\Nc1ccc(Cl)cc1)c1ccc(C)cc1-c1ncccn1)c1ccccc1</smiles>

The reaction of $\mathbf{4 d}(0.2 \mathrm{mmol}), \mathbf{2 a}(0.25 \mathrm{mmol}),\left[\mathrm{Cp} * \mathrm{Co}(\mathrm{CO}) \mathrm{I}_{2}\right](0.005 \mathrm{mmol}), \operatorname{AgNTf}_{2}(0.015$ $\mathrm{mmol})$ in DCE (2 $\mathrm{mL})$ following the typical procedure afforded $\mathbf{5 d}(38 \mathrm{mg}, 41 \%)$ after chromatography on silica gel (petroleum ether/ethyl acetate $=6: 1$ ) as a pale yellow solid; m.p. $161-163{ }^{\circ} \mathrm{C}$.

${ }^{1} \mathbf{H}$ NMR $\left(400 \mathrm{MHz}, \mathrm{CDCl}_{3}\right) \delta 11.47(\mathrm{~s}, 1 \mathrm{H}), 8.69(\mathrm{~d}, J=4.8 \mathrm{~Hz}, 2 \mathrm{H}), 7.59(\mathrm{~s}, 1 \mathrm{H}), 7.16(\mathrm{t}, J=4.9$ $\mathrm{Hz}, 1 \mathrm{H}), 7.03(\mathrm{~s}, 2 \mathrm{H}), 6.98-6.90(\mathrm{~m}, 3 \mathrm{H}), 6.86(\mathrm{t}, J=7.4 \mathrm{~Hz}, 2 \mathrm{H}), 6.70-6.64(\mathrm{~m}, 2 \mathrm{H}), 6.53(\mathrm{~d}, J=$ $7.1 \mathrm{~Hz}, 2 \mathrm{H}), 4.13(\mathrm{q}, J=7.1 \mathrm{~Hz}, 2 \mathrm{H}), 2.29(\mathrm{~s}, 3 \mathrm{H}), 1.15(\mathrm{t}, J=7.1 \mathrm{~Hz}, 3 \mathrm{H})$.

${ }^{13} \mathbf{C}$ NMR $\left(101 \mathrm{MHz}, \mathrm{CDCl}_{3}\right) \delta 170.4,165.0,159.2,156.6,139.0,138.7,137.9,136.7,132.0,131.4$, $131.1,130.5,130.2,128.3,127.9,126.6,125.2,123.4,118.7,100.8,59.5,21.1,14.4$.

IR (ATR): 2983, 1641, 1589, 1562, 1493, 1422, 1395, 1261, 1208, 1129, 1091, 1033, 821, 793, 745, $701 \mathrm{~cm}^{-1}$.

HRMS (EI-TOF) m/z calcd for $\mathrm{C}_{28} \mathrm{H}_{24} \mathrm{~N}_{3} \mathrm{O}_{2} \mathrm{Cl}$ [M+ $\mathrm{M}^{+}$: 469.1557; found: 469.1555 .

Ethyl (Z)-3-((4-chlorophenyl)amino)-3-(3-methyl-2-(pyrimidin-2-yl)phenyl)-2-phenylacrylate (5e)<smiles>CCOC(=O)C(=C(Nc1ccc(Cl)cc1)c1cccc(C)c1-c1ncccn1)c1ccccc1</smiles> 
The reaction of $4 \mathbf{e}(0.2 \mathrm{mmol}), \mathbf{2 a}(0.25 \mathrm{mmol}),\left[\mathrm{Cp} * \mathrm{Co}(\mathrm{CO}) \mathrm{I}_{2}\right](0.005 \mathrm{mmol}), \mathrm{AgNTf}_{2}(0.015$ $\mathrm{mmol})$ in DCE (2 mL) following the typical procedure afforded $5 \mathbf{e}(30 \mathrm{mg}, 32 \%)$ after chromatography on silica gel (petroleum ether/ethyl acetate $=6: 1$ ) as a pale yellow solid; m.p. $212-214{ }^{\circ} \mathrm{C}$.

${ }^{1}$ H NMR $\left(400 \mathrm{MHz}, \mathrm{CDCl}_{3}\right) \delta 11.11(\mathrm{~s}, 1 \mathrm{H}), 8.66(\mathrm{~d}, J=4.9 \mathrm{~Hz}, 2 \mathrm{H}), 7.15(\mathrm{t}, J=4.9 \mathrm{~Hz}, 1 \mathrm{H}), 7.10$ $(\mathrm{d}, J=7.4 \mathrm{~Hz}, 1 \mathrm{H}), 7.07-6.97(\mathrm{~m}, 6 \mathrm{H}), 6.96-6.89(\mathrm{~m}, 3 \mathrm{H}), 6.44-6.38(\mathrm{~m}, 2 \mathrm{H}), 4.23-4.00(\mathrm{~m}, 2 \mathrm{H})$, $2.15(\mathrm{~s}, 3 \mathrm{H}), 1.17(\mathrm{t}, J=7.1 \mathrm{~Hz}, 3 \mathrm{H})$.

${ }^{13}$ C NMR $\left(101 \mathrm{MHz}, \mathrm{CDCl}_{3}\right) \delta 170.4,165.9,157.6,156.2,138.9,137.6,137.1,136.6,134.3,132.5$, 131.2, 129.0, 128.3, 128.0, 127.5, 126.6, 125.4, 122.4, 118.5, 102.9, 59.7, 20.6, 14.3.

IR (ATR): 2982, 1642, 1590, 1562, 1492, 1438, 1404, 1259, 1210, 1180, 1155, 1092, 1042, 906, $821,796,747,702 \mathrm{~cm}^{-1}$.

HRMS (EI-TOF) m/z calcd for $\mathrm{C}_{28} \mathrm{H}_{24} \mathrm{~N}_{3} \mathrm{O}_{2} \mathrm{Cl}\left[\mathrm{M}^{+}\right]$: 469.1557; found: 469.1552.

\section{Synthetic procedure and Characterization data for 6}

\section{1-((4-Chlorophenyl)(pyrimidin-2-yl)amino)-2-phenyl-3H-pyrrolo[1,2-a]indol-3-one (6a)}<smiles>O=C1C(c2ccccc2)=C(N(c2ccc(Cl)cc2)c2ncccn2)c2cc3ccccc3n21</smiles>

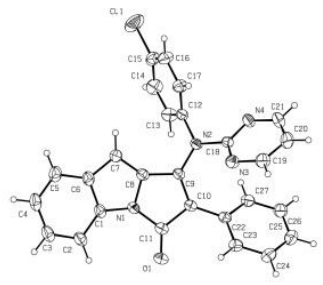

The mixture of $3 \mathbf{a}(0.1 \mathrm{mmol})$, EtONa $(0.5 \mathrm{mmol})$ in $2 \mathrm{~mL}$ of DMSO was stirred at $120{ }^{\circ} \mathrm{C}$ for 2 hours under $\mathrm{N}_{2}$ atmosphere. Then the reaction mixture was poured into $\mathrm{H}_{2} \mathrm{O}(100 \mathrm{~mL})$ and extracted with EtOAc $(3 \times 30 \mathrm{ml})$. The combined organic phase was dried by $\mathrm{Na}_{2} \mathrm{SO}_{4}$. After filtration and evaporation of the solvent under reduce pressure, the crude product was subject to silica gel column chromatography with ethyl acetate/petroleum ether $=1: 4(\mathrm{v}: \mathrm{v})$ as eluent to afford the product. The product $6 \mathbf{a}(44 \mathrm{mg}, 99 \%)$ was obtained as a white solid. m.p. $200-201{ }^{\circ} \mathrm{C}$

${ }^{1} \mathbf{H}$ NMR $\left(400 \mathrm{MHz}, \mathrm{CDCl}_{3}\right) \delta 8.23(\mathrm{~d}, J=4.8 \mathrm{~Hz}, 2 \mathrm{H}), 7.76(\mathrm{~d}, J=8.5 \mathrm{~Hz}, 1 \mathrm{H}), 7.43-7.33(\mathrm{~m}$, $6 \mathrm{H}), 7.30-7.24(\mathrm{~m}, 2 \mathrm{H}), 7.15-7.09(\mathrm{~m}, 3 \mathrm{H}), 7.07-7.03(\mathrm{~m}, 1 \mathrm{H}), 6.68(\mathrm{t}, J=4.8 \mathrm{~Hz}, 1 \mathrm{H}), 5.60(\mathrm{~s}$, $1 \mathrm{H})$.

${ }^{13}$ C NMR $\left(101 \mathrm{MHz}, \mathrm{CDCl}_{3}\right) \delta 164.2,159.5,157.8,142.6,140.2,137.7,133.9,133.3,132.7,131.0$, 129.6, 127.9, 127.8, 127.4, 127.2, 123.0, 122.5, 122.4, 115.0, 112.6, 107.3.

IR (ATR): 3057, 1715, 1563, 1489, 1409, 1370, 1336, 1308, 1153, 1092, 909, 804, 729, 695, 645 $\mathrm{cm}^{-1}$.

HRMS (EI-TOF) m/z calcd for $\mathrm{C}_{27} \mathrm{H}_{17} \mathrm{~N}_{4} \mathrm{OCl}\left[\mathrm{M}^{+}\right]$: 448.1091 ; found: 448.1095 .

1-((4-Chlorophenyl)(pyrimidin-2-yl)amino)-7-methoxy-2-phenyl-3H-pyrrolo[1,2-a]indol-3-one (6b) 


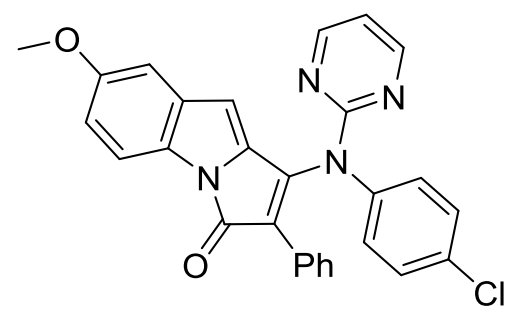

The reaction of $\mathbf{3 c}(0.1 \mathrm{mmol})$, EtONa $(0.5 \mathrm{mmol})$ in DCE $(2 \mathrm{~mL})$ following the typical procedure afforded $\mathbf{6 b}$ (47 mg, $98 \%$ ) after chromatography on silica gel (petroleum ether/ethyl acetate $=4: 1$ ) as a dark red solid; m.p. $201-203{ }^{\circ} \mathrm{C}$.

${ }^{1}$ H NMR (400 MHz, Chloroform- $d$ ) $\delta 8.23(\mathrm{~d}, J=4.8 \mathrm{~Hz}, 2 \mathrm{H}), 7.64(\mathrm{~d}, J=8.7 \mathrm{~Hz}, 1 \mathrm{H}), 7.43-$ $7.32(\mathrm{~m}, 6 \mathrm{H}), 7.13-7.09(\mathrm{~m}, 3 \mathrm{H}), 6.87(\mathrm{dd}, J=8.7,2.4 \mathrm{~Hz}, 1 \mathrm{H}), 6.79(\mathrm{~d}, J=2.3 \mathrm{~Hz}, 1 \mathrm{H}), 6.68(\mathrm{t}, J=$ $4.8 \mathrm{~Hz}, 1 \mathrm{H}), 5.53(\mathrm{~s}, 1 \mathrm{H}), 3.78(\mathrm{~s}, 3 \mathrm{H})$.

${ }^{13}$ C NMR (101 MHz, Chloroform- $d$ ) $\delta$ 164.0, 159.6, 157.8, 156.0, 142.4, 140.2, 138.5, 134.2, 132.7, 131.1, 129.6, 128.8, 127.9, 127.78, 127.75, 127.5, 115.0, 114.9, 113.0, 107.1, 106.2, 55.7.

IR (film): 2925, 2852, 1716, 1574, 1490, 1477, 1408, 1373, 1314, 1252, 1220, 1153, 1092, 911, $847,805,734,696 \mathrm{~cm}^{-1}$.

HRMS (EI-TOF) m/z calcd for $\mathrm{C}_{28} \mathrm{H}_{19} \mathrm{~N}_{4} \mathrm{OCl}\left[\mathrm{M}^{+}\right]$: 478.1197; found: 478.1194 .

\section{7-Bromo-1-((4-chlorophenyl)(pyrimidin-2-yl)amino)-2-phenyl-3H-pyrrolo[1,2-a]indol-3-one (6c)}<smiles>O=C1C(c2ccccc2)=C(N(c2ccc(Cl)cc2)c2ncccn2)c2cc3cc(Br)ccc3n21</smiles>

The reaction of $\mathbf{3 d}(0.1 \mathrm{mmol})$, EtONa $(0.5 \mathrm{mmol})$ in DCE $(2 \mathrm{~mL})$ following the typical procedure afforded 6c (52 mg, $99 \%$ ) after chromatography on silica gel (petroleum ether/ethyl acetate $=4: 1)$ as a dark red solid; m.p. $237-239^{\circ} \mathrm{C}$.

${ }^{1}$ H NMR (400 MHz, Chloroform- $\left.d\right) \delta 8.23(\mathrm{~d}, J=4.8 \mathrm{~Hz}, 2 \mathrm{H}), 7.64(\mathrm{~d}, J=8.4 \mathrm{~Hz}, 1 \mathrm{H}), 7.45-$ $7.31(\mathrm{~m}, 8 \mathrm{H}), 7.16-7.06(\mathrm{~m}, 3 \mathrm{H}), 6.70(\mathrm{t}, J=4.8 \mathrm{~Hz}, 1 \mathrm{H}), 5.51(\mathrm{~s}, 1 \mathrm{H})$.

${ }^{13}$ C NMR (101 MHz, Chloroform- $d$ ) $\delta 164.0,159.4,157.8,142.5,140.0,138.7,134.9,133.0,132.4$, $130.9,129.8,129.7,128.0,127.91,127.85,127.5,125.1,122.4,115.9,115.2,113.7,106.1$

IR (film): 2970, 2923, 1721, 1561, 1490, 1408, 1372, 1320, 1089, 1049, 803, 740, $695 \mathrm{~cm}^{-1}$.

HRMS (EI-TOF) $\mathrm{m} / \mathrm{z}$ calcd for $\mathrm{C}_{27} \mathrm{H}_{16} \mathrm{~N}_{4} \mathrm{OBrCl}\left[\mathrm{M}^{+}\right]$: 526.0196; found: 526.0195.

1-((4-Chlorophenyl)(pyrimidin-2-yl)amino)-2-(4-methoxyphenyl)-3H-pyrrolo[1,2-a]indol-3-one (6d)<smiles>COc1ccc(C2=C(N(c3ccc(Cl)cc3)c3ncccn3)c3cc4ccccc4n3C2=O)cc1</smiles> 
The reaction of $\mathbf{3 f}(0.1 \mathrm{mmol})$, EtONa $(0.5 \mathrm{mmol})$ in DCE $(2 \mathrm{~mL})$ following the typical procedure afforded 6d (43 mg, $90 \%$ ) after chromatography on silica gel (petroleum ether/ethyl acetate $=4: 1$ ) as a dark red solid; m.p. $212-213{ }^{\circ} \mathrm{C}$.

${ }^{1} \mathbf{H}$ NMR $\left(400 \mathrm{MHz}, \mathrm{CDCl}_{3}\right) \delta 8.27(\mathrm{~d}, J=4.8 \mathrm{~Hz}, 2 \mathrm{H}), 7.75(\mathrm{~d}, J=8.1 \mathrm{~Hz}, 1 \mathrm{H}), 7.40-7.33(\mathrm{~m}$, $6 \mathrm{H}), 7.29-7.23(\mathrm{~m}, 2 \mathrm{H}), 7.06-7.02(\mathrm{~m}, 1 \mathrm{H}), 6.72(\mathrm{t}, J=4.8 \mathrm{~Hz}, 1 \mathrm{H}), 6.70-6.63(\mathrm{~m}, 2 \mathrm{H}), 5.62(\mathrm{~s}$, $1 \mathrm{H}), 3.74(\mathrm{~s}, 3 \mathrm{H})$.

${ }^{13}$ C NMR $\left(101 \mathrm{MHz}, \mathrm{CDCl}_{3}\right) \delta 164.4,159.6,159.3,157.9,141.1,140.1,138.1,133.9,133.5,132.5$, $129.5,128.8,127.5,127.0,123.5,123.1,123.0,122.4,115.0,113.4,112.5,106.8,55.2$.

IR (ATR): 3057, 2837, 1717, 1609, 1567, 1509, 1490, 1411, 1372, 1305, 1251, 1178, 1093, 1033, $911,842,798,734 \mathrm{~cm}^{-1}$.

HRMS (EI-TOF) m/z calcd for $\mathrm{C}_{28} \mathrm{H}_{19} \mathrm{~N}_{4} \mathrm{O}_{2} \mathrm{Cl}\left[\mathrm{M}^{+}\right]$: 478.1197 ; found: 478.1198 .

\section{2-(4-Bromophenyl)-1-((4-chlorophenyl)(pyrimidin-2-yl)amino)-3H-pyrrolo[1,2-a]indol-3-one (6e)}

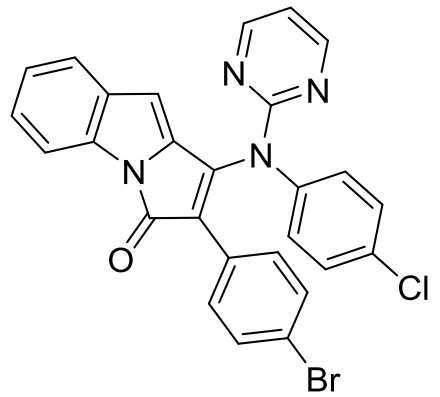

The reaction of $3 \mathbf{e}(0.1 \mathrm{mmol}), \mathrm{EtONa}(0.5 \mathrm{mmol})$ in DCE $(2 \mathrm{~mL})$ following the typical procedure afforded $6 \mathbf{e}(52 \mathrm{mg}, 99 \%)$ after chromatography on silica gel (petroleum ether/ethyl acetate $=4: 1)$ as a dark red solid; m.p. $226-228^{\circ} \mathrm{C}$.

${ }^{1}$ H NMR (400 MHz, Chloroform- $d$ ) $\delta 8.28(\mathrm{~d}, J=4.8 \mathrm{~Hz}, 2 \mathrm{H}), 7.75(\mathrm{~d}, J=8.4 \mathrm{~Hz}, 1 \mathrm{H}), 7.44-$ $7.40(\mathrm{~m}, 2 \mathrm{H}), 7.39-7.35(\mathrm{~m}, 2 \mathrm{H}), 7.31-7.22(\mathrm{~m}, 6 \mathrm{H}), 7.10-7.02(\mathrm{~m}, 1 \mathrm{H}), 6.75(\mathrm{t}, J=4.8 \mathrm{~Hz}, 1 \mathrm{H})$, $5.59(\mathrm{~d}, J=0.8 \mathrm{~Hz}, 1 \mathrm{H})$.

${ }^{13}$ C NMR (101 MHz, Chloroform- $d$ ) $\delta$ 163.8, 159.6, 157.9, 142.9, 140.1, 137.5, 133.9, 133.3, 133.0, 131.0, 130.1, 129.7, 128.9, 127.8, 127.4, 123.1, 122.6, 121.8, 121.0, 115.4, 112.6, 107.8.

IR (film): 2923, 2852, 1716, 1561, 1489, 1409, 1397, 1372, 1335, 1312, 1156, 1091, 1010, 966, $909,819,730 \mathrm{~cm}^{-1}$.

HRMS (EI-TOF) m/z calcd for $\mathrm{C}_{27} \mathrm{H}_{16} \mathrm{~N}_{4} \mathrm{OBrCl}\left[\mathrm{M}^{+}\right]$: 526.0196; found: 526.0198.

\section{2-Phenyl-1-(pyrimidin-2-yl(4-(trifluoromethyl)phenyl)amino)-3H-pyrrolo[1,2-a]indol-3-one (6f)}<smiles>O=C1C(c2ccccc2)=C(N(c2ccc(C(F)(F)F)cc2)c2ncccn2)c2cc3ccccc3n21</smiles>

The reaction of $\mathbf{3 g}(0.1 \mathrm{mmol})$, EtONa $(0.5 \mathrm{mmol})$ in DCE $(2 \mathrm{~mL})$ following the typical procedure afforded $6 \mathbf{f}$ (35 mg, $73 \%$ ) after chromatography on silica gel (petroleum ether/ethyl acetate $=4: 1$ ) as a dark red solid; m.p. $238-240{ }^{\circ} \mathrm{C}$. 
${ }^{1} \mathbf{H}$ NMR $\left(500 \mathrm{MHz}, \mathrm{CDCl}_{3}\right) \delta 8.28(\mathrm{~d}, J=4.8 \mathrm{~Hz}, 2 \mathrm{H}), 7.77(\mathrm{~d}, J=8.2 \mathrm{~Hz}, 1 \mathrm{H}), 7.66(\mathrm{~d}, J=8.4$ $\mathrm{Hz}, 2 \mathrm{H}), 7.54$ (d, J = 8.3 Hz, 2H), $7.41-7.35$ (m, 2H), $7.31-7.24(\mathrm{~m}, 2 \mathrm{H}), 7.15-7.09$ (m, 3H), 7.06 $(\mathrm{t}, J=7.3 \mathrm{~Hz}, 1 \mathrm{H}), 6.74(\mathrm{t}, J=4.8 \mathrm{~Hz}, 1 \mathrm{H}), 5.64(\mathrm{~s}, 1 \mathrm{H})$.

${ }^{13}$ C NMR $\left(126 \mathrm{MHz}, \mathrm{CDCl}_{3}\right) \delta 163.9,159.5,157.9,144.6,142.4,137.6,134.0,133.3,130.6,128.5$ $(\mathrm{q}, J=32.9 \mathrm{~Hz}), 128.0,127.9,127.6,127.3,126.4(\mathrm{q}, J=3.7 \mathrm{~Hz}), 126.1,124.2,123.8(\mathrm{q}, J=270.1 \mathrm{~Hz})$, 123.1, 122.6, 115.5, 112.6, 107.2.

IR (ATR): 3057, 1718, 1610, 1564, 1408, 1371, 1320, 1163, 1121, 1067, 910, 806, 747, $696 \mathrm{~cm}^{-1}$.

HRMS (EI-TOF) m/z calcd for $\mathrm{C}_{28} \mathrm{H}_{17} \mathrm{~F}_{3} \mathrm{~N}_{4} \mathrm{O}\left[\mathrm{M}^{+}\right]$: 482.1354; found: 482.1349 .

\section{2-Phenyl-1-(pyrimidin-2-yl(p-tolyl)amino)-3H-pyrrolo[1,2-a]indol-3-one $(6 \mathrm{~g})$}<smiles>Cc1ccc(N(C2=C(c3ccccc3)C(=O)n3c2cc2ccccc23)c2ncccn2)cc1</smiles>

The reaction of $\mathbf{3 h}(0.1 \mathrm{mmol})$, EtONa $(0.5 \mathrm{mmol})$ in DCE $(2 \mathrm{~mL})$ following the typical procedure afforded $\mathbf{6 g}$ (42 mg, $98 \%$ ) after chromatography on silica gel (petroleum ether/ethyl acetate $=4: 1$ ) as a dark red solid; m.p. $257-258^{\circ} \mathrm{C}$.

${ }^{1}$ H NMR (400 MHz, Chloroform- $d$ ) $\delta 8.18(\mathrm{~d}, J=4.8 \mathrm{~Hz}, 2 \mathrm{H}), 7.76(\mathrm{~d}, J=8.4 \mathrm{~Hz}, 1 \mathrm{H}), 7.41-$ $7.32(\mathrm{~m}, 4 \mathrm{H}), 7.30-7.20(\mathrm{~m}, 4 \mathrm{H}), 7.14-7.05(\mathrm{~m}, 3 \mathrm{H}), 7.04-7.00(\mathrm{~m}, 1 \mathrm{H}), 6.61(\mathrm{t}, J=4.8 \mathrm{~Hz}, 1 \mathrm{H})$, $5.49(\mathrm{~d}, J=0.9 \mathrm{~Hz}, 1 \mathrm{H}), 2.42(\mathrm{~s}, 3 \mathrm{H})$.

${ }^{13}$ C NMR (101 MHz, Chloroform- $d$ ) $\delta 164.5,159.8,157.6,143.2,139.2,138.0,137.5,133.8,133.3$, 131.6, 130.2, 127.8, 127.40, 127.36, 127.0, 126.7, 122.7, 122.4, 120.7, 114.6, 112.5, 107.3, 21.2.

IR (film): 2923, 2853, 1713, 1580, 1563, 1510, 1408, 1367, 1335, 1310, 1154, 1105, 816, 805, 763 $\mathrm{cm}^{-1}$.

HRMS (EI-TOF) m/z calcd for $\mathrm{C}_{28} \mathrm{H}_{20} \mathrm{~N}_{4} \mathrm{O}\left[\mathrm{M}^{+}\right]$: 428.1637; found: 428.1634 .

\section{1-((4-Methoxyphenyl)(pyrimidin-2-yl)amino)-2-phenyl-3H-pyrrolo[1,2-a]indol-3-one (6h)}

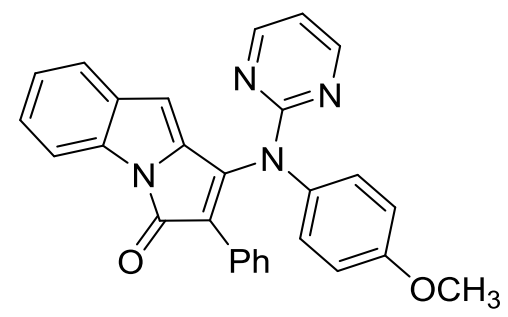

The reaction of $3 \mathbf{i}(0.1 \mathrm{mmol})$, EtONa $(0.5 \mathrm{mmol})$ in DCE $(2 \mathrm{~mL})$ following the typical procedure afforded $\mathbf{6 h}$ (38 mg, $86 \%$ ) after chromatography on silica gel (petroleum ether/ethyl acetate $=4: 1)$ as a dark red solid; m.p. $214-216{ }^{\circ} \mathrm{C}$.

${ }^{1} \mathbf{H}$ NMR $\left(500 \mathrm{MHz}, \mathrm{CDCl}_{3}\right) \delta 8.18(\mathrm{~d}, J=4.8 \mathrm{~Hz}, 2 \mathrm{H}), 7.76(\mathrm{~d}, J=8.4 \mathrm{~Hz}, 1 \mathrm{H}), 7.41-7.33(\mathrm{~m}$, $4 \mathrm{H}), 7.28-7.21(\mathrm{~m}, 2 \mathrm{H}), 7.14-7.06(\mathrm{~m}, 3 \mathrm{H}), 7.05-7.01(\mathrm{~m}, 1 \mathrm{H}), 7.01-6.96(\mathrm{~m}, 2 \mathrm{H}), 6.61(\mathrm{t}, J=$ $4.8 \mathrm{~Hz}, 1 \mathrm{H}), 5.49(\mathrm{~s}, 1 \mathrm{H}), 3.86(\mathrm{~s}, 3 \mathrm{H})$.

${ }^{13}$ C NMR $\left(126 \mathrm{MHz}, \mathrm{CDCl}_{3}\right) \delta 164.5,159.8,158.8,157.5,143.3,138.0,134.6,133.8,133.3,131.7$, $128.2,127.8,127.4,127.0,122.7,122.4,120.2,114.8,114.5,112.5,107.3,55.5$. 
IR (ATR): 3054, 2837, 1713, 1607, 1561, 1508, 1408, 1371, 1299, 1248, 1154, 1105, 1032, 910, $846,806,744,696 \mathrm{~cm}^{-1}$.

HRMS (EI-TOF) m/z calcd for $\mathrm{C}_{28} \mathrm{H}_{20} \mathrm{~N}_{4} \mathrm{O}_{2}\left[\mathrm{M}^{+}\right]$: 444.1586 ; found: 444.1584 .

\section{1-((3-Chlorophenyl)(pyrimidin-2-yl)amino)-2-phenyl-3H-pyrrolo[1,2-a]indol-3-one (6i)}

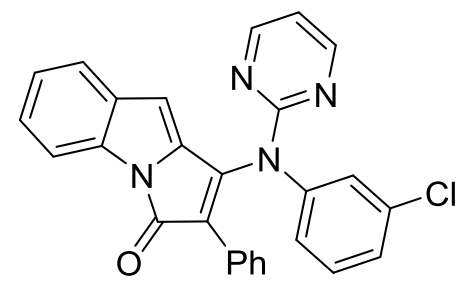

The reaction of $\mathbf{3 j}(0.1 \mathrm{mmol})$, EtONa $(0.5 \mathrm{mmol})$ in DCE $(2 \mathrm{~mL})$ following the typical procedure afforded $\mathbf{6 i}$ (42 mg, $94 \%$ ) after chromatography on silica gel (petroleum ether/ethyl acetate $=4: 1$ ) as a dark red solid; m.p. $201-202{ }^{\circ} \mathrm{C}$.

${ }^{1}$ H NMR (400 MHz, Chloroform- $d$ ) $\delta 8.24(\mathrm{~d}, J=4.8 \mathrm{~Hz}, 2 \mathrm{H}), 7.76(\mathrm{~d}, J=8.5 \mathrm{~Hz}, 1 \mathrm{H}), 7.43-$ $7.42(\mathrm{~m}, 1 \mathrm{H}), 7.39-7.23(\mathrm{~m}, 7 \mathrm{H}), 7.15-7.08(\mathrm{~m}, 3 \mathrm{H}), 7.07-7.01(\mathrm{~m}, 1 \mathrm{H}), 6.69(\mathrm{t}, J=4.8 \mathrm{~Hz}, 1 \mathrm{H})$, $5.62(\mathrm{~s}, 1 \mathrm{H})$.

${ }^{13}$ C NMR (101 MHz, Chloroform- $d$ ) $\delta$ 164.04, 159.47, 157.78, 142.68, 142.52, 137.65, 134.80, $133.87,133.30,130.85,130.23,127.78,127.49,127.20,127.15,126.61,124.64,122.96,122.82$, $122.46,115.17,112.52,107.22$.

IR (film): 3057, 2925, 1716, 1575, 1475, 1447, 1409, 1372, 1336, 1312, 1156, 1096, 1077, 971, $909,812,732,695,646 \mathrm{~cm}^{-1}$.

HRMS (EI-TOF) m/z calcd for $\mathrm{C}_{27} \mathrm{H}_{17} \mathrm{~N}_{4} \mathrm{OCl}\left[\mathrm{M}^{+}\right]$: 448.1091 ; found: 448.1088 .

\section{1-(Ethyl(pyrimidin-2-yl)amino)-2-phenyl-3H-pyrrolo[1,2-a]indol-3-one (6j)}

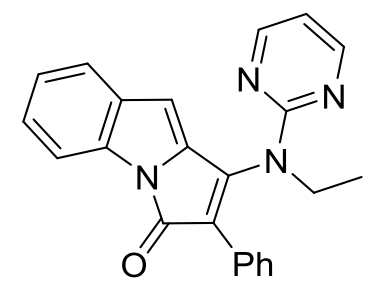

The reaction of $31(0.1 \mathrm{mmol})$, EtONa $(0.5 \mathrm{mmol})$ in DCE $(2 \mathrm{~mL})$ following the typical procedure afforded $\mathbf{6 j}$ (32 mg, $87 \%$ ) after chromatography on silica gel (petroleum ether/ethyl acetate $=4: 1$ ) as a dark red solid; m.p. $192-194{ }^{\circ} \mathrm{C}$.

${ }^{1}$ H NMR (400 MHz, Chloroform- $d$ ) $\delta 8.36(\mathrm{~d}, J=4.7 \mathrm{~Hz}, 2 \mathrm{H}), 7.78(\mathrm{~d}, J=8.0 \mathrm{~Hz}, 1 \mathrm{H}), 7.57-$ $7.50(\mathrm{~m}, 2 \mathrm{H}), 7.36(\mathrm{~d}, J=7.8 \mathrm{~Hz}, 1 \mathrm{H}), 7.34-7.22(\mathrm{~m}, 4 \mathrm{H}), 7.10-7.06(\mathrm{~m}, 1 \mathrm{H}), 6.71(\mathrm{t}, J=4.8 \mathrm{~Hz}$, 1H), $6.22(\mathrm{~s}, 1 \mathrm{H}), 3.94(\mathrm{q}, J=7.0 \mathrm{~Hz}, 2 \mathrm{H}), 1.29(\mathrm{t}, J=7.0 \mathrm{~Hz}, 3 \mathrm{H})$.

${ }^{13}$ C NMR (101 MHz, Chloroform- $\left.d\right) \delta 164.4,160.1,157.8,144.1,138.8,134.0,133.6,130.8$, 128.22, 128.20, 128.17, 127.1, 124.6, 123.0, 122.4, 113.1, 112.7, 106.4, 43.9, 13.4 .

IR (film): 2926, 2852, 1716, 1576, 1557, 1468, 1449, 1422, 1380, 1365, 1292, 1165, 1088, 799, $747,694 \mathrm{~cm}^{-1}$.

HRMS (EI-TOF) m/z calcd for $\mathrm{C}_{23} \mathrm{H}_{18} \mathrm{~N}_{4} \mathrm{O}\left[\mathrm{M}^{+}\right]$: 366.1481 ; found: 366.1483 .

1-(Isopropyl(pyrimidin-2-yl)amino)-2-phenyl-3H-pyrrolo[1,2-a]indol-3-one (6k) 


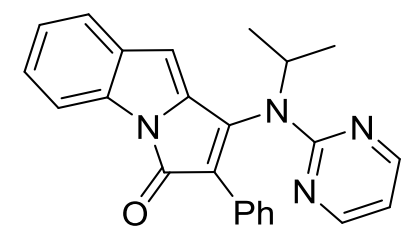

The reaction of $\mathbf{3 m}(0.1 \mathrm{mmol})$, EtONa $(0.5 \mathrm{mmol})$ in DCE $(2 \mathrm{~mL})$ following the typical procedure afforded 6k (34 mg, $89 \%$ ) after chromatography on silica gel (petroleum ether/ethyl acetate = 4:1) as a dark red solid; m.p. $147-148^{\circ} \mathrm{C}$.

${ }^{1}$ H NMR (400 MHz, Chloroform- $\left.d\right) \delta 8.40(\mathrm{~d}, J=4.8 \mathrm{~Hz}, 2 \mathrm{H}), 7.77(\mathrm{dd}, J=8.0,0.9 \mathrm{~Hz}, 1 \mathrm{H}), 7.59$ $-7.52(\mathrm{~m}, 2 \mathrm{H}), 7.37-7.24(\mathrm{~m}, 5 \mathrm{H}), 7.10-7.06(\mathrm{~m}, 1 \mathrm{H}), 6.70(\mathrm{t}, J=4.8 \mathrm{~Hz}, 1 \mathrm{H}), 6.15(\mathrm{~d}, J=0.7 \mathrm{~Hz}$, 1H), 4.79 (hept, $J=6.8 \mathrm{~Hz}, 1 \mathrm{H}), 1.21$ (d, $J=6.8 \mathrm{~Hz}, 6 \mathrm{H})$.

${ }^{13}$ C NMR (101 MHz, Chloroform- $d$ ) $\delta$ 164.0, 160.9, 157.9, 142.7, 140.1, 134.4, 133.8, 131.4, 130.6, $128.6,128.5,128.2,127.1,123.2,122.5,112.7,112.4,106.4,50.6,20.6$.

IR (film): 3055, 2975, 2931, 1722, 1581, 1553, 1447, 1434, 1385, 1361, 1339, 1297, 1160, 1142, 959, 797, 747, 694, $645 \mathrm{~cm}^{-1}$.

HRMS (EI-TOF) m/z calcd for $\mathrm{C}_{24} \mathrm{H}_{20} \mathrm{~N}_{4} \mathrm{O}\left[\mathrm{M}^{+}\right]$: 380.1637 ; found: 380.1634 .

\section{1-(Cyclohexyl(pyrimidin-2-yl)amino)-2-phenyl-3H-pyrrolo[1,2-a]indol-3-one (6I)}<smiles>O=C1C(c2ccccc2)=C(N(c2ncccn2)C2CCCCC2)c2cc3ccccc3n21</smiles>

The reaction of $3 \mathbf{n}(0.1 \mathrm{mmol})$, EtONa $(0.5 \mathrm{mmol})$ in DCE $(2 \mathrm{~mL})$ following the typical procedure afforded $\mathbf{6 l}$ (40 mg, $95 \%)$ after chromatography on silica gel (petroleum ether/ethyl acetate = 4:1) as an orange oil.

${ }^{1} \mathbf{H}$ NMR $\left(500 \mathrm{MHz}, \mathrm{CDCl}_{3}\right) \delta 8.39(\mathrm{~d}, J=4.8 \mathrm{~Hz}, 2 \mathrm{H}), 7.76(\mathrm{~d}, J=7.9 \mathrm{~Hz}, 1 \mathrm{H}), 7.55(\mathrm{dd}, J=7.8$, $1.9 \mathrm{~Hz}, 2 \mathrm{H}), 7.34(\mathrm{~d}, J=7.7 \mathrm{~Hz}, 1 \mathrm{H}), 7.34-7.23(\mathrm{~m}, 4 \mathrm{H}), 7.10-7.06(\mathrm{~m}, 1 \mathrm{H}), 6.67(\mathrm{t}, J=4.8 \mathrm{~Hz}, 1 \mathrm{H})$, $6.16(\mathrm{~s}, 1 \mathrm{H}), 4.51-4.44(\mathrm{~m}, 1 \mathrm{H}), 1.77-1.67(\mathrm{~m}, 4 \mathrm{H}), 1.57-1.55(\mathrm{~m}, 1 \mathrm{H}), 1.50-1.42(\mathrm{~m}, 2 \mathrm{H}), 1.35-$ $1.21(\mathrm{~m}, 2 \mathrm{H}), 1.04-0.96(\mathrm{~m}, 1 \mathrm{H})$.

${ }^{13}$ C NMR $\left(126 \mathrm{MHz}, \mathrm{CDCl}_{3}\right) \delta 163.9,161.0,158.0,142.5,140.3,134.4,133.8,132.0,130.6$, $128.53,128.52,128.2,127.1,123.2,122.4,112.7,112.2,106.4,58.0,30.9,26.0,25.4$.

IR (ATR): 2932, 2856, 1721, 1609, 1580, 1433, 1361, 1297, 1157, 962, 909, 799, 746, $694 \mathrm{~cm}^{-1}$.

HRMS (EI-TOF) m/z calcd for $\mathrm{C}_{27} \mathrm{H}_{24} \mathrm{~N}_{4} \mathrm{O}\left[\mathrm{M}^{+}\right]$: 420.1950 ; found: 420.1945 . 


\section{Copies of NMR Spectra}

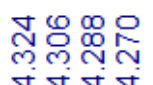

ن̛ं

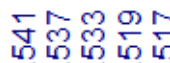

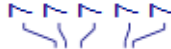

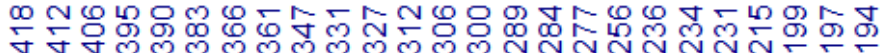

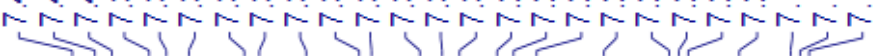
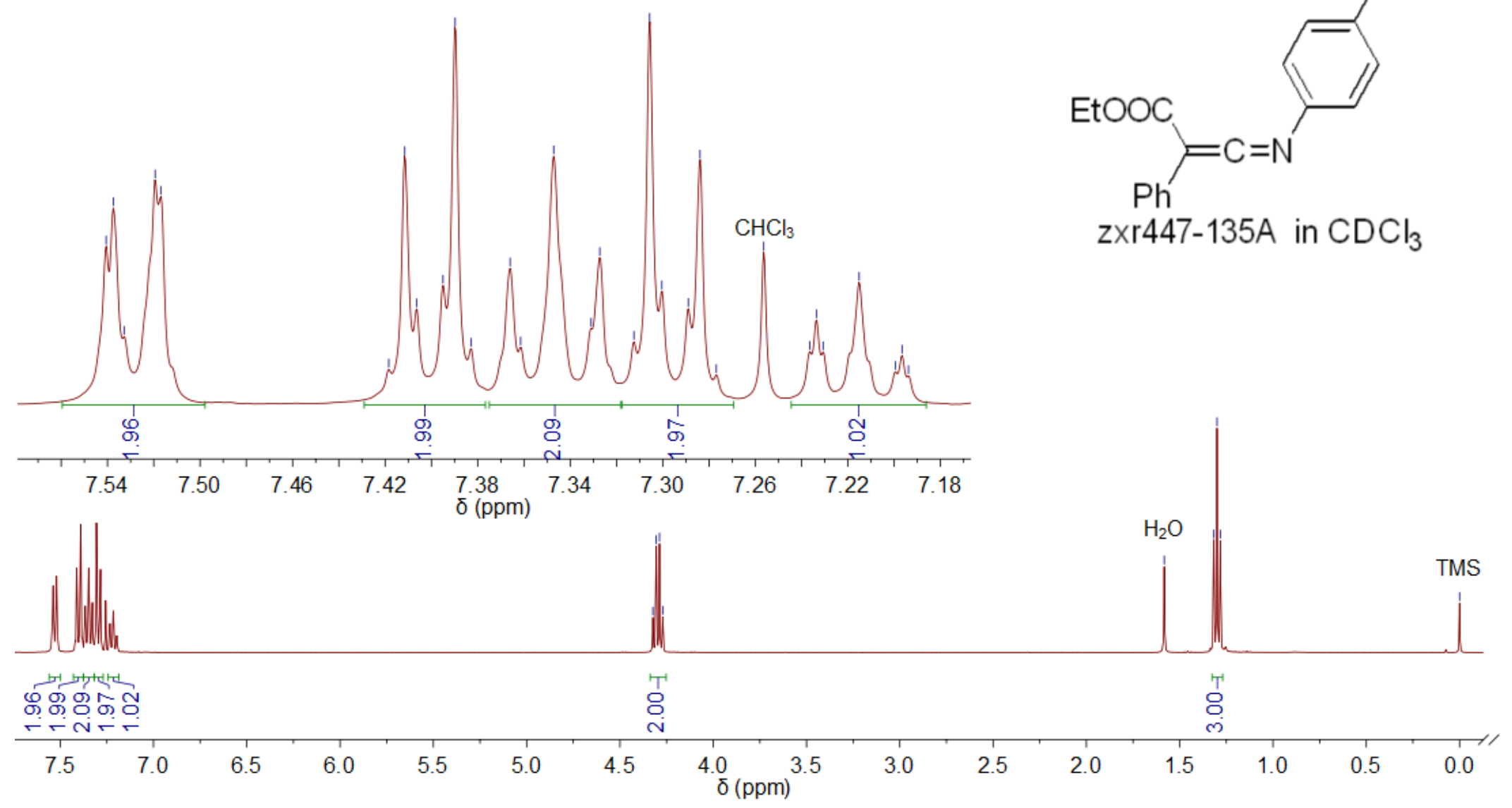

2a $-{ }^{1} \mathrm{H}$ NMR

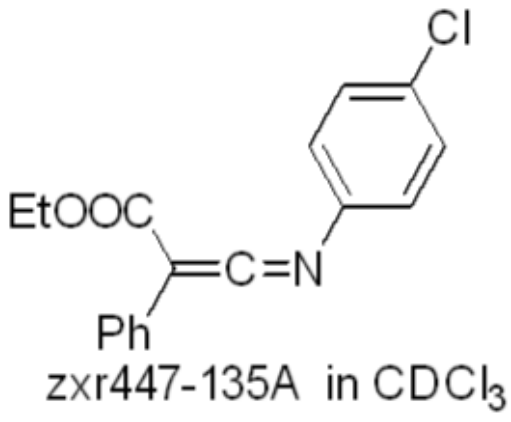

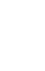




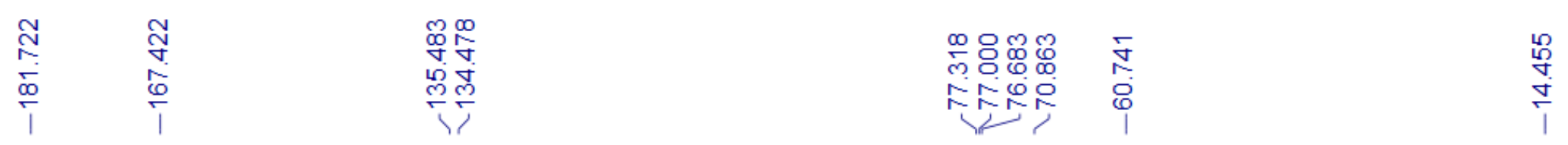

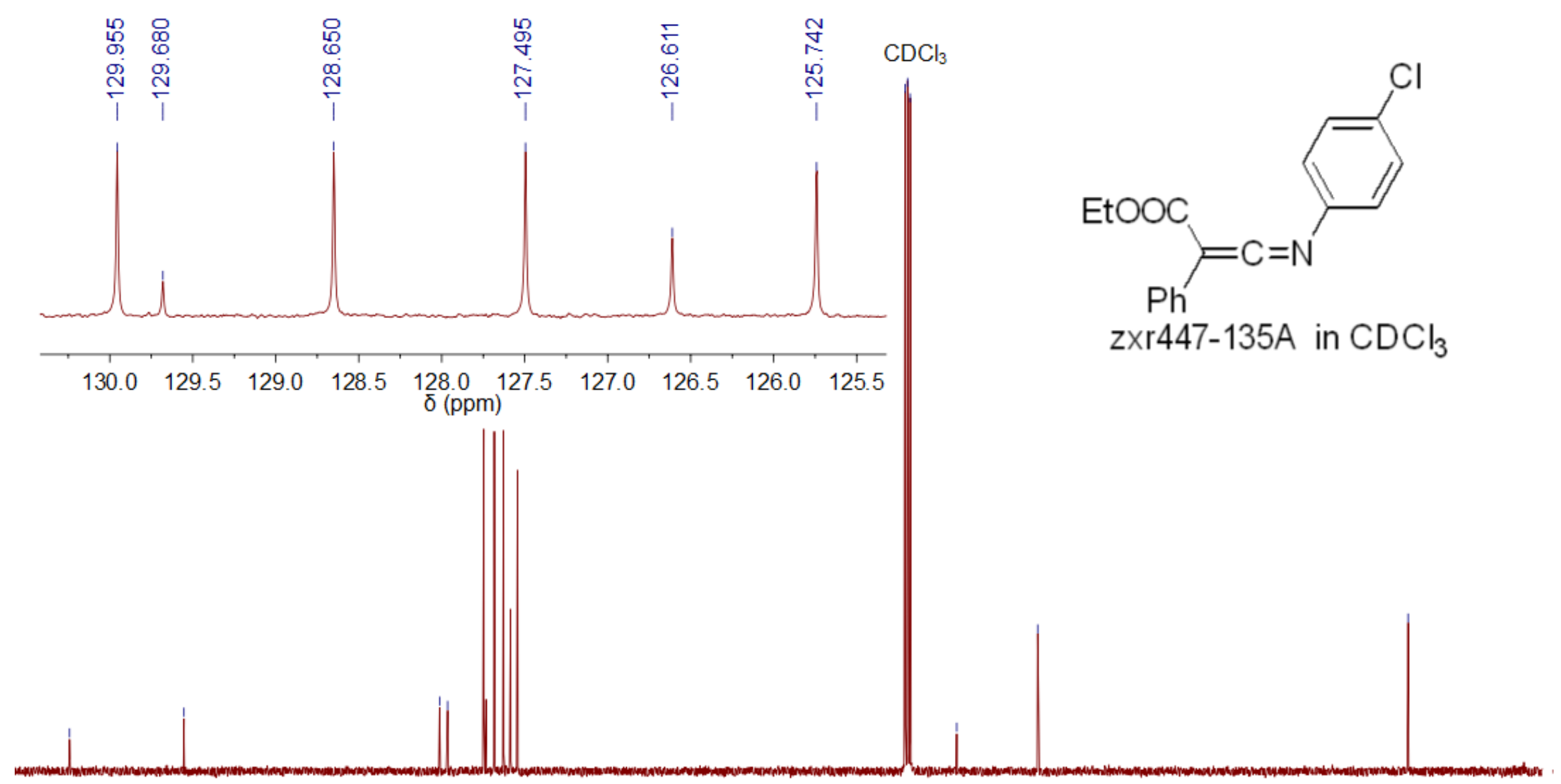

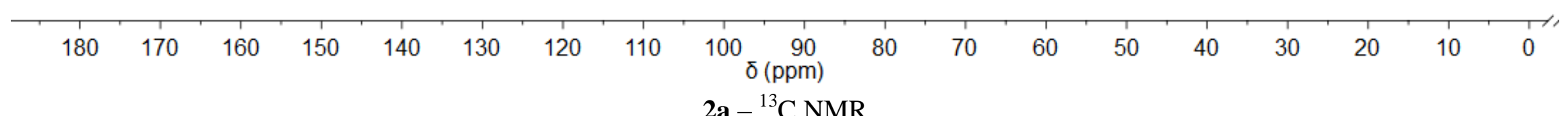


ํํำ

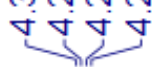

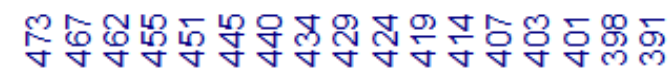

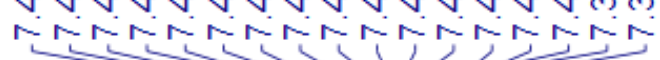

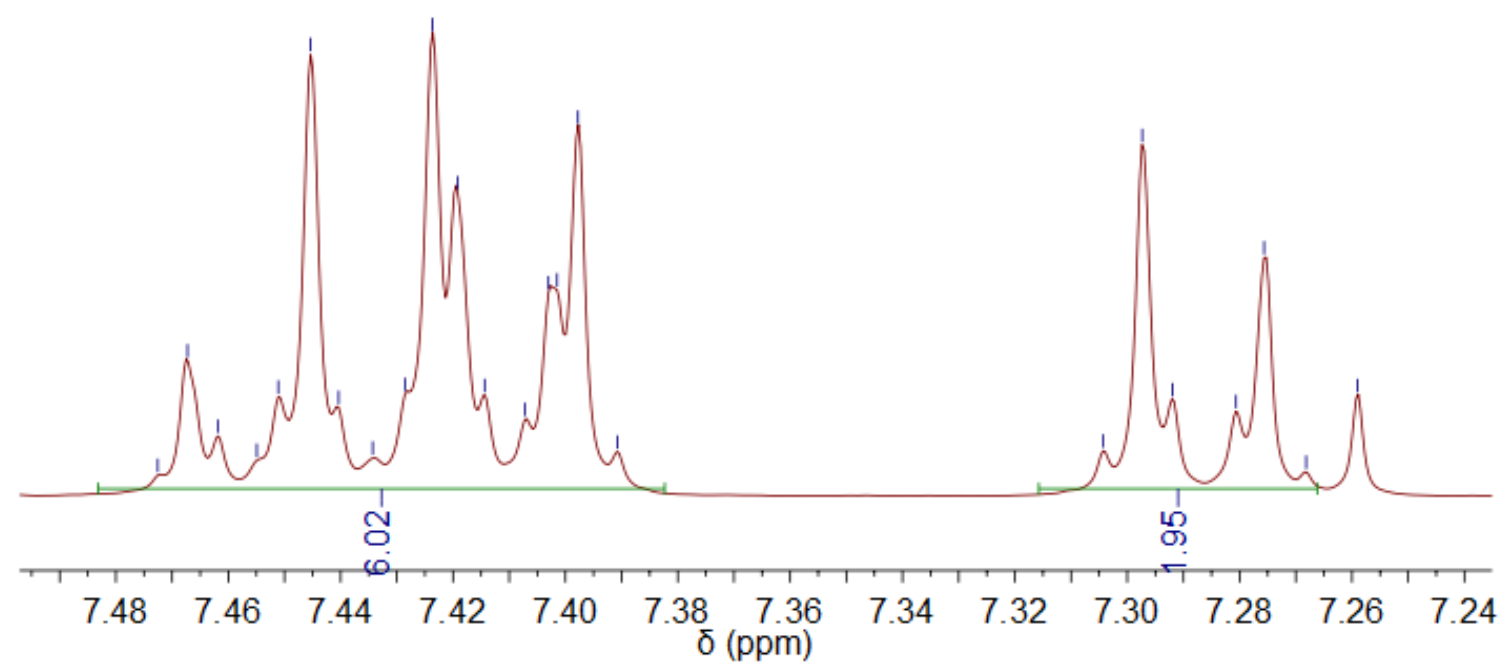

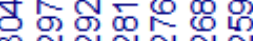

Tiñu

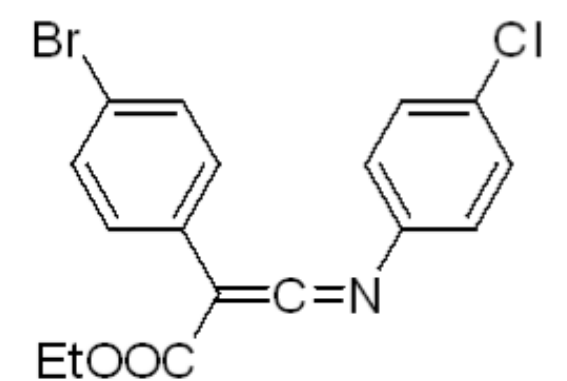

zxr447-135B in $\mathrm{CDCl}_{3}$

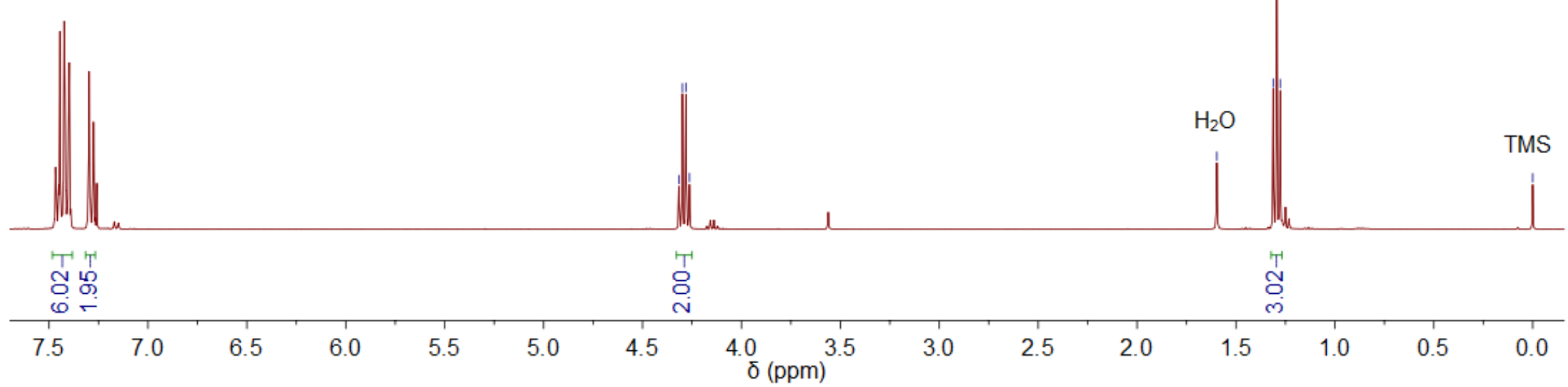

2b $-{ }^{1} \mathrm{H}$ NMR 

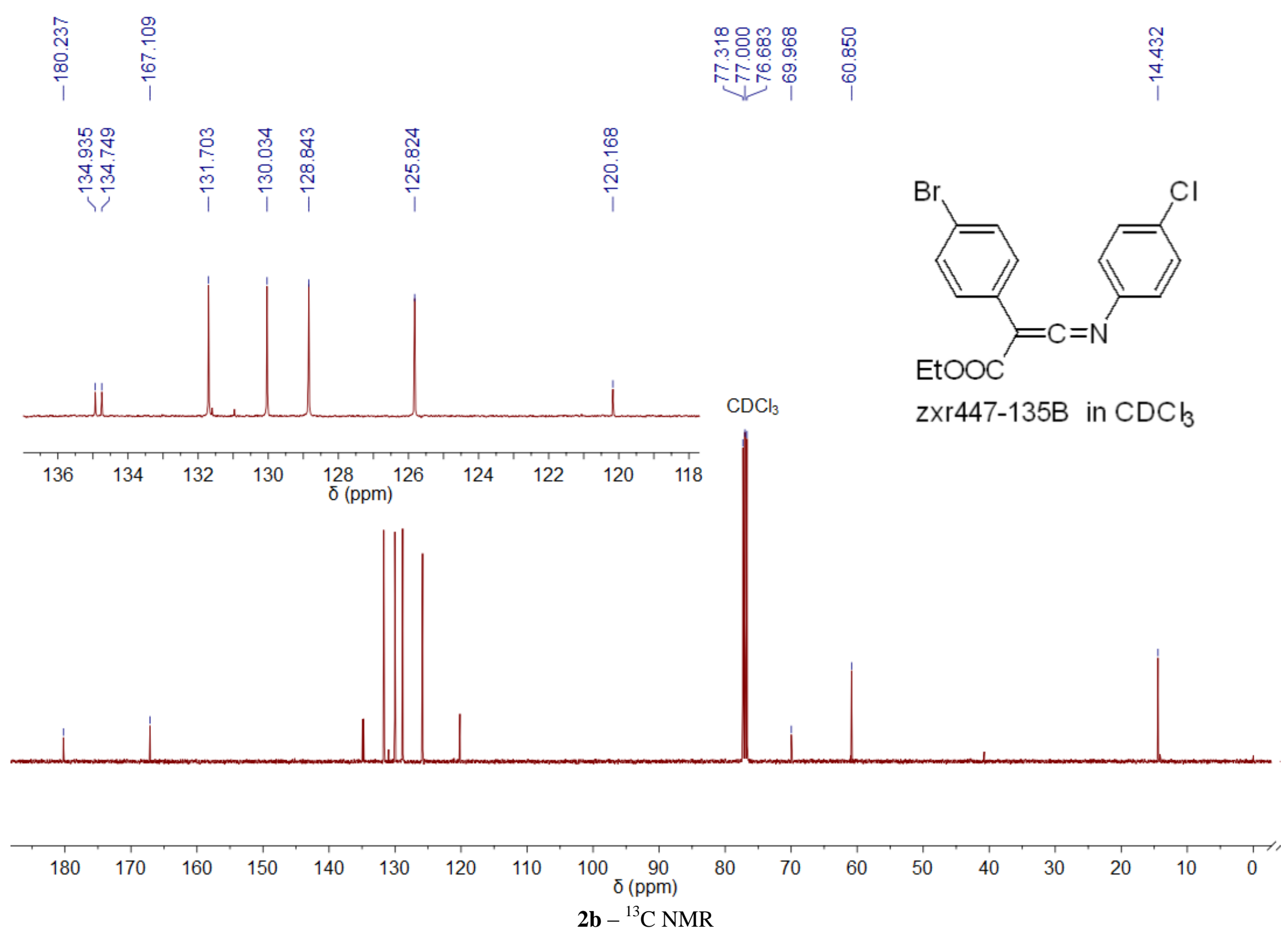


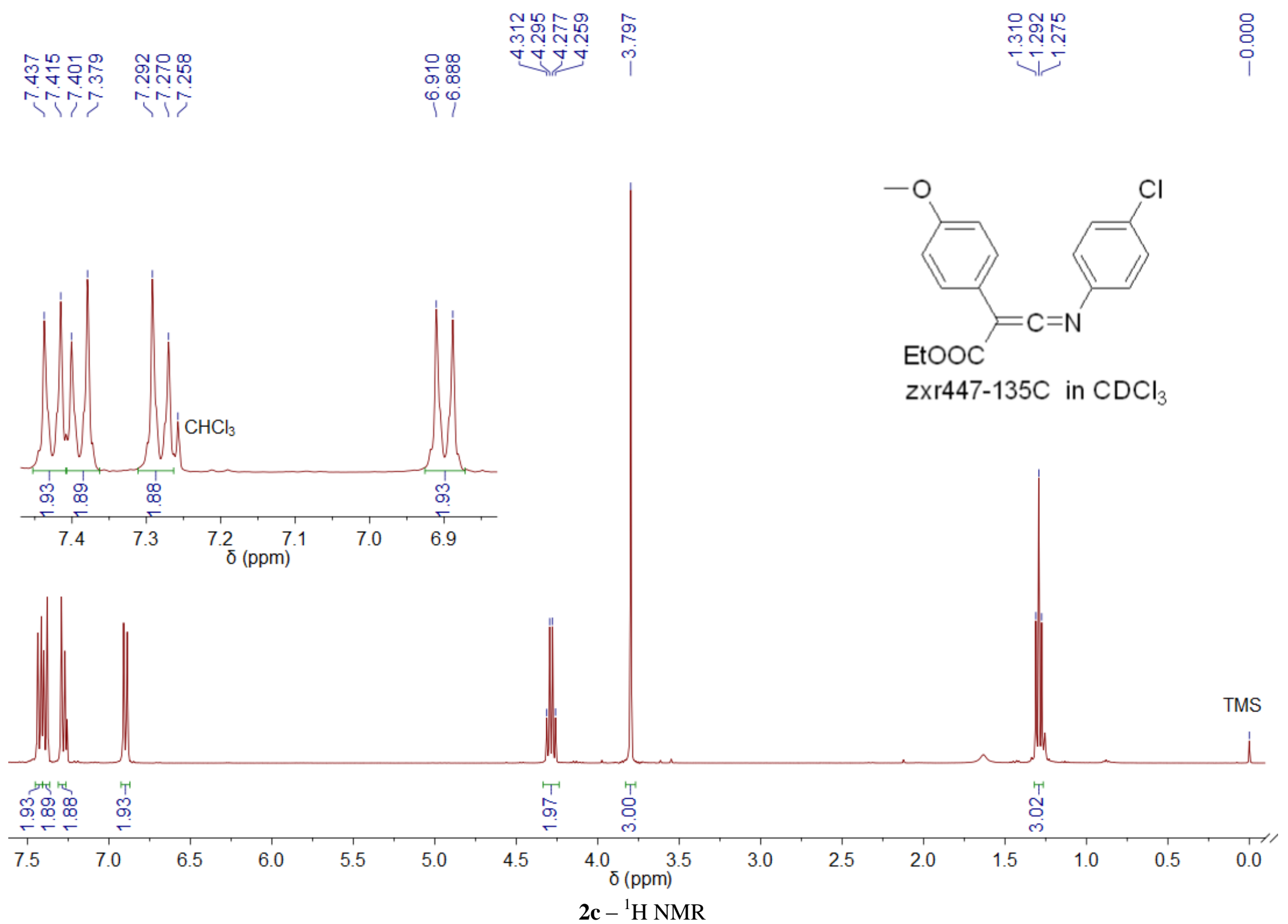



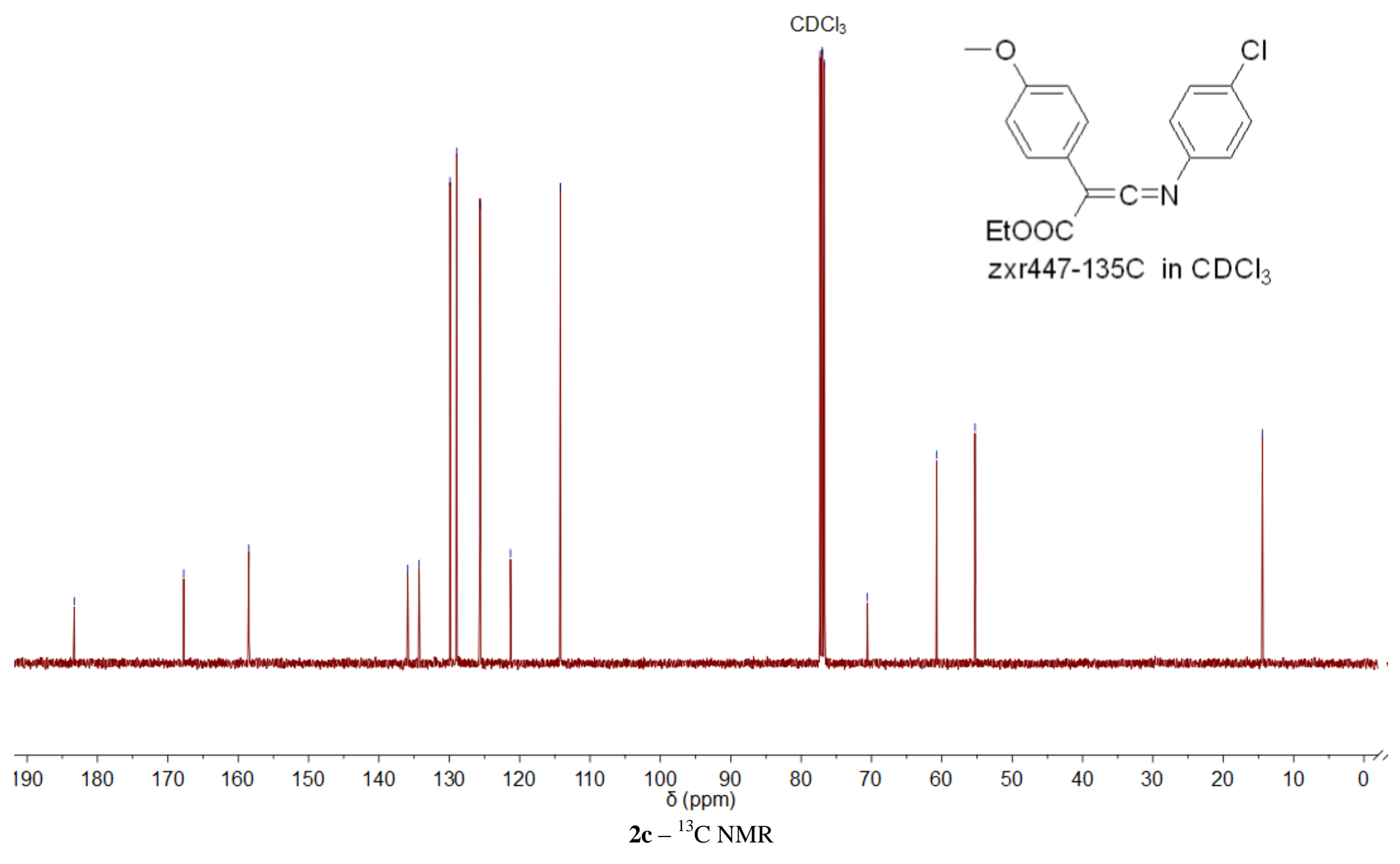


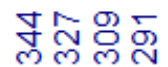 \\ نु过}

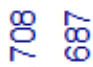

i

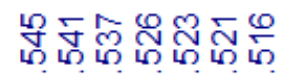

Nivinis

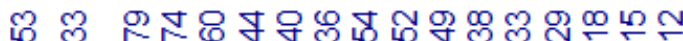

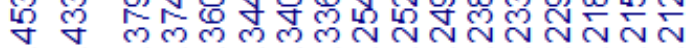

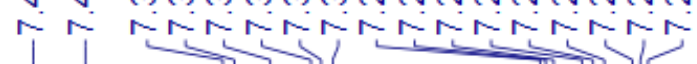
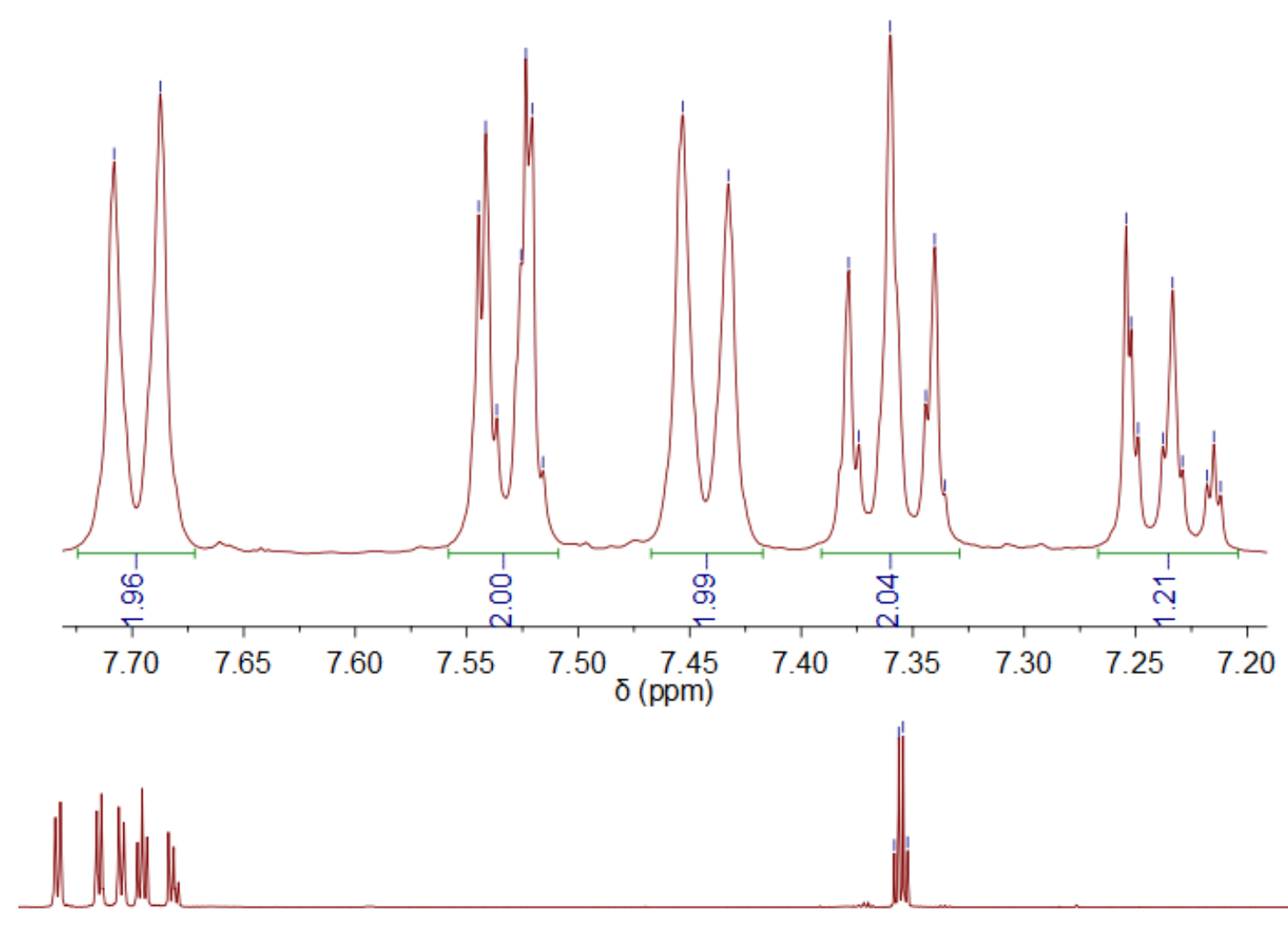

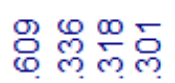

15

o

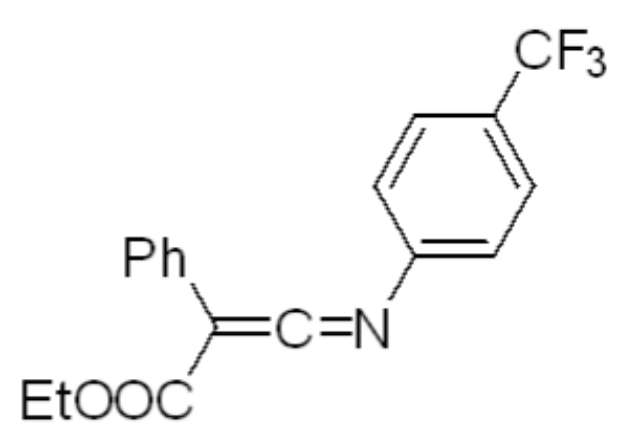

zxr447-157C in $\mathrm{CDCl}_{3}$

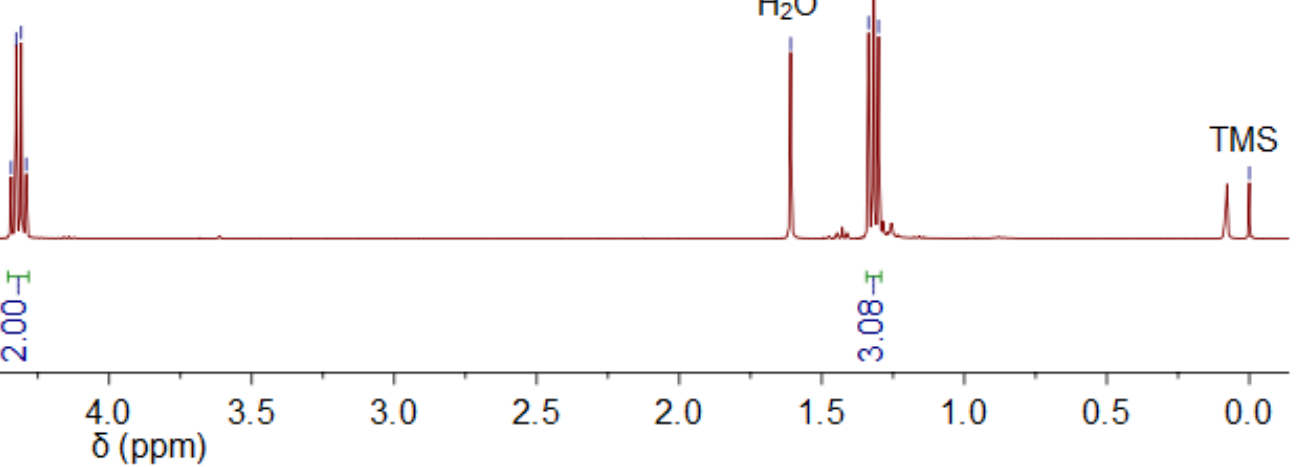

2d $-{ }^{1} \mathrm{H}$ NMR 


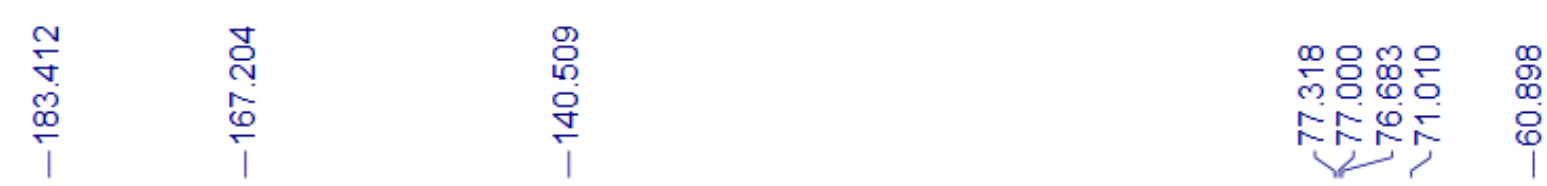
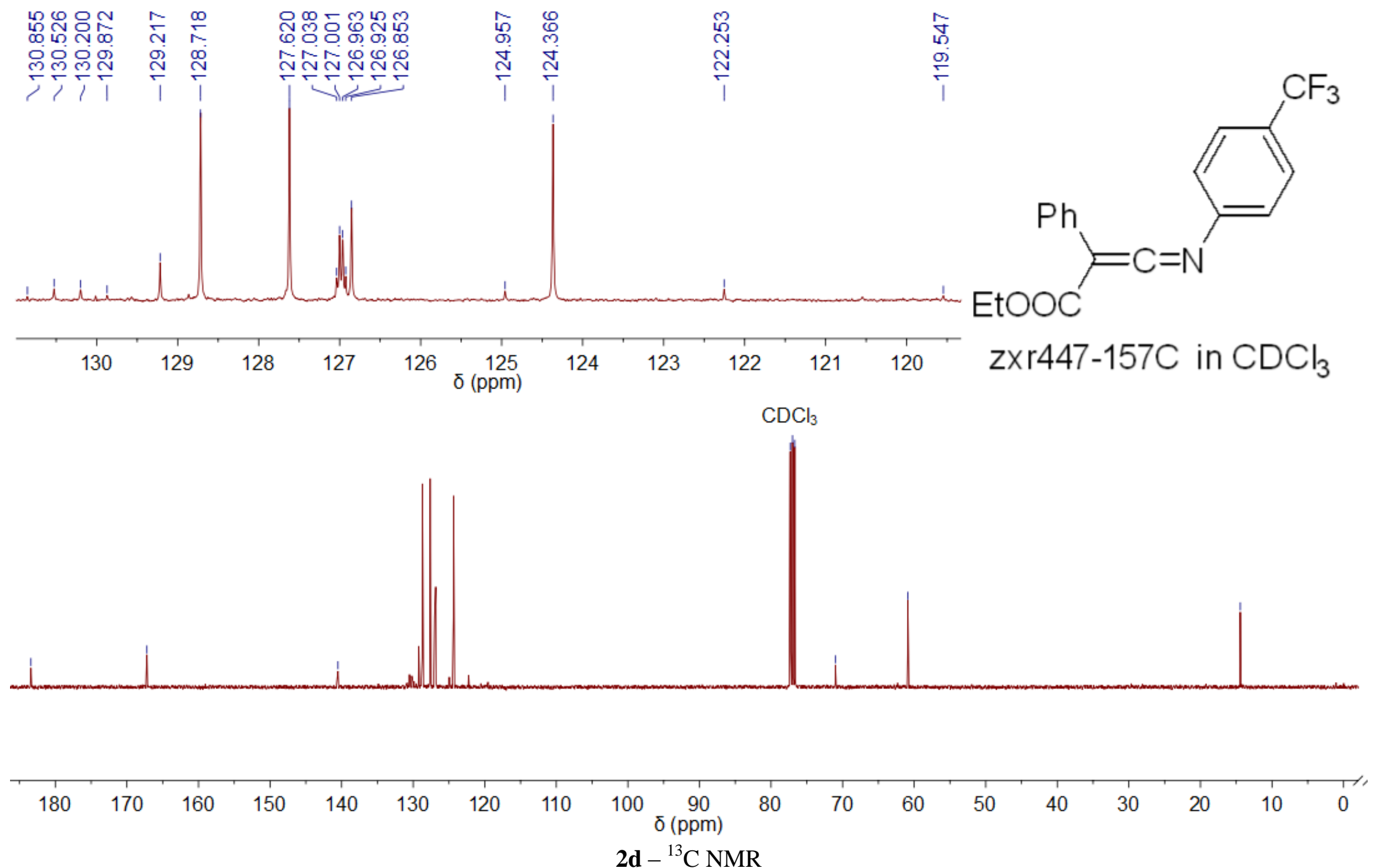


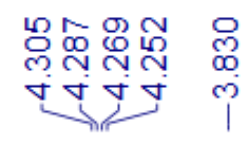

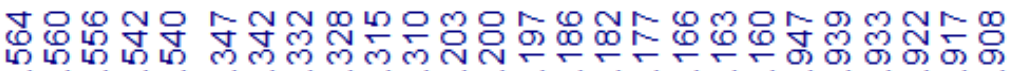

Nin
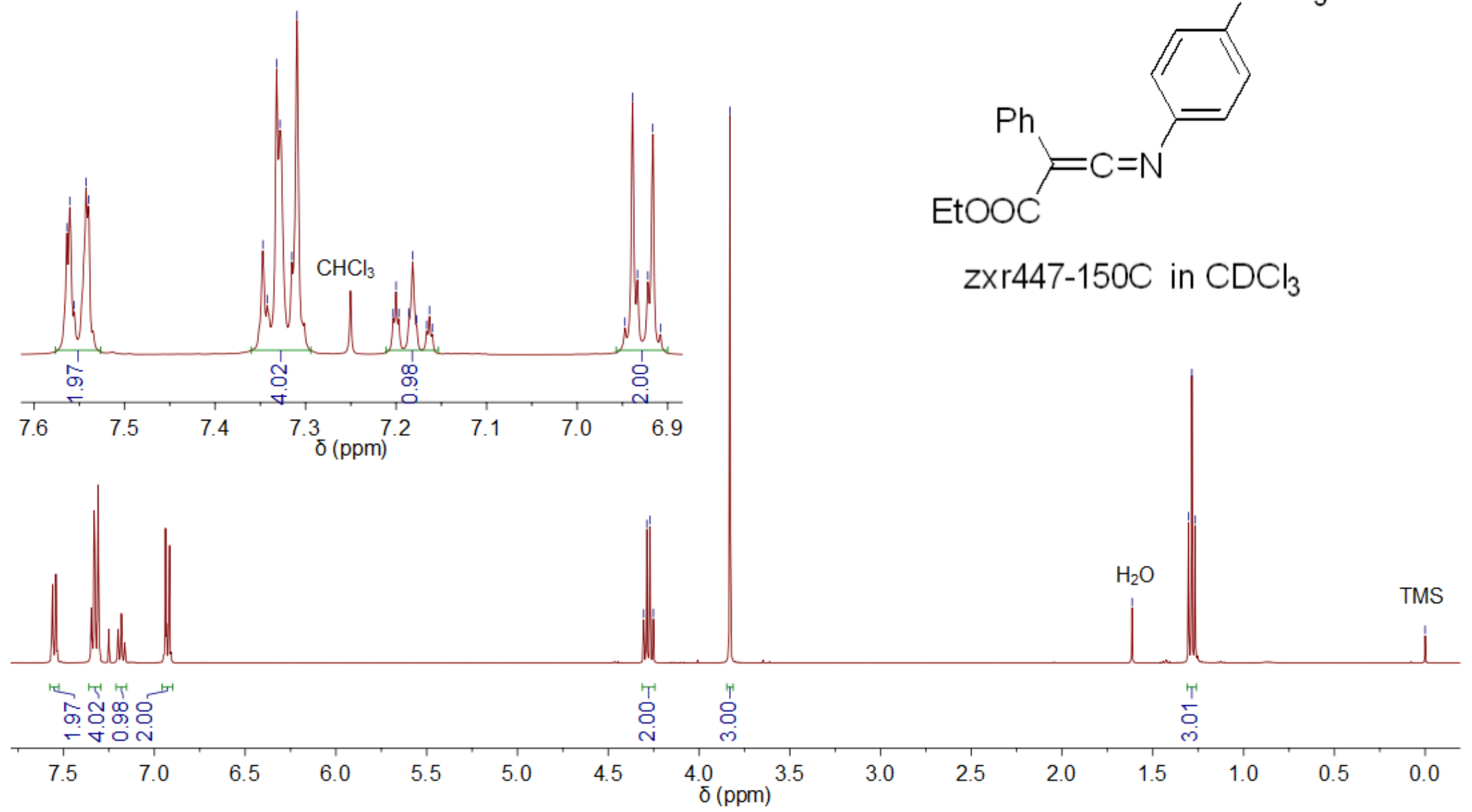

2f $-{ }^{1} \mathrm{H}$ NMR

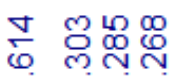

广

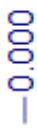

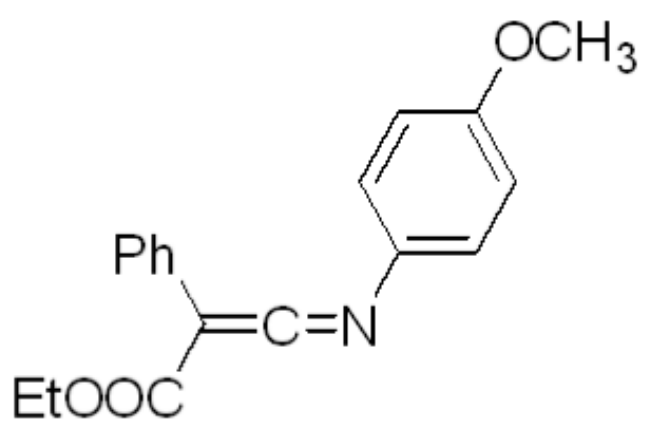

zxr447-150C in $\mathrm{CDCl}_{3}$

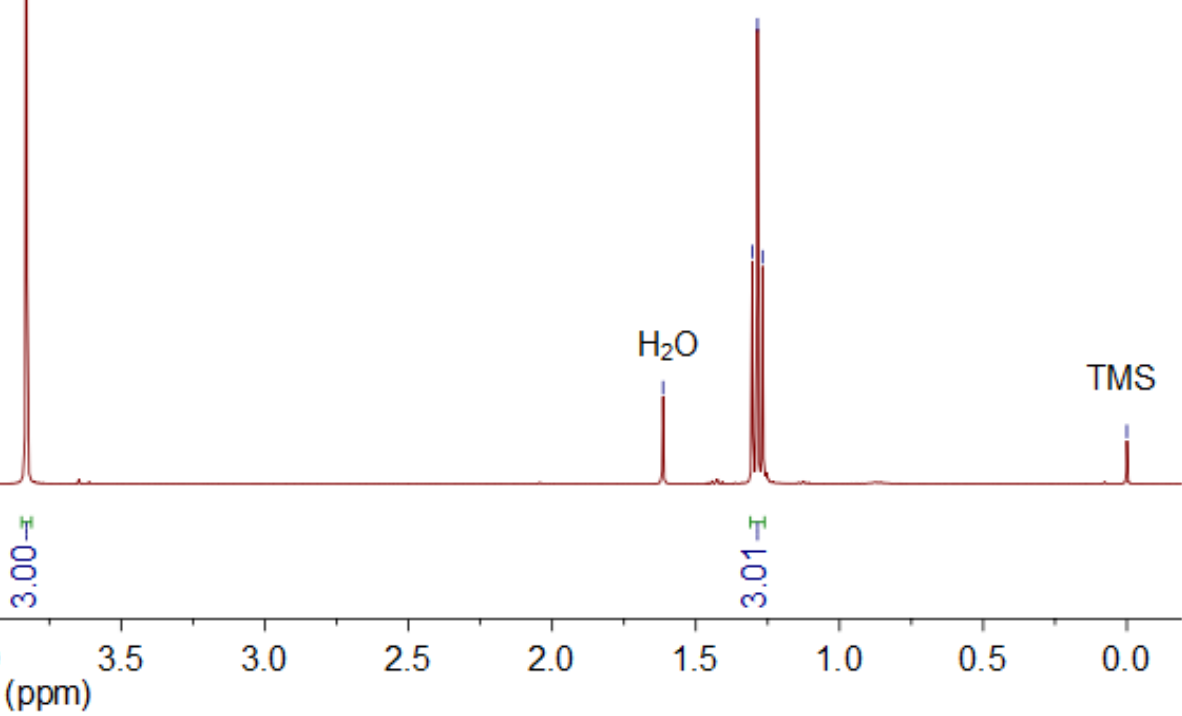

S31 


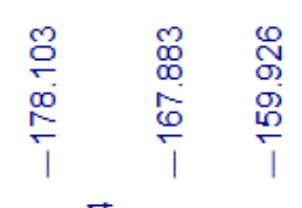

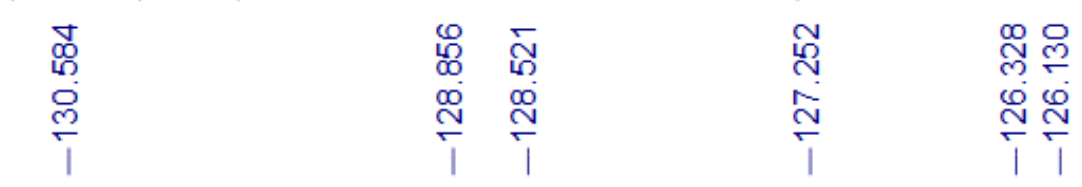

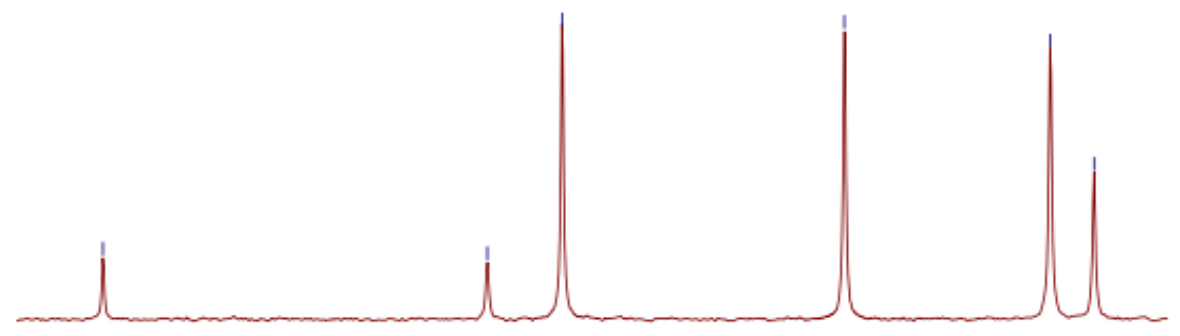

응이용

№

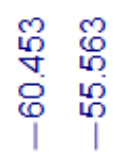

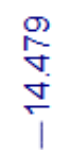

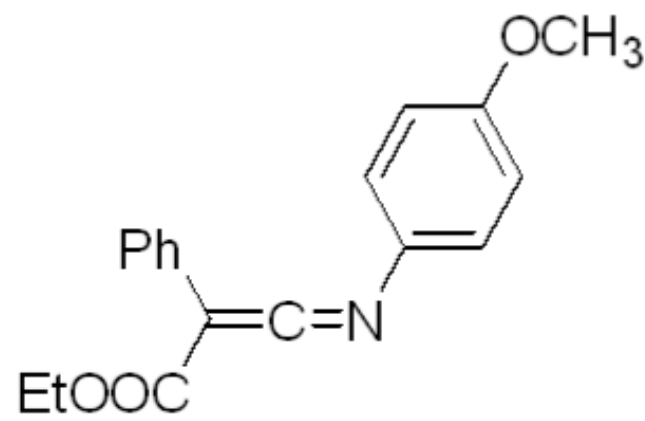

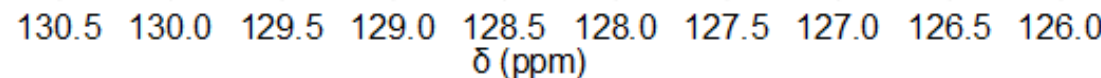

$\mathrm{CDCl}_{3}$
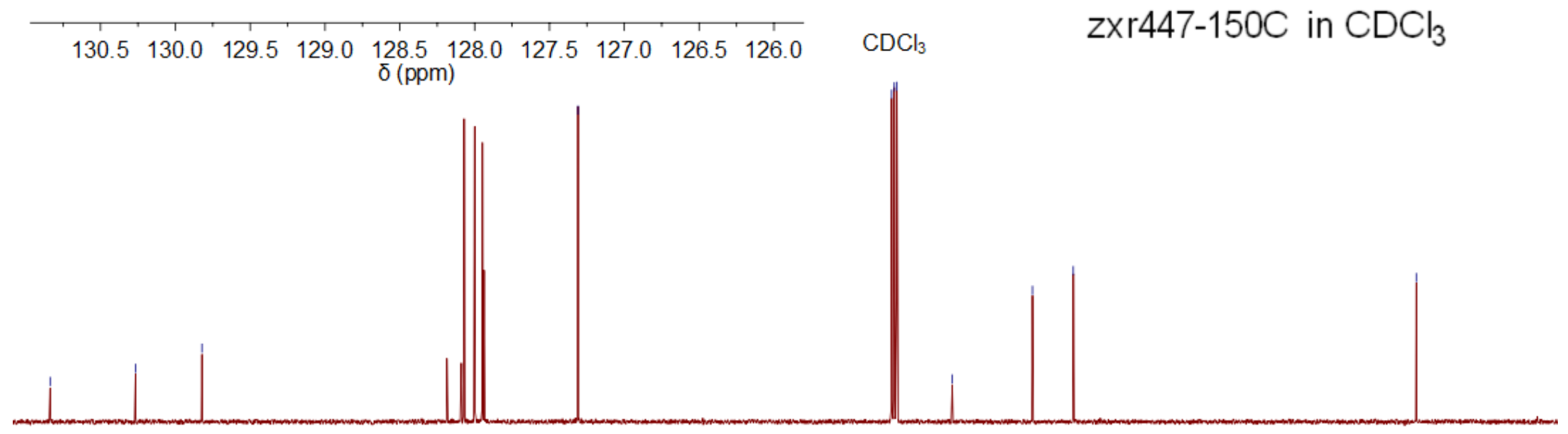

$180 \quad 170 \quad 160$

150

140

130

120

90

80

70

60

50

40

30

20

2f $-{ }^{13} \mathrm{C}$ NMR 


$$
\begin{aligned}
& \text { 임 } \\
& \text { ن̛ं̛ }
\end{aligned}
$$

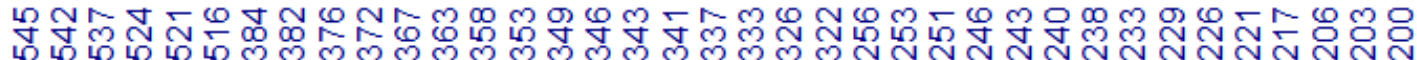
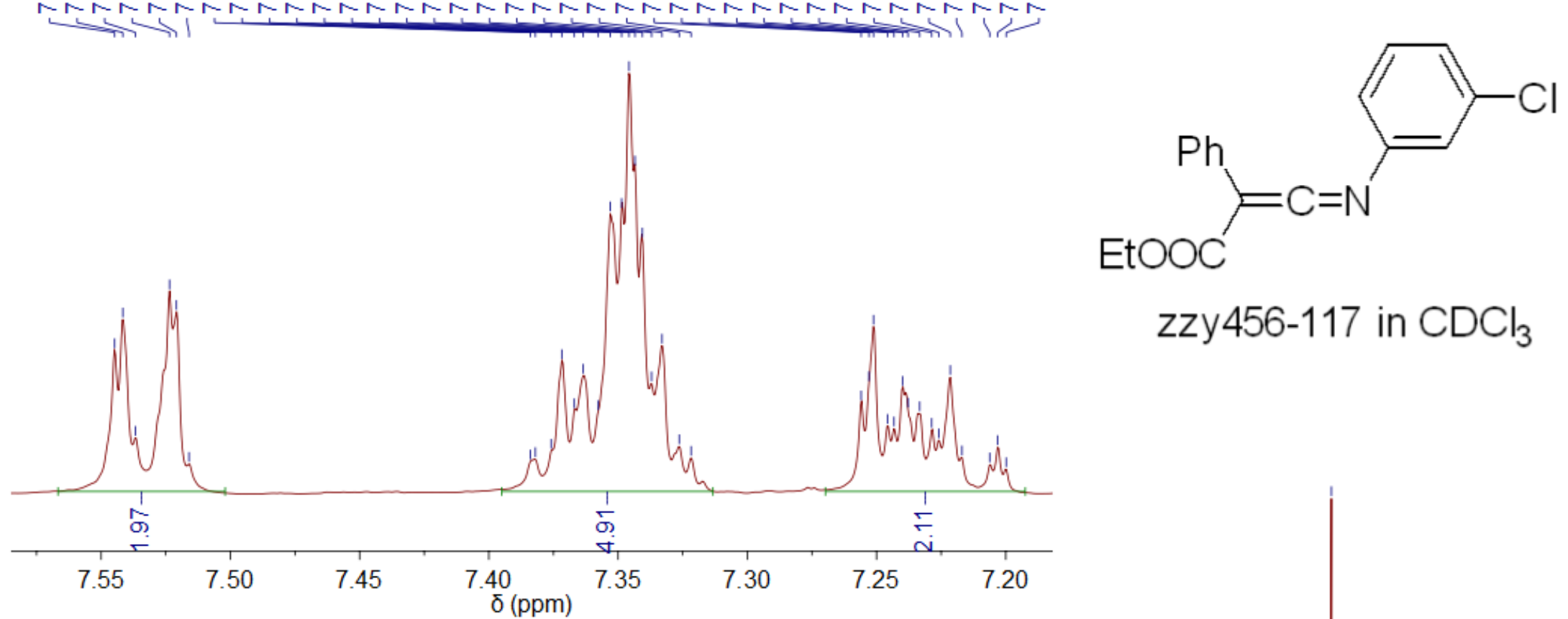

\section{zzy456-117 in $\mathrm{CDCl}_{3}$}
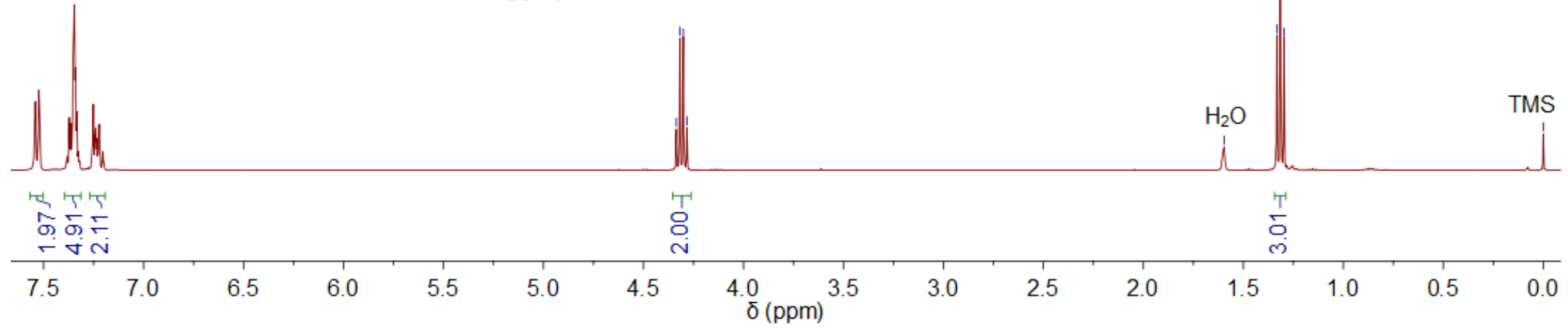

2g - ${ }^{1} \mathrm{H}$ NMR 


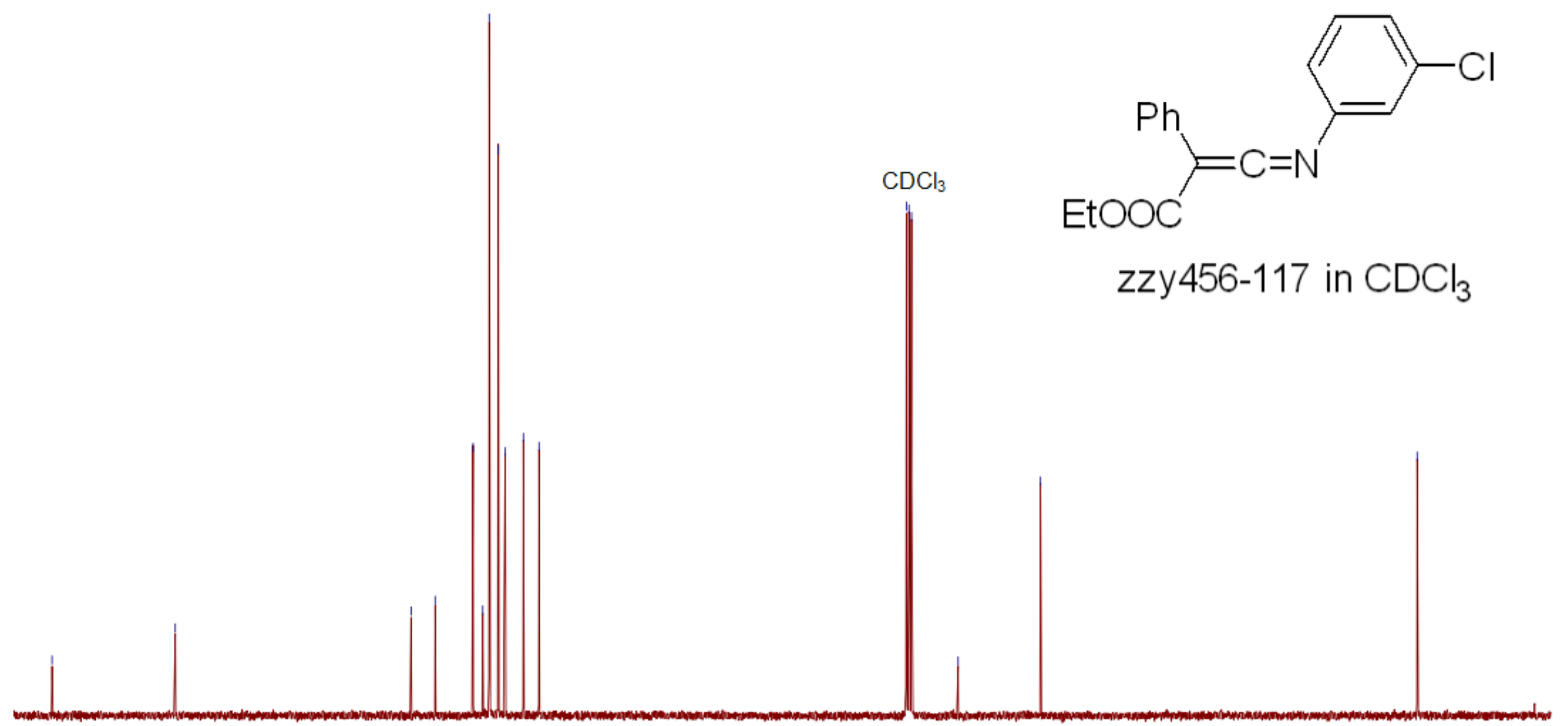

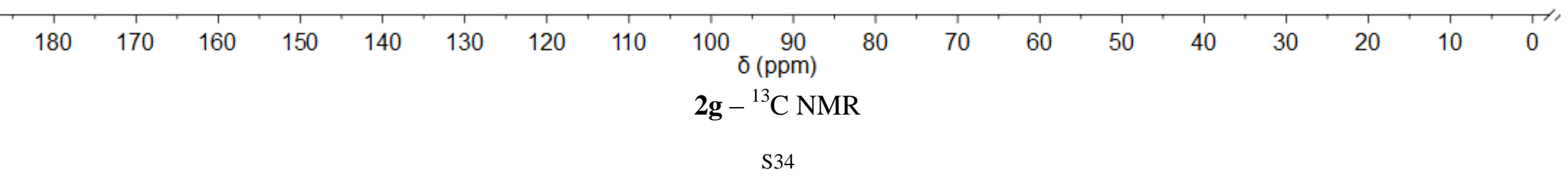




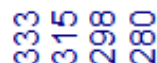

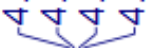

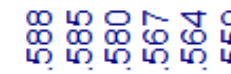

$\min$

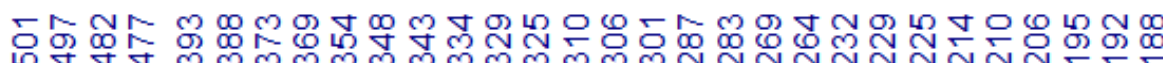

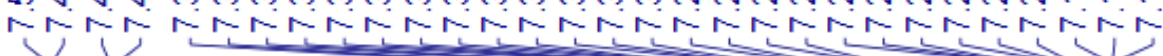

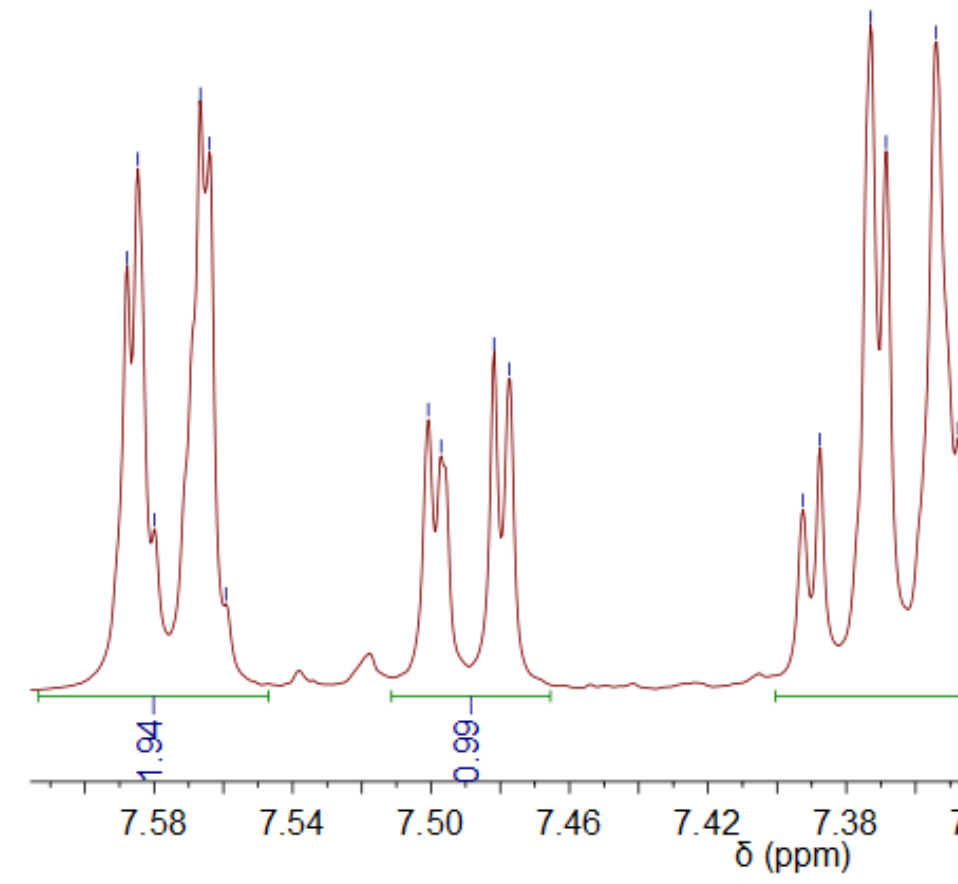

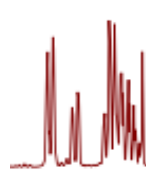

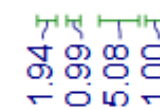

7.5
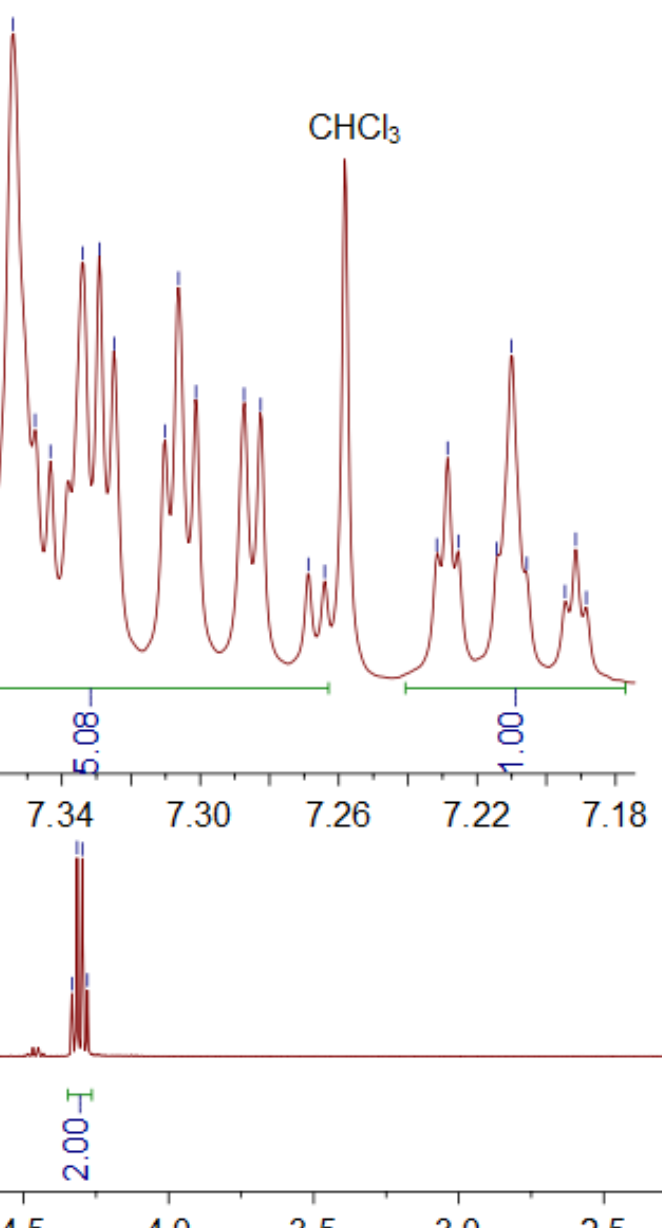

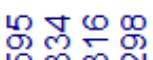

广实

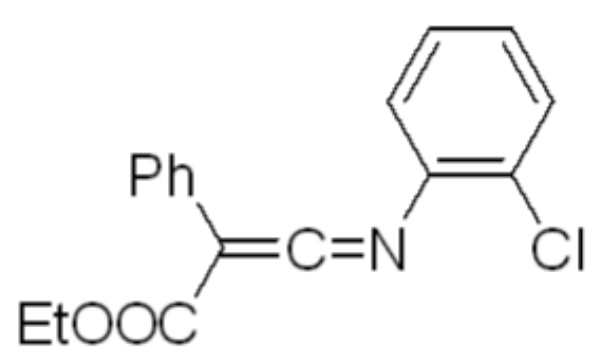

\section{zzy447-157D in $\mathrm{CDCl}_{3}$}

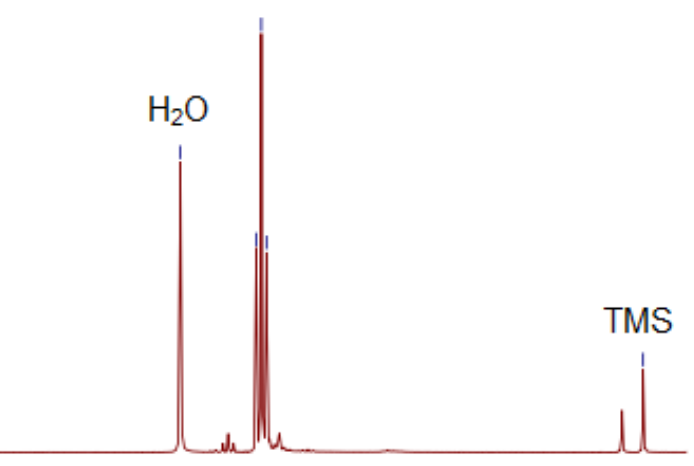

${ }^{\delta}(\mathrm{ppm})$
$\mathbf{2 h}-{ }^{1} \mathrm{H}$ NMR 


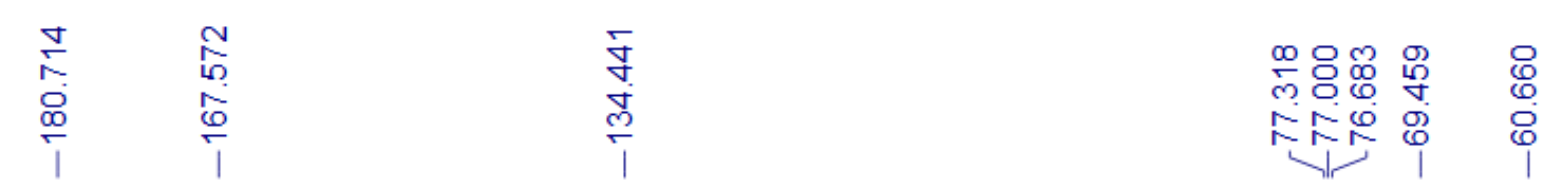

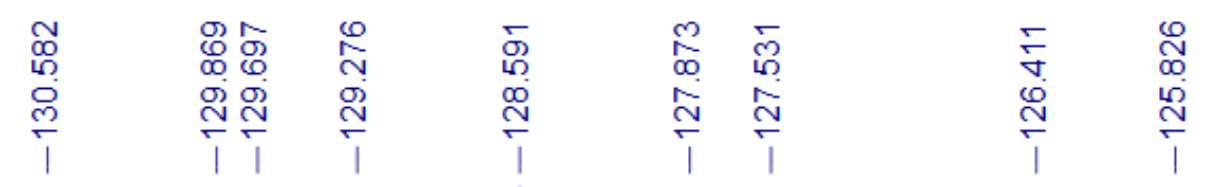

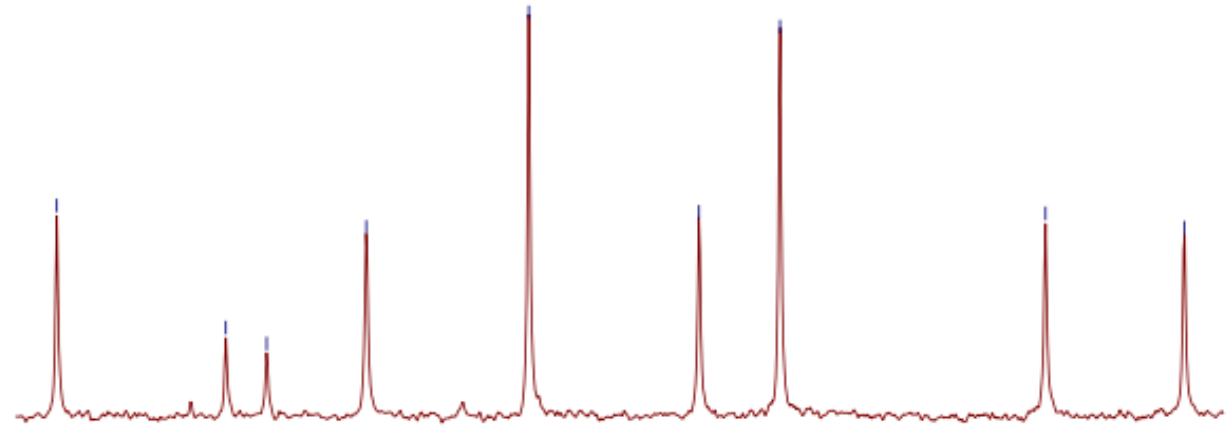

$\mathrm{CDCl}_{3}$

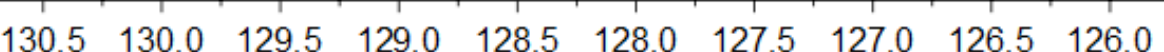
$\delta(\mathrm{ppm})$
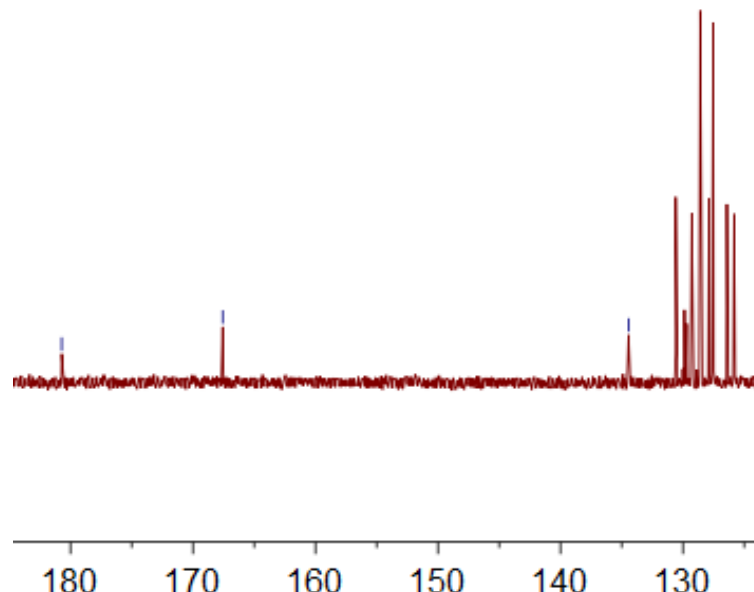

120

110

$100 \underset{\delta(\mathrm{ppm})}{90} 80$

$\mathbf{2 h}-{ }^{13} \mathrm{C}$ NMR 


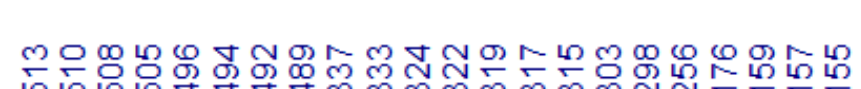

कि की की

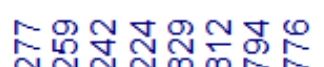

,
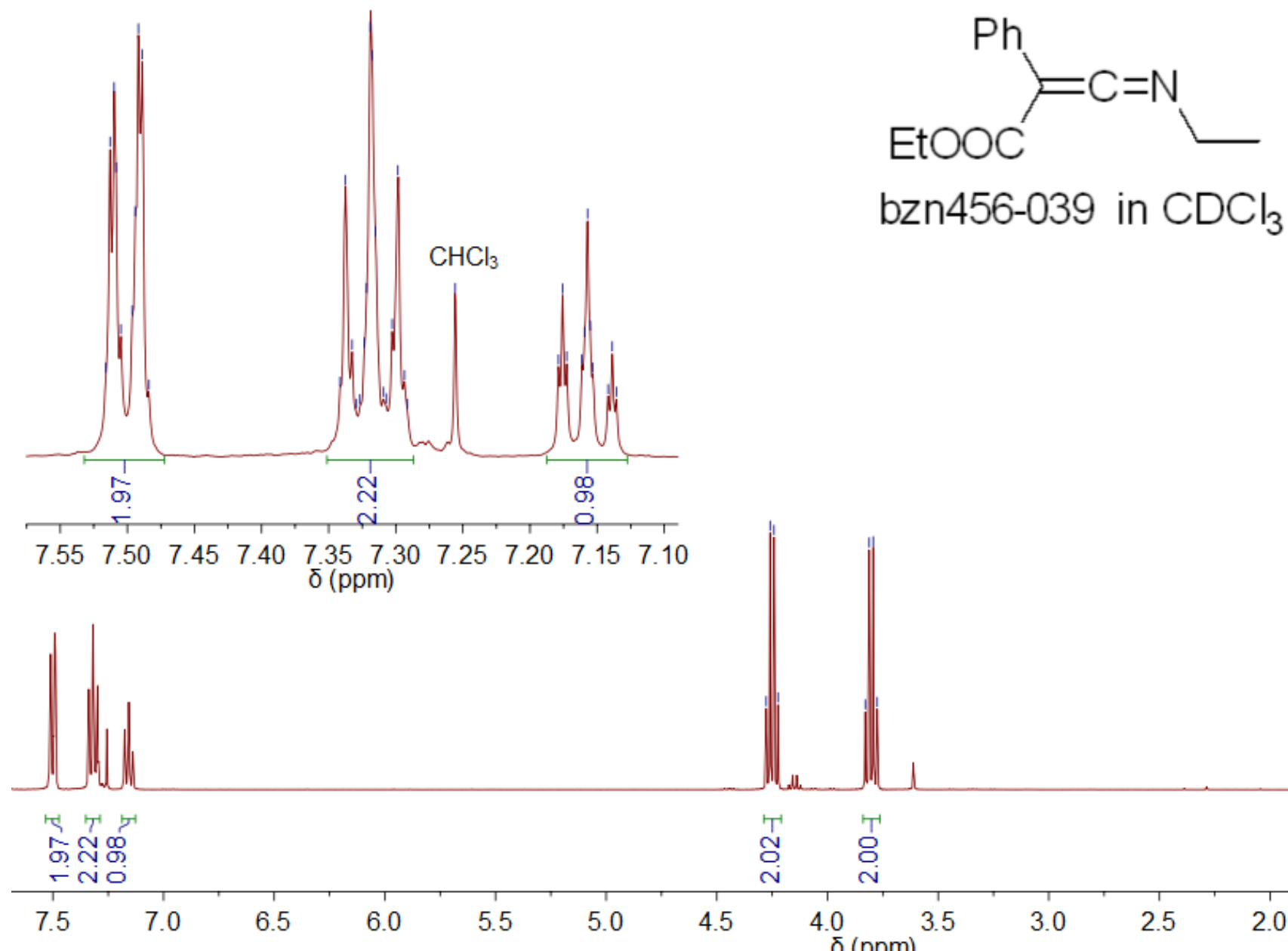

T⿱宀

$\stackrel{\text { T' }}{\mathrm{C}}$

$\delta(\mathrm{ppm})$

$\mathbf{2 i}-{ }^{1} \mathrm{H}$ NMR 

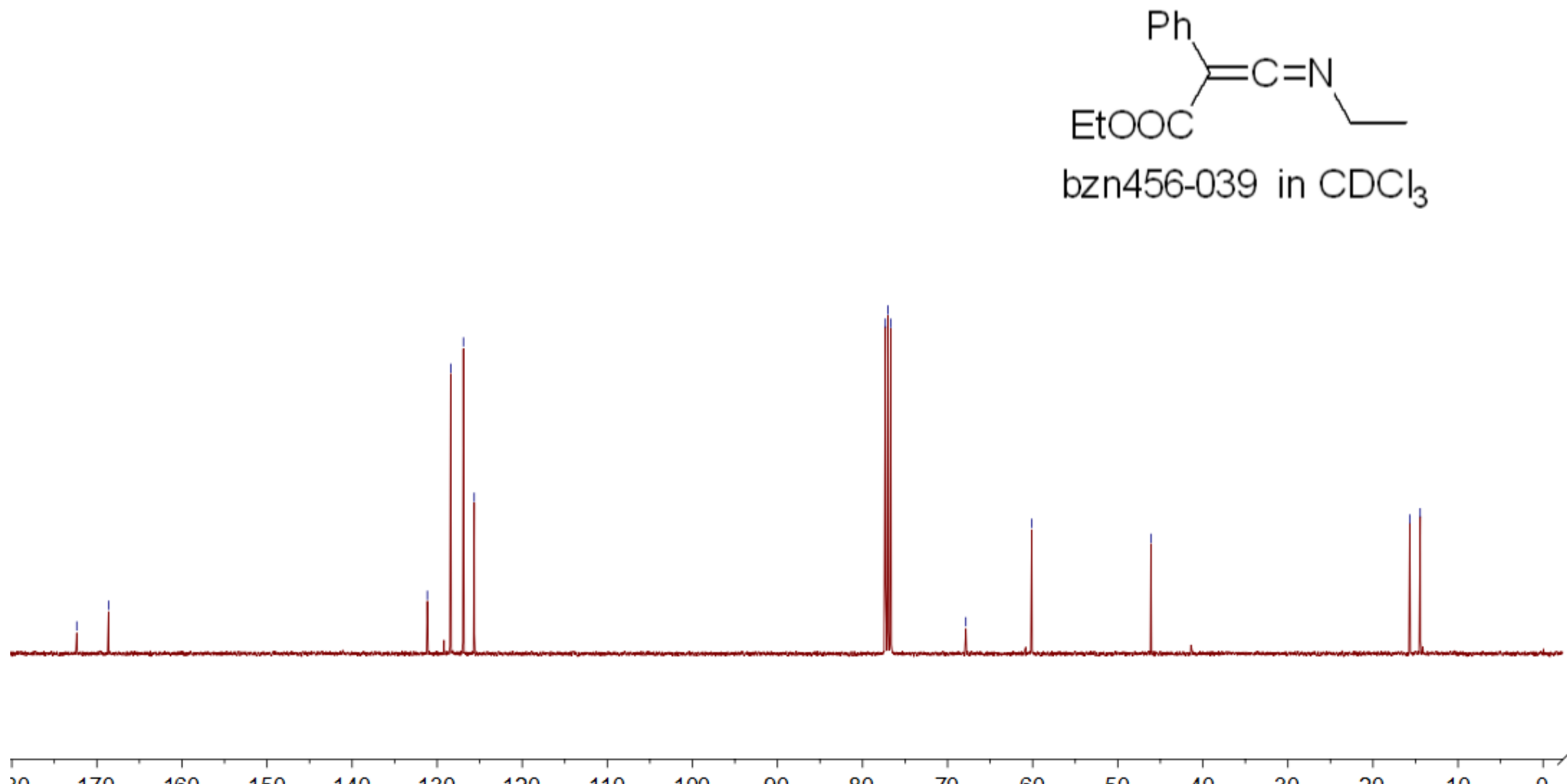

160
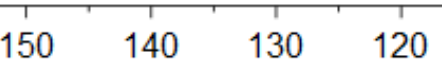
$2 \mathbf{i}-{ }^{13} \mathrm{C} \mathrm{NMR}$ 


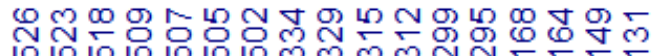

MNMNMNMNMNM

$\Rightarrow$ in

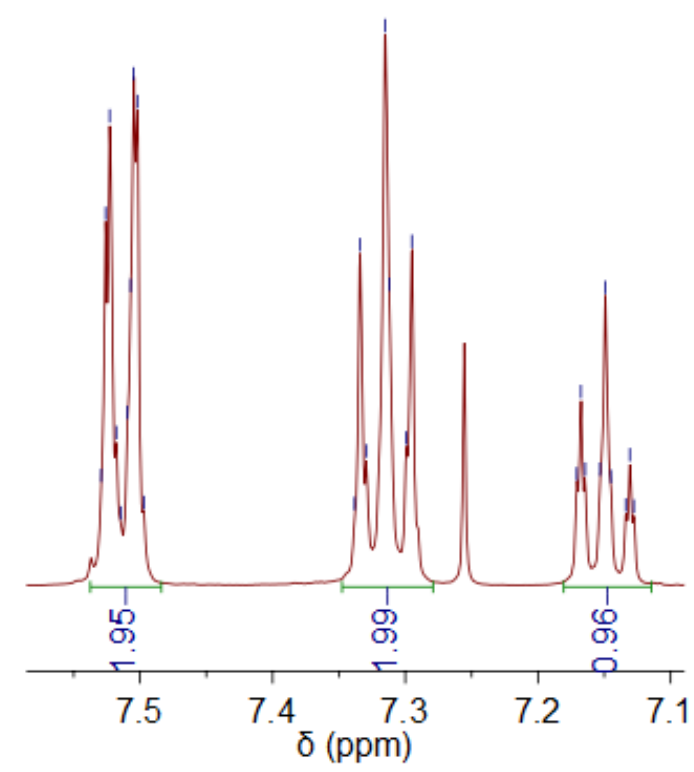

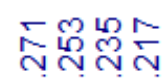

$\int \underset{i}{2}$

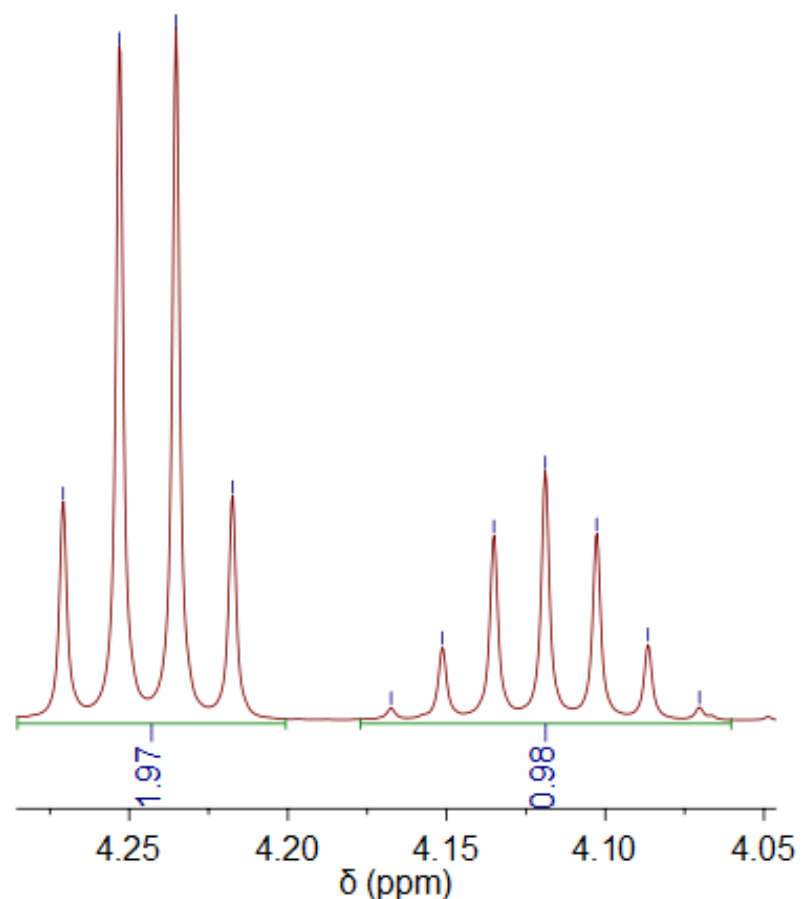

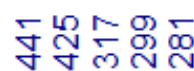

-Ti

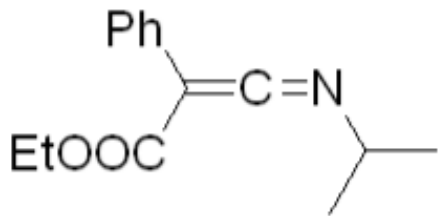

\section{bzn456-040 in $\mathrm{CDCl}_{3}$}

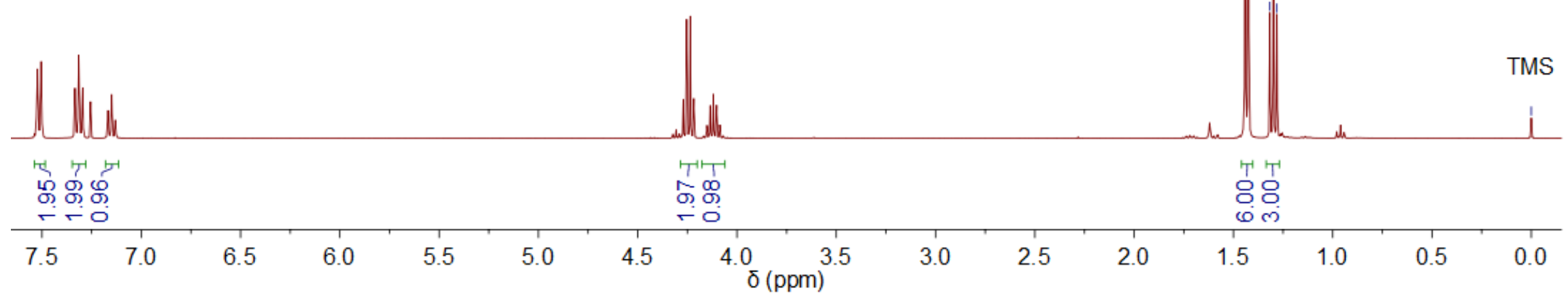

$\mathbf{2 j}-{ }^{1} \mathrm{H}$ NMR 


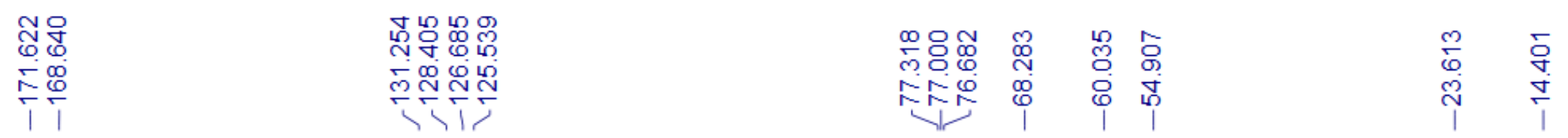

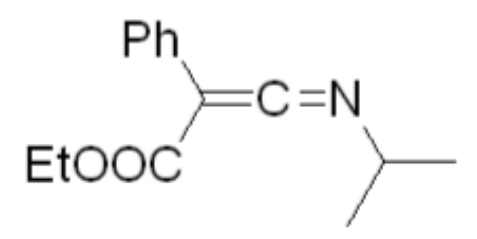

bzn456-040 in $\mathrm{CDCl}_{3}$

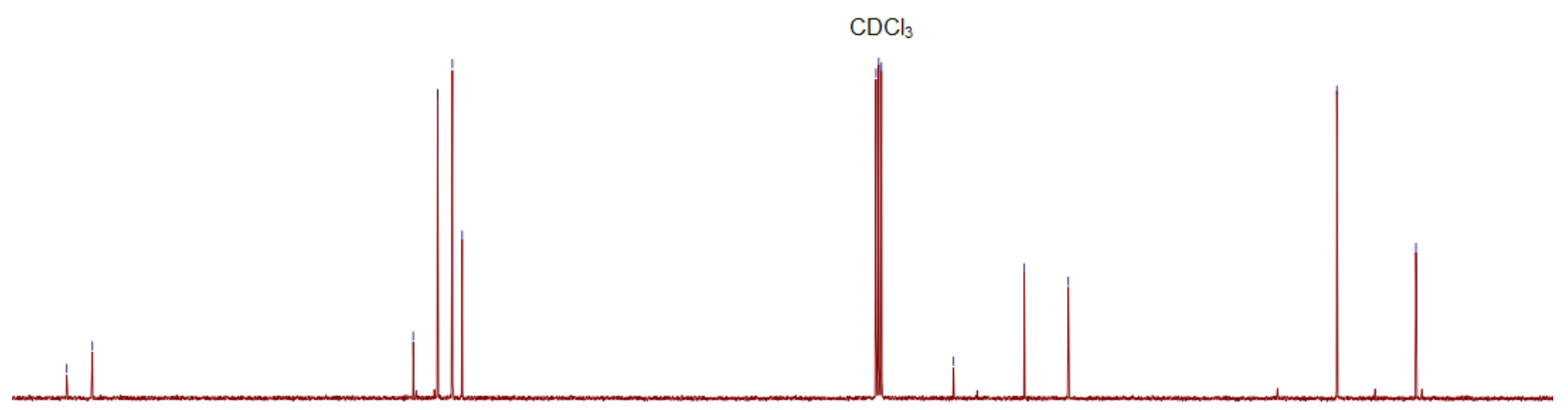

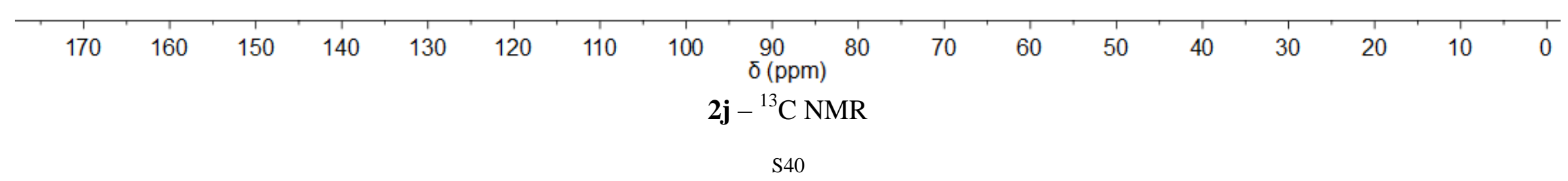




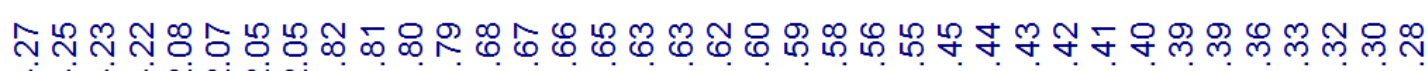
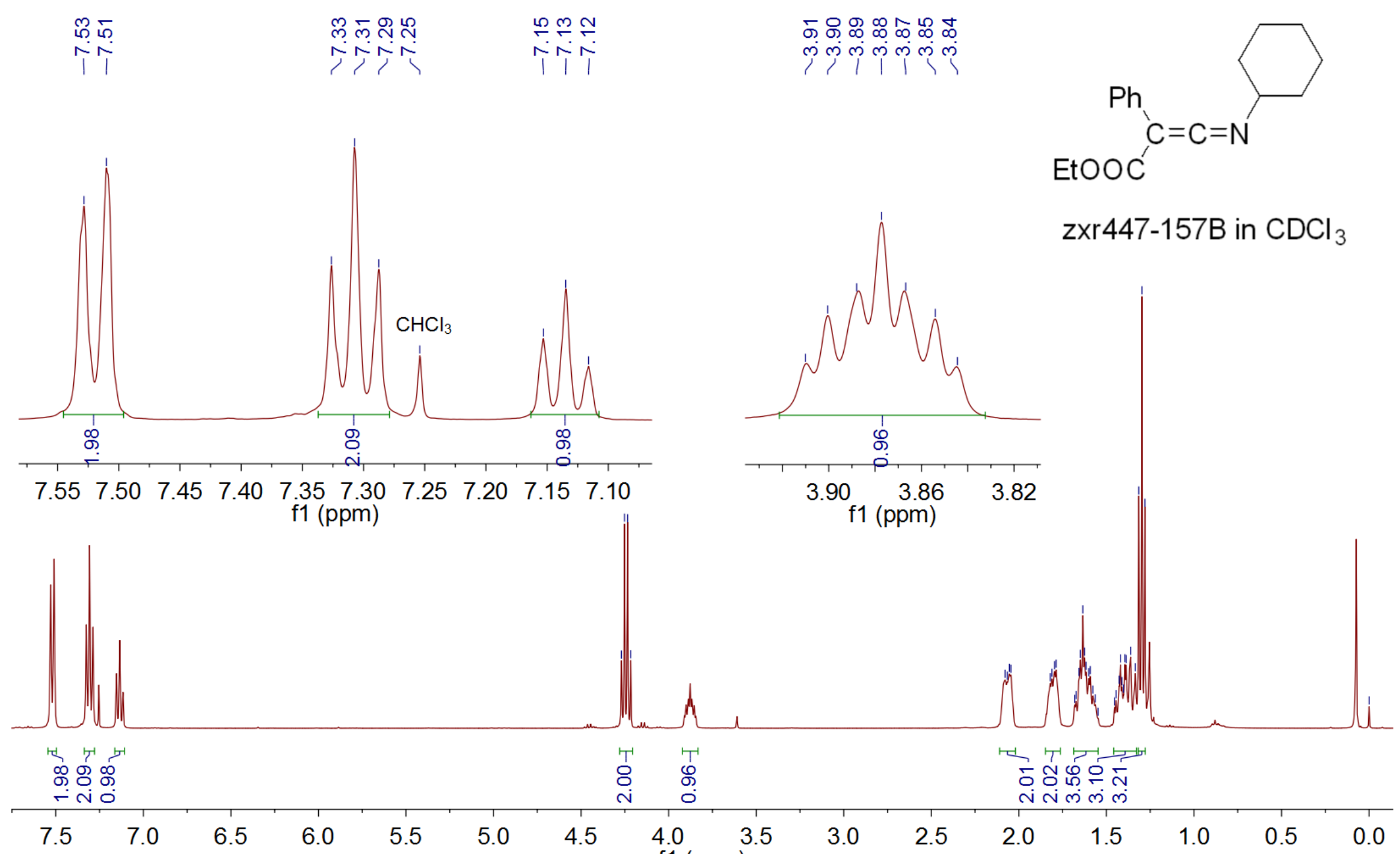

$$
\begin{aligned}
& \text { f1 (ppm) } \\
& \text { 2k- }-{ }^{1} \mathrm{H} \text { NMR }
\end{aligned}
$$




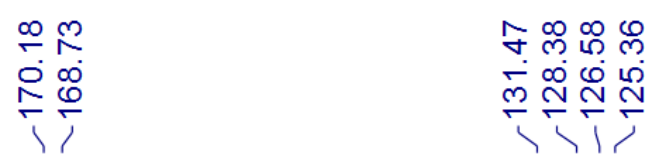

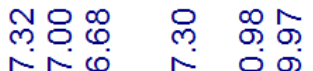

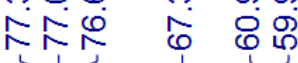

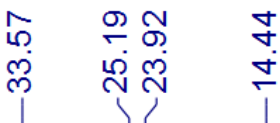

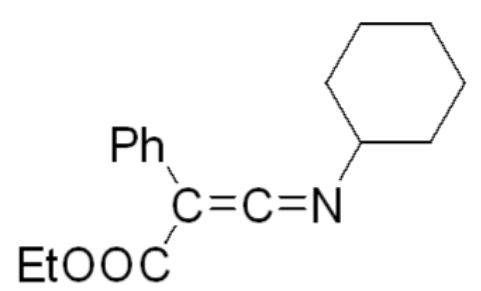

zxr447-157B in $\mathrm{CDCl}_{3}$

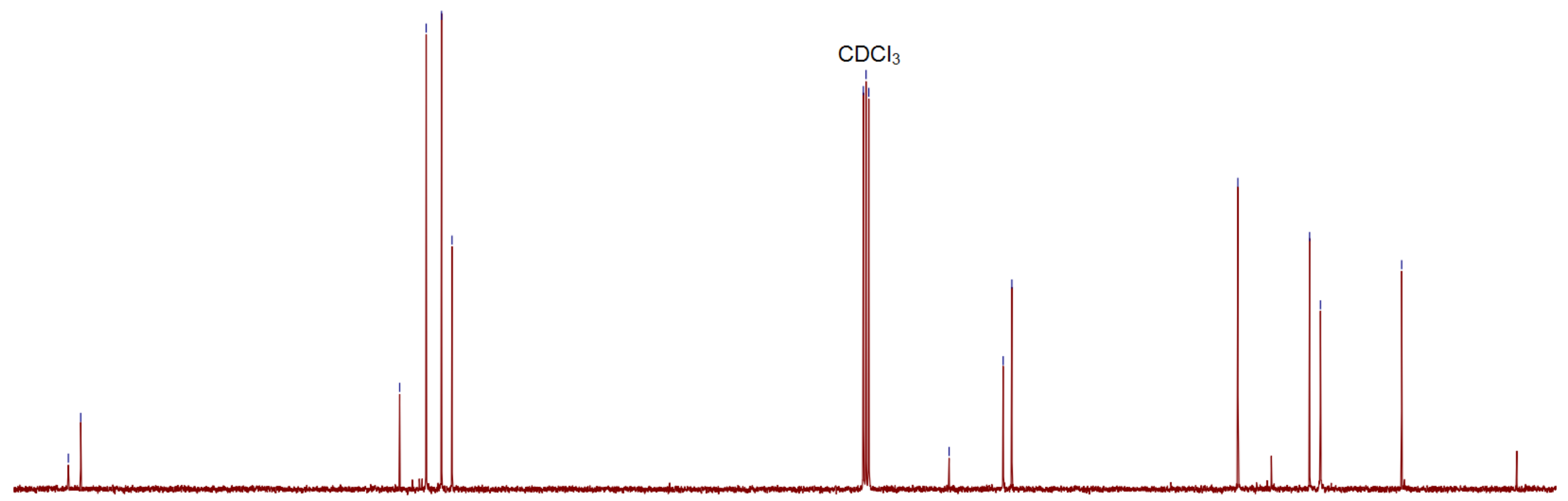

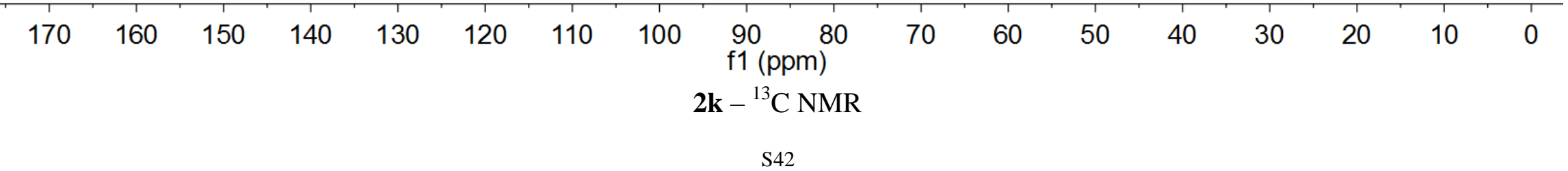




\begin{tabular}{|c|c|c|c|}
\hline 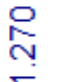 & 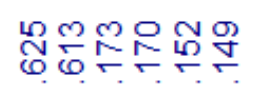 & 呫 号 & 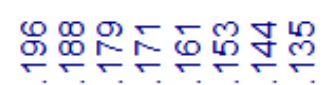 \\
\hline$\check{\digamma}$ & $\underbrace{\infty} \infty \underbrace{\infty}$ & $\underbrace{\circ}$ & 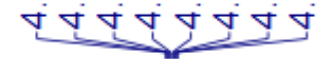 \\
\hline
\end{tabular}

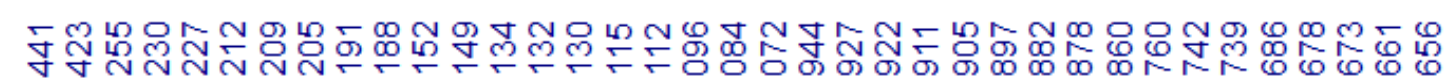

-
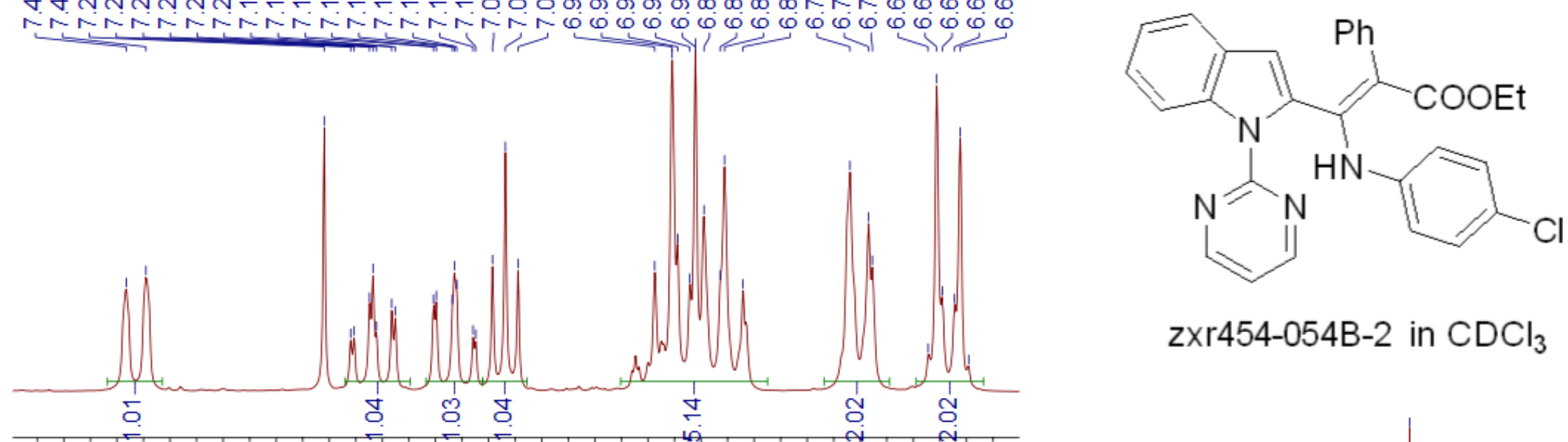

zxr454-054B-2 in $\mathrm{CDCl}_{3}$

7.50 7.45 7.40 7.35 7.30 7.25 7.20 7.157.107.05 7.006.956.906.856.806.756.706.65

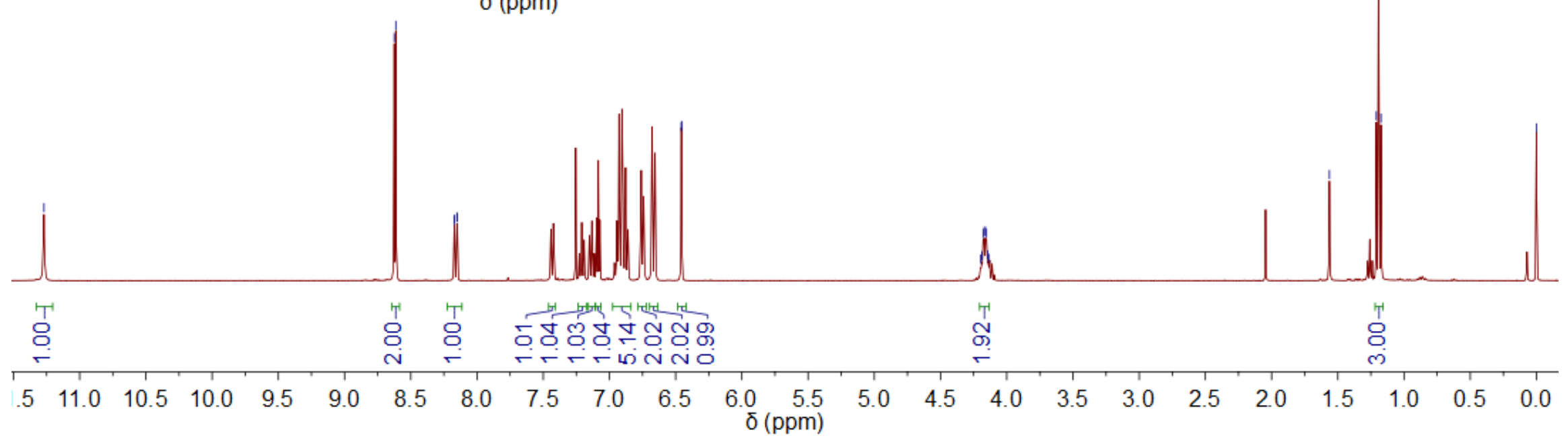

$\mathbf{3 a}-{ }^{1} \mathrm{H}$ NMR 


\begin{tabular}{|c|c|c|c|c|}
\hline 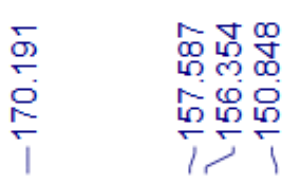 & 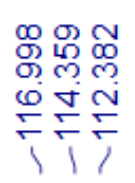 & $\begin{array}{l}\stackrel{g}{N} \\
\stackrel{\sigma}{\sigma}\end{array}$ & 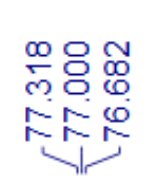 & $\begin{array}{c}5 \\
\infty \\
0 \\
0 \\
0 \\
1 \\
1\end{array}$ \\
\hline
\end{tabular}
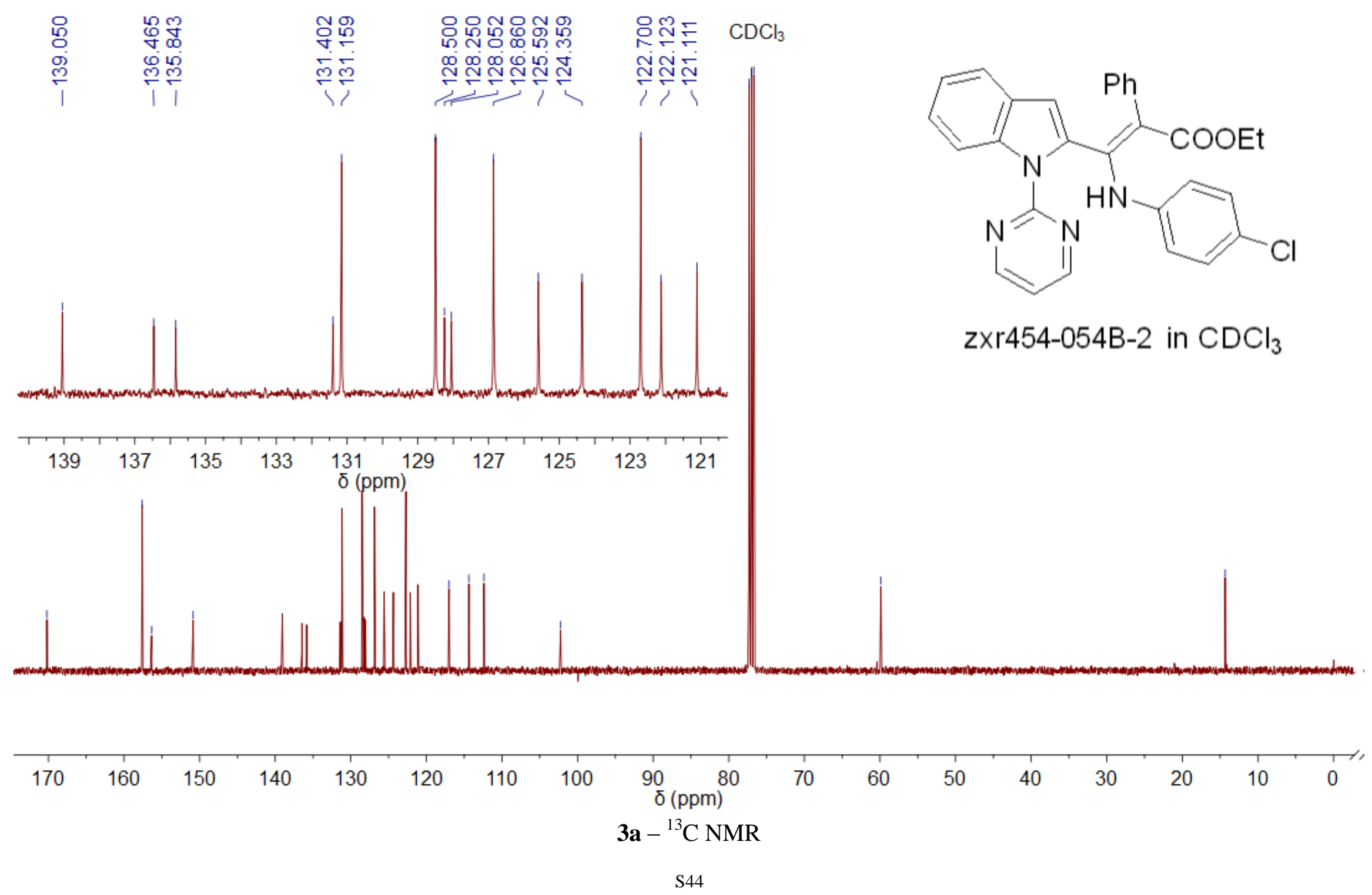


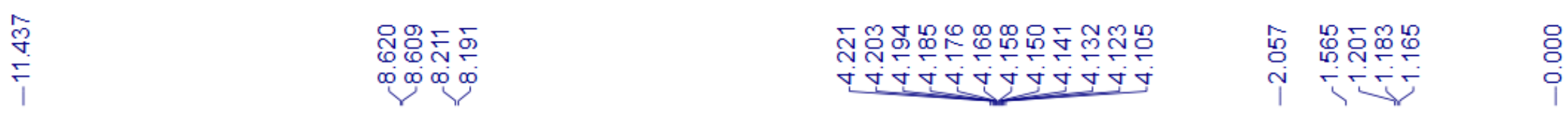

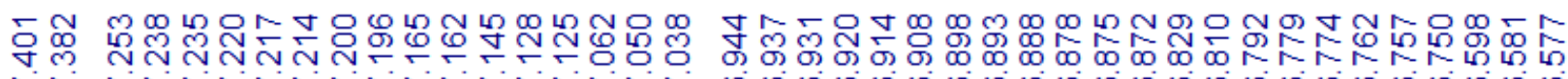

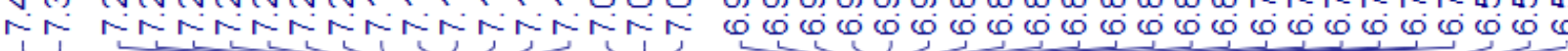
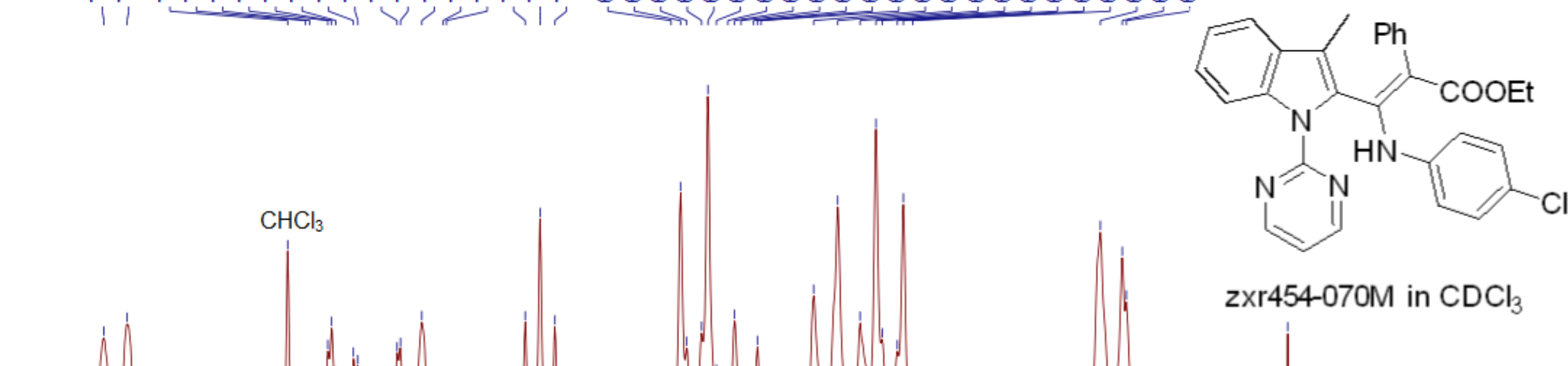

8

ช.

$\begin{array}{llllllllllllllllllll}7.45 & 7.40 & 7.35 & 7.30 & 7.25 & 7.20 & 7.15 & 7.10 & 7.05 & 7.00 & 6.95 & 6.90 & 6.85 & 6.80 & 6.75 & 6.70 & 6.65 & 6.60 & 6.55 & 6.50\end{array}$ $\bar{\delta}(\mathrm{ppm})$

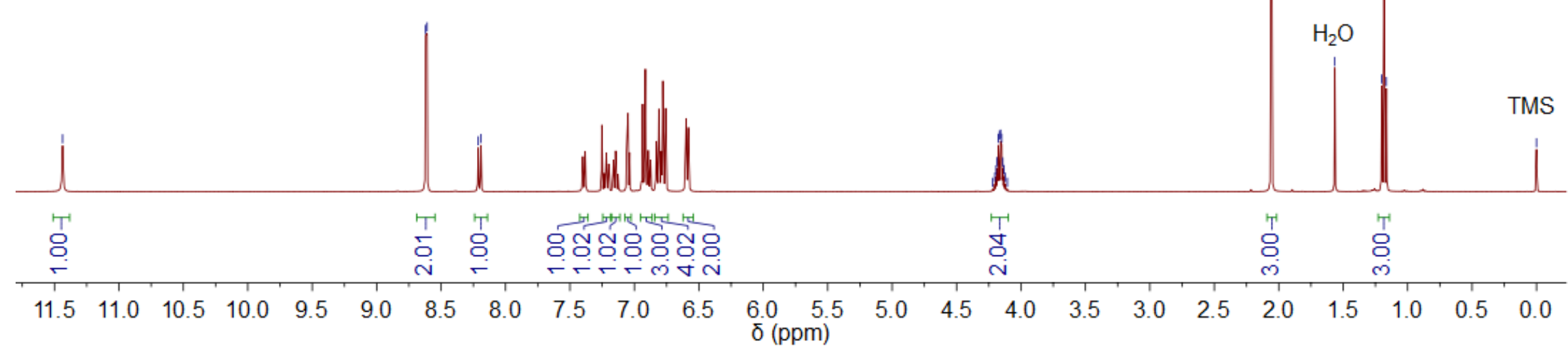

$\mathbf{3 b}-{ }^{1} \mathrm{H}$ NMR 

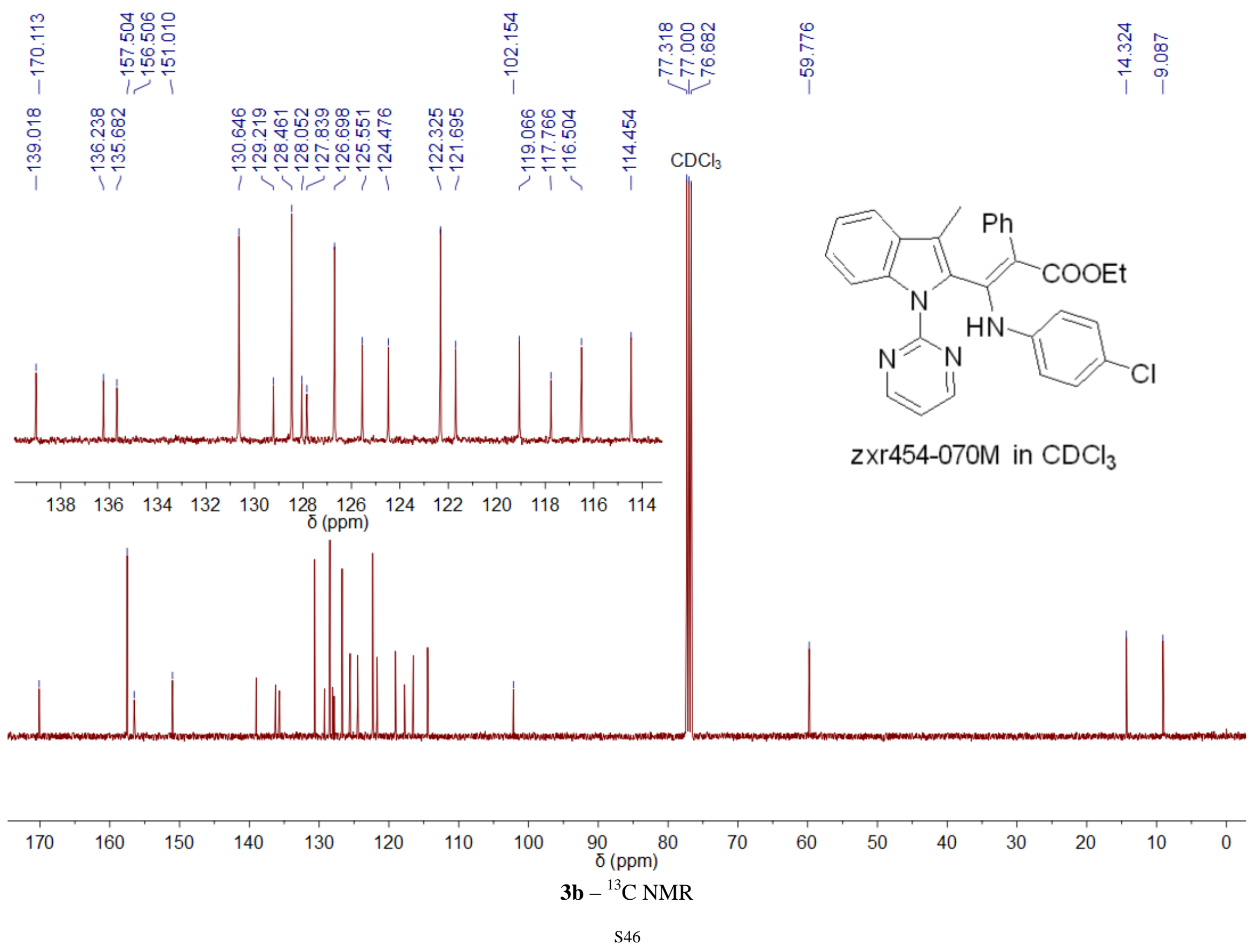


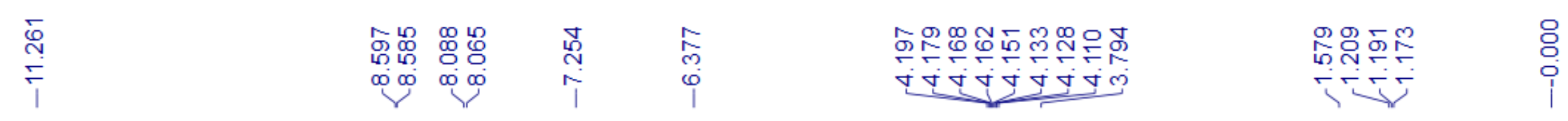

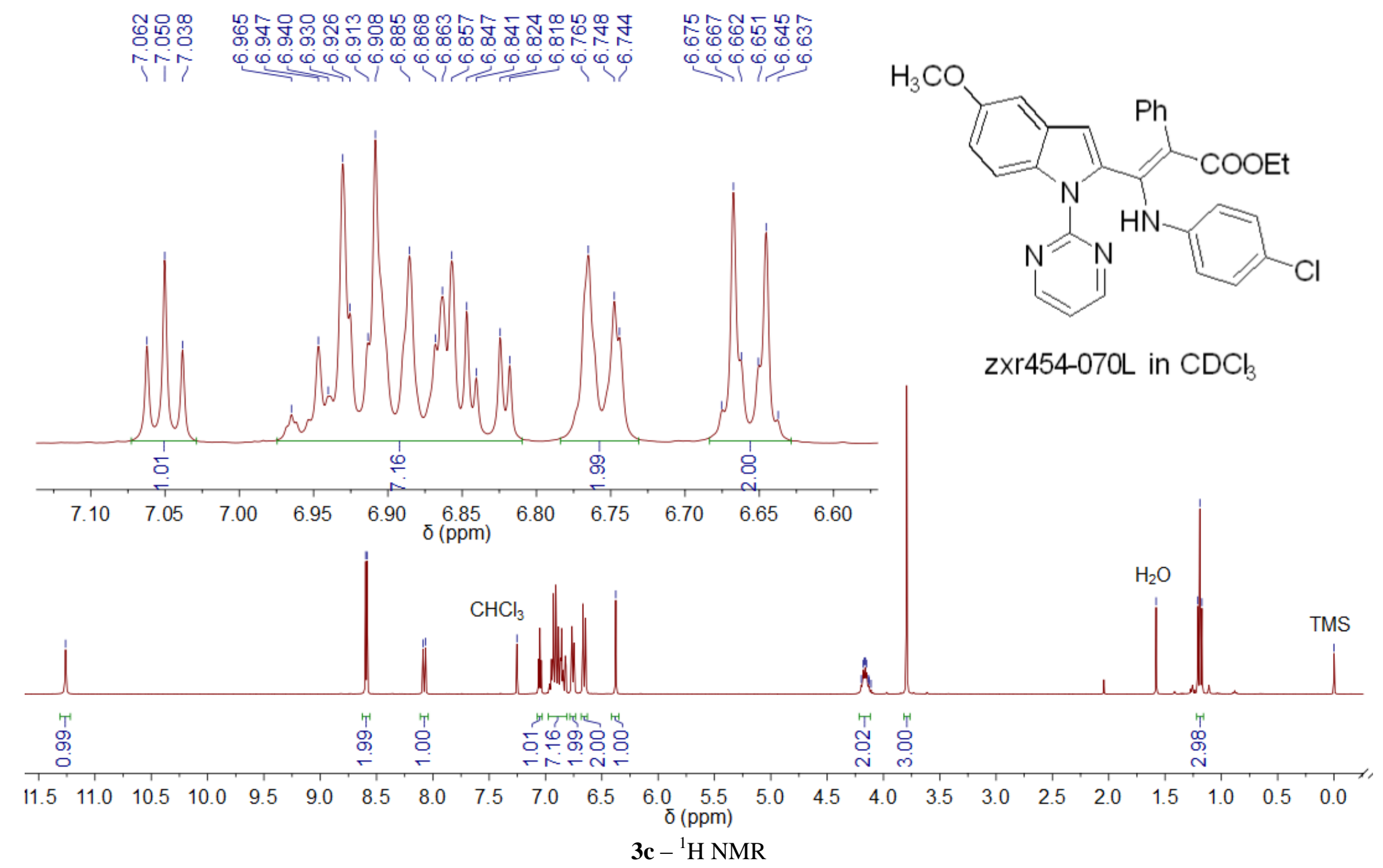




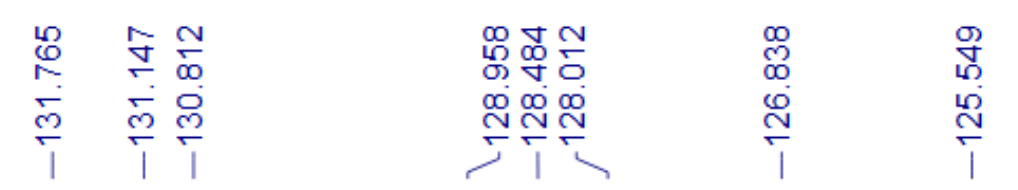

$\mathrm{CDCl}_{3}$

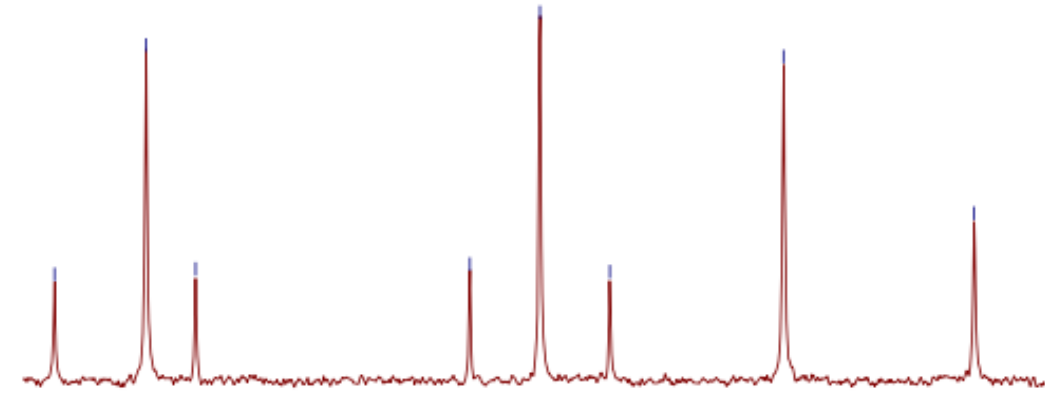

$\mathrm{H}_{3} \mathrm{CO}$

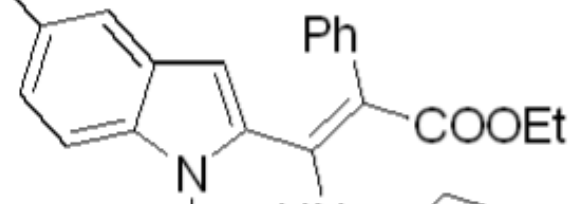<smiles>Clc1ccc(Nc2ncccn2)cc1</smiles>

zxr454-070L in $\mathrm{CDCl}_{3}$
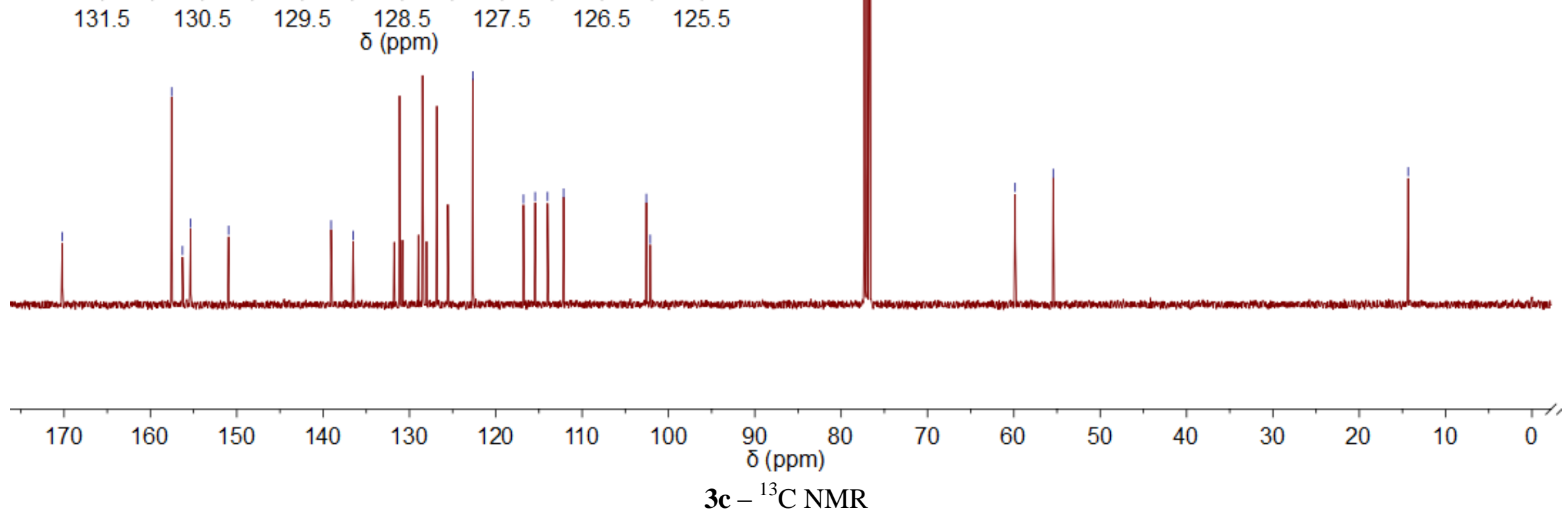


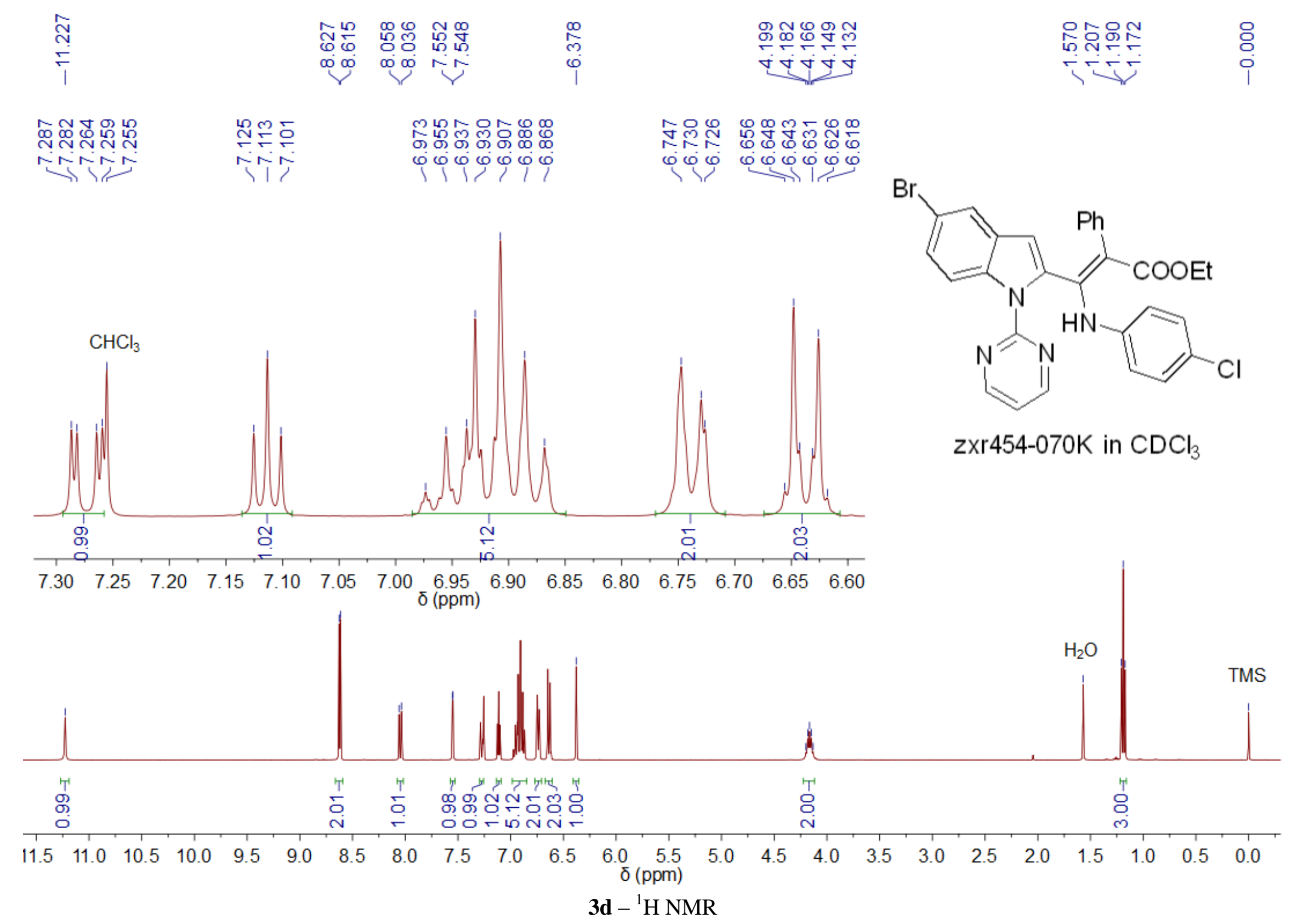



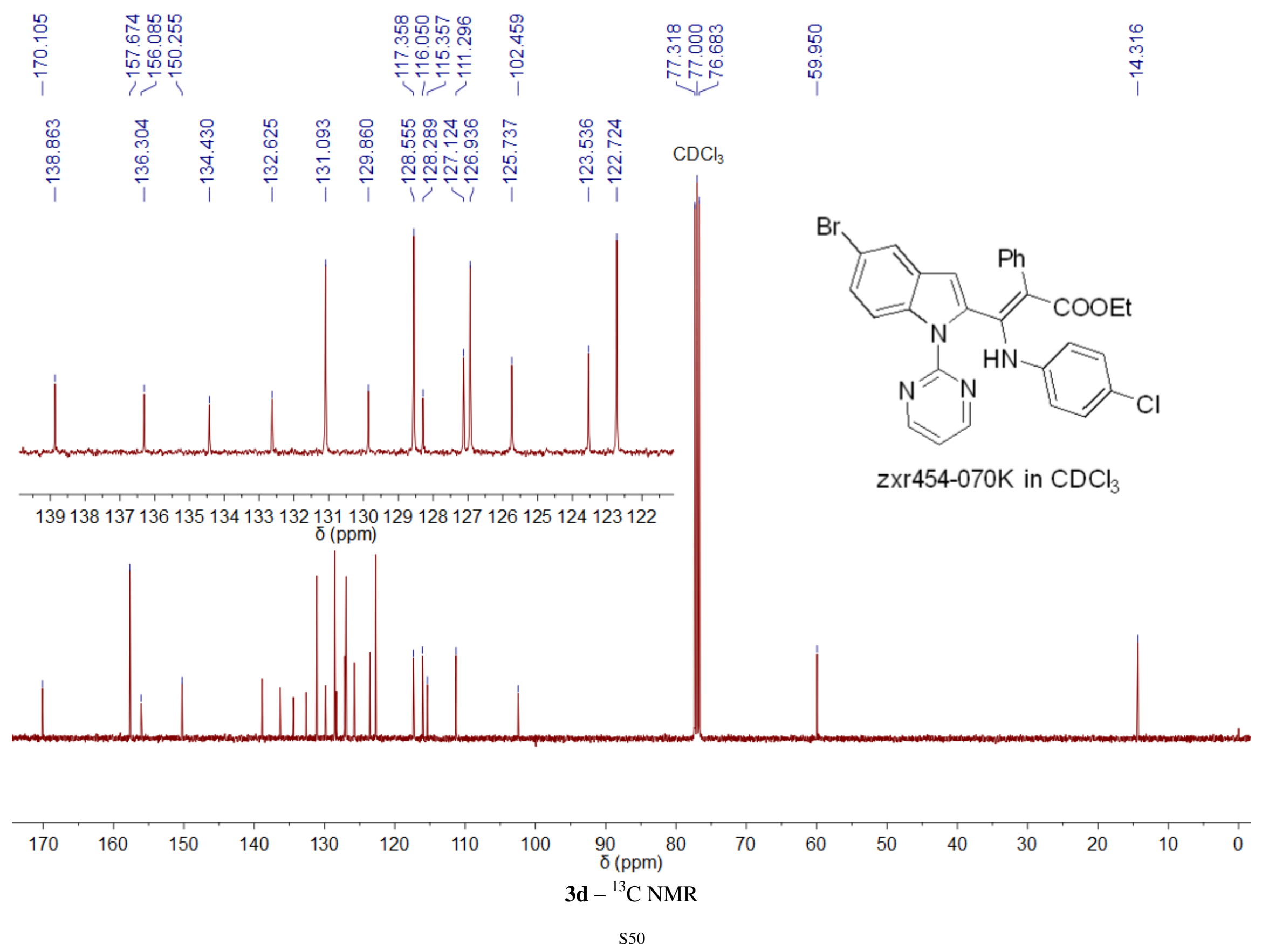


管

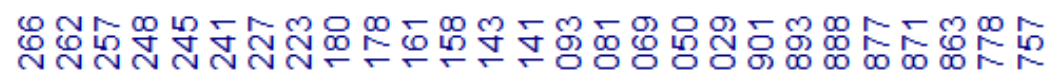

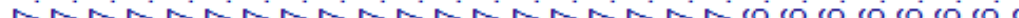
(1)

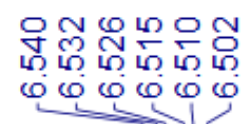
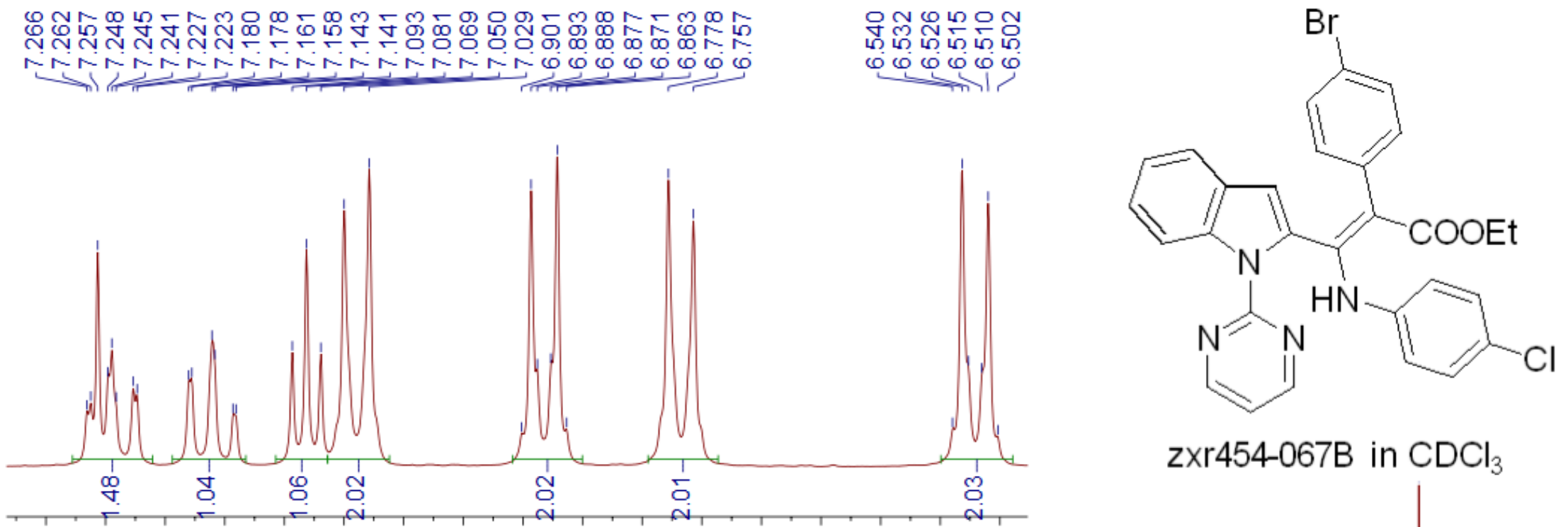
zxr454-067B in $\mathrm{CDCl}_{3}$

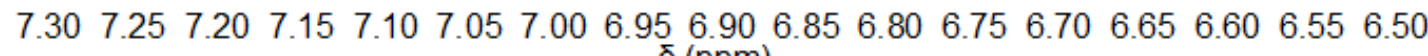
$\bar{\delta}(\mathrm{ppm})$

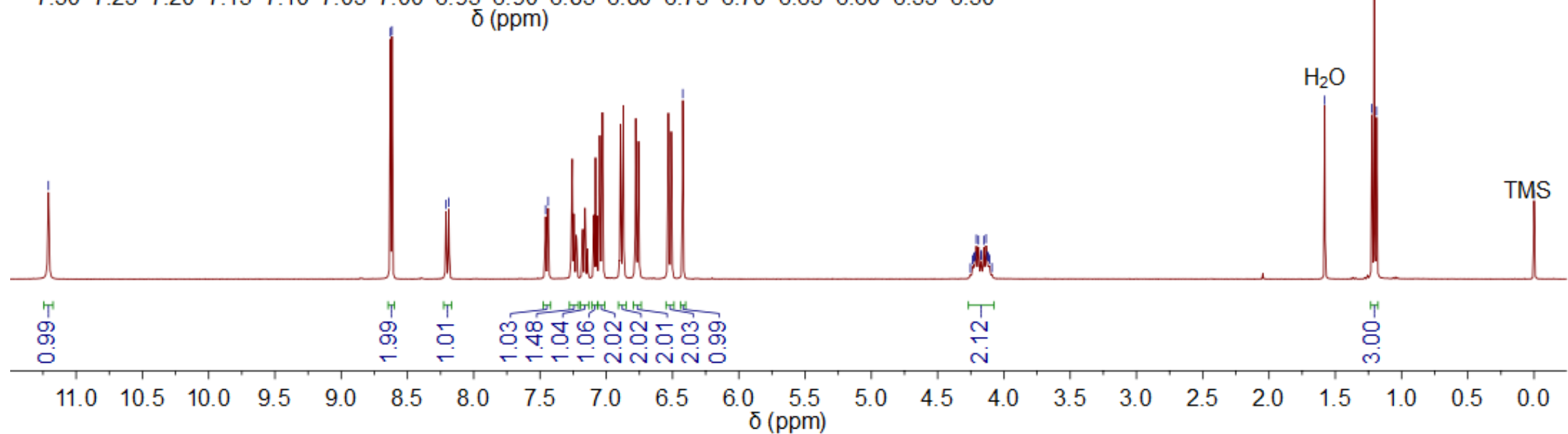
3e $-{ }^{1} \mathrm{H}$ NMR 

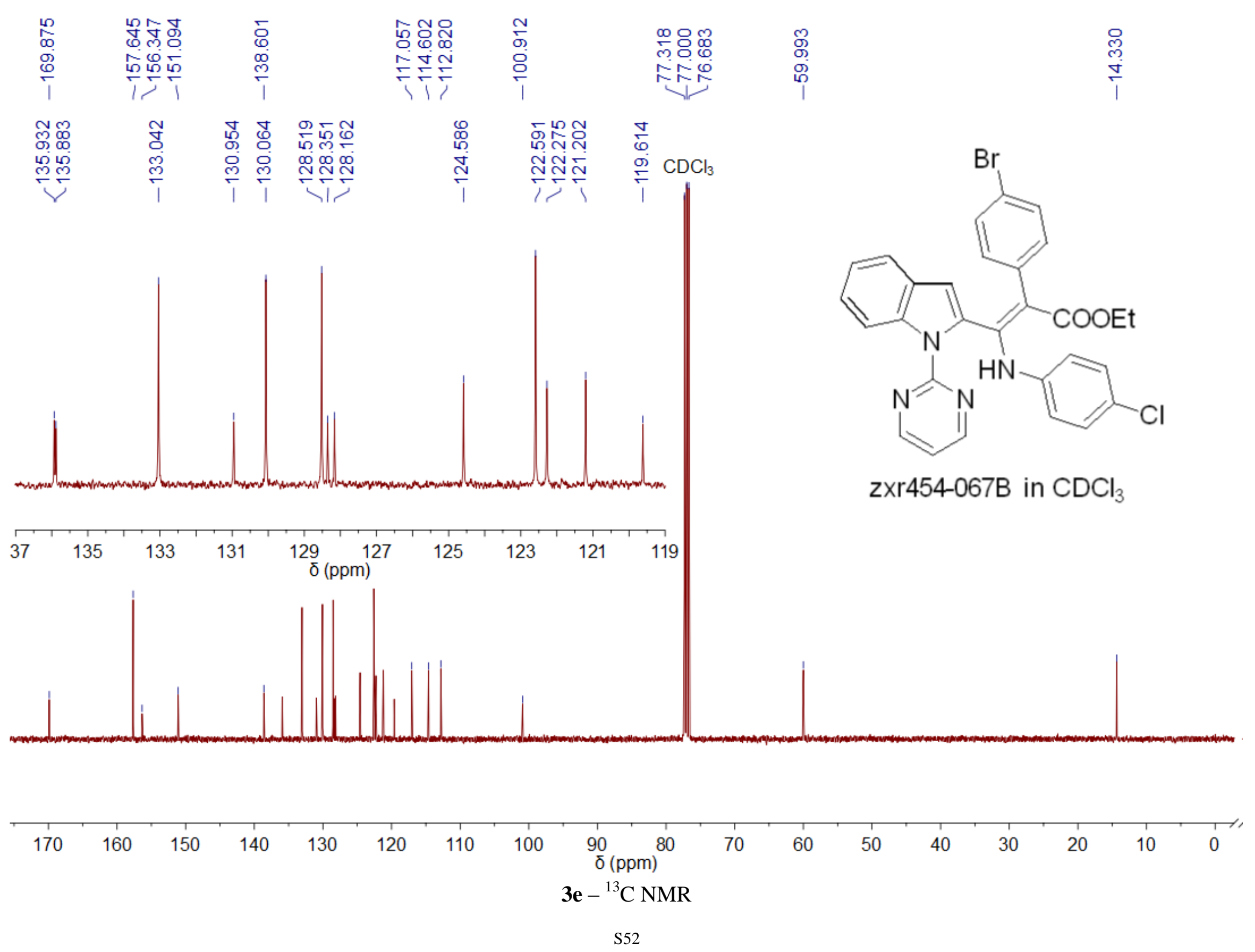

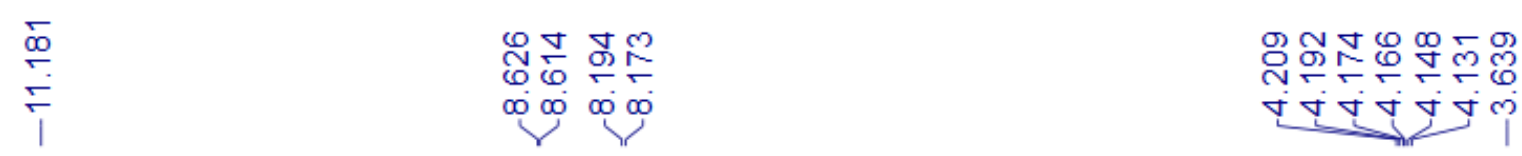

กิำกำ

o

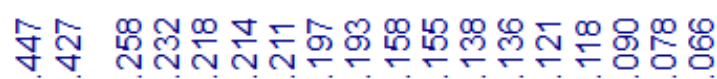

两

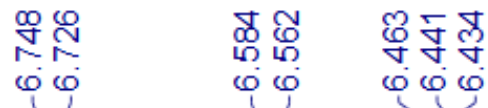

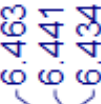

它

$\Rightarrow 2$

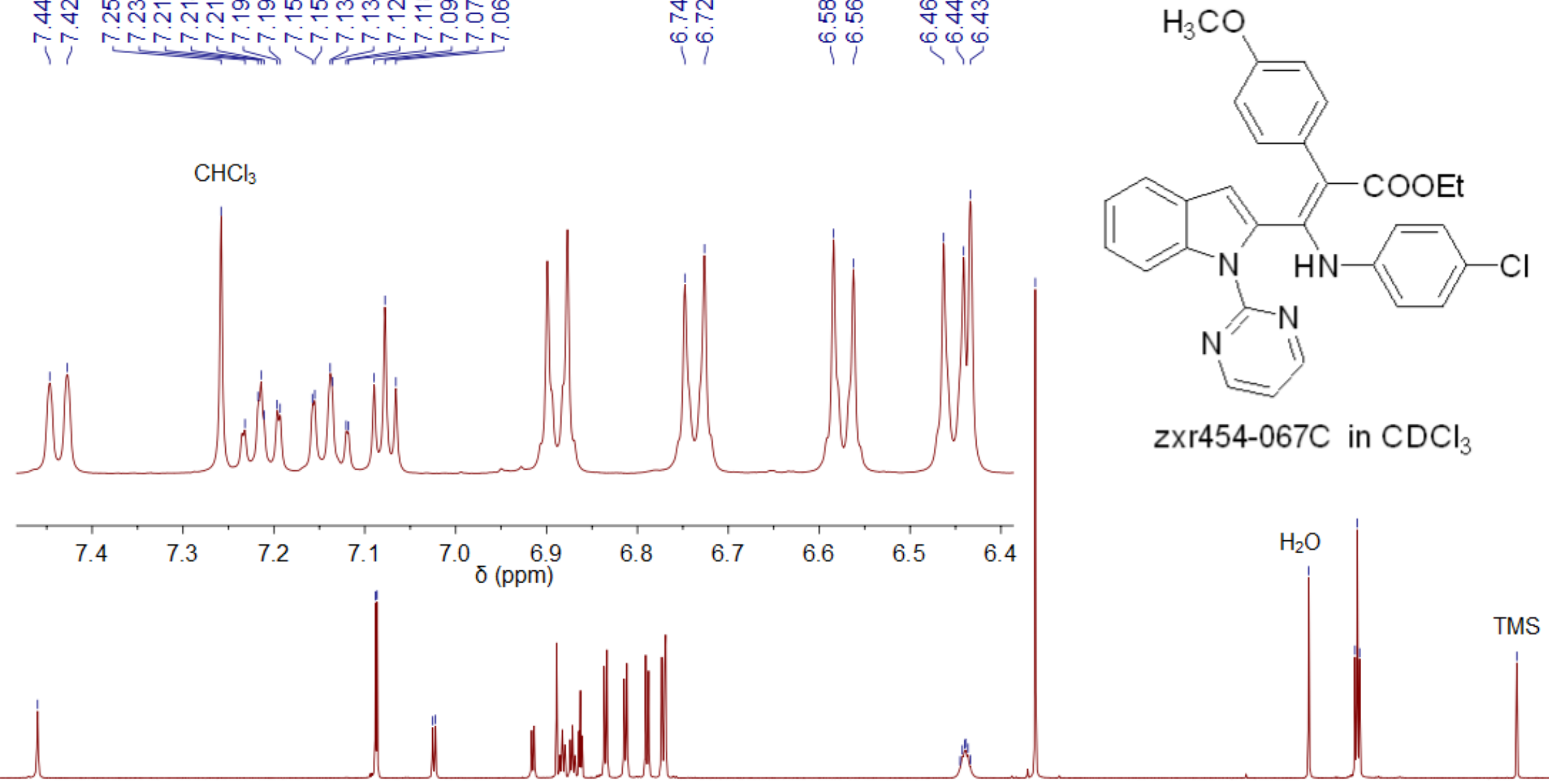

zxr454-067C in $\mathrm{CDCl}_{3}$

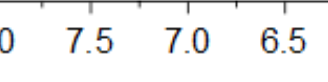

0.5
$\delta(p p m)$

$\begin{array}{llll}4.5 & 4.0 & 3.5 & 3.0\end{array}$

2.5

$2.0 \quad 1.5$

$\begin{array}{lll}1.0 & 0.5 & 0.0\end{array}$

3f $-{ }^{1} \mathrm{H}$ NMR 


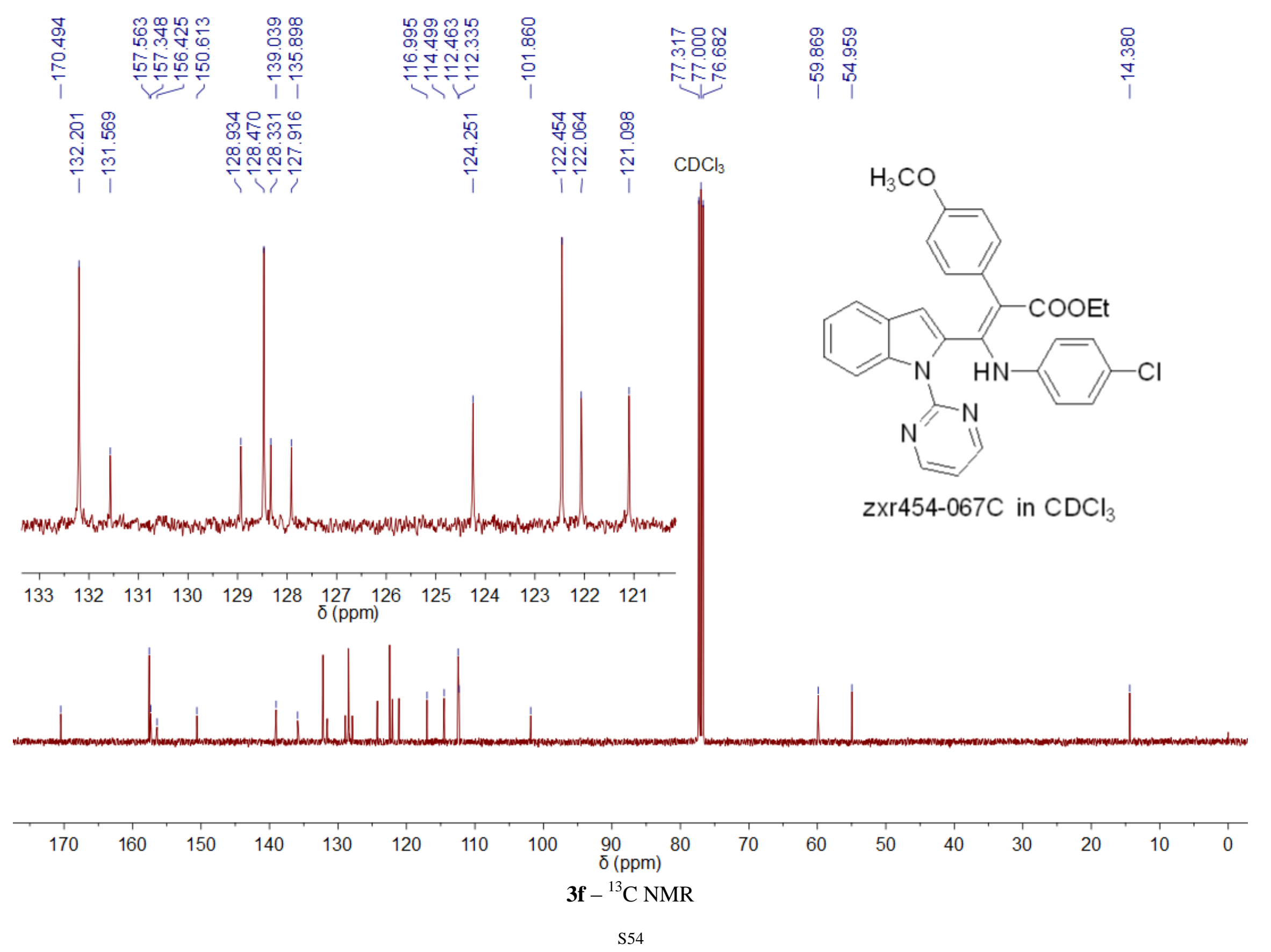




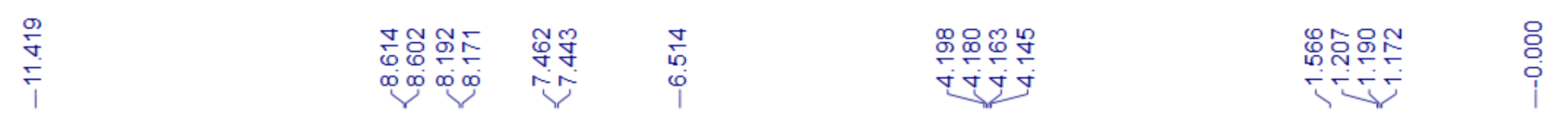

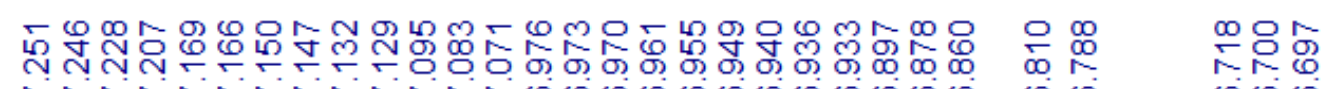

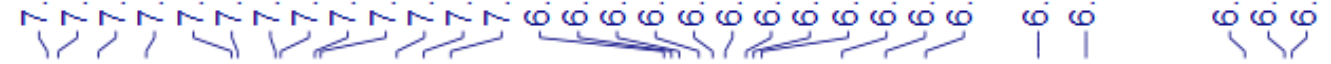
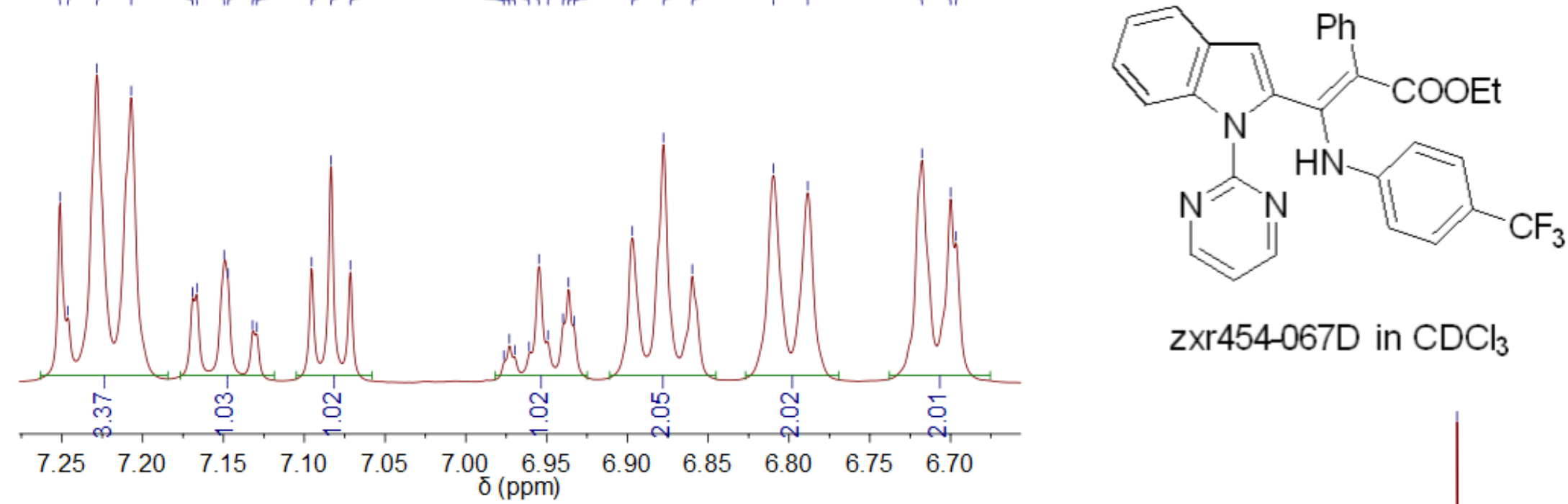

\section{zxr454-067D in $\mathrm{CDCl}_{3}$}

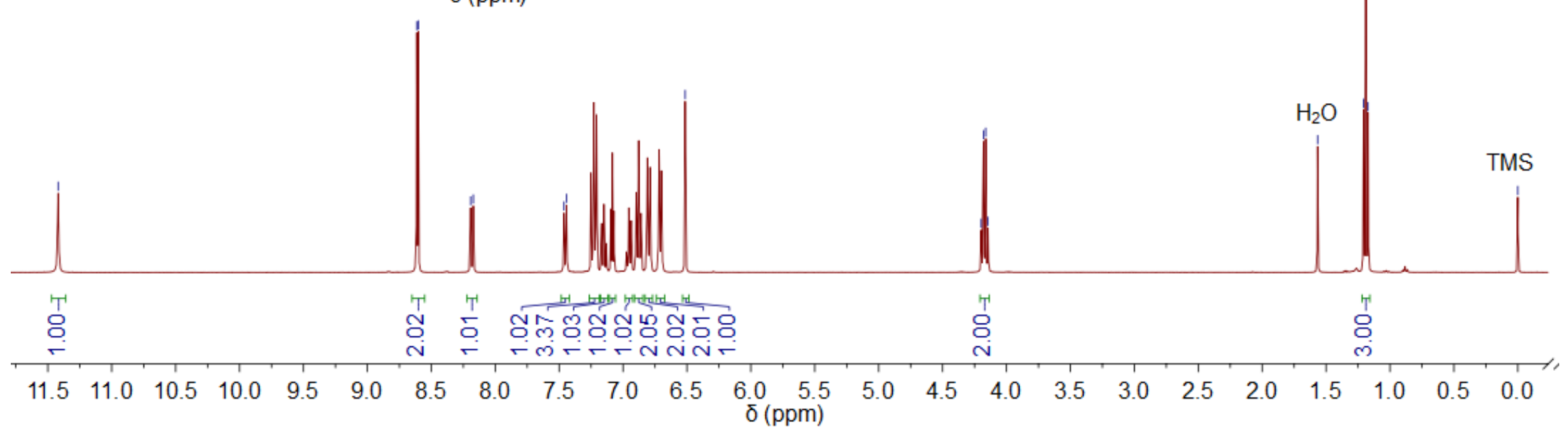

$3 g-{ }^{1} \mathrm{H}$ NMR 


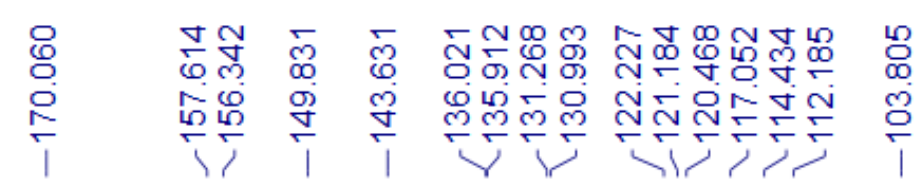

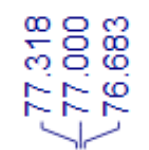

0
0
0
0
0
1
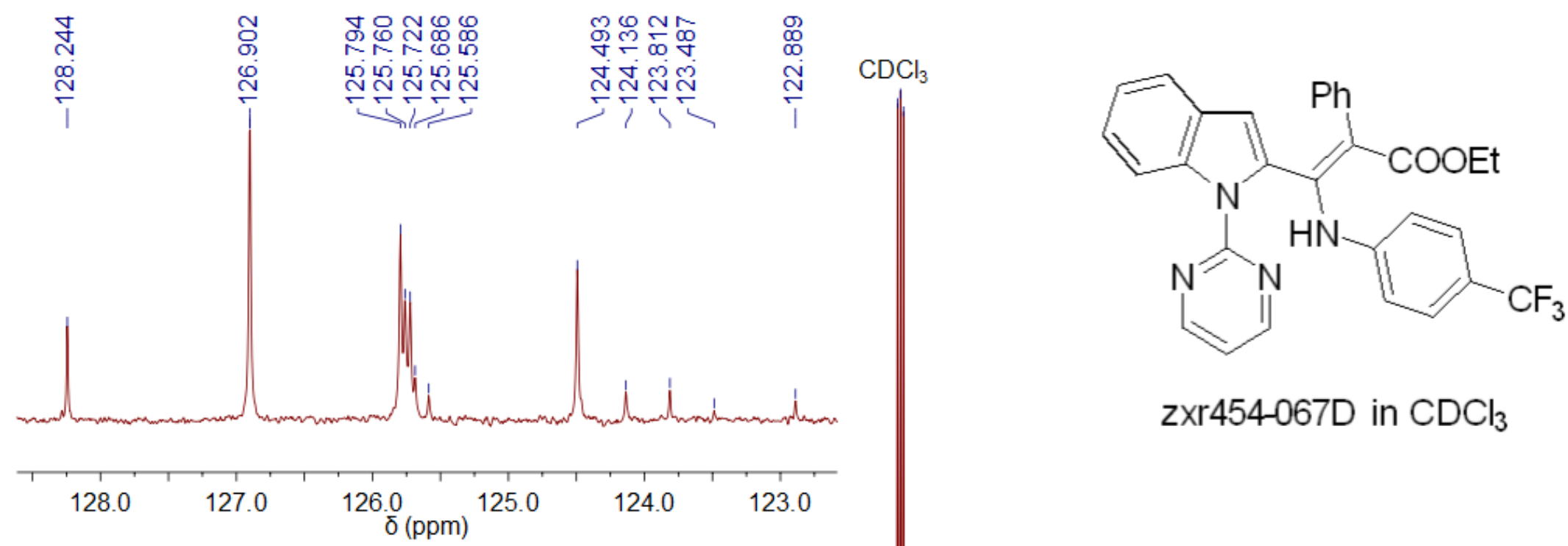

zxr454-067D in $\mathrm{CDCl}_{3}$
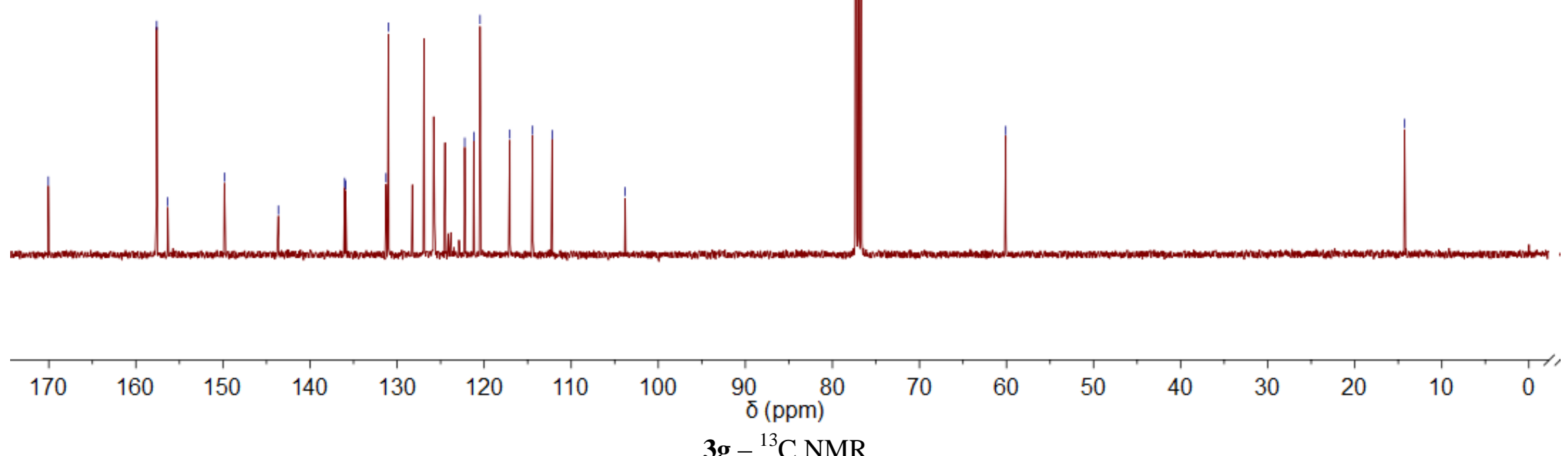


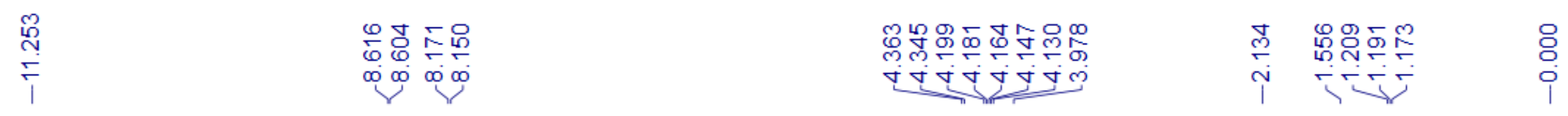

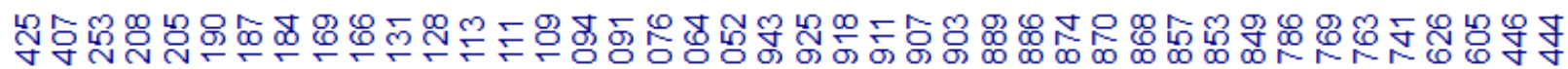

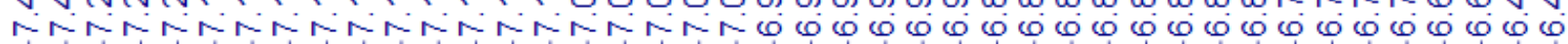

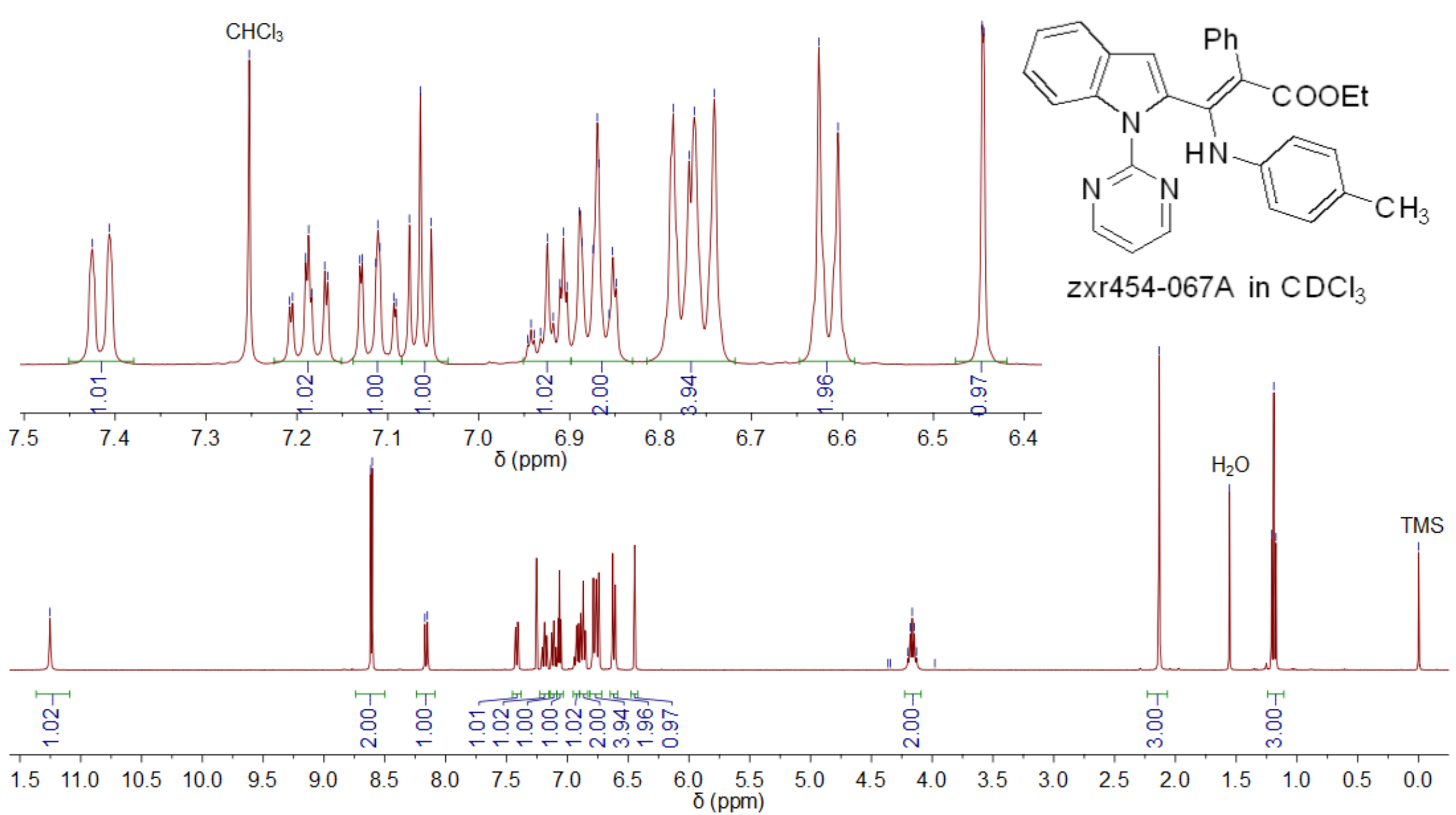

3h $-{ }^{1} \mathrm{H}$ NMR 


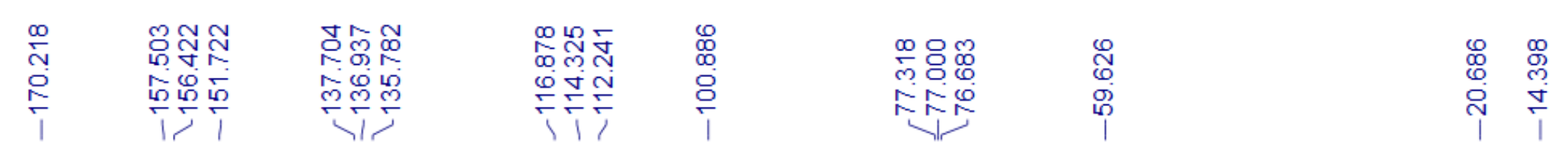
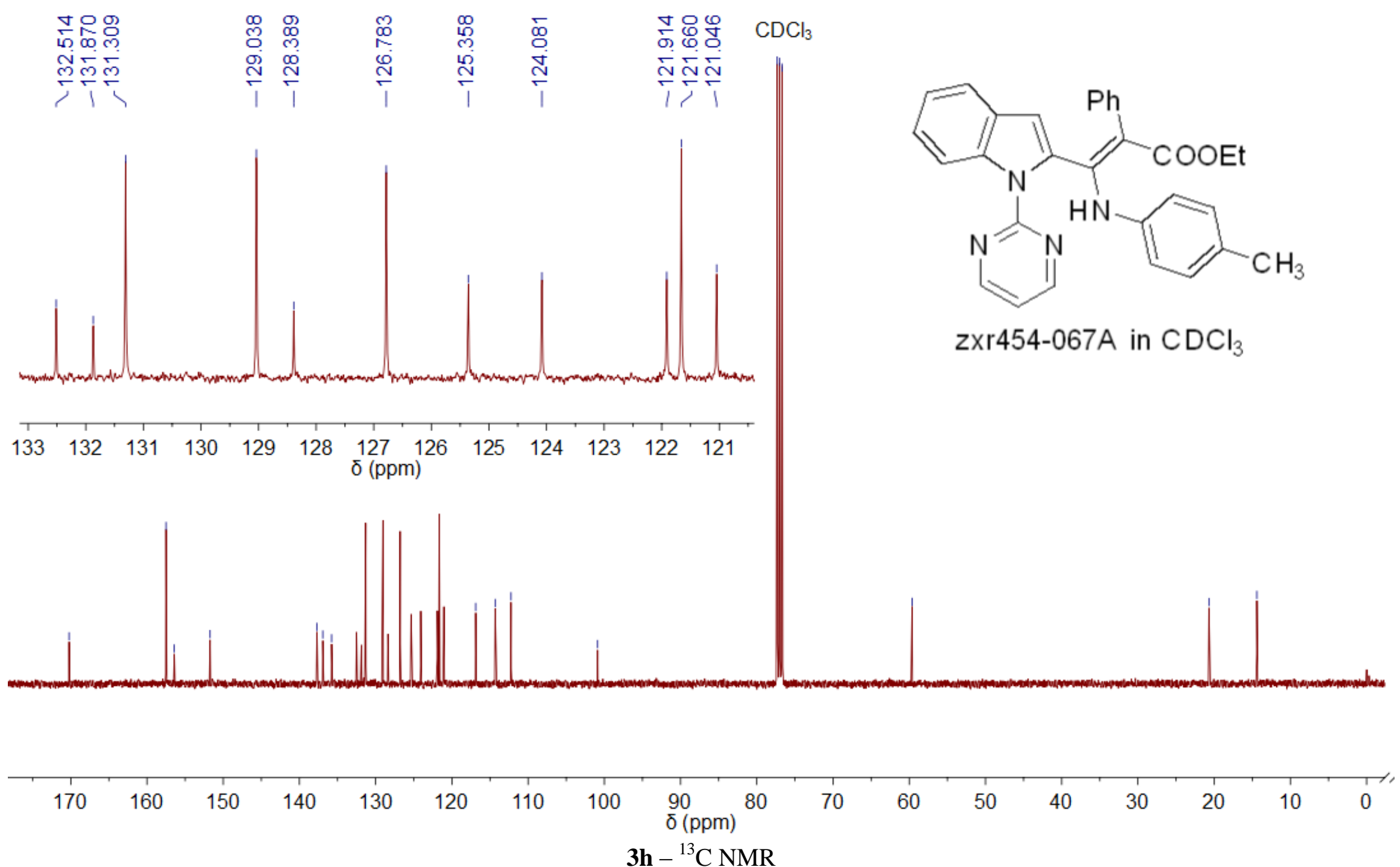


\begin{tabular}{|c|c|c|}
\hline 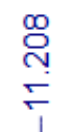 & 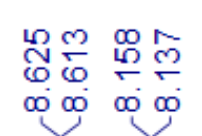 & 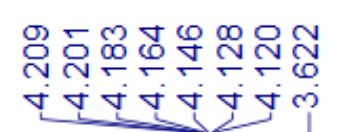 \\
\hline
\end{tabular}

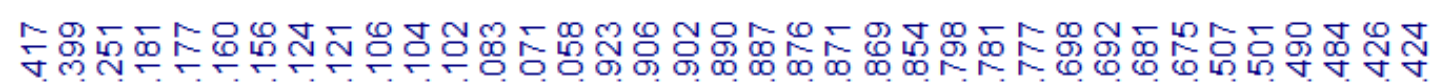

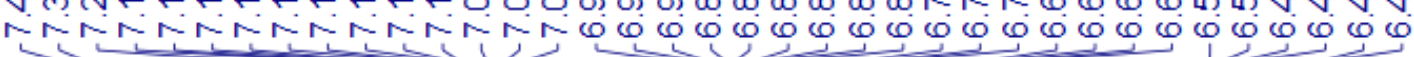

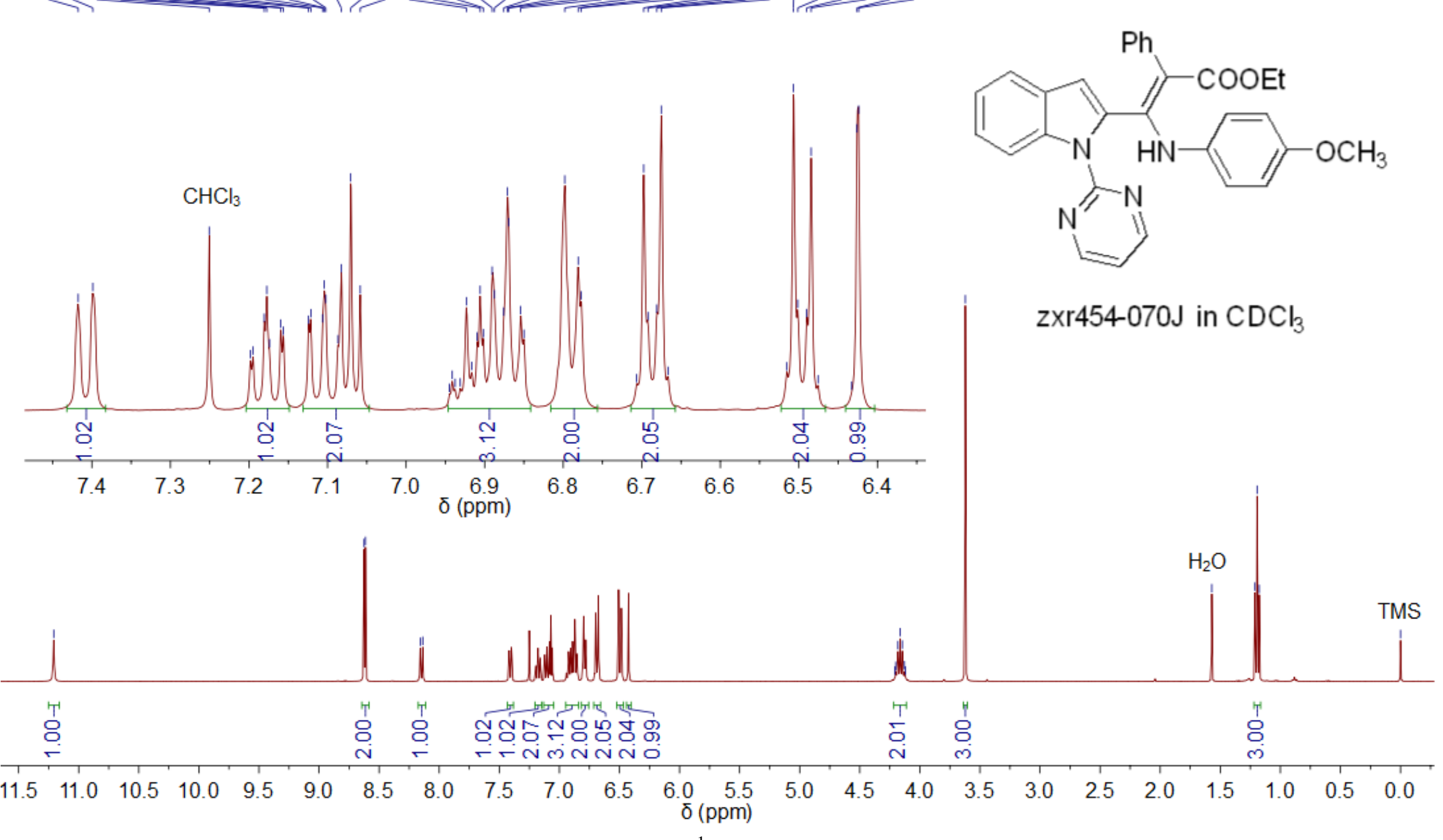

3i $-{ }^{1} \mathrm{H}$ NMR 


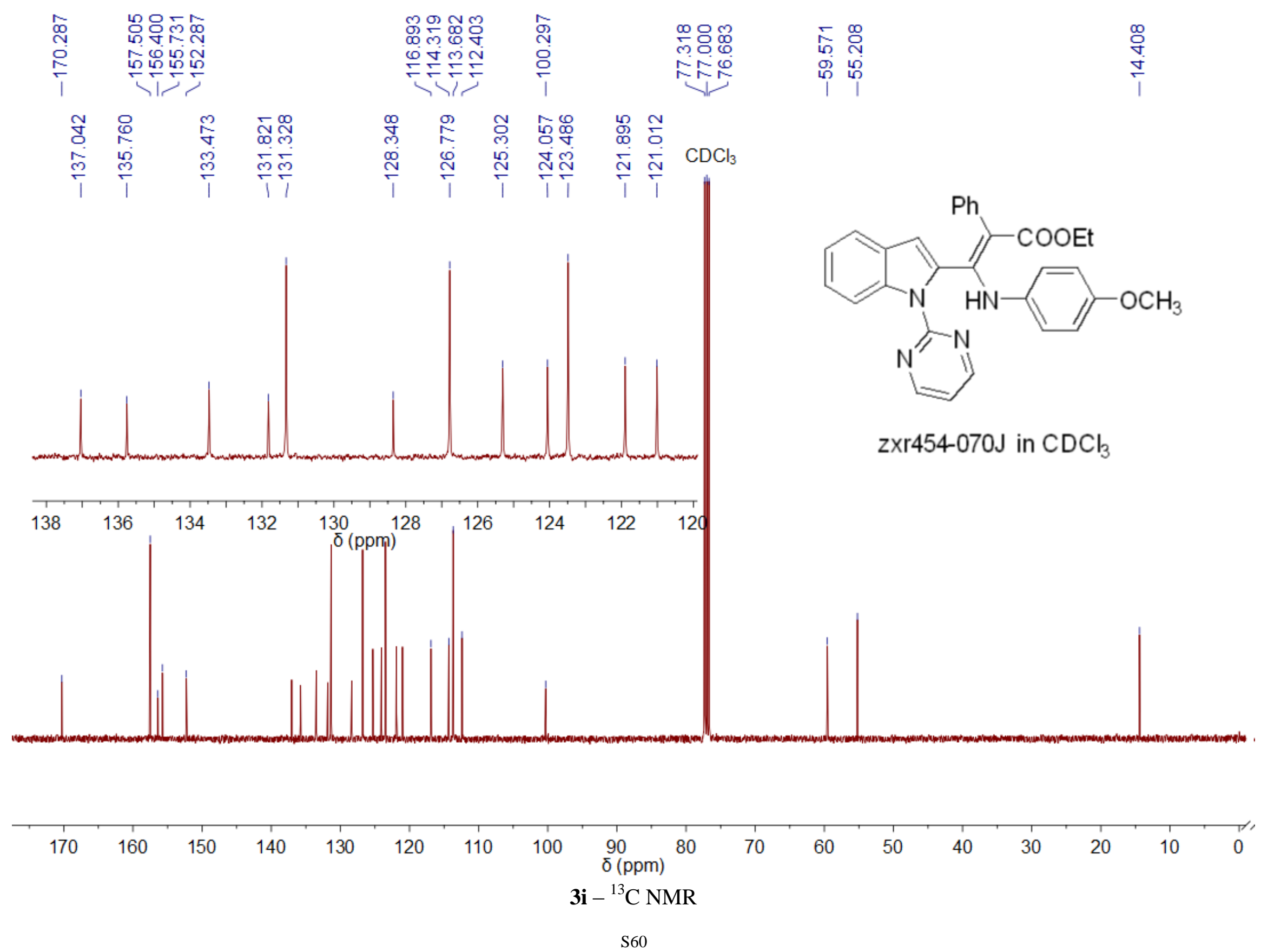




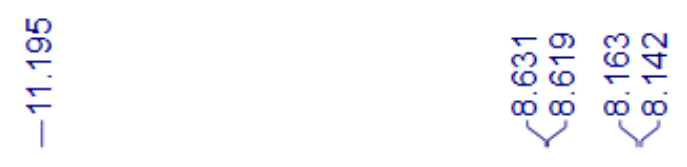

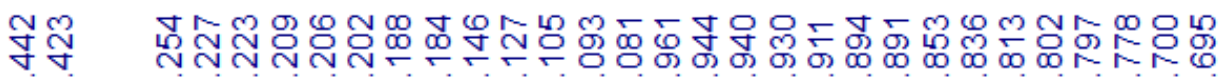

r

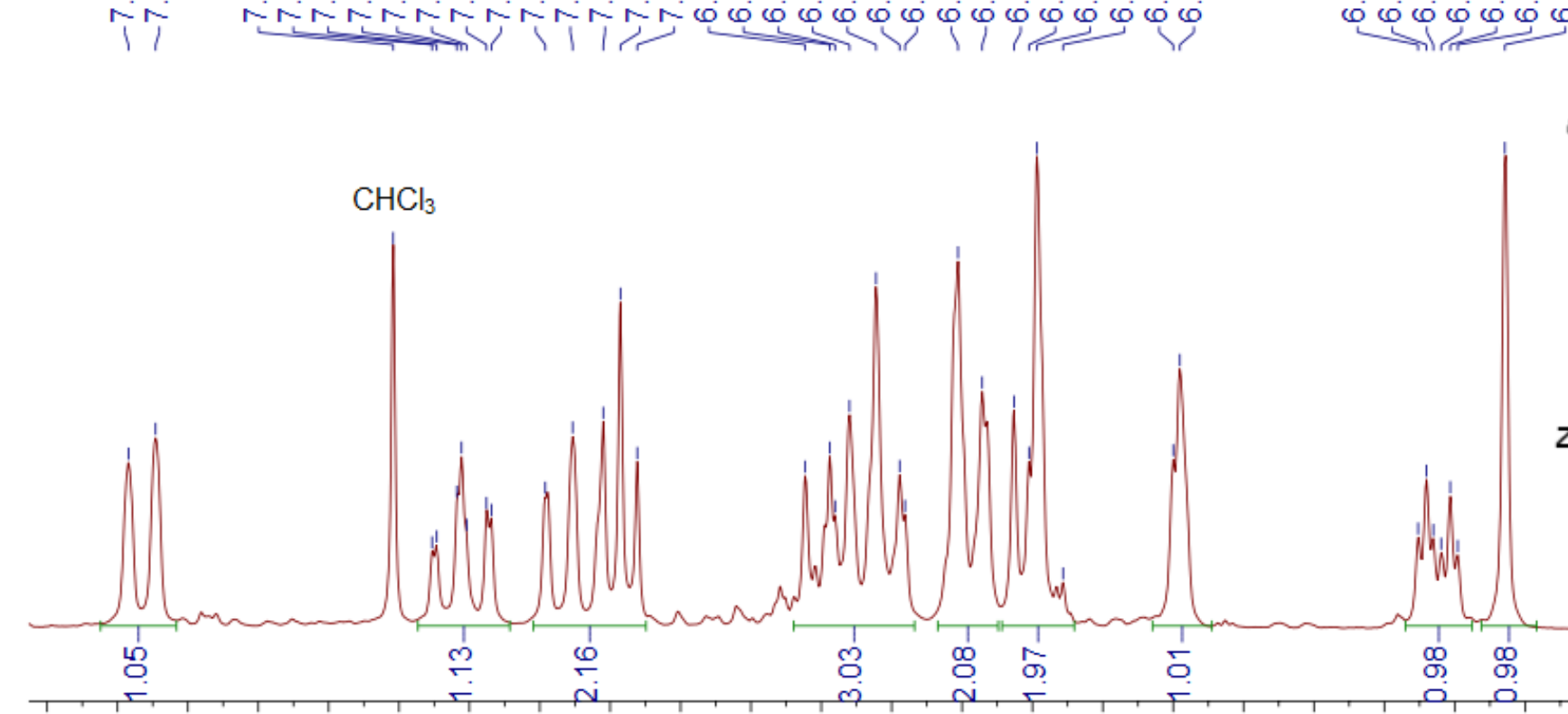

7.507 .457 .407 .357 .307 .257 .207 .157 .107 .057 .006 .956 .906 .856 .806 .756 .706 .656 .606 .556 .506 .45 $\bar{\delta}(\mathrm{ppm})$
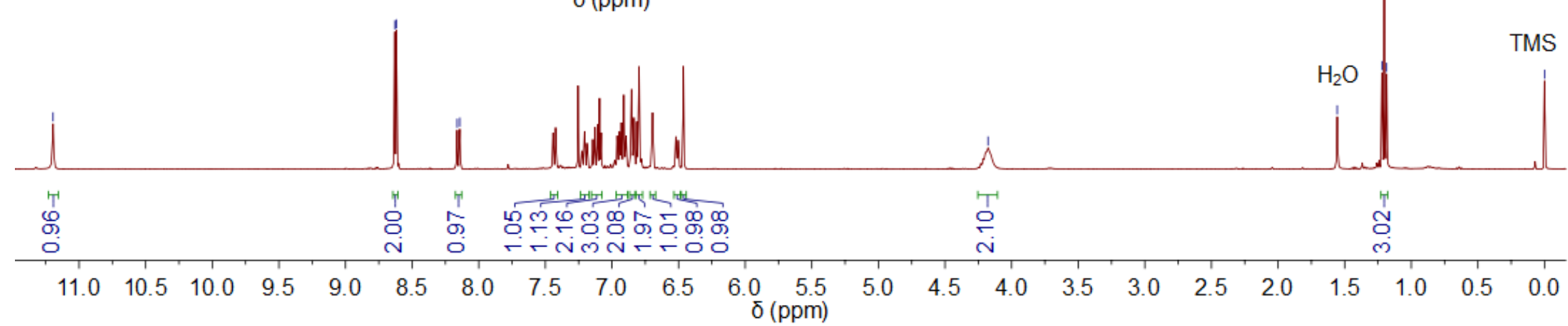

$\mathbf{3 j}-{ }^{1} \mathrm{H}$ NMR

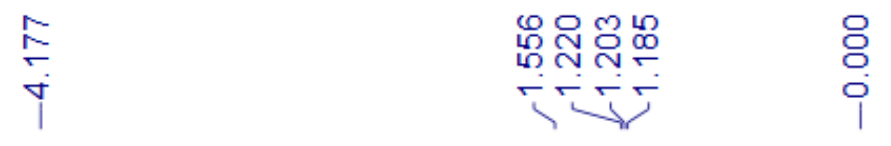

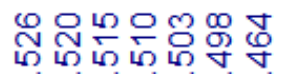<smiles>CCOC(=O)/C(=C/c1cc2ccccc2[nH]1)c1ccccc1</smiles><smiles>Nc1ncccn1</smiles>

zxr454-069E in $\mathrm{CDCb}$
00000 


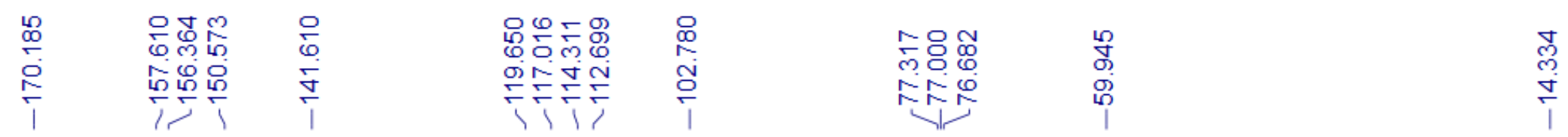

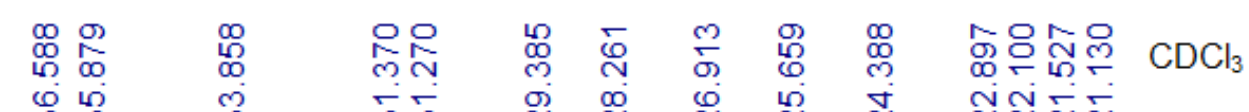

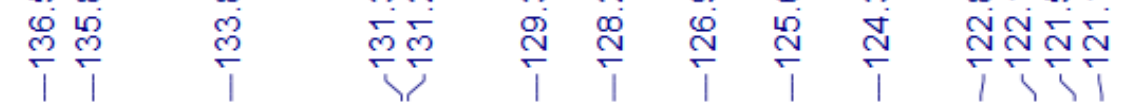

|||||||||||||||||||||||||||| $\mid$<smiles>CCOC(=O)C(=C(Nc1cccc(Cl)c1)c1cc2ccccc2n1-c1ncccn1)c1ccccc1</smiles>

zxr454-069E in $\mathrm{CDCb}_{3}$

$137136135134133132131 \begin{gathered}130129128127126125124123122121 \\ \bar{\delta}(\mathrm{ppm})\end{gathered}$

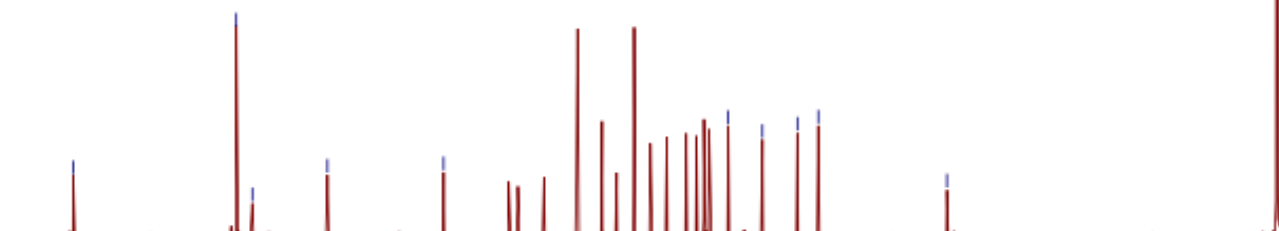

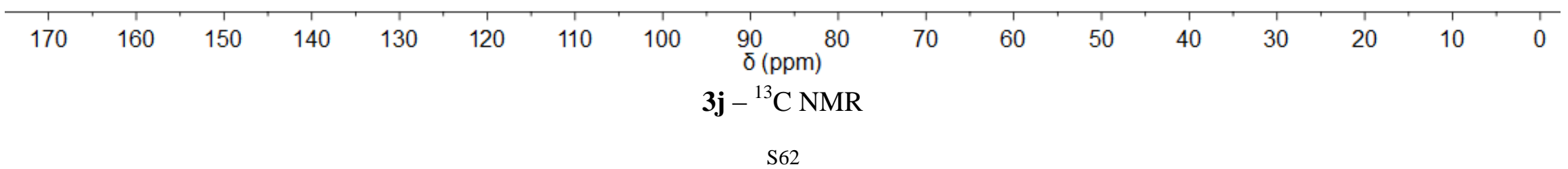




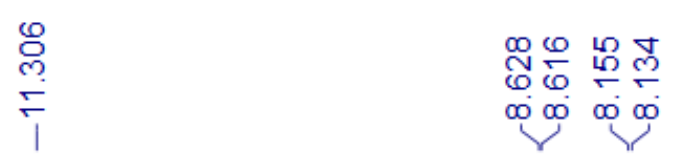

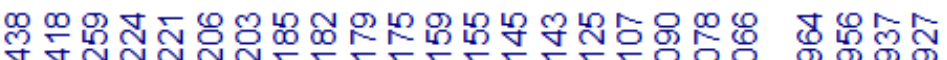

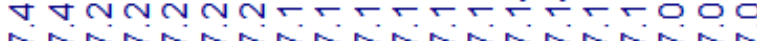

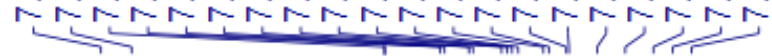

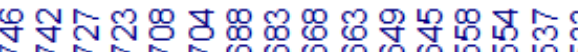

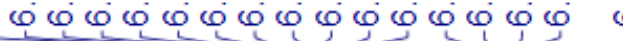

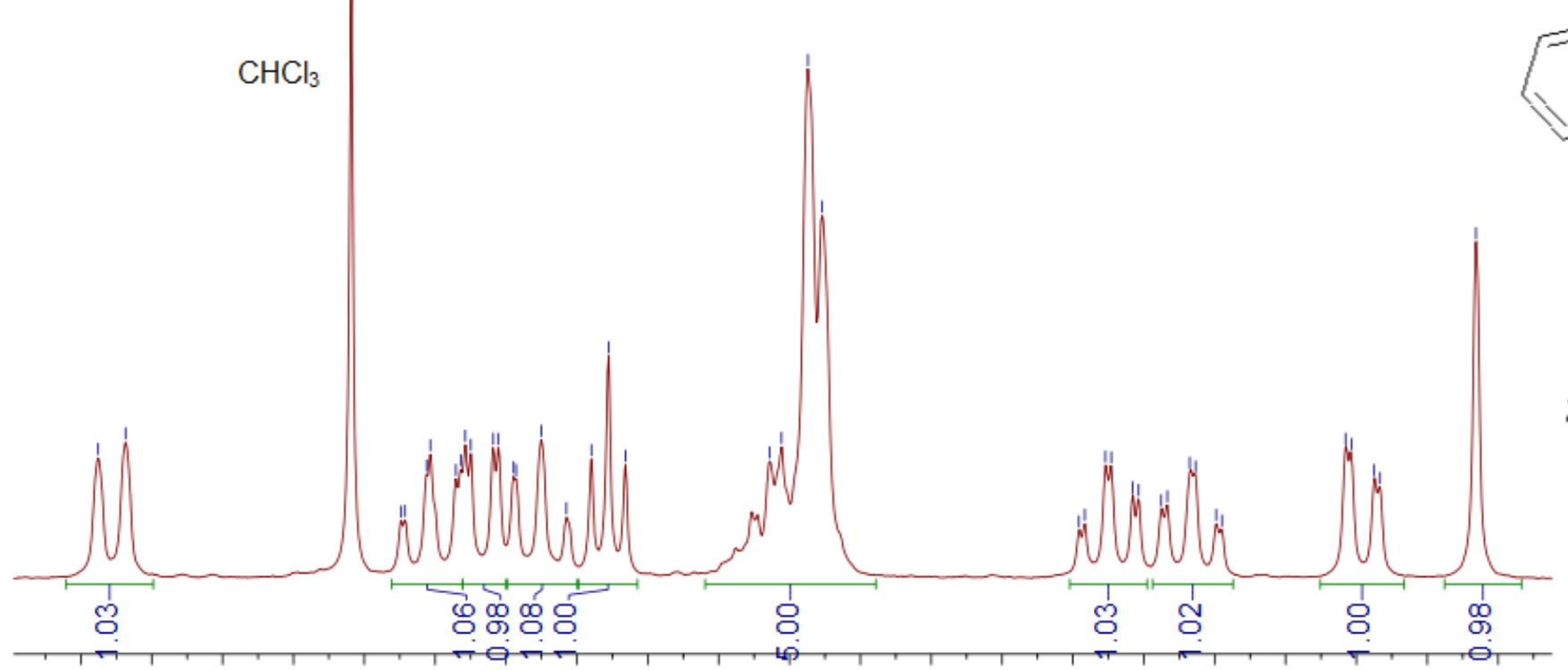

7.457 .407 .357 .307 .257 .207 .157 .107 .057 .006 .956 .906 .856 .806 .756 .706 .656 .606 .556 .506 .45 $\delta(\mathrm{ppm})$

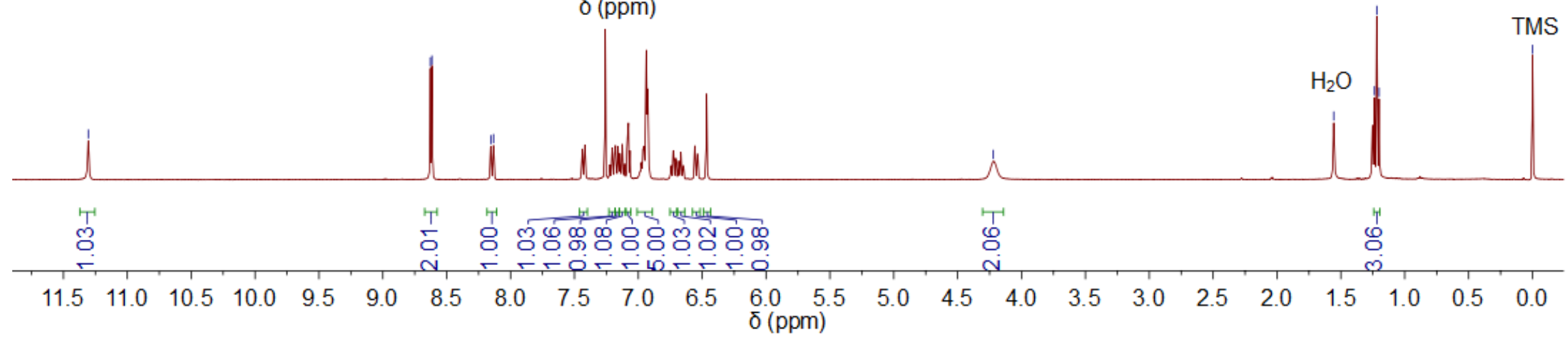

3k $-{ }^{1} \mathrm{H}$ NMR 


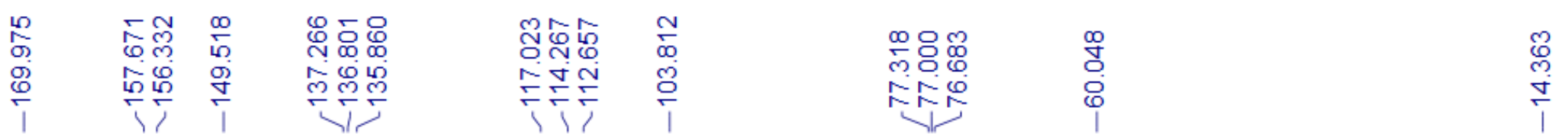

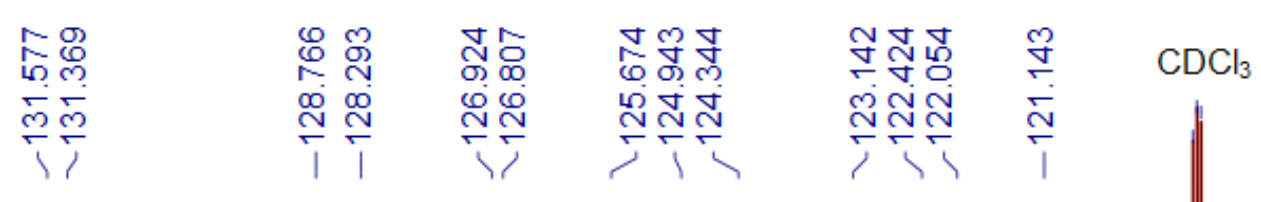

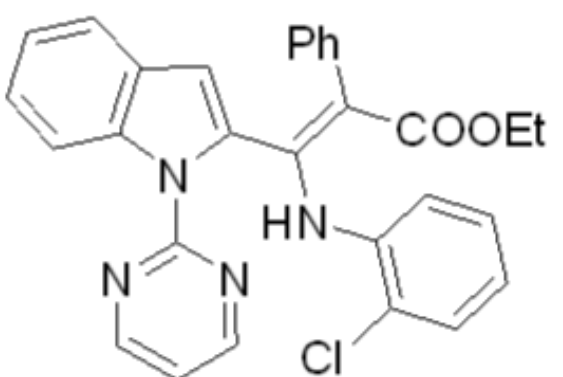

zxr454-069F in $\mathrm{CDCl}_{3}$

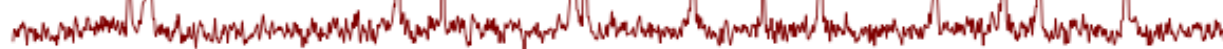

$\begin{array}{llllllllllll}132 & 131 & 130 & 129 & 128 & \begin{array}{c}127 \\ \delta(\mathrm{ppm})\end{array} & 125 & 124 & 123 & 122 & 121\end{array}$

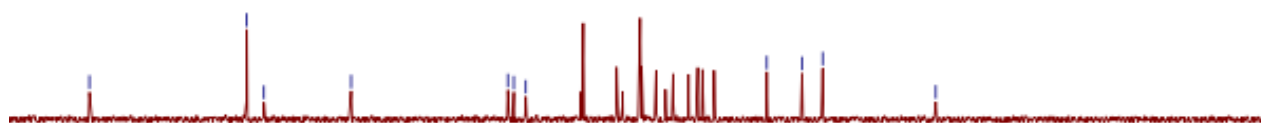

\begin{tabular}{|c|c|c|c|c|c|c|c|c|c|c|c|c|c|c|c|c|}
\hline 170 & 160 & 150 & 140 & 130 & 120 & 110 & 100 & ${ }^{90}(\mathrm{ppm}){ }^{80}$ & 70 & 60 & 50 & 40 & 30 & 20 & 10 & 0 \\
\hline
\end{tabular}



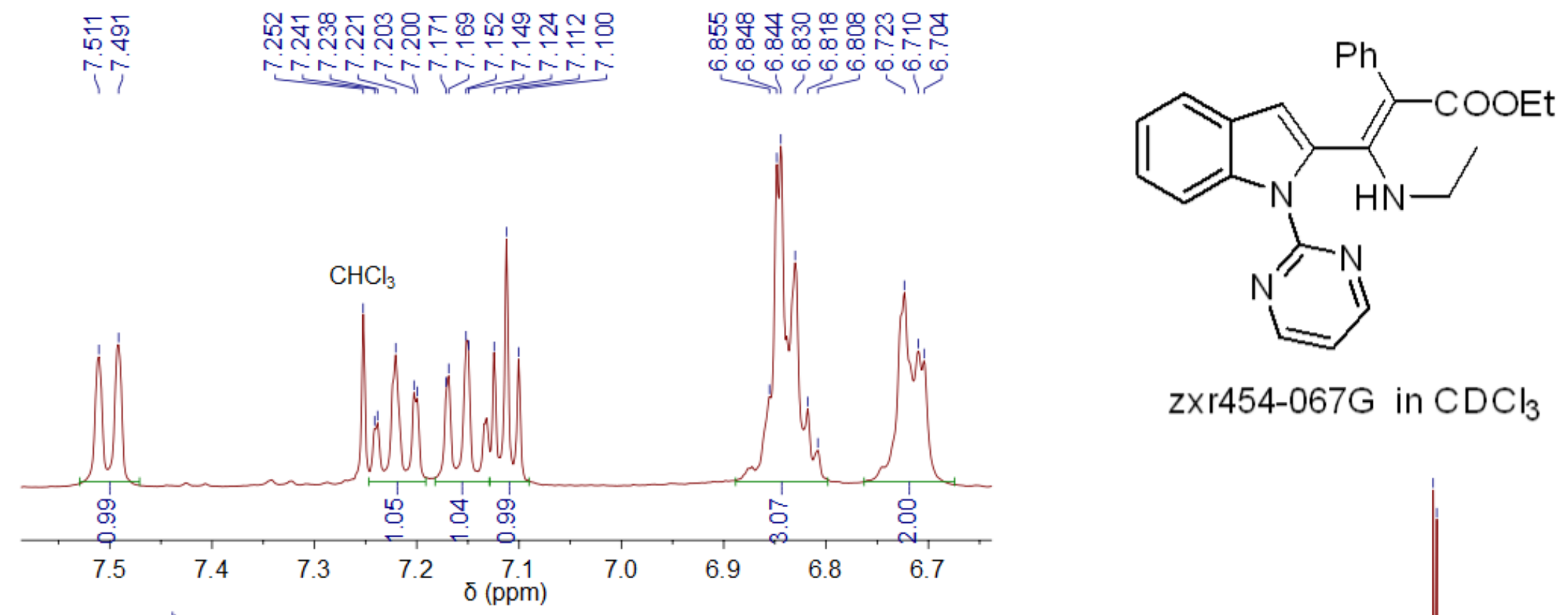

zxr454-067G in $\mathrm{CDCl}_{3}$
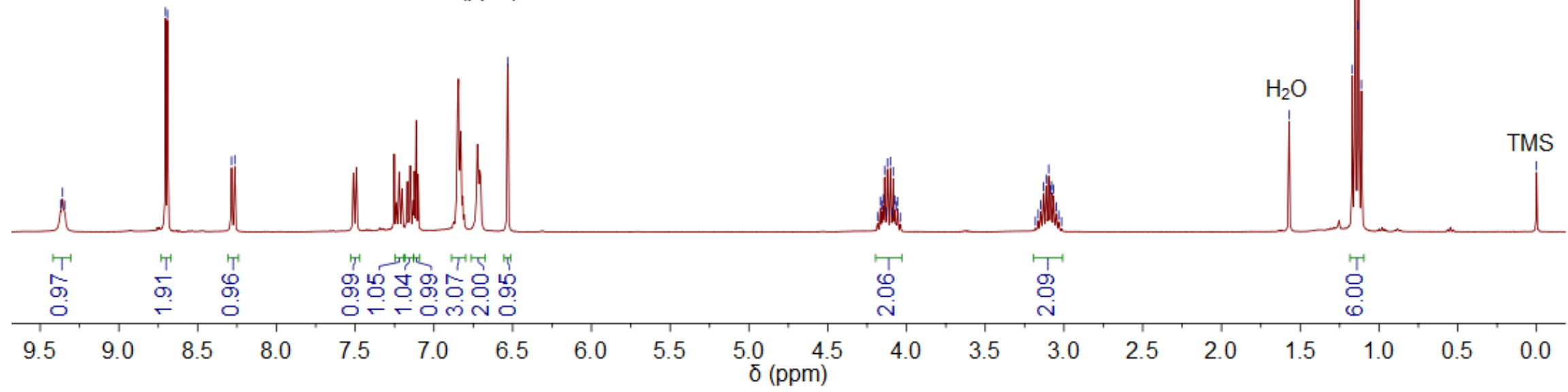

3I- ${ }^{1} \mathrm{H}$ NMR 


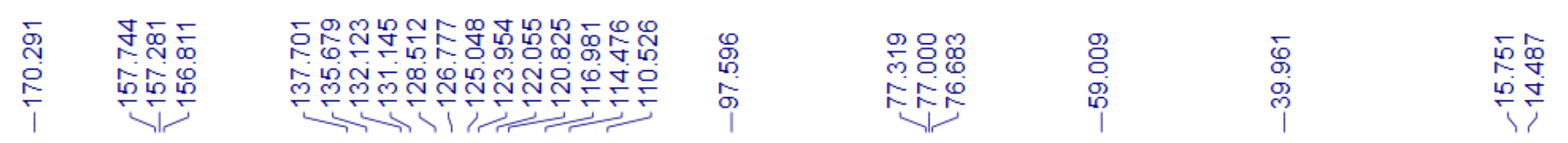

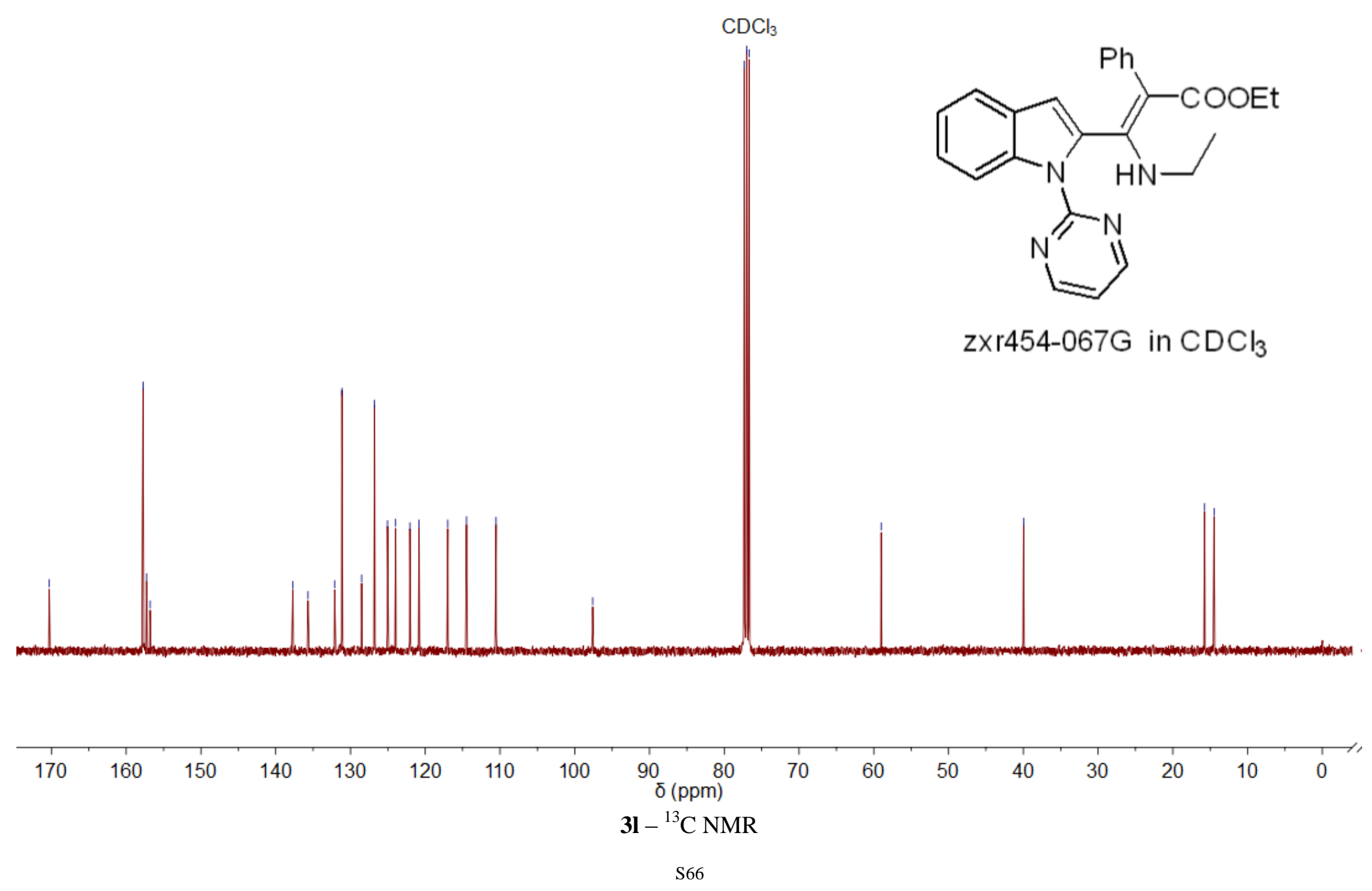




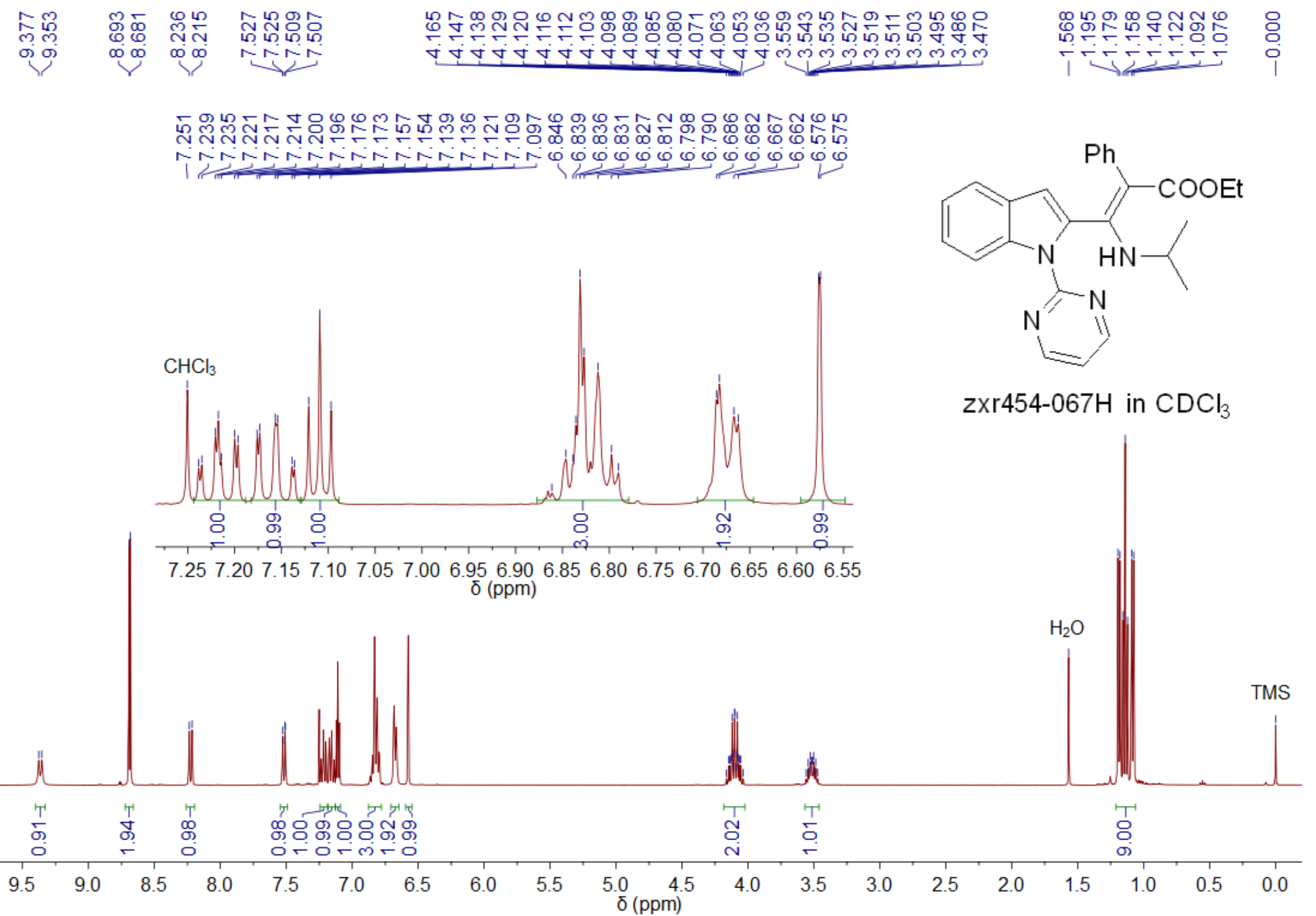

3m $-{ }^{1} \mathrm{H}$ NMR 
$\mathrm{CDCl}_{3}$

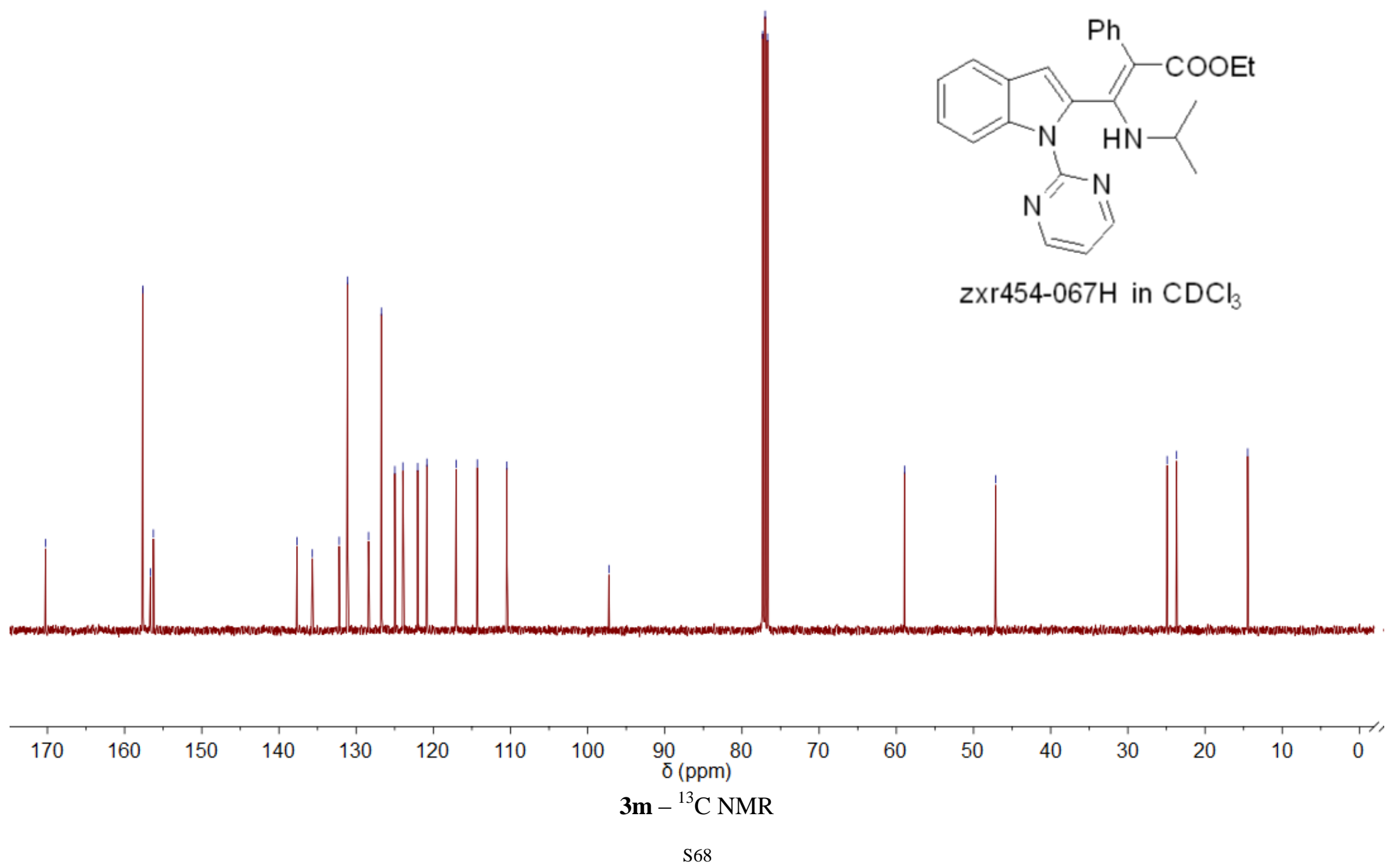




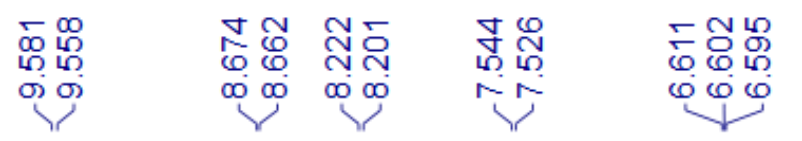

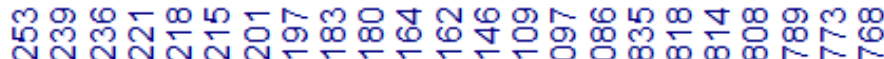

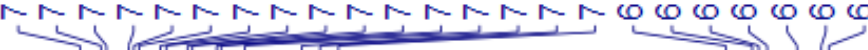
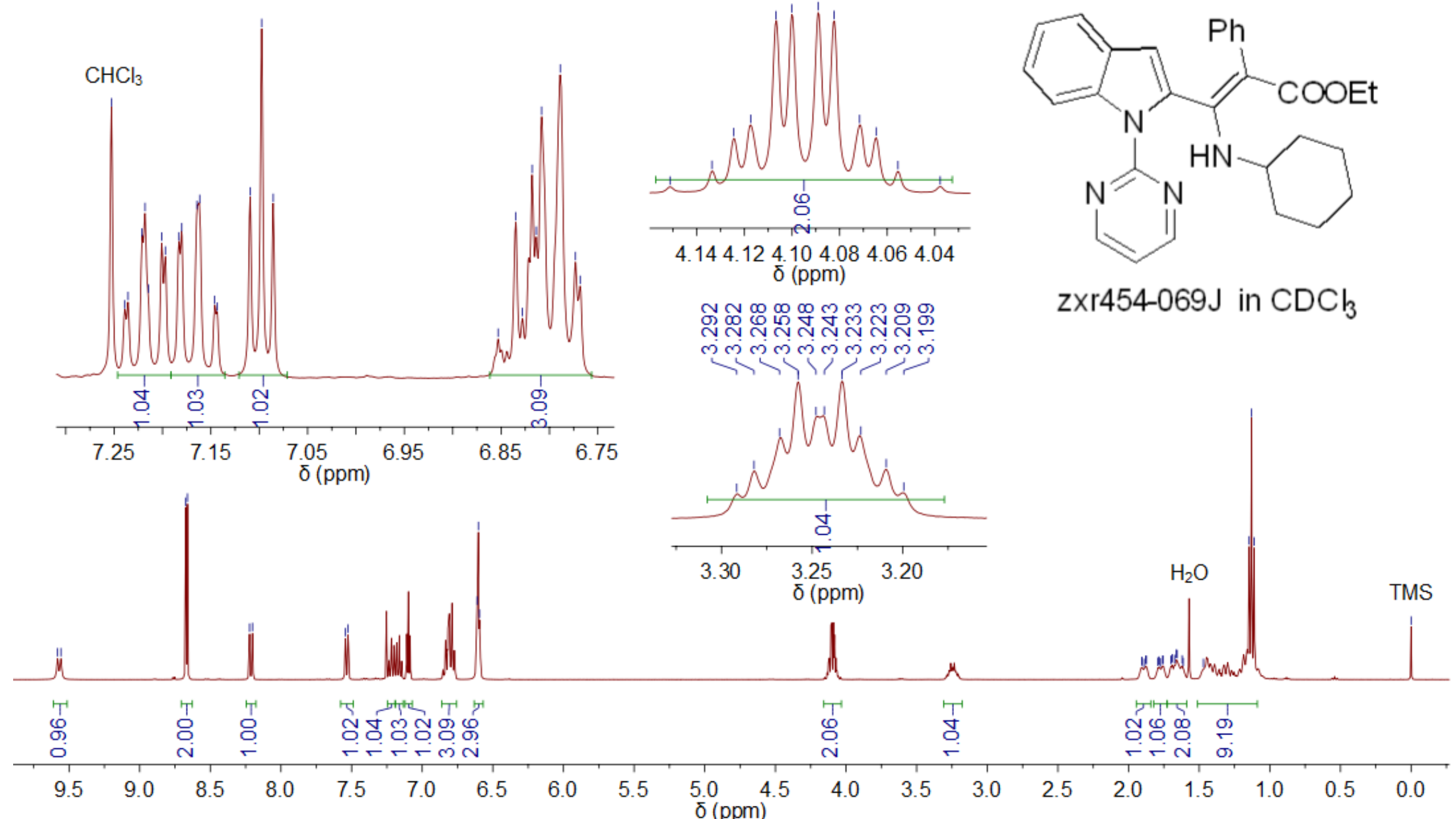

3n $-{ }^{1} \mathrm{H}$ NMR

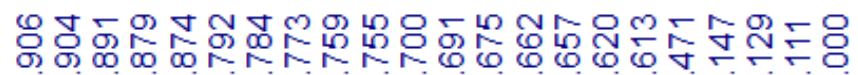

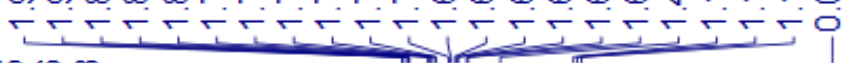

点 虫

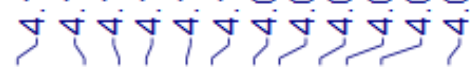

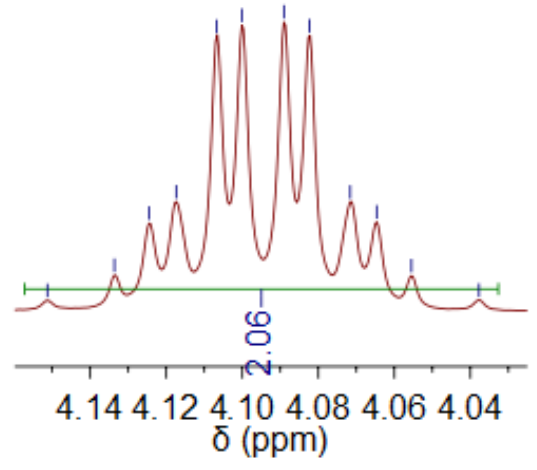

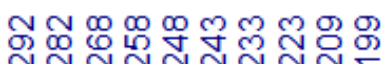

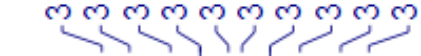

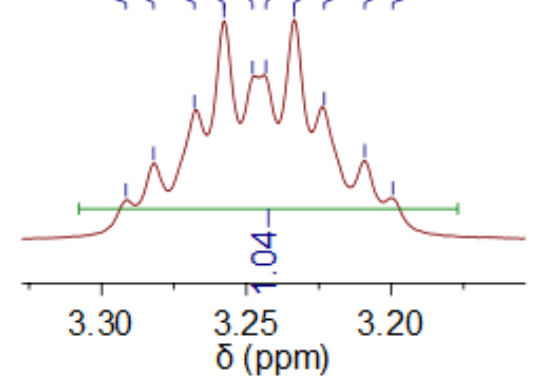

zxr454-069J in $\mathrm{CDCl}_{3}$
(1)

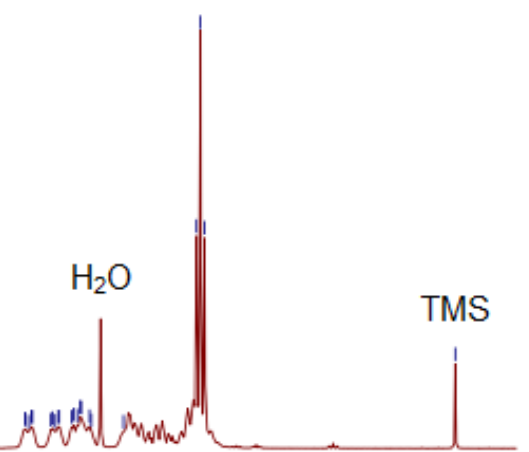




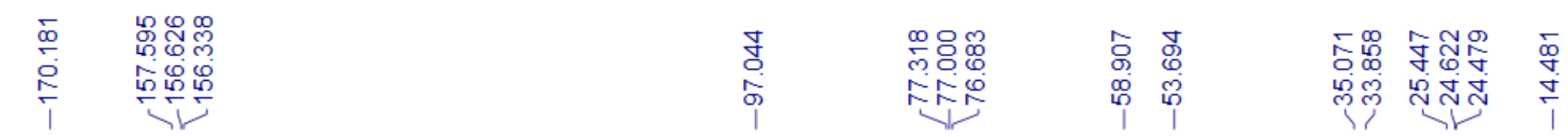

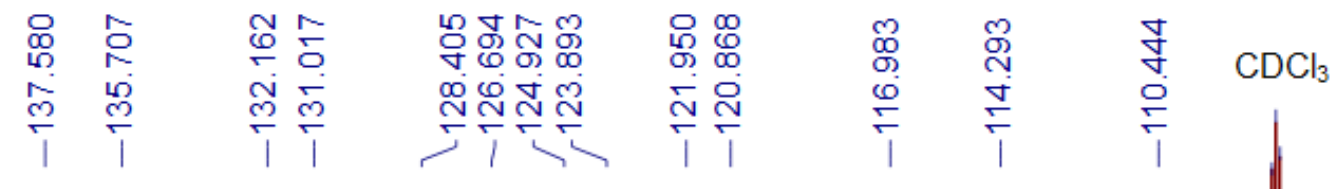

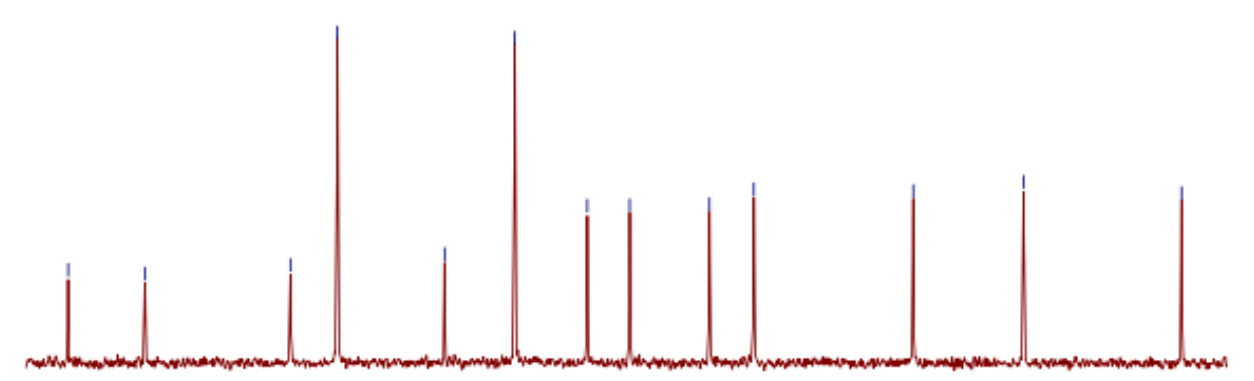

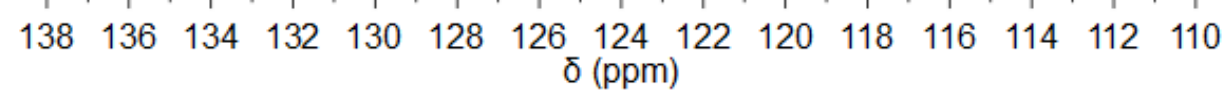
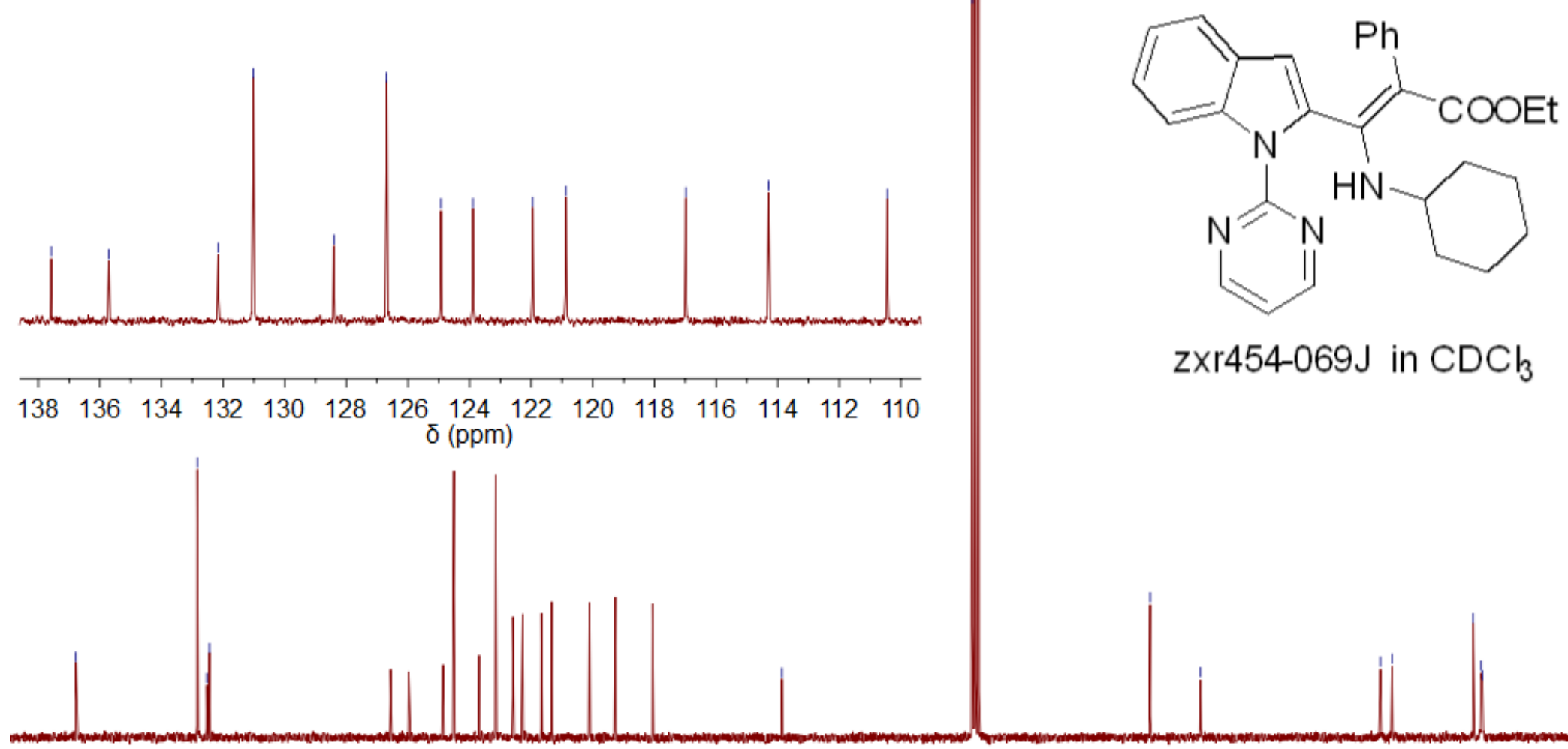

zxr454-069J in $\mathrm{CDCl}_{3}$

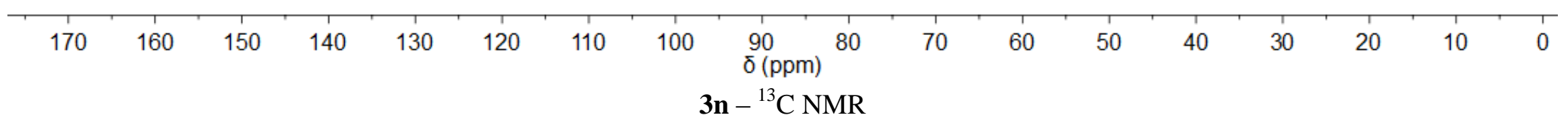




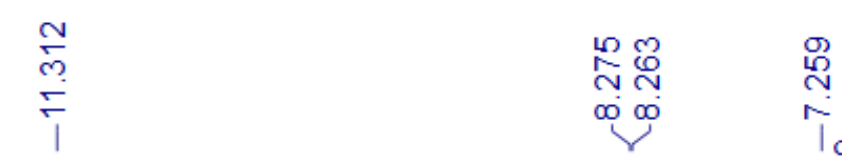

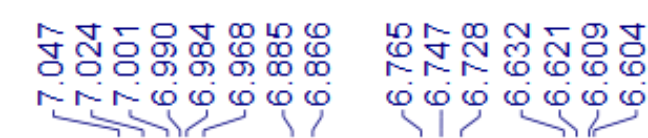

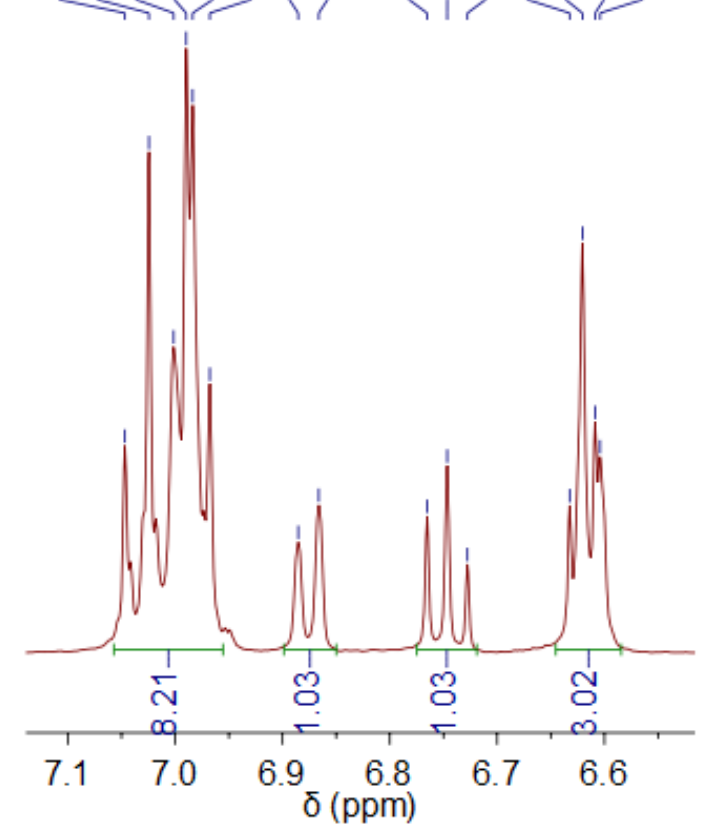

罩

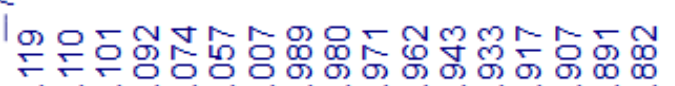

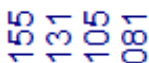

लंखेल

ஸृ

웅

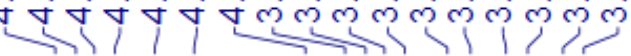

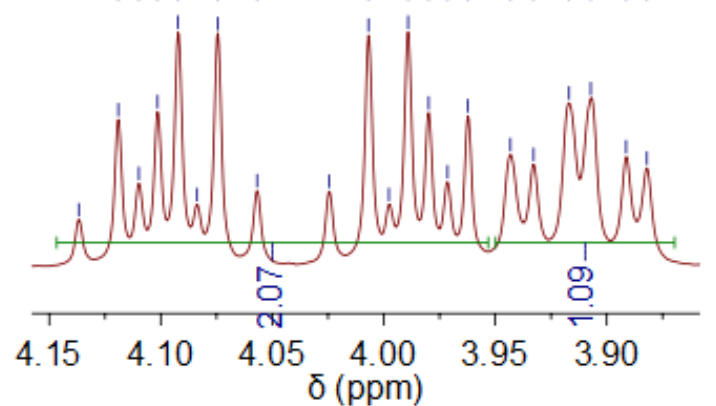

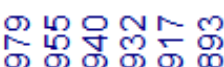

का के के क

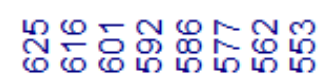

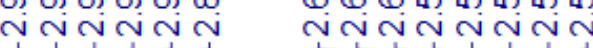
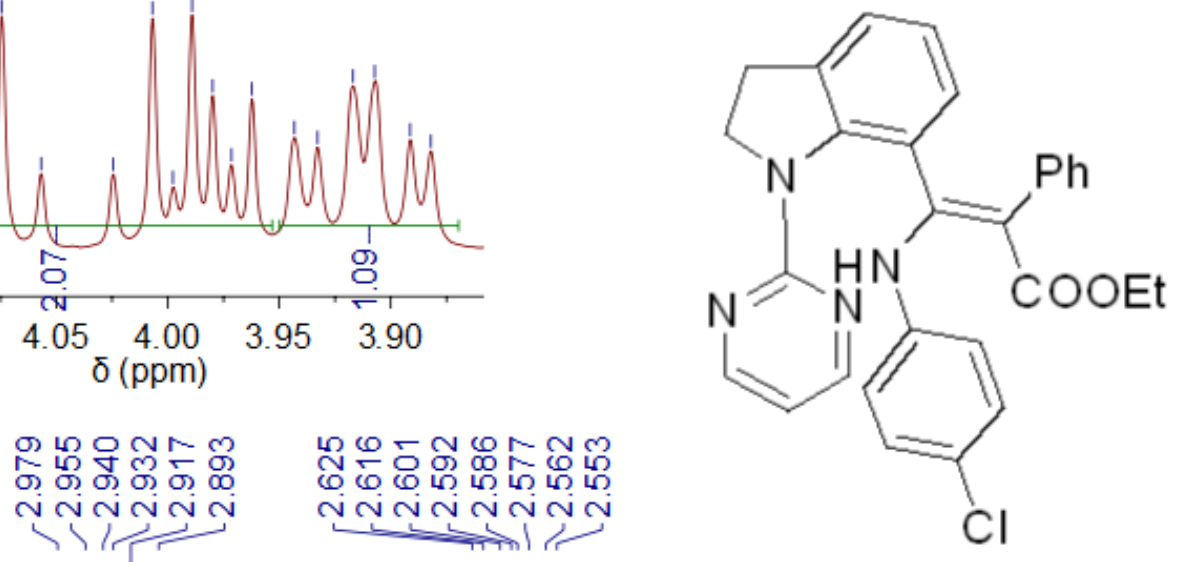

zxr454-082A in $\mathrm{CDCl}_{3}$
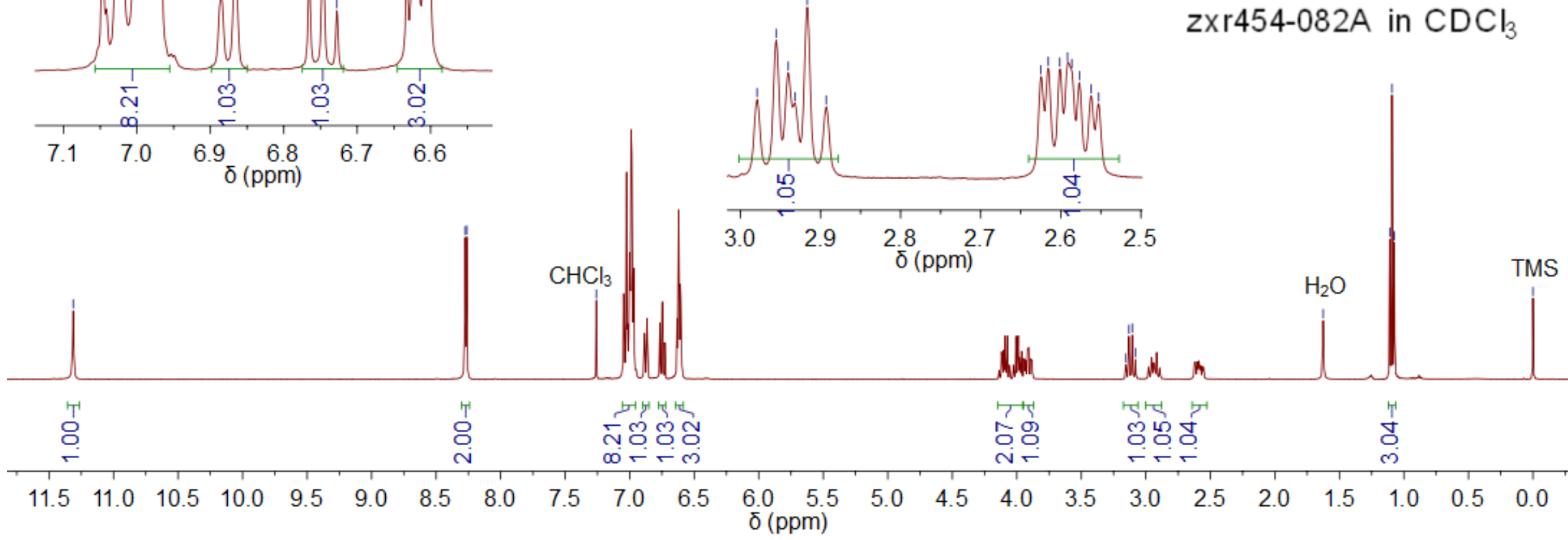

3o $-{ }^{1} \mathrm{H}$ NMR 

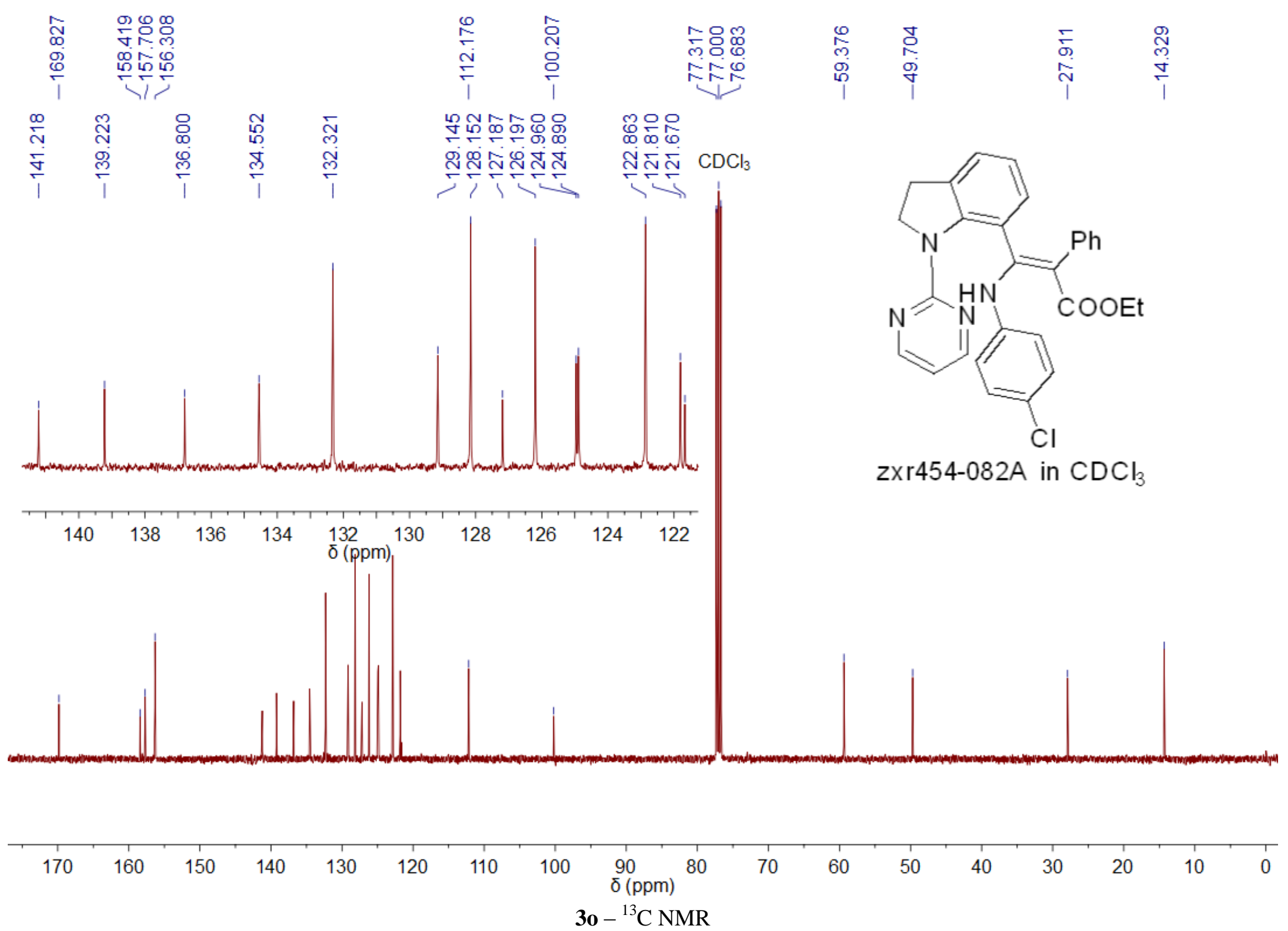


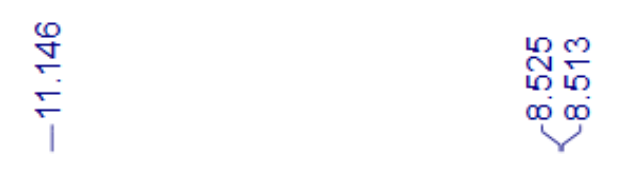

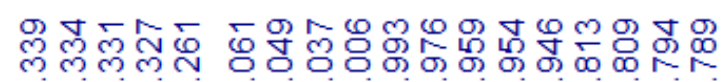

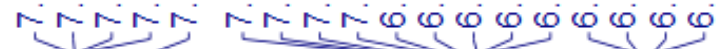
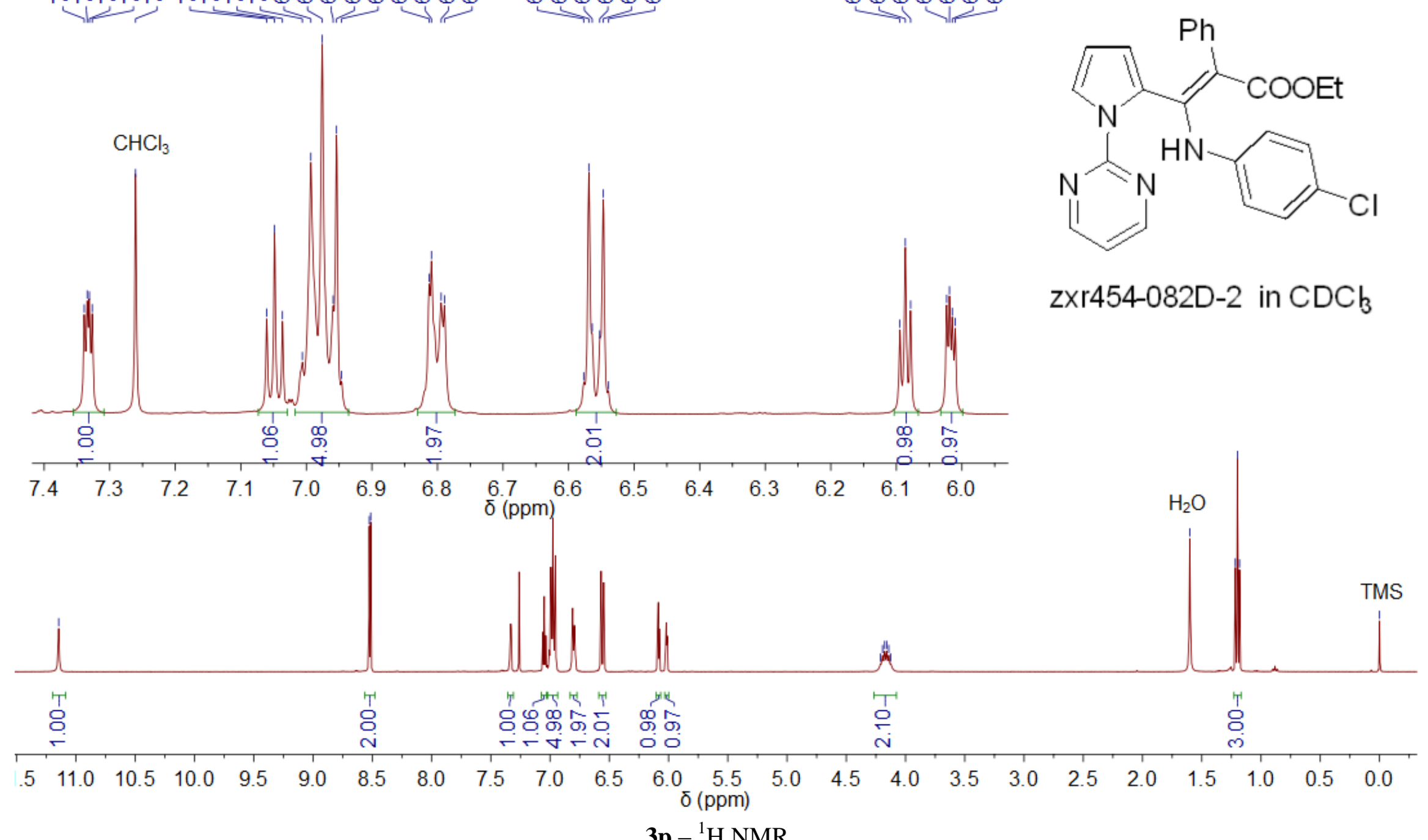

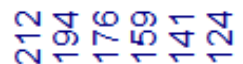

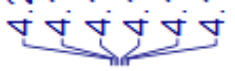

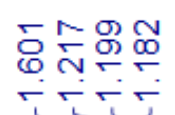

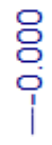

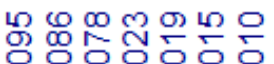

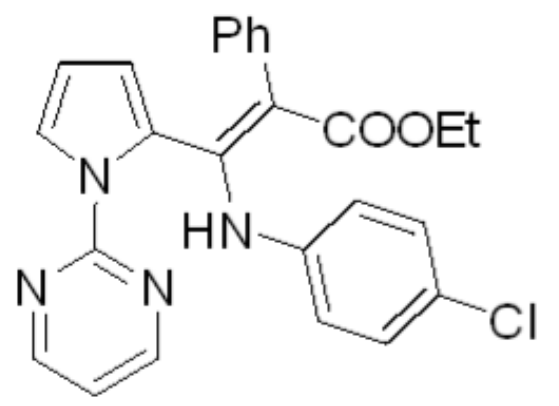

zxr454-082D-2 in $\mathrm{CDCb}_{3}$ 


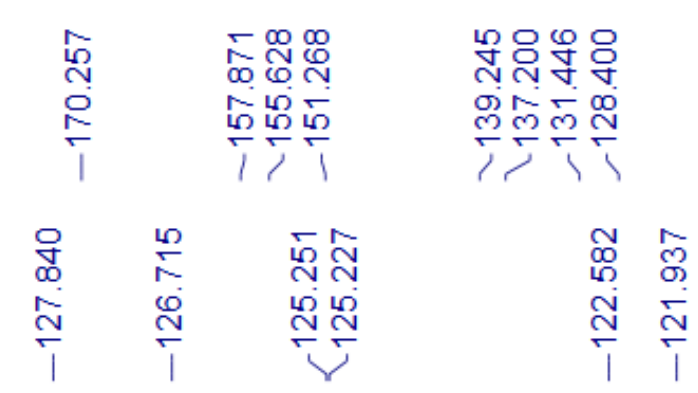

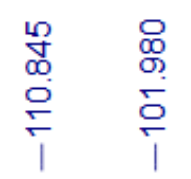

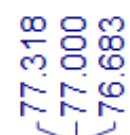

早

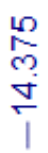
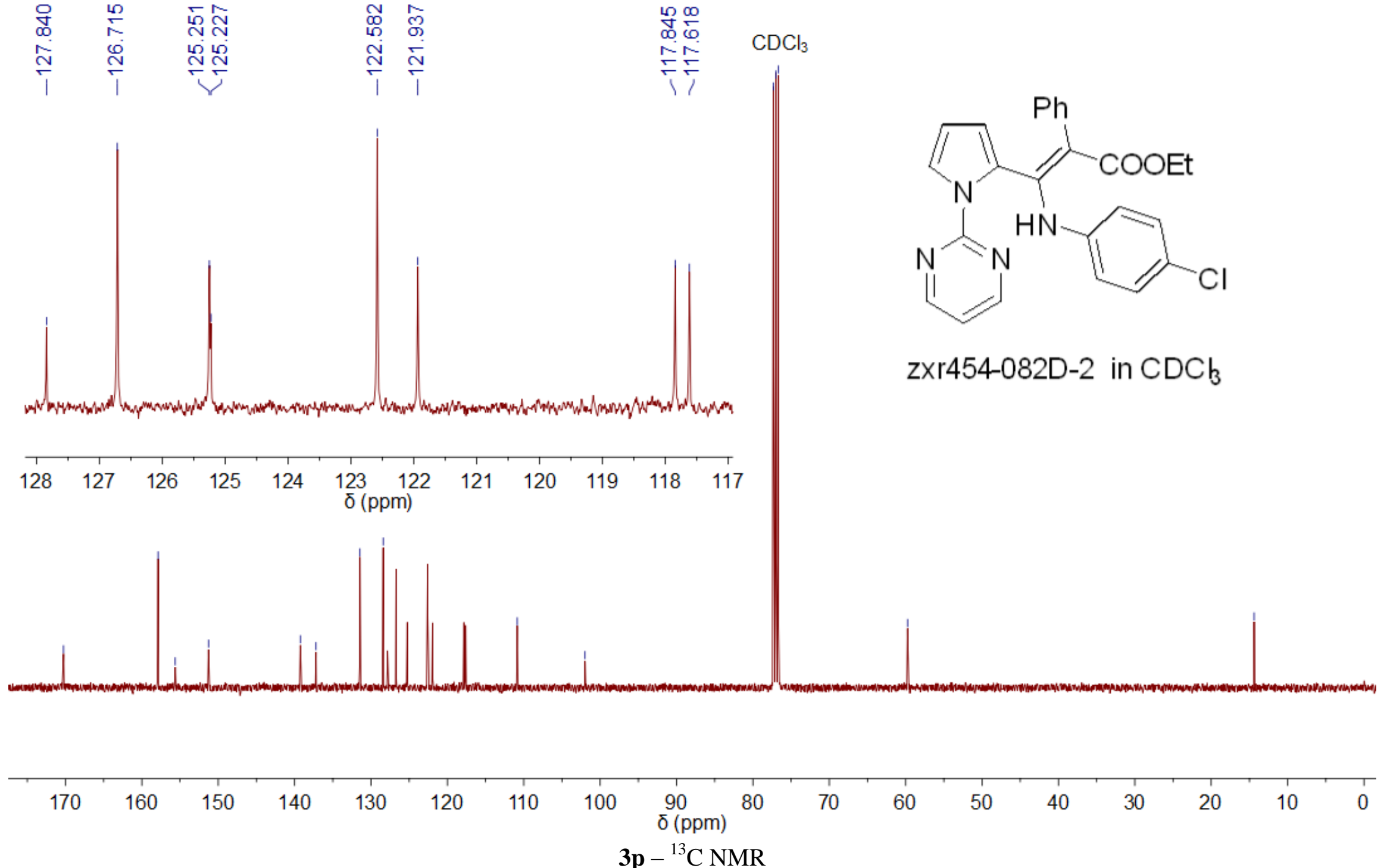


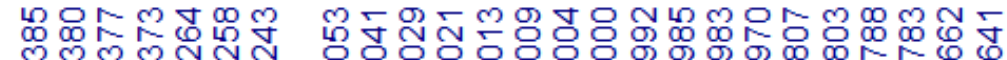

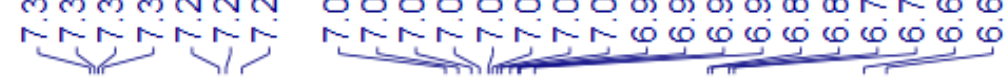

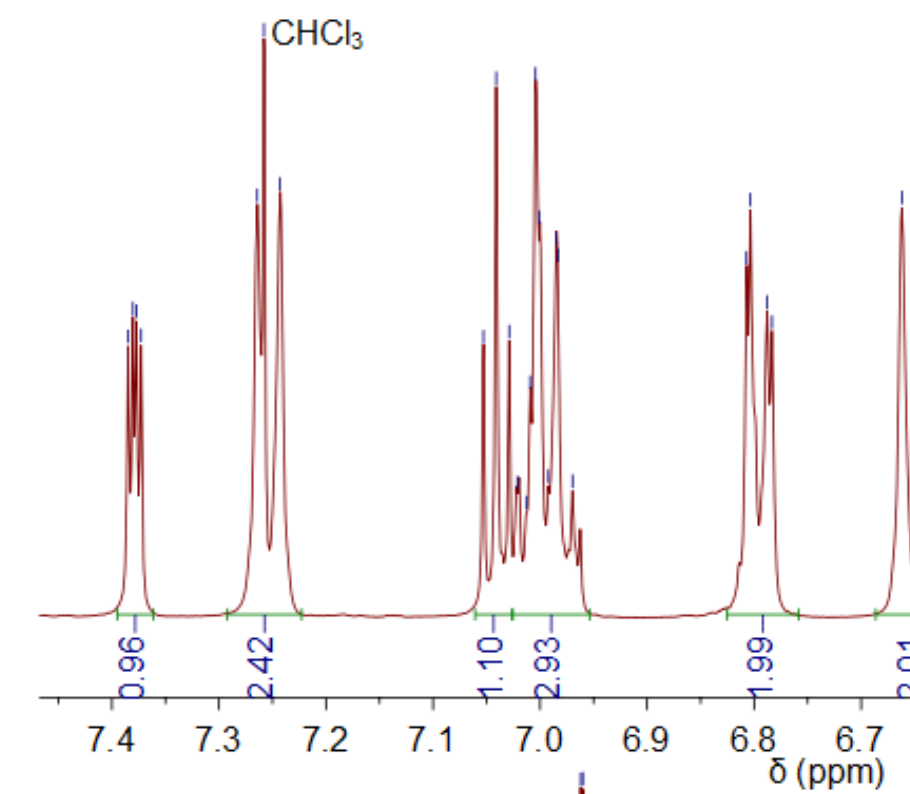

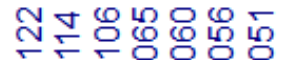

ம

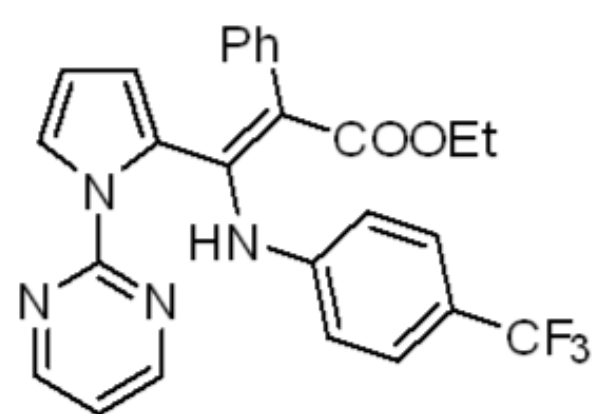

zxr454-113A in $\mathrm{CDCl}_{3}$

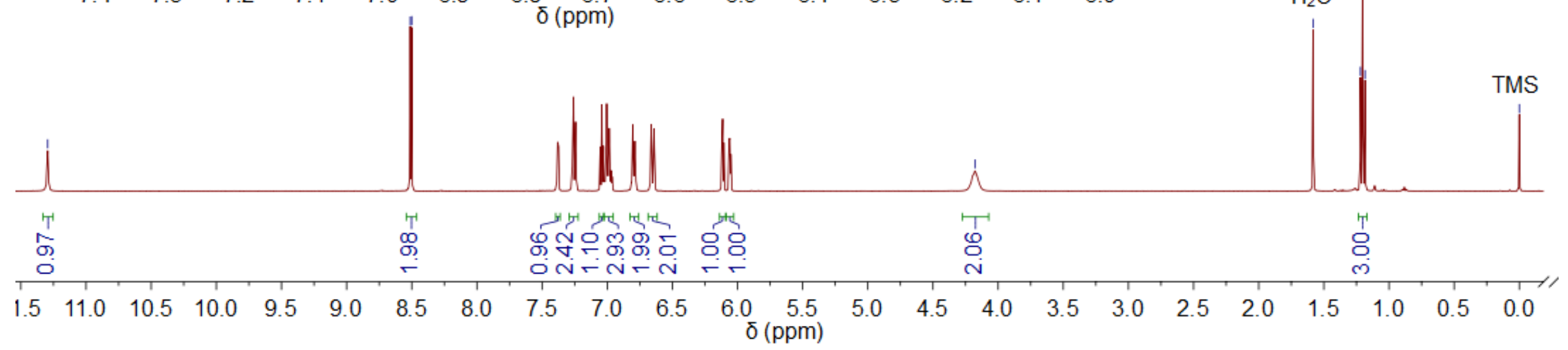

$3 \mathbf{q}-{ }^{1} \mathrm{H}$ NMR 


\begin{tabular}{|c|c|c|c|c|c|c|}
\hline 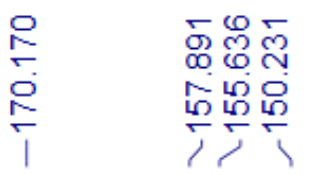 & $\begin{array}{l}\frac{m}{\infty} \\
\stackrel{m}{+} \\
\stackrel{j}{+}\end{array}$ & 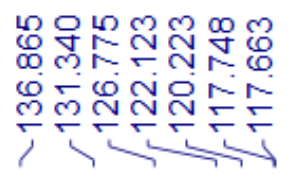 & 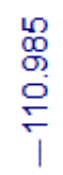 & $\begin{array}{l}\infty \\
0 \\
0 \\
\stackrel{0}{\circ} \\
\end{array}$ & 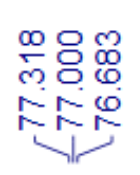 & $\begin{array}{l}0 \\
\infty \\
\sigma \\
0 \\
0 \\
1\end{array}$ \\
\hline
\end{tabular}
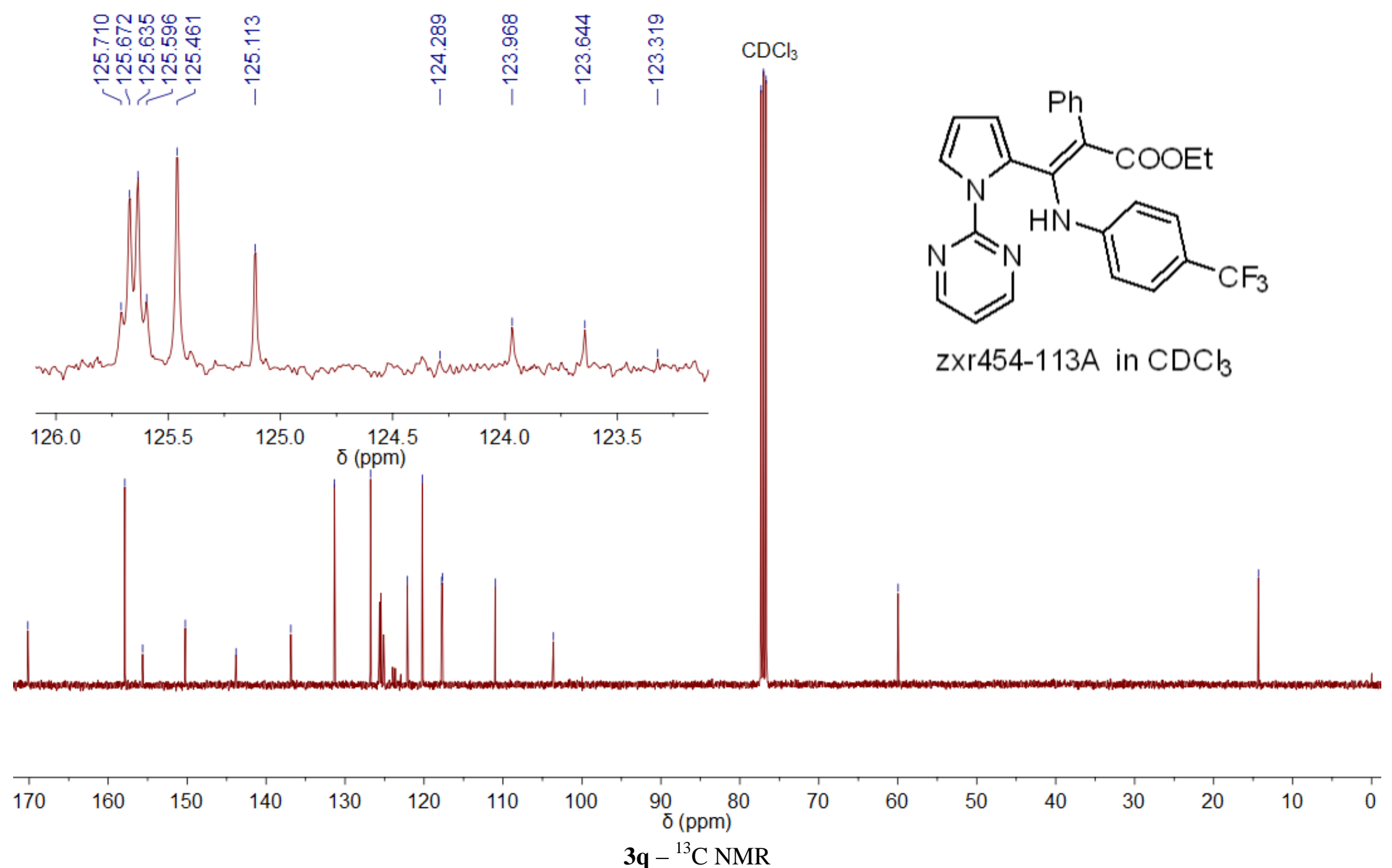


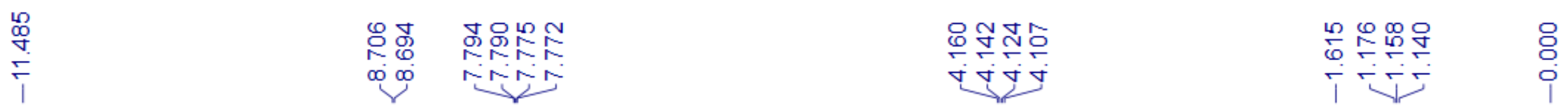

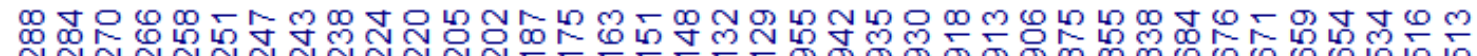

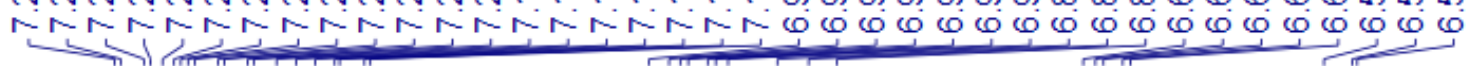
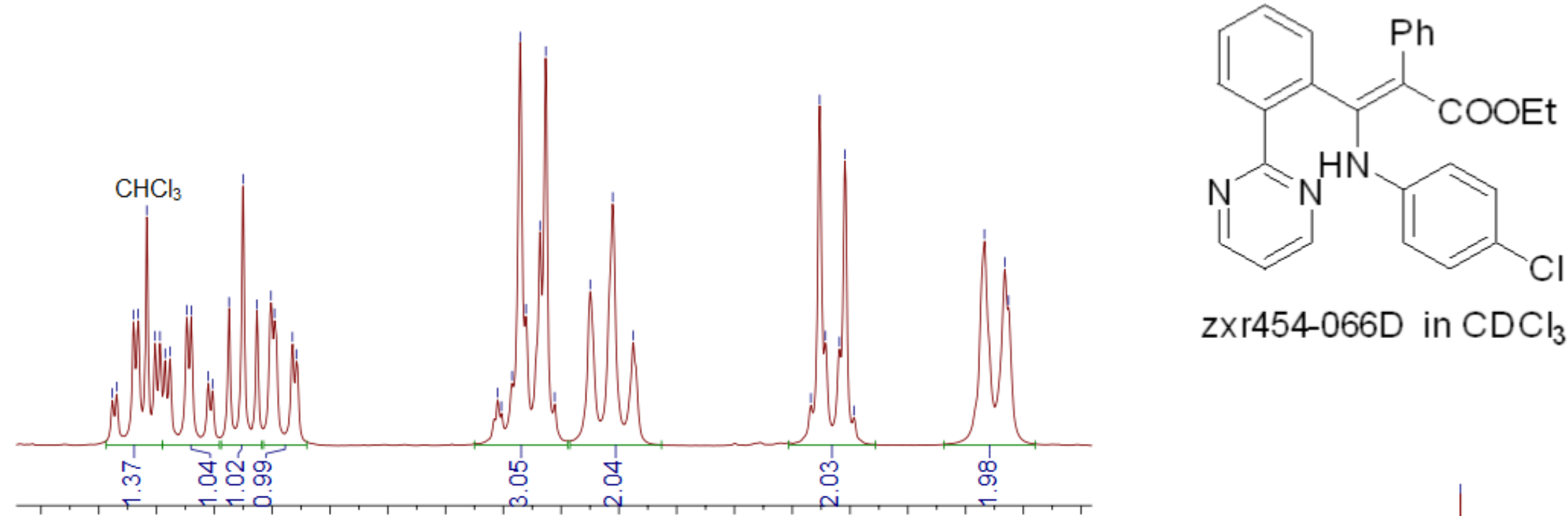

zxr454-066D in $\mathrm{CDCl}_{3}$

7.357 .307 .257 .207 .157 .107 .057 .006 .956 .906 .856 .806 .756 .706 .656 .606 .556 .506 .45 $\delta(\mathrm{ppm})$

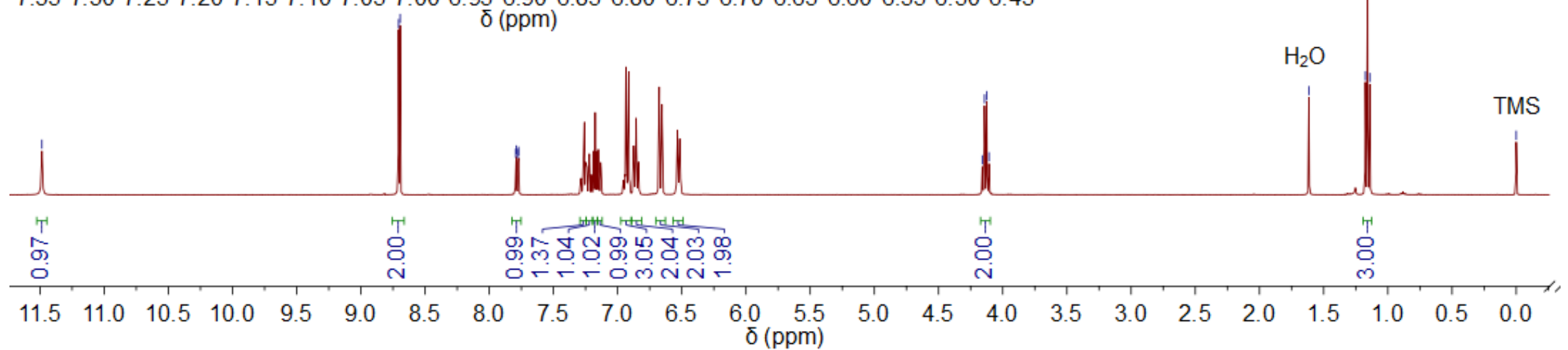

$\mathbf{5 a}-{ }^{1} \mathrm{H}$ NMR 

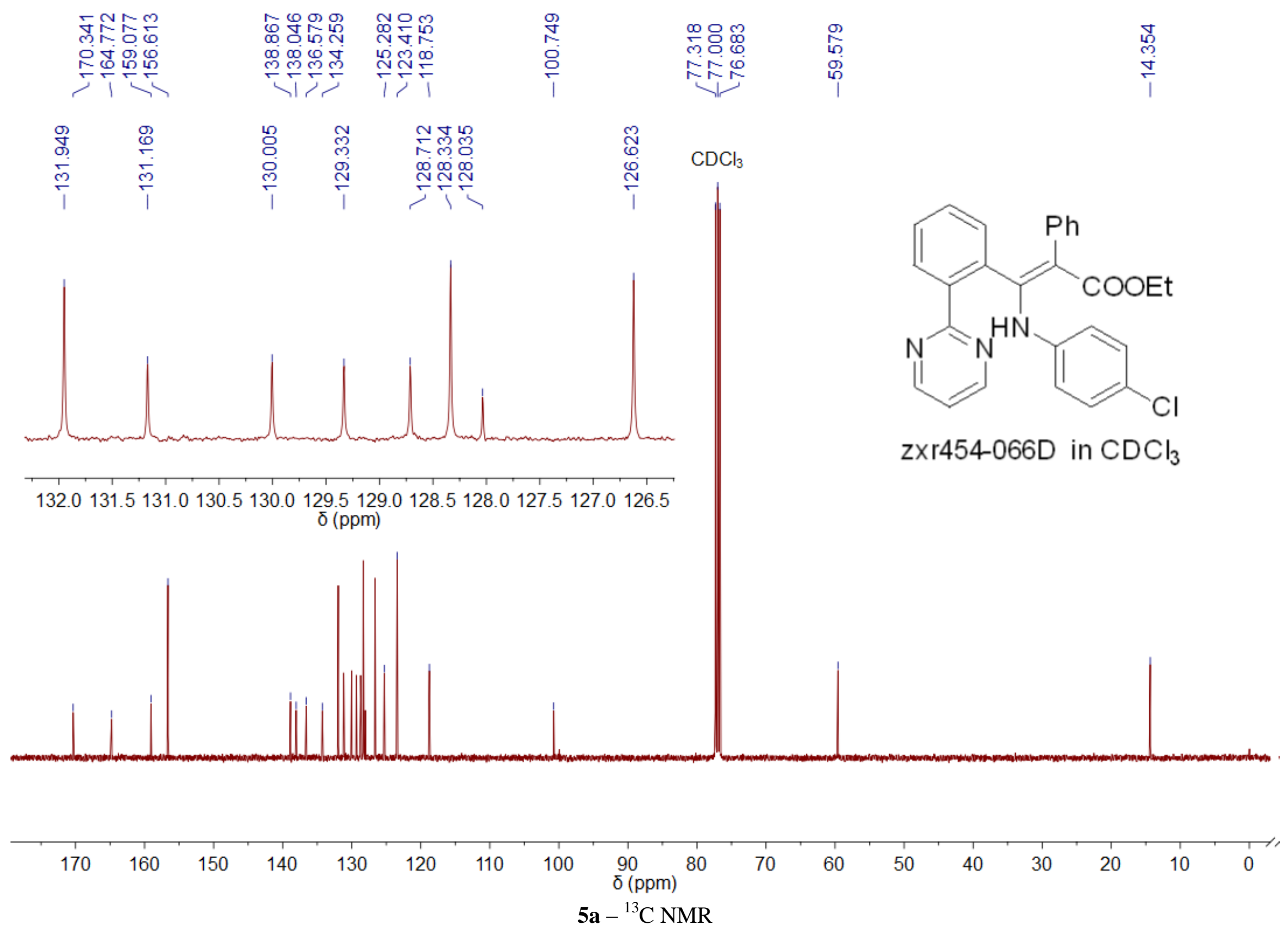


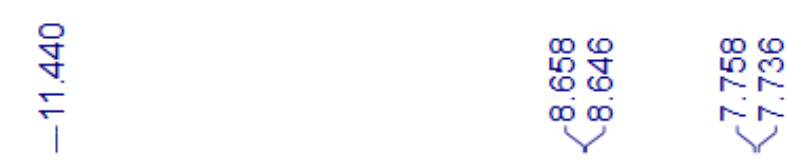

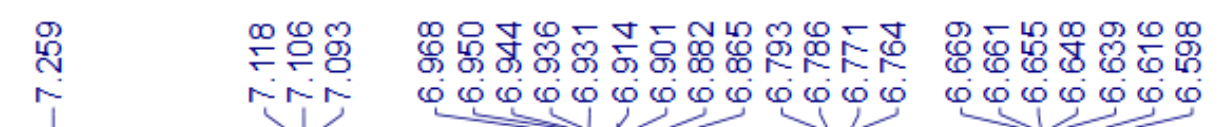

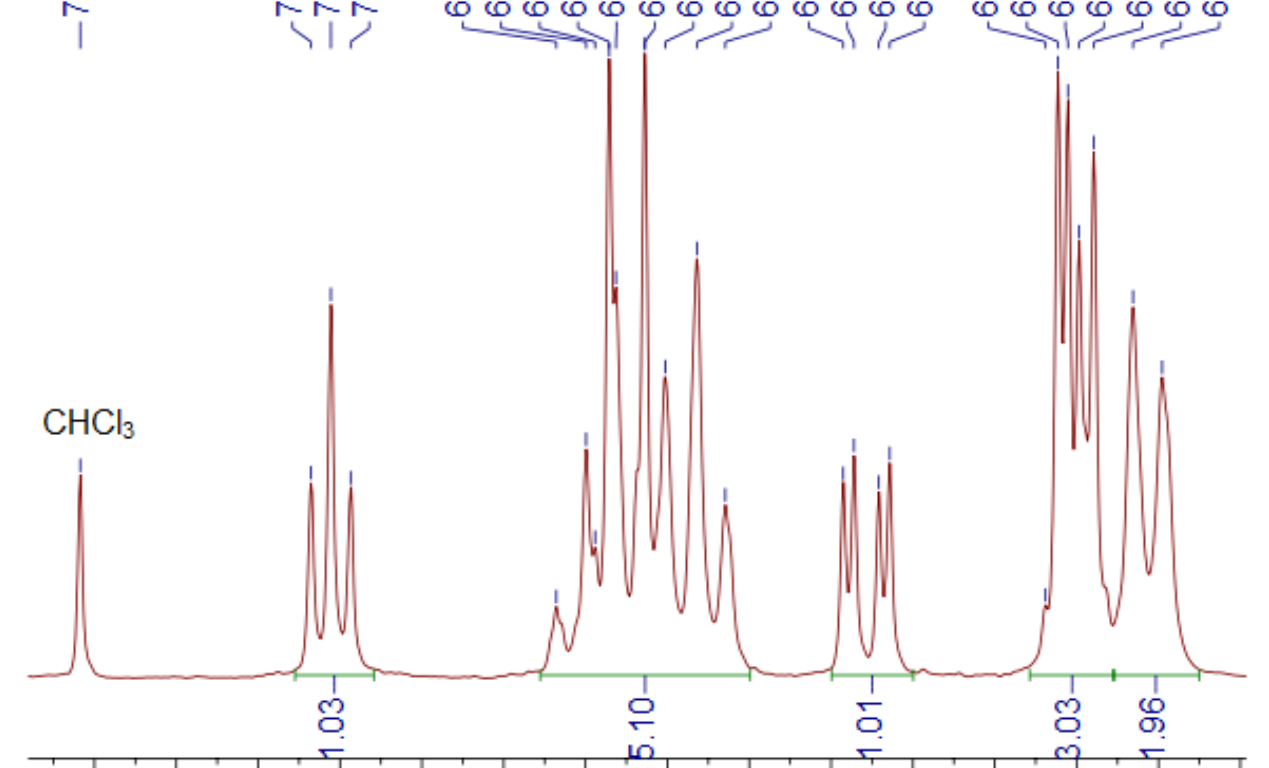

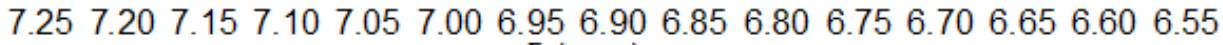
$\bar{\delta}(\mathrm{ppm})$
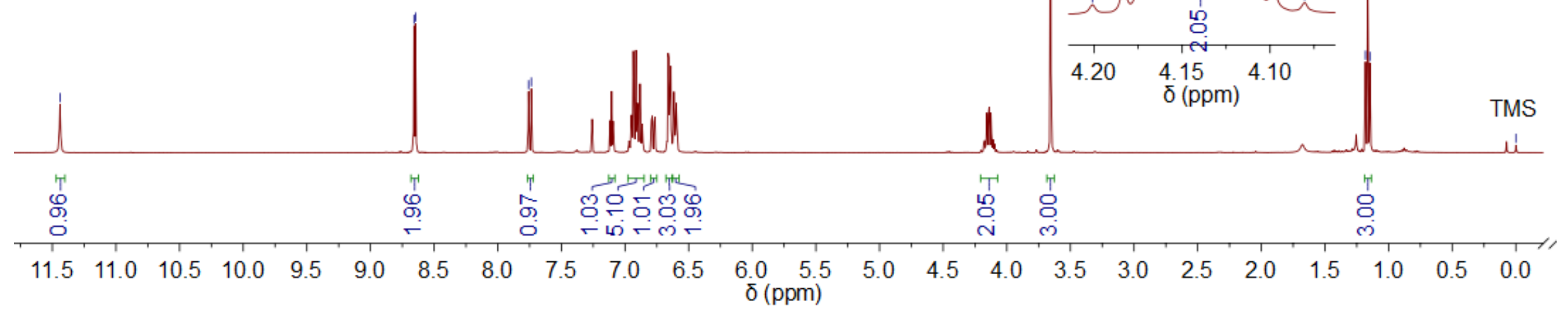
$\mathbf{5 b}-{ }^{1} \mathrm{H}$ NMR
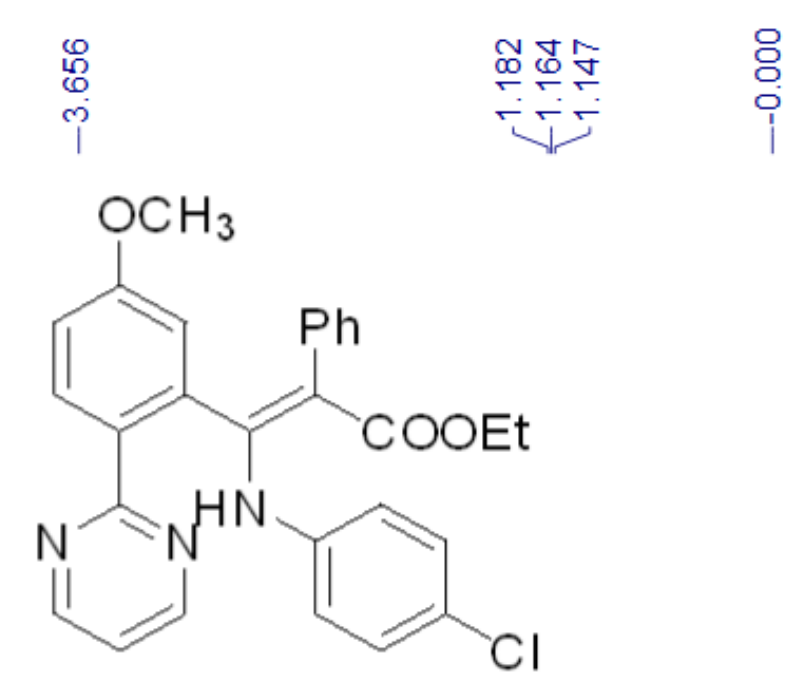

zxr454-077A-2 in $\mathrm{CDCl}_{3}$

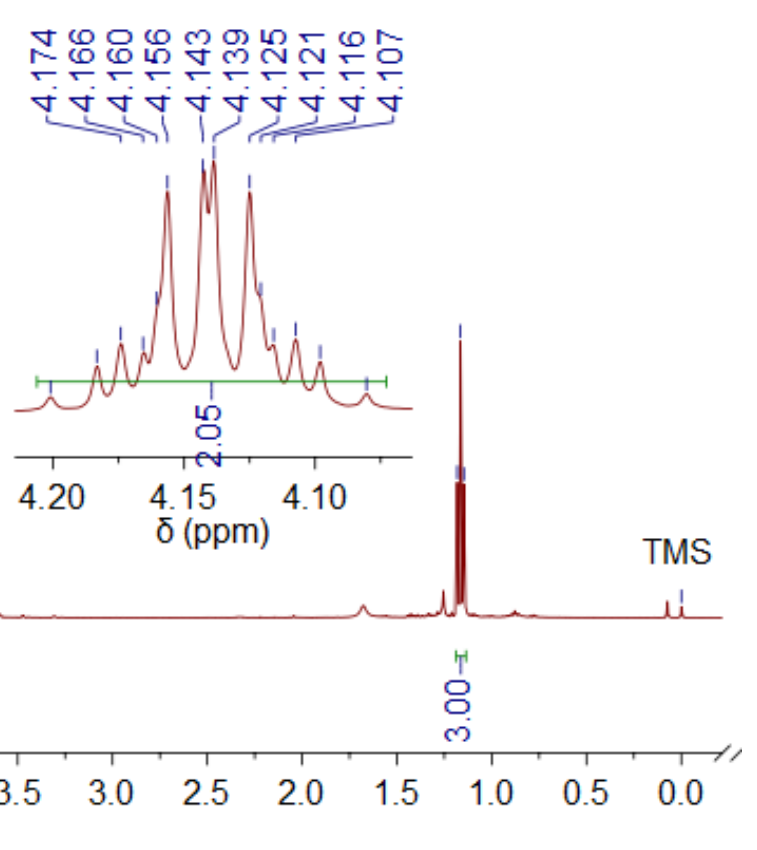



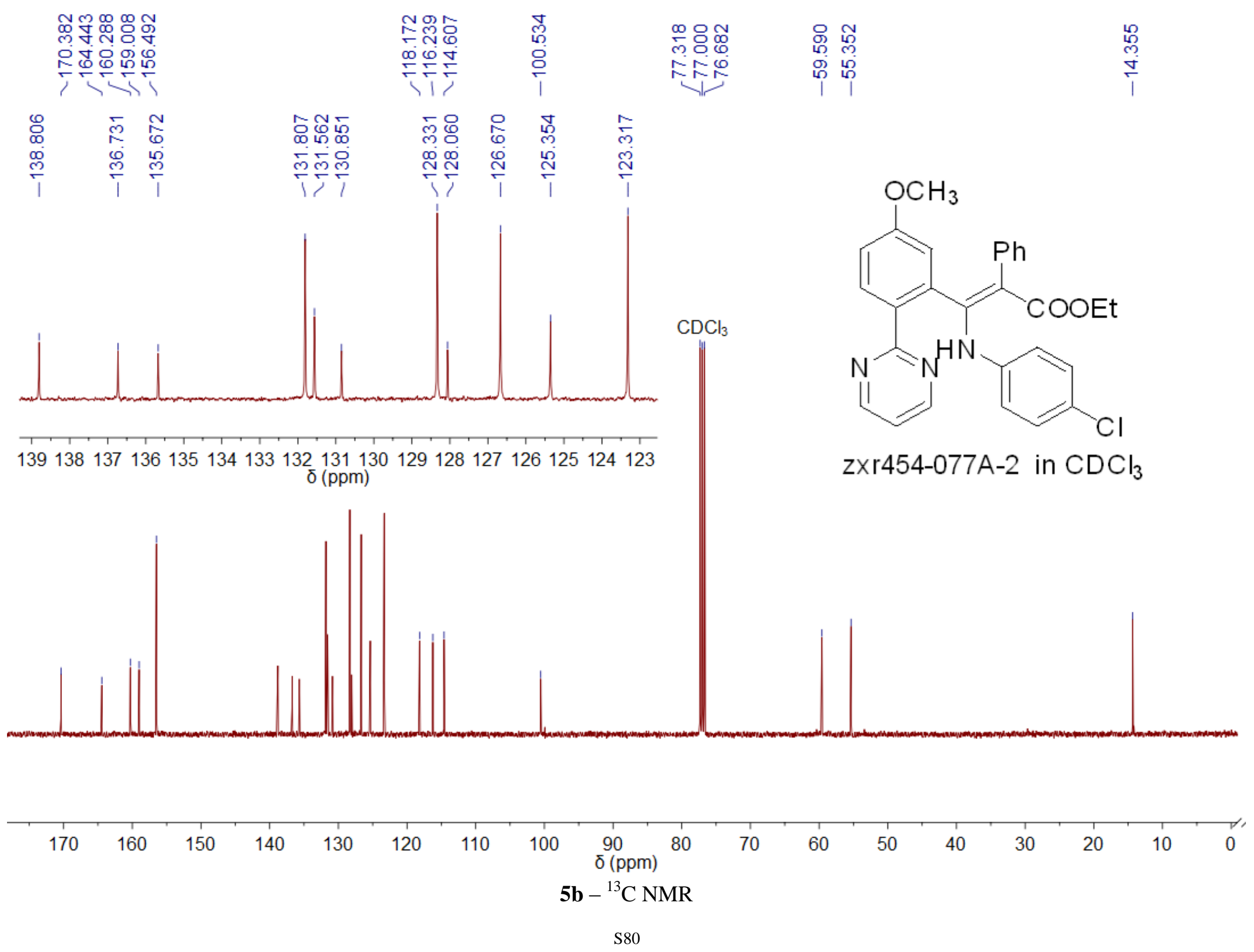


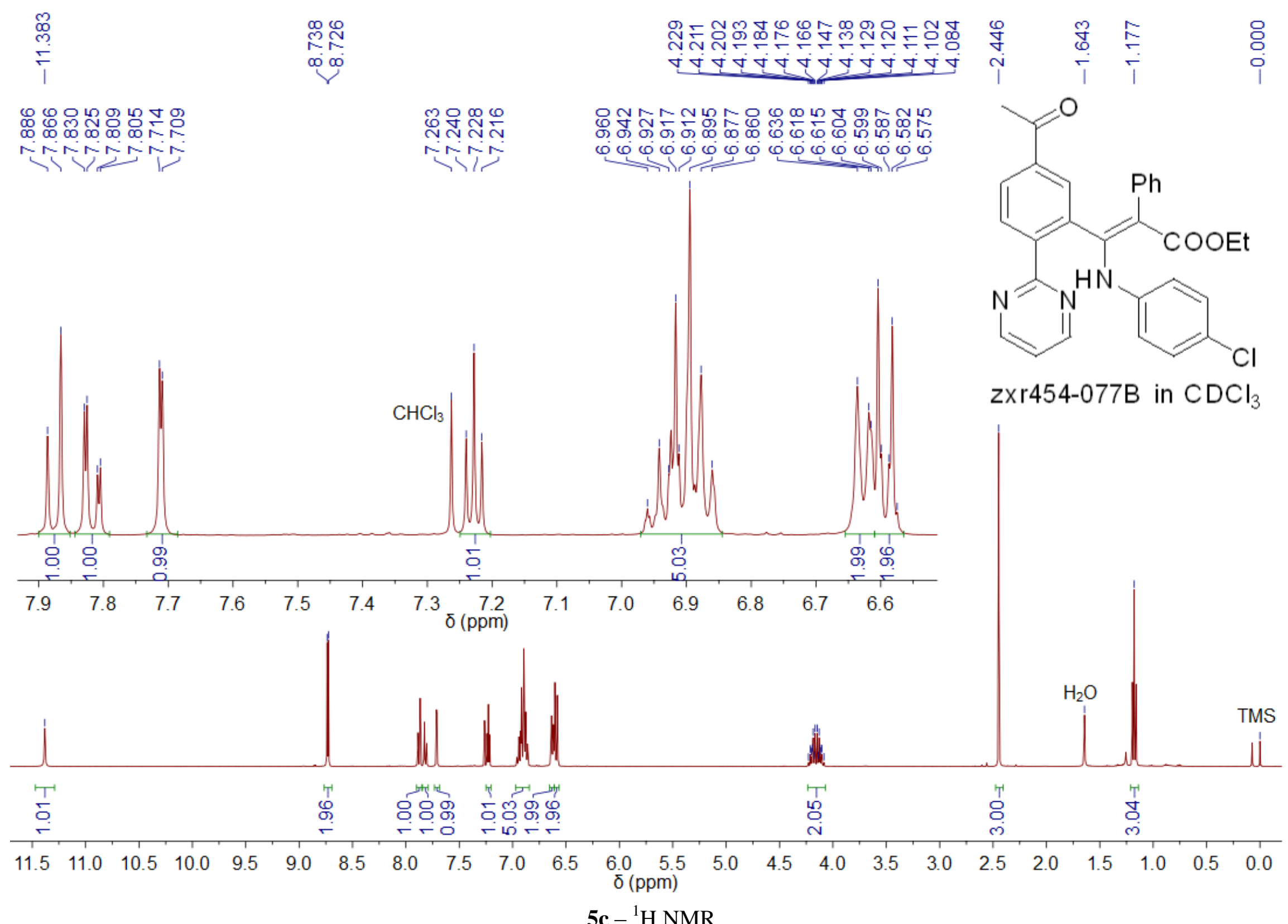



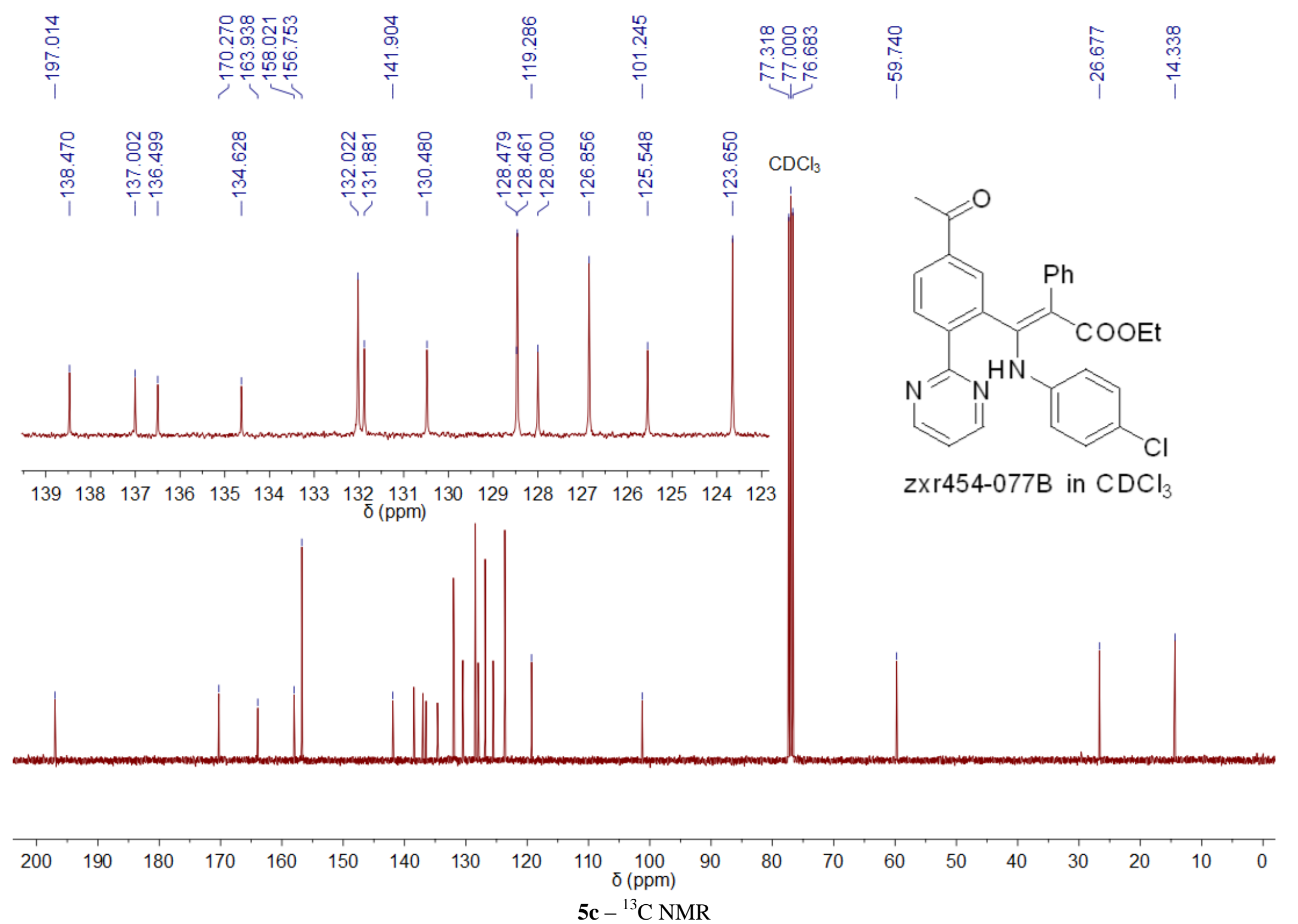


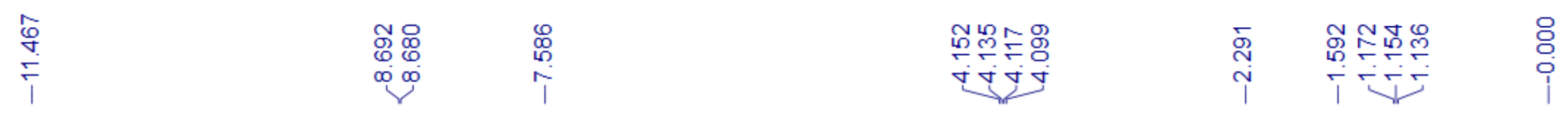

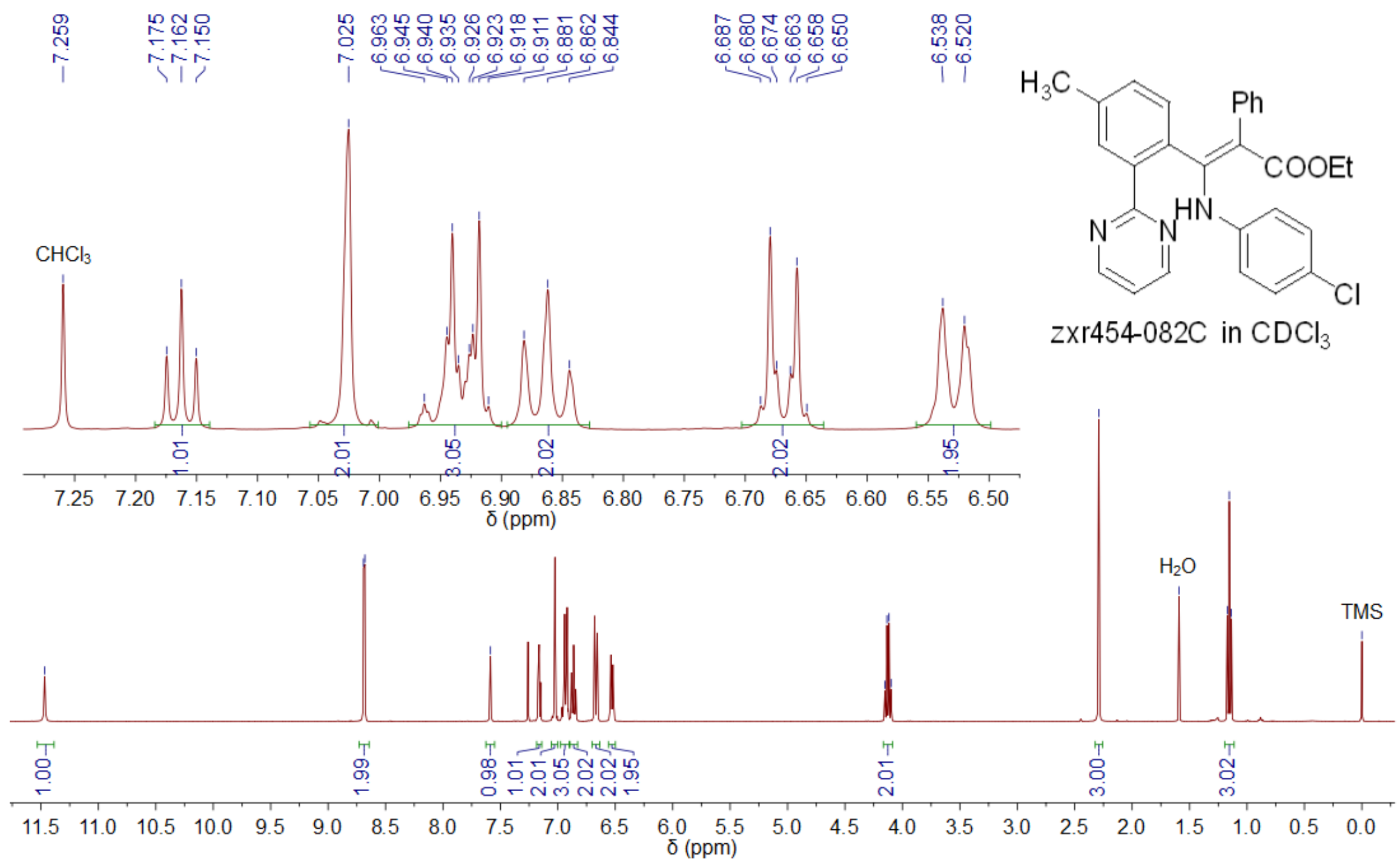

5d $-{ }^{1} \mathrm{H}$ NMR 


\begin{tabular}{|c|c|c|c|c|c|c|}
\hline 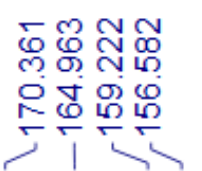 & 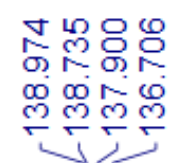 & 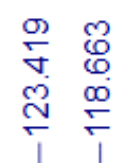 & $\begin{array}{l}\infty \\
\infty \\
0 \\
\stackrel{0}{0} \\
\end{array}$ & 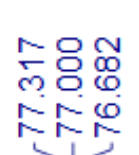 & 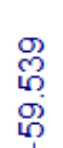 & $\frac{⿱ 亠}{\check{r}}$ \\
\hline
\end{tabular}

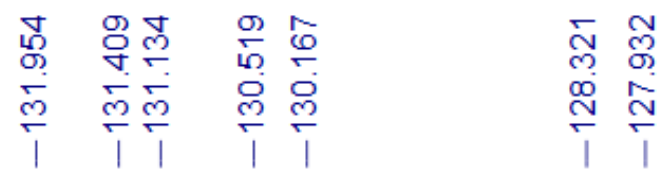

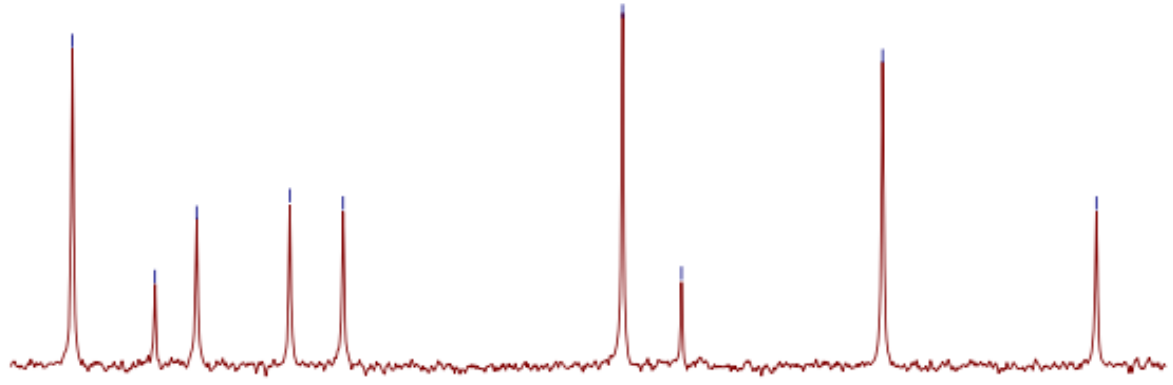

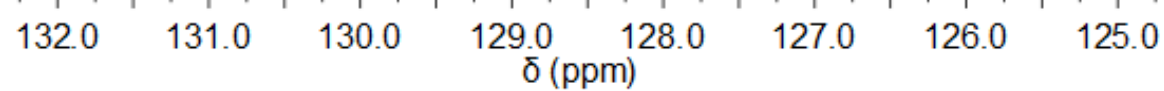

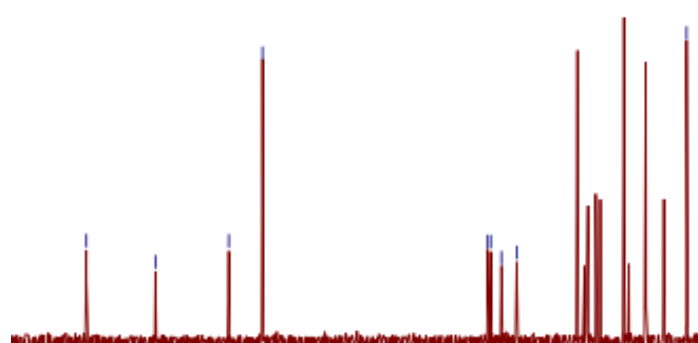

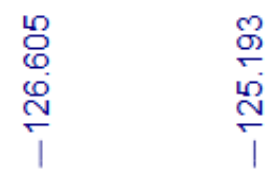

$\mathrm{CDCl}_{3}$

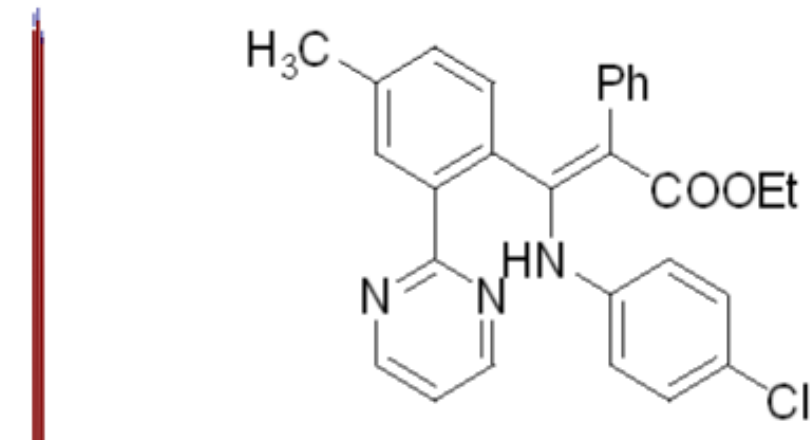

zxr454-082C in $\mathrm{CDCl}_{3}$

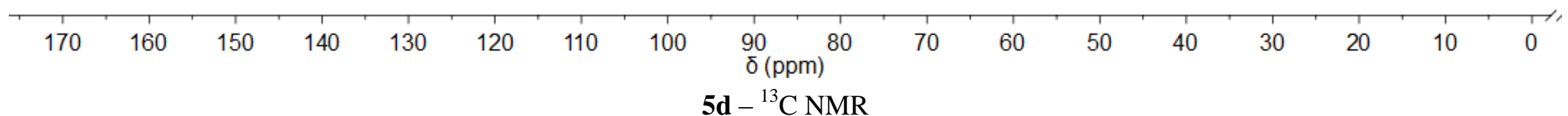




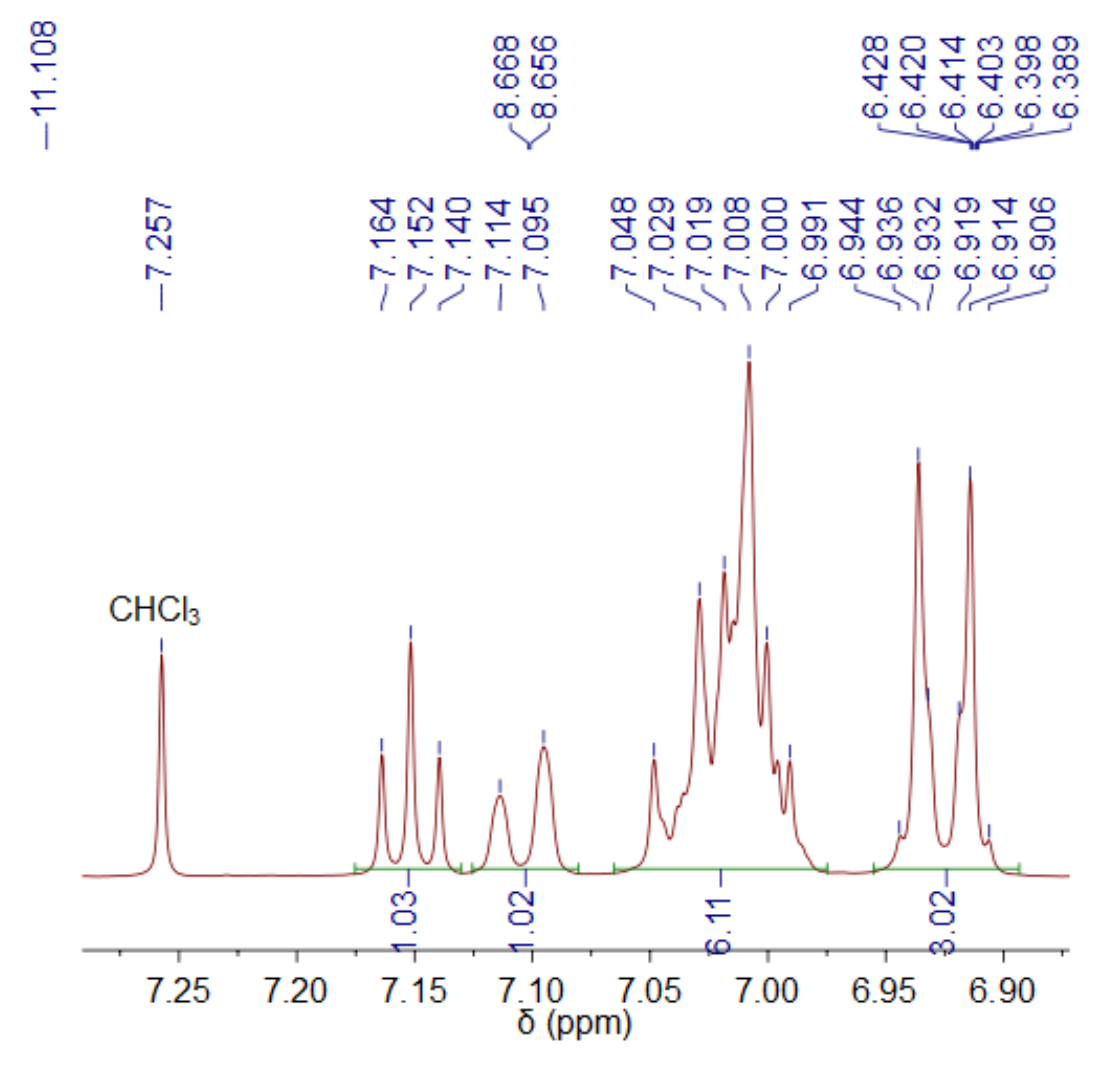

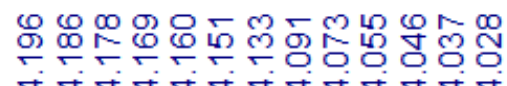

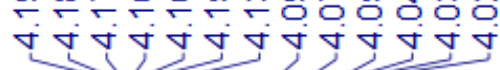

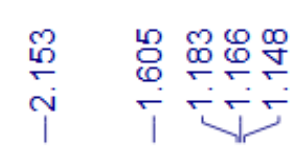

웅
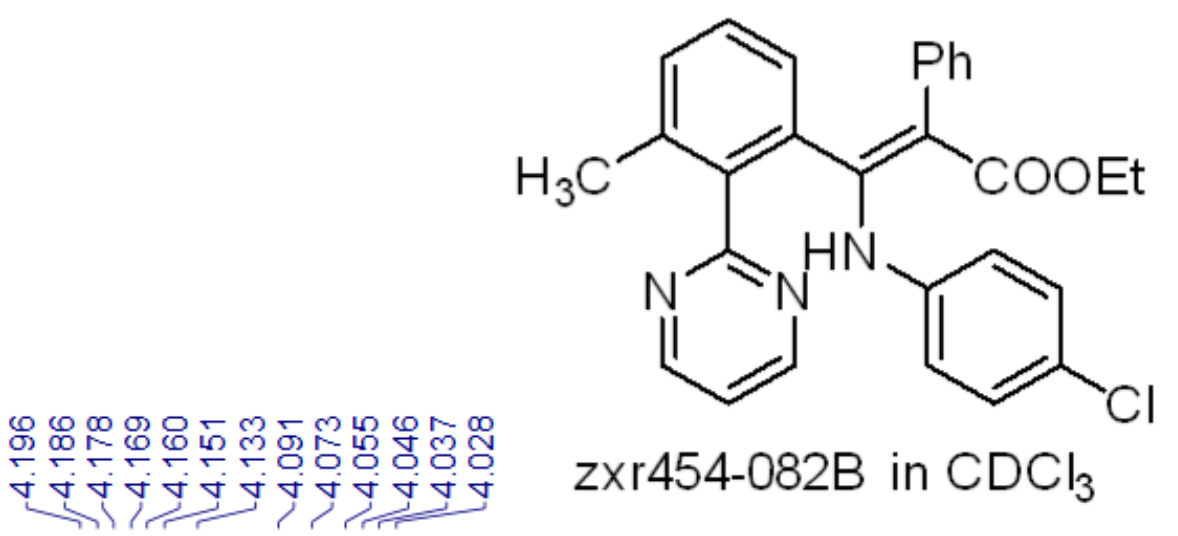
zxr454-082B in $\mathrm{CDCl}_{3}$
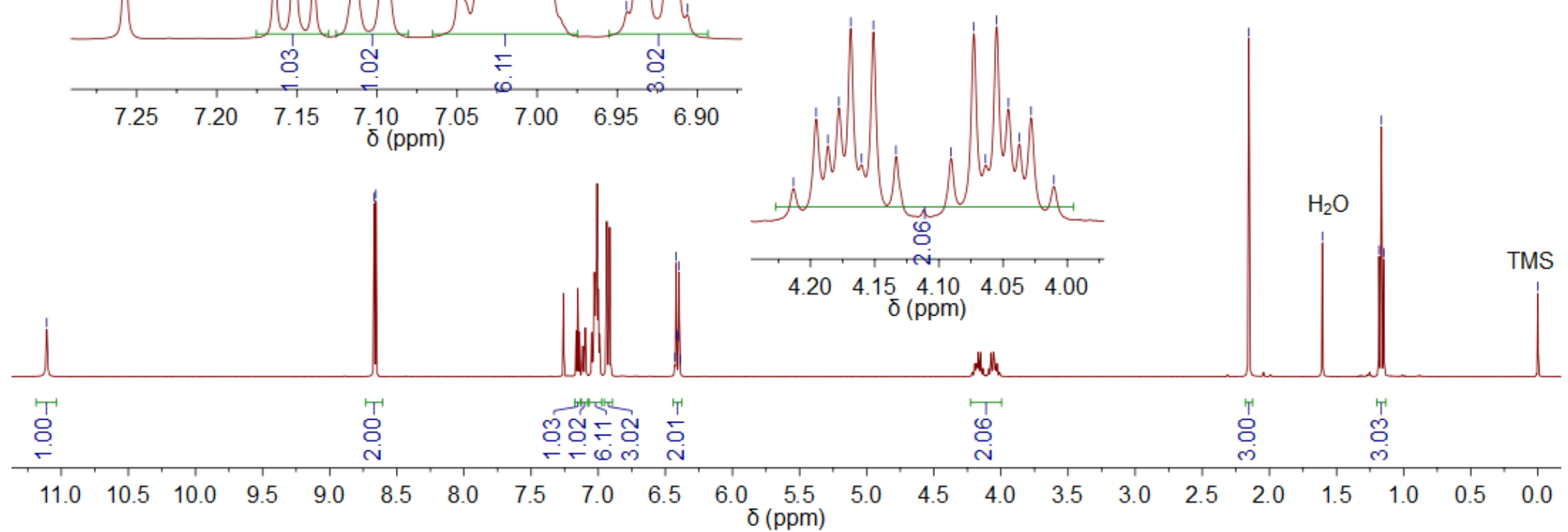

5e $-{ }^{1} \mathrm{H}$ NMR 

品 觉 它员
인
일

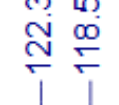

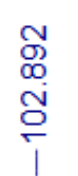

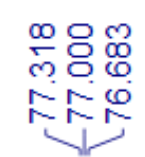

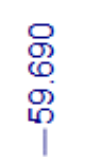
$\underset{\substack{0 \\ 0}}{\substack{\text { N } \\ 0}}$

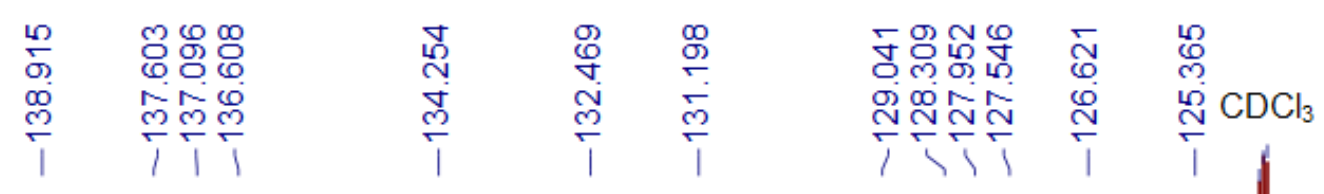

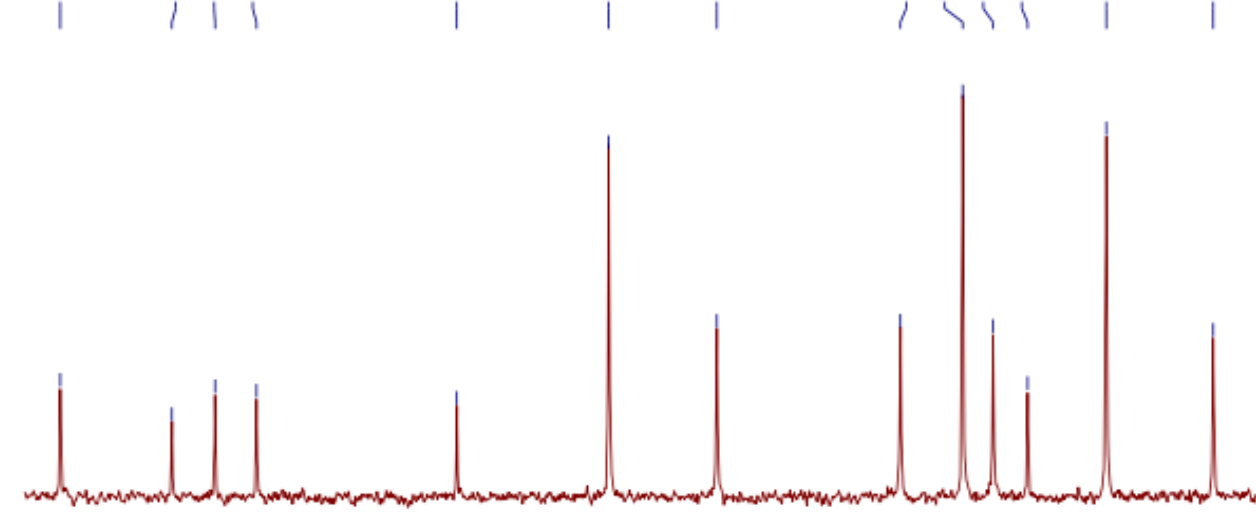

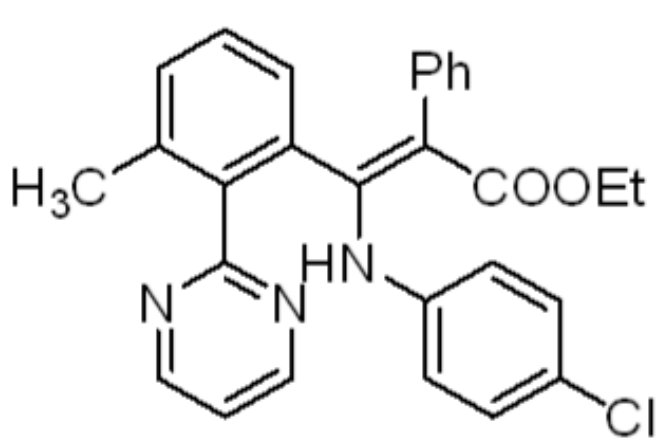

zxr454-082B in $\mathrm{CDCl}_{3}$
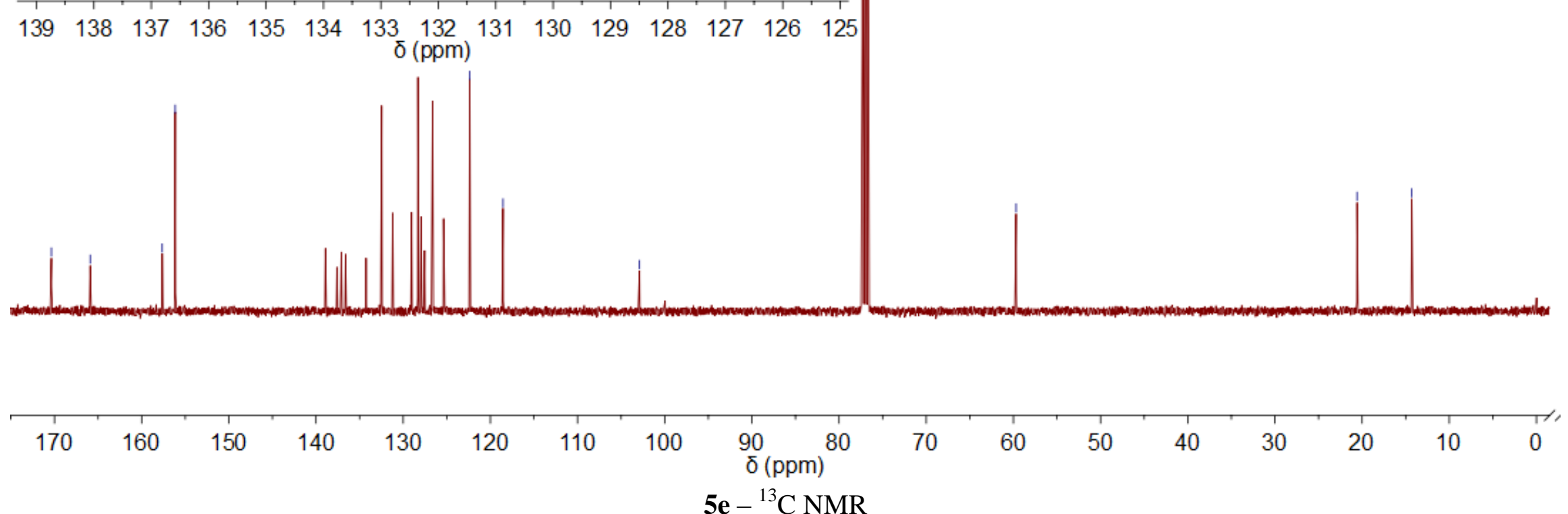


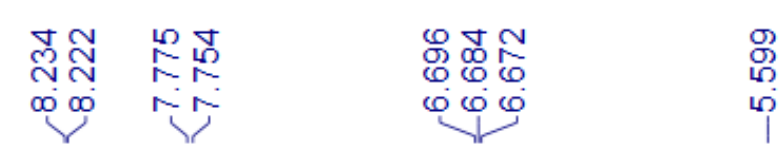

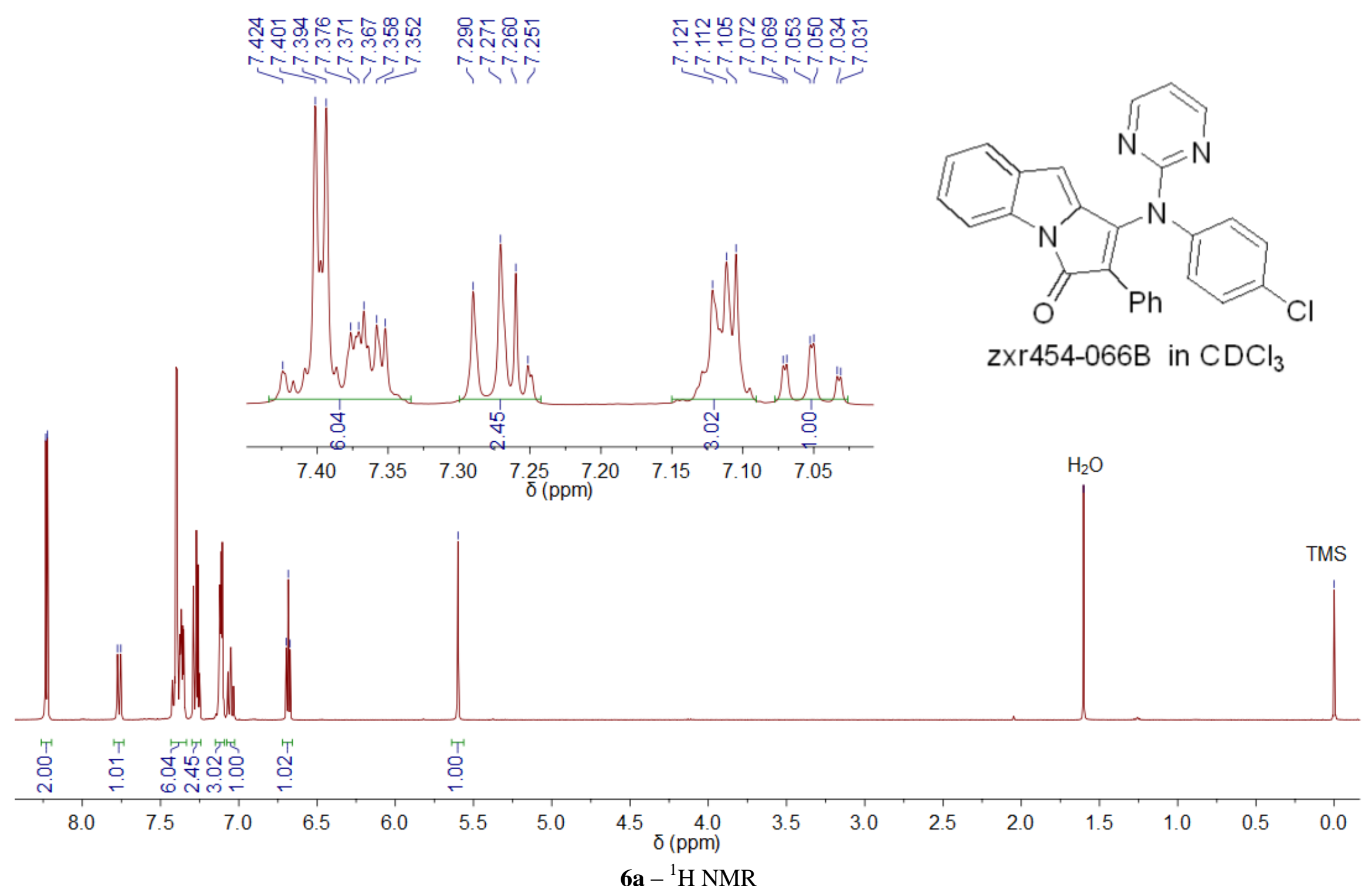



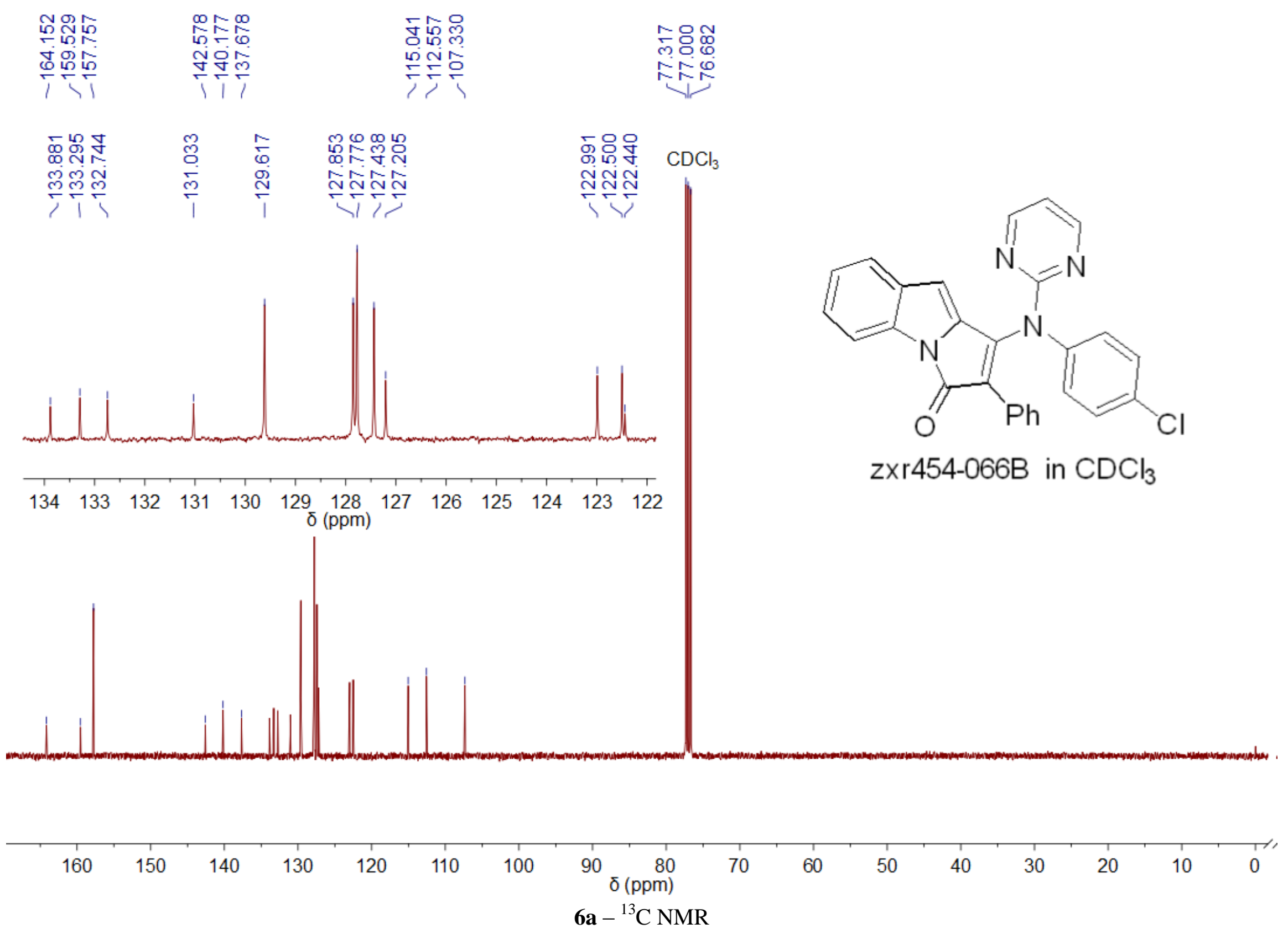


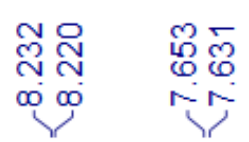

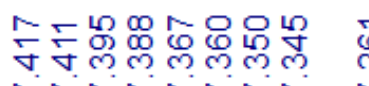

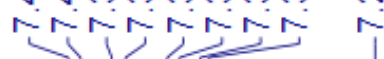

윰묻응용용

证年

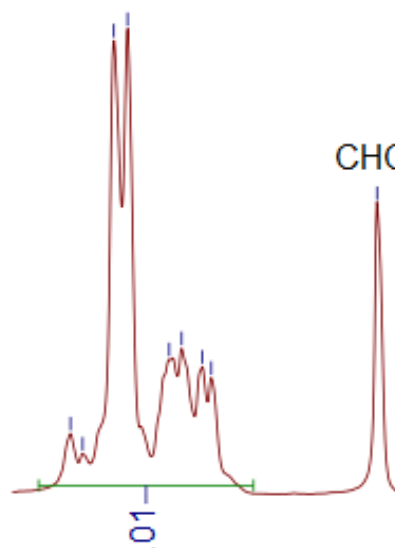

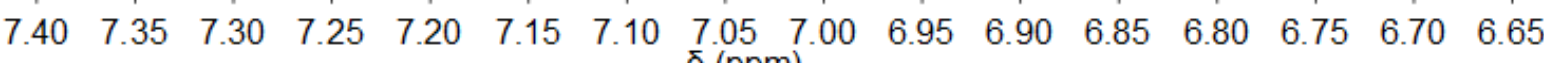
$\bar{\delta}(\mathrm{ppm})$

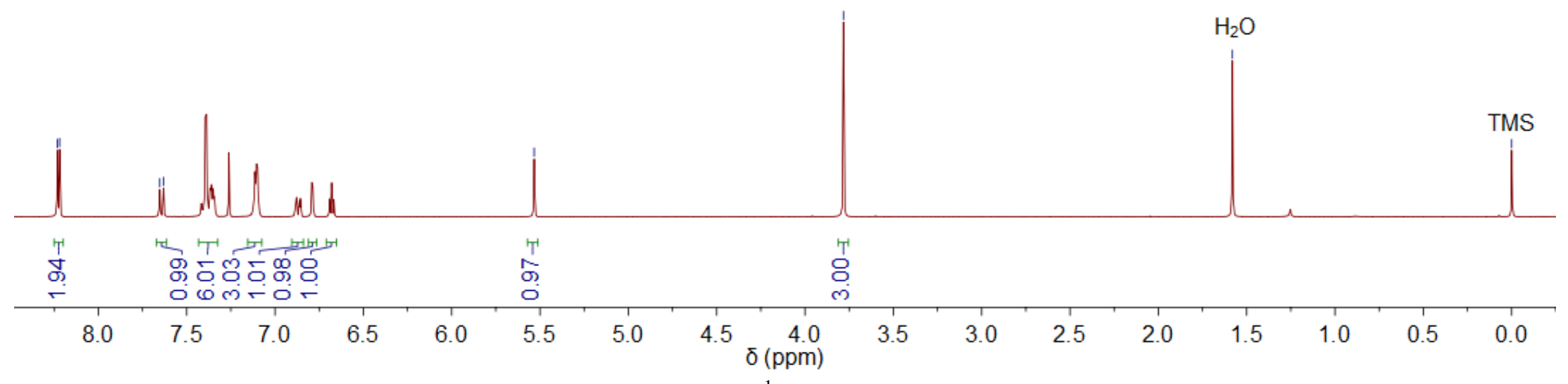

6b $-{ }^{1} \mathrm{H}$ NMR

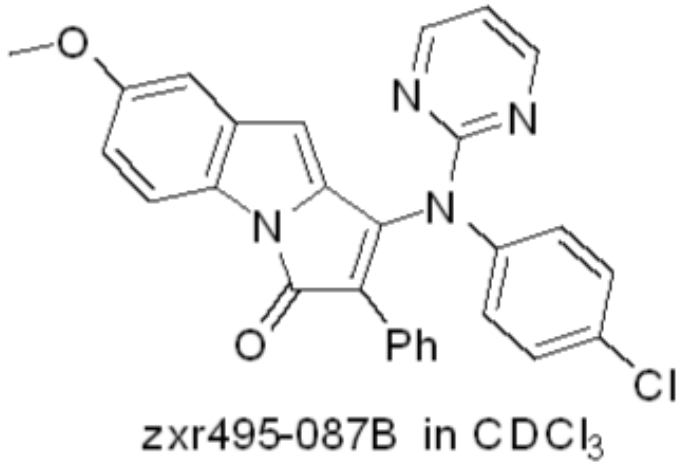

zxr495-087B in $\mathrm{CDCl}_{3}$
0

ต
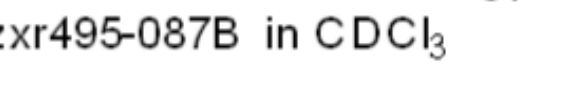


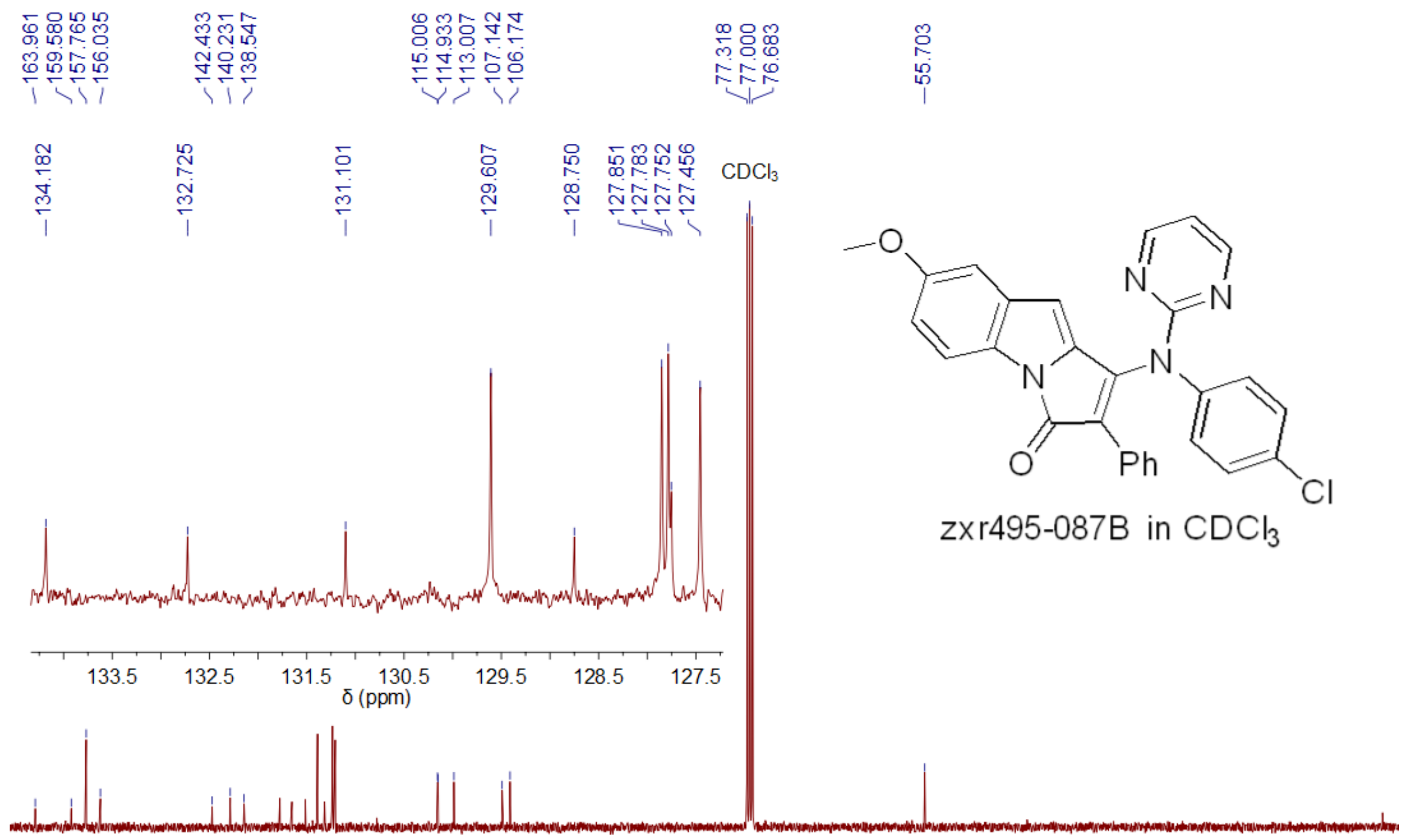

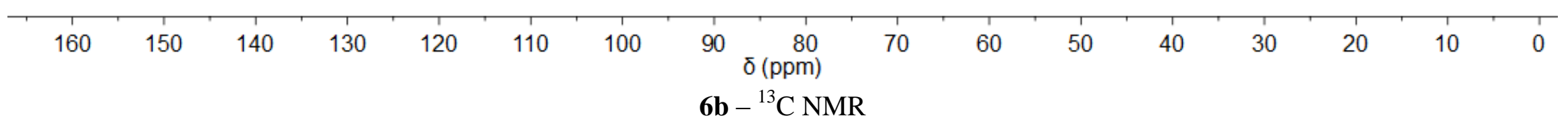




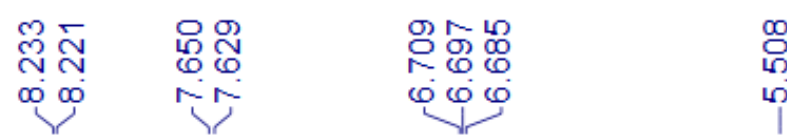

0
0
0
0

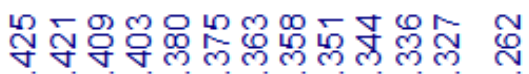

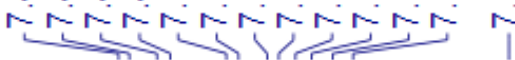

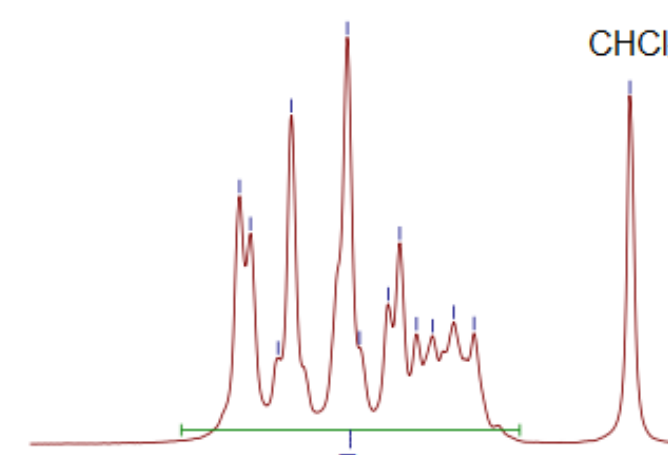

$\mathrm{CHCl}_{3}$

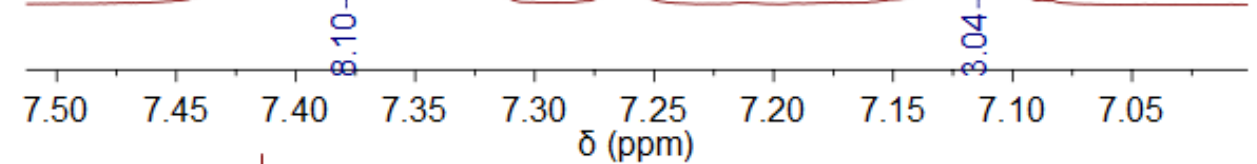

zxr454-100B in $\mathrm{CDCl}_{3}$

$\frac{1}{20}$
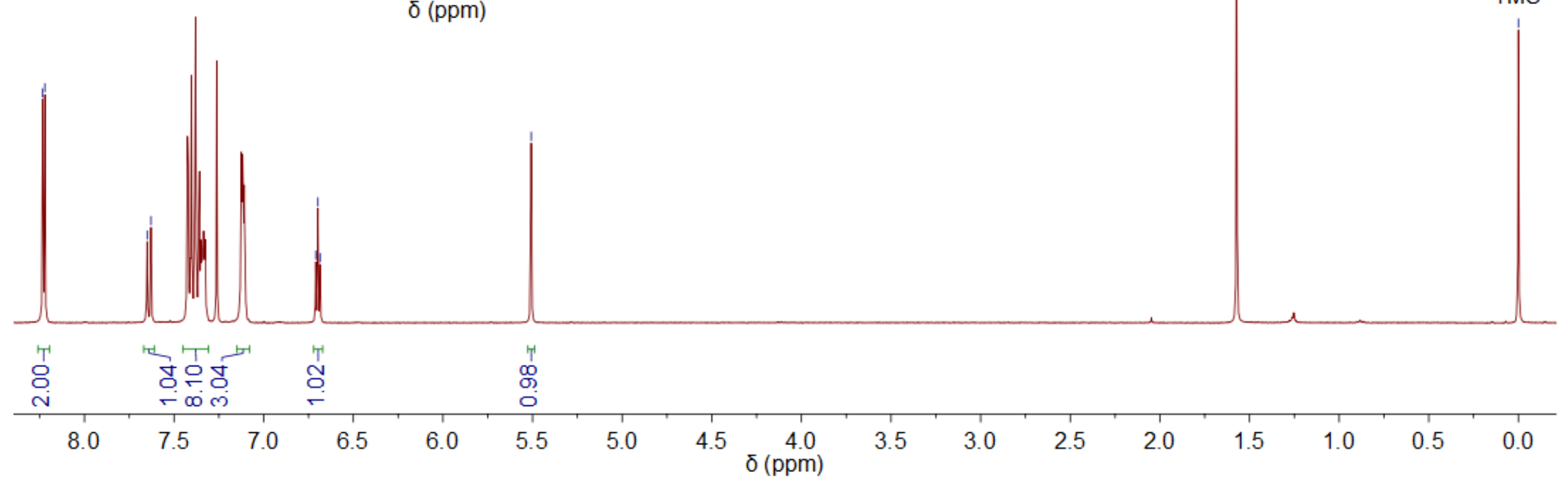

6c $-{ }^{1} \mathrm{H}$ NMR 


\begin{tabular}{|c|c|}
\hline 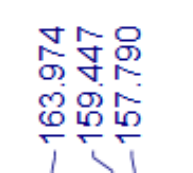 & 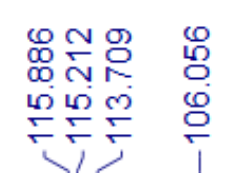 \\
\hline
\end{tabular}

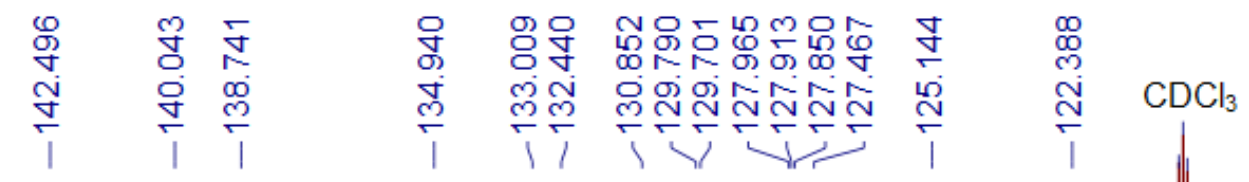

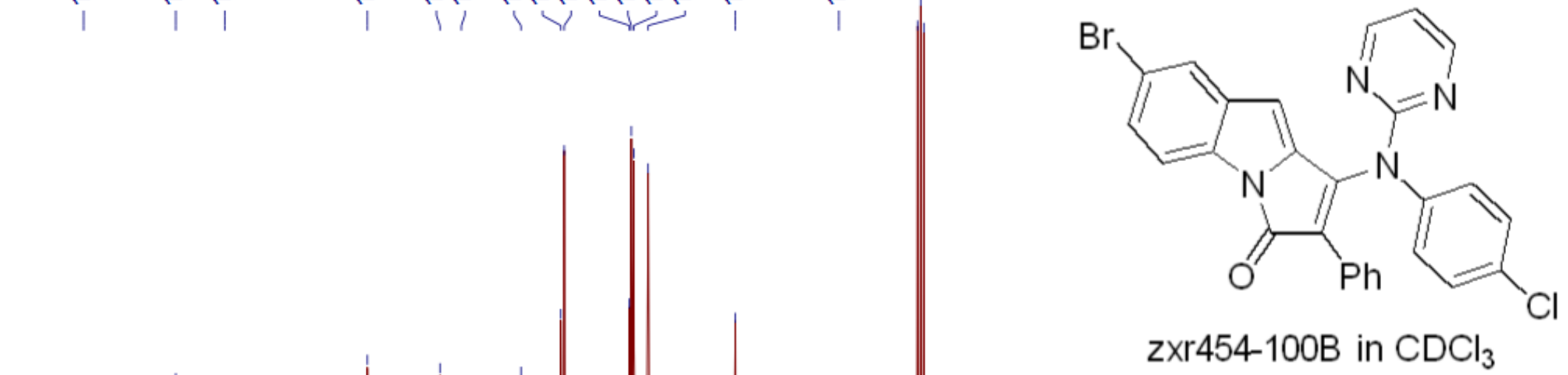

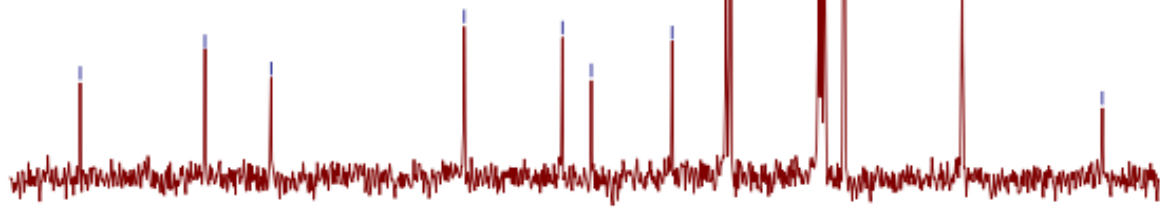
zxr454-100B in $\mathrm{CDCl}_{3}$

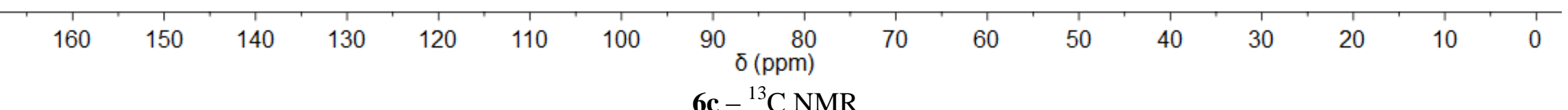


원

$\infty \infty \quad \sim-m i n$

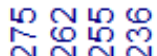

$\sim 20$

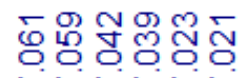

rinis

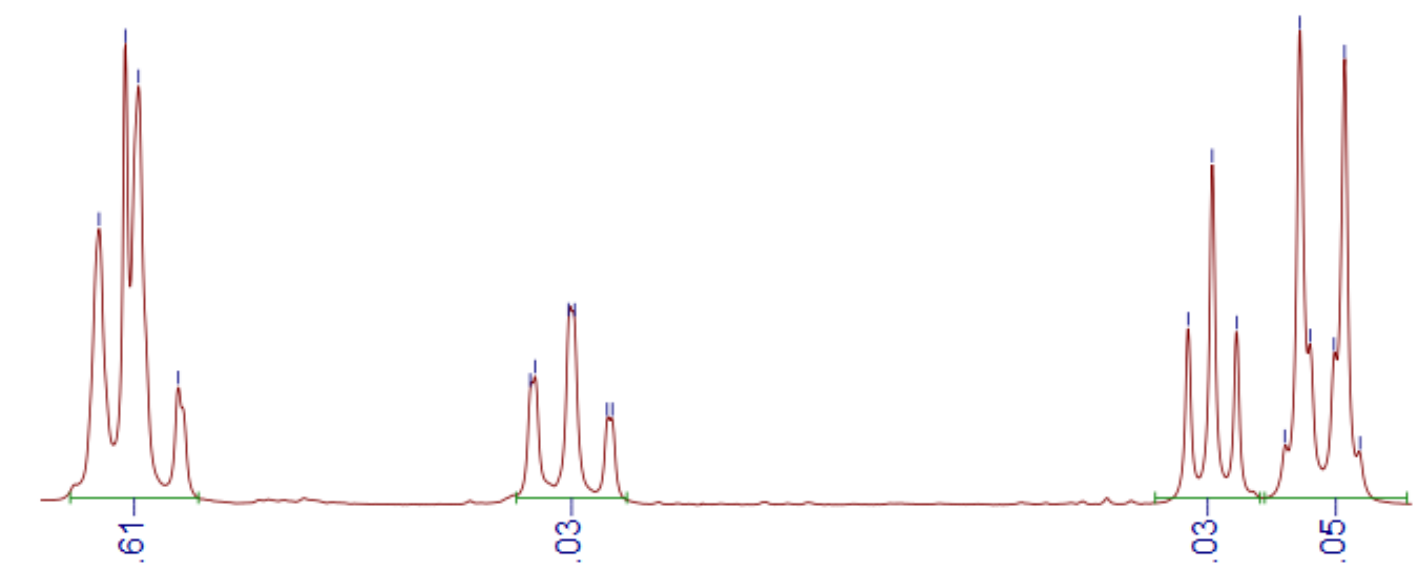

$\begin{array}{lllllllllllllll}7.30 & 7.25 & 7.20 & 7.15 & 7.10 & 7.05 & 7.00 & 6.95 & 6.90 & 6.85 & 6.80 & 6.75 & 6.70 & 6.65\end{array}$
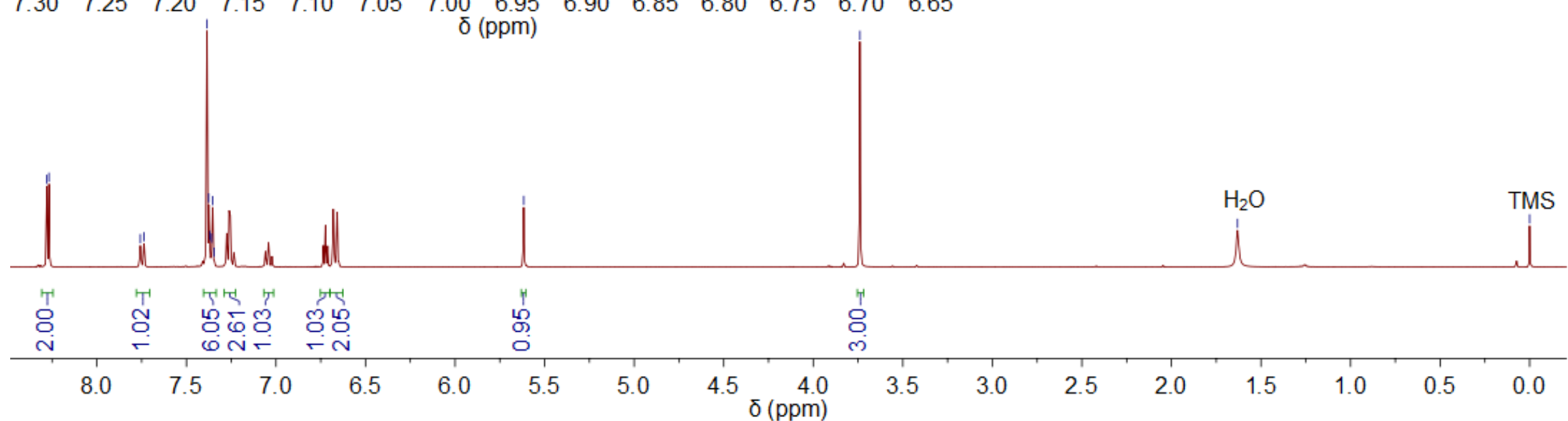

6d $-{ }^{1} \mathrm{H}$ NMR

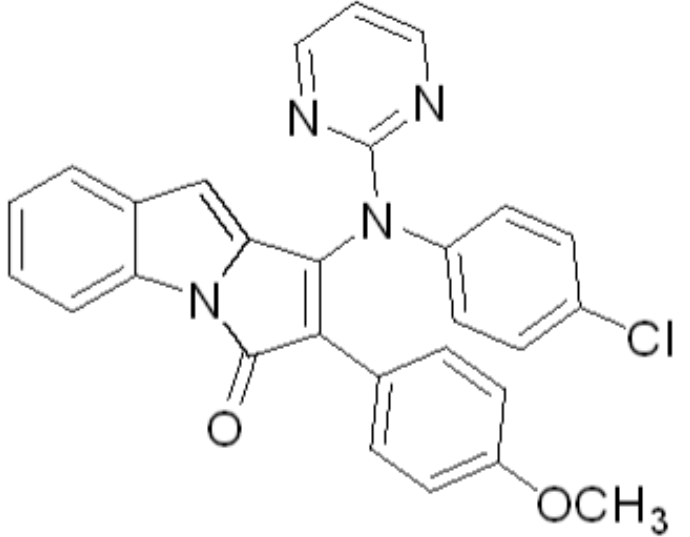

fzl495-083 in $\mathrm{CDCl}_{3}$ 


\begin{tabular}{|c|c|c|}
\hline 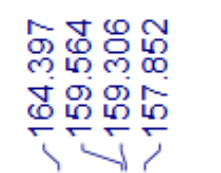 & 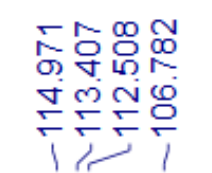 & 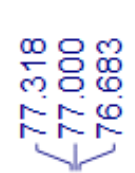 \\
\hline
\end{tabular}

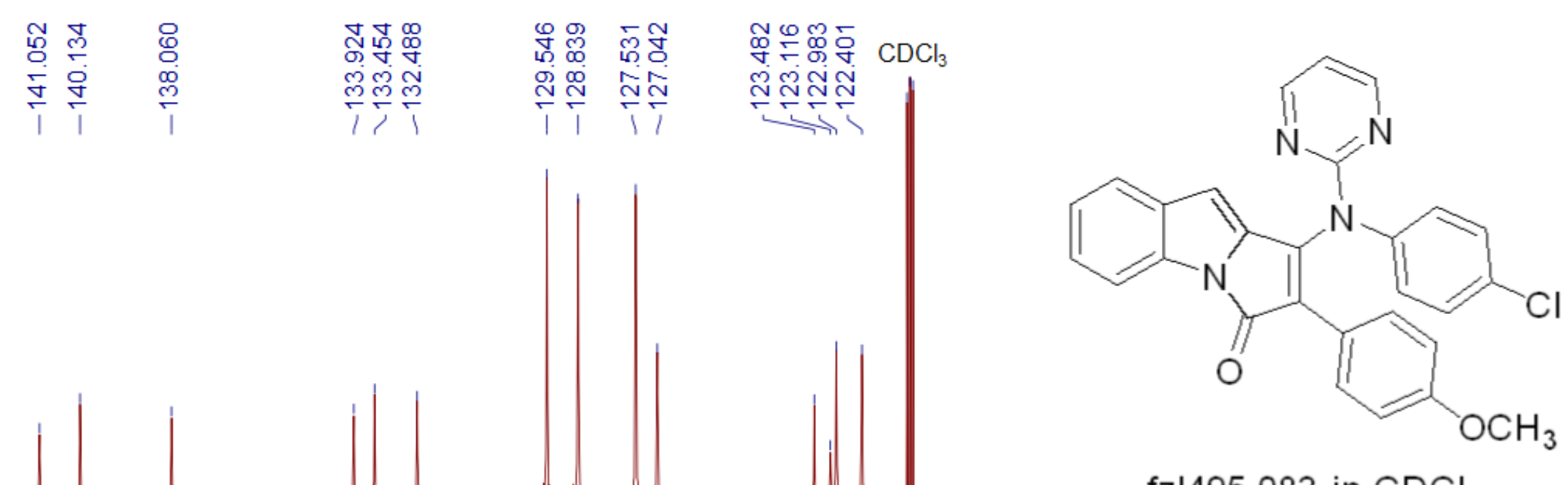

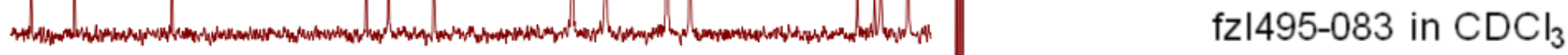
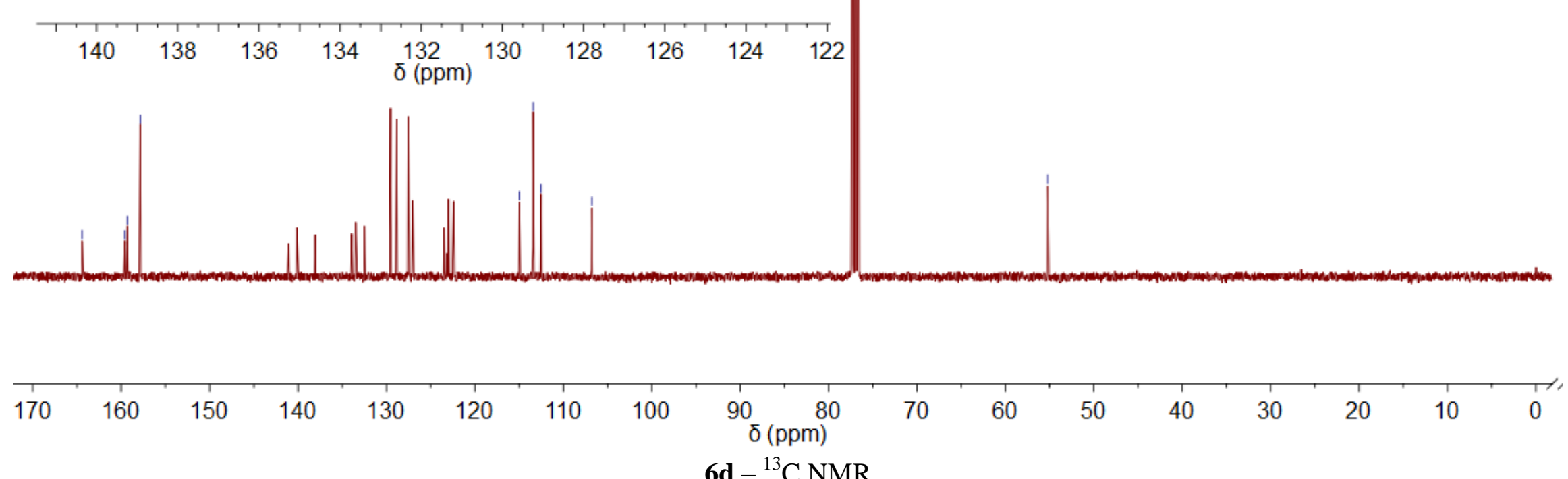


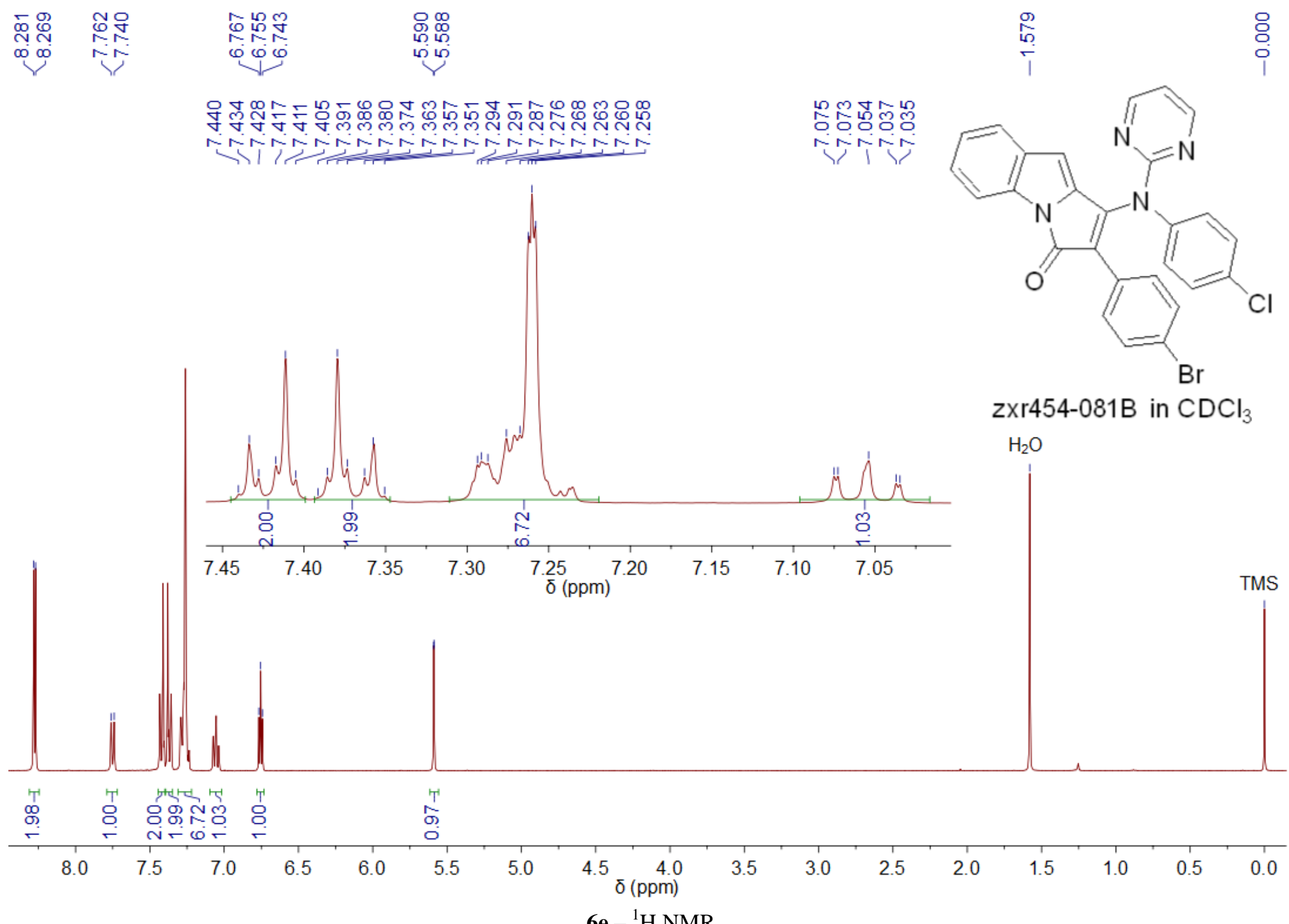

6e $-{ }^{1} \mathrm{H}$ NMR 


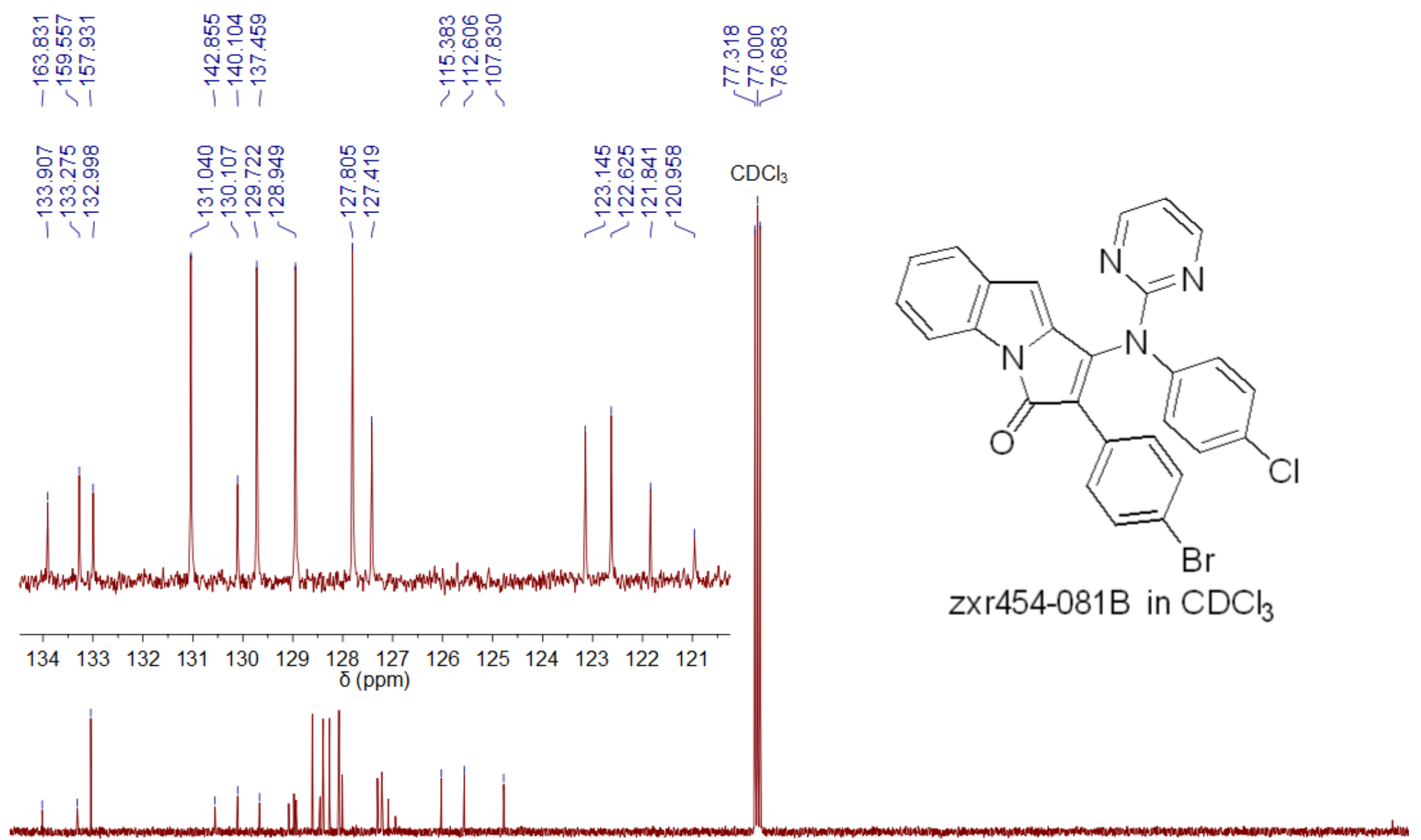

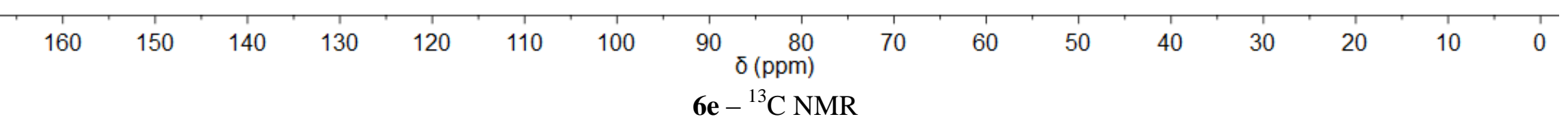




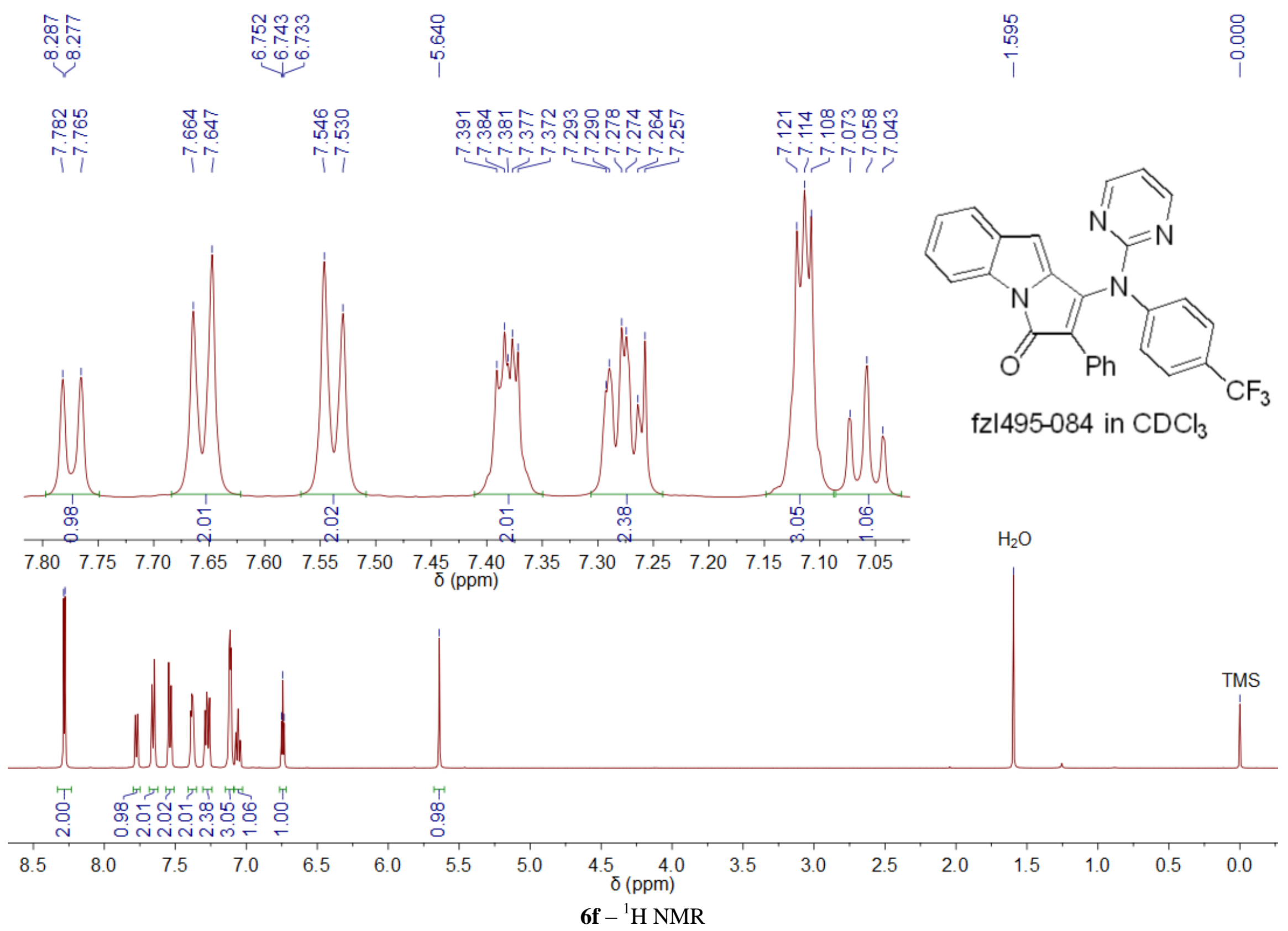



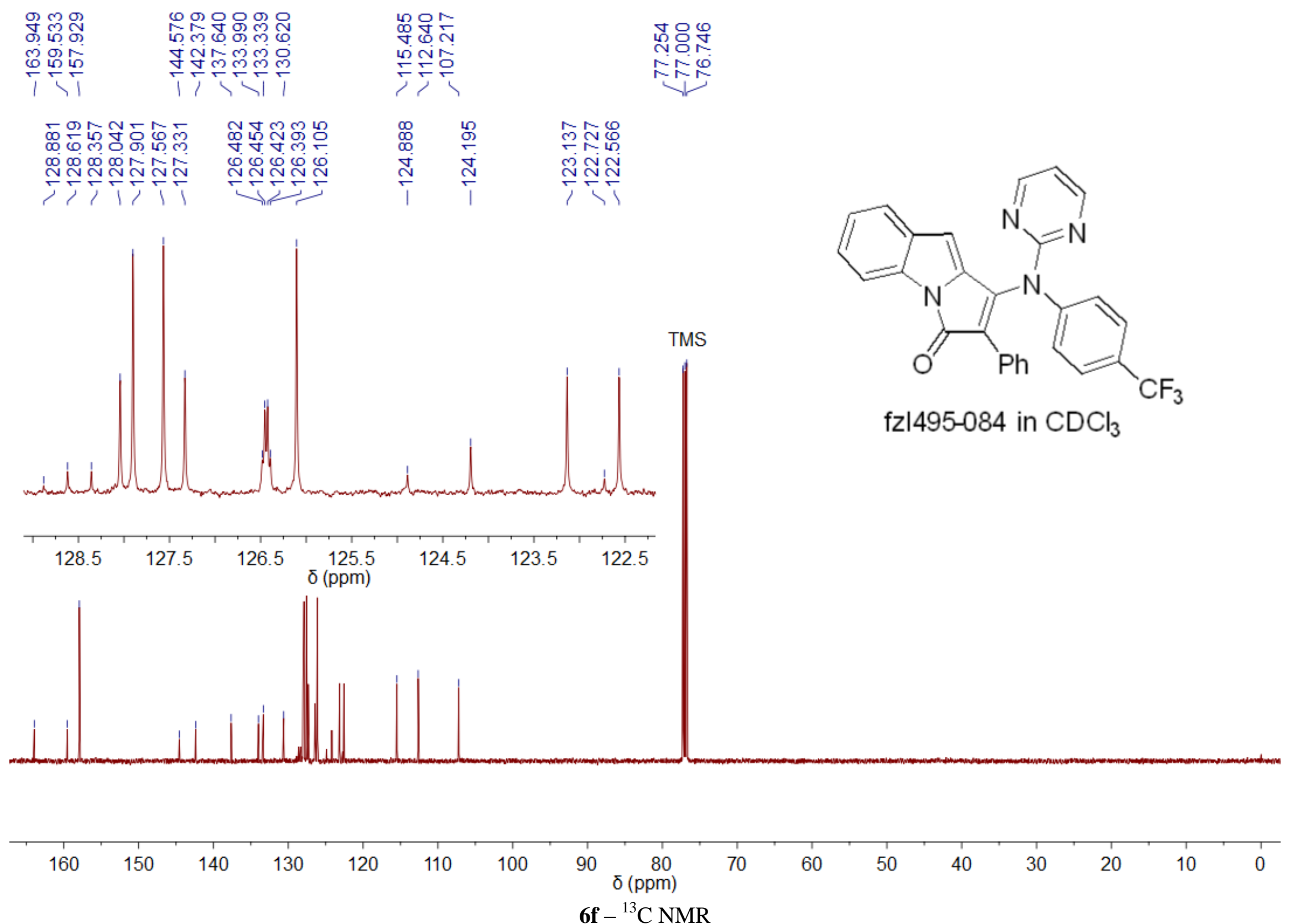


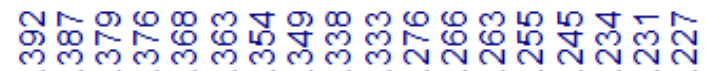

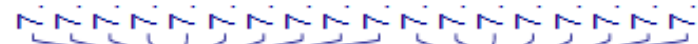

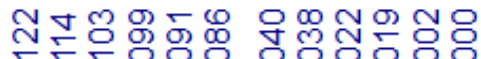
证牙证
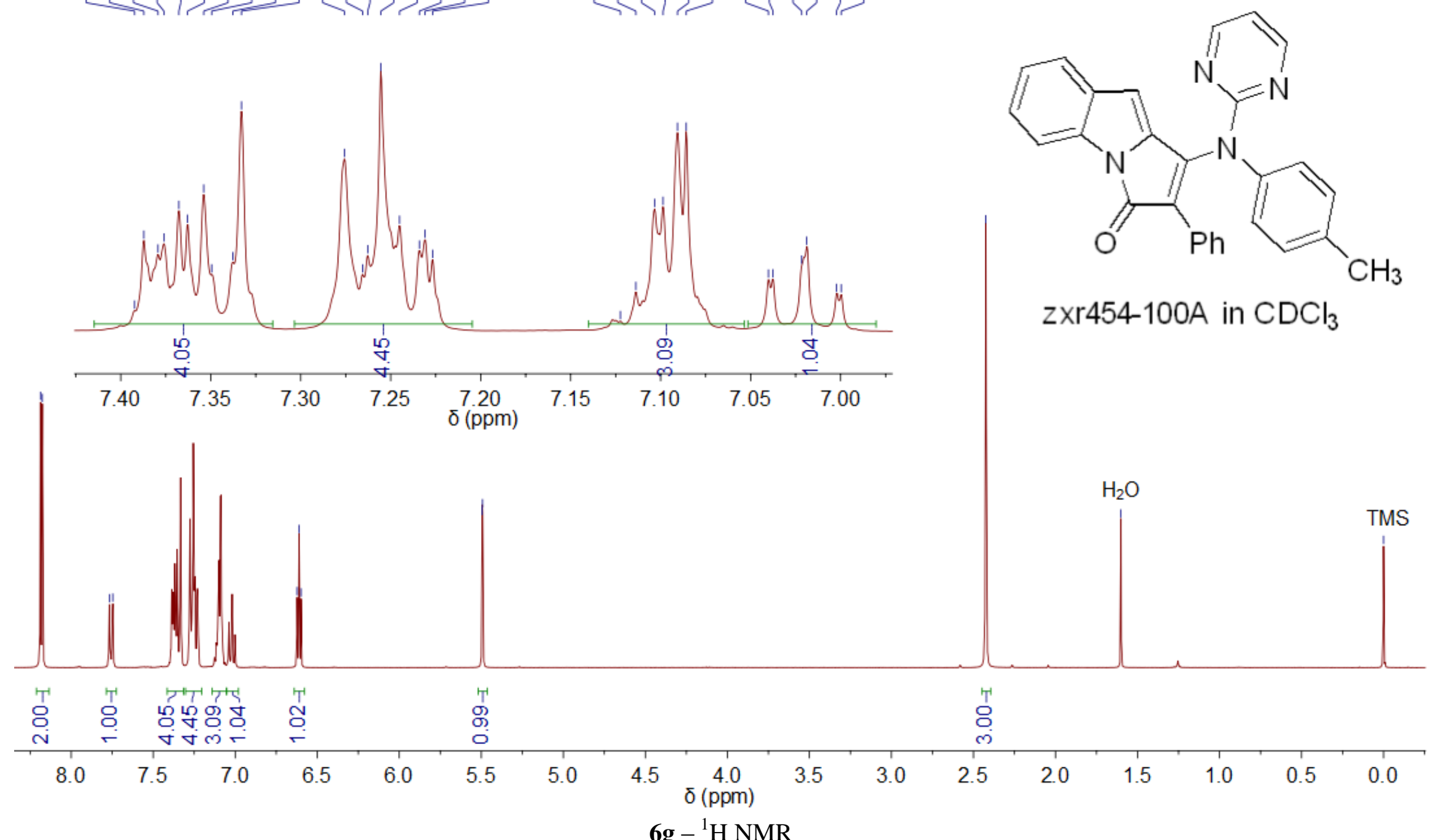

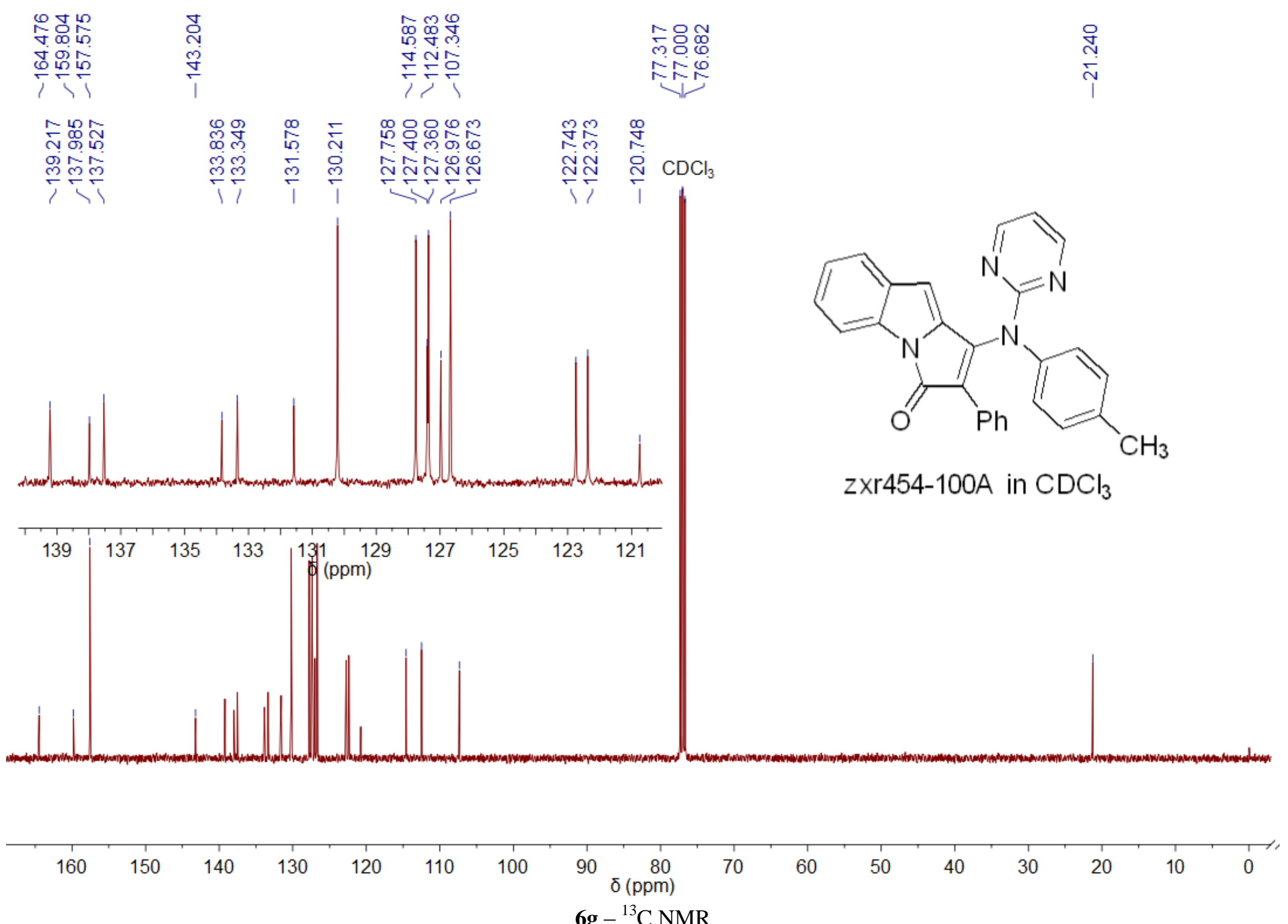

6g $-{ }^{13} \mathrm{C}$ NMR 

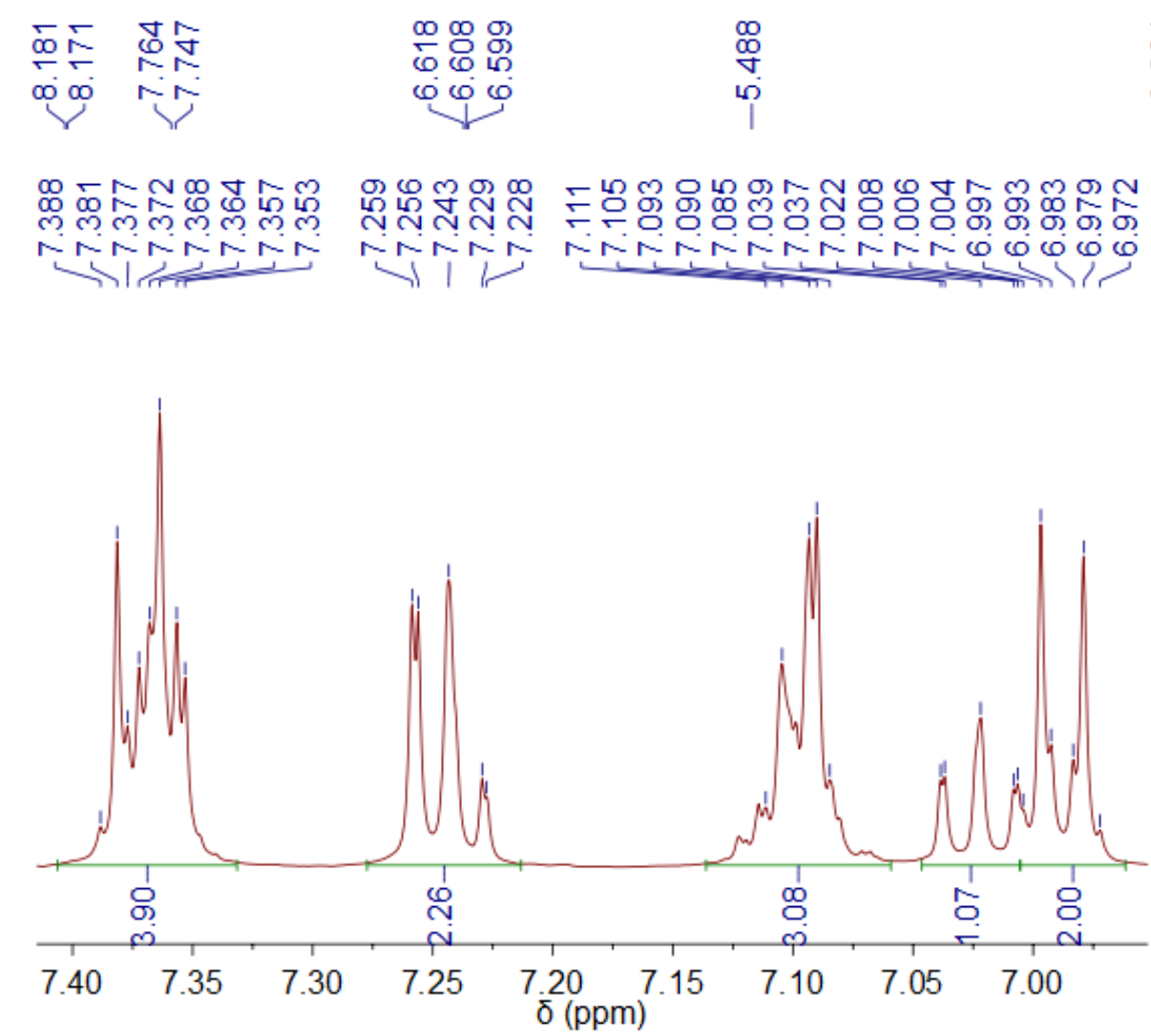

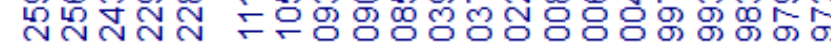

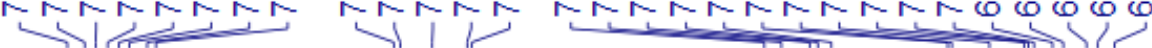
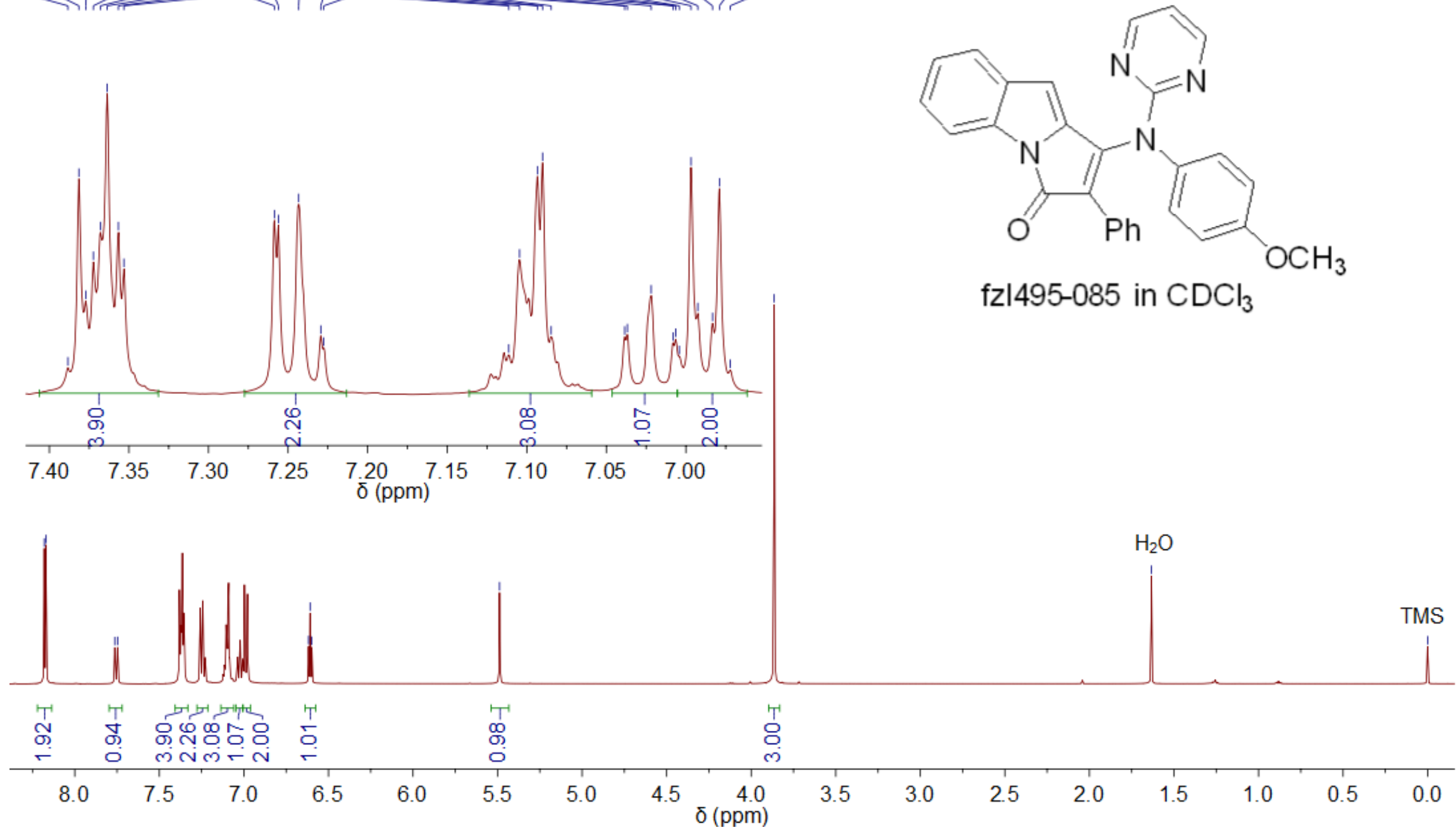

6h $-{ }^{1} \mathrm{H}$ NMR 

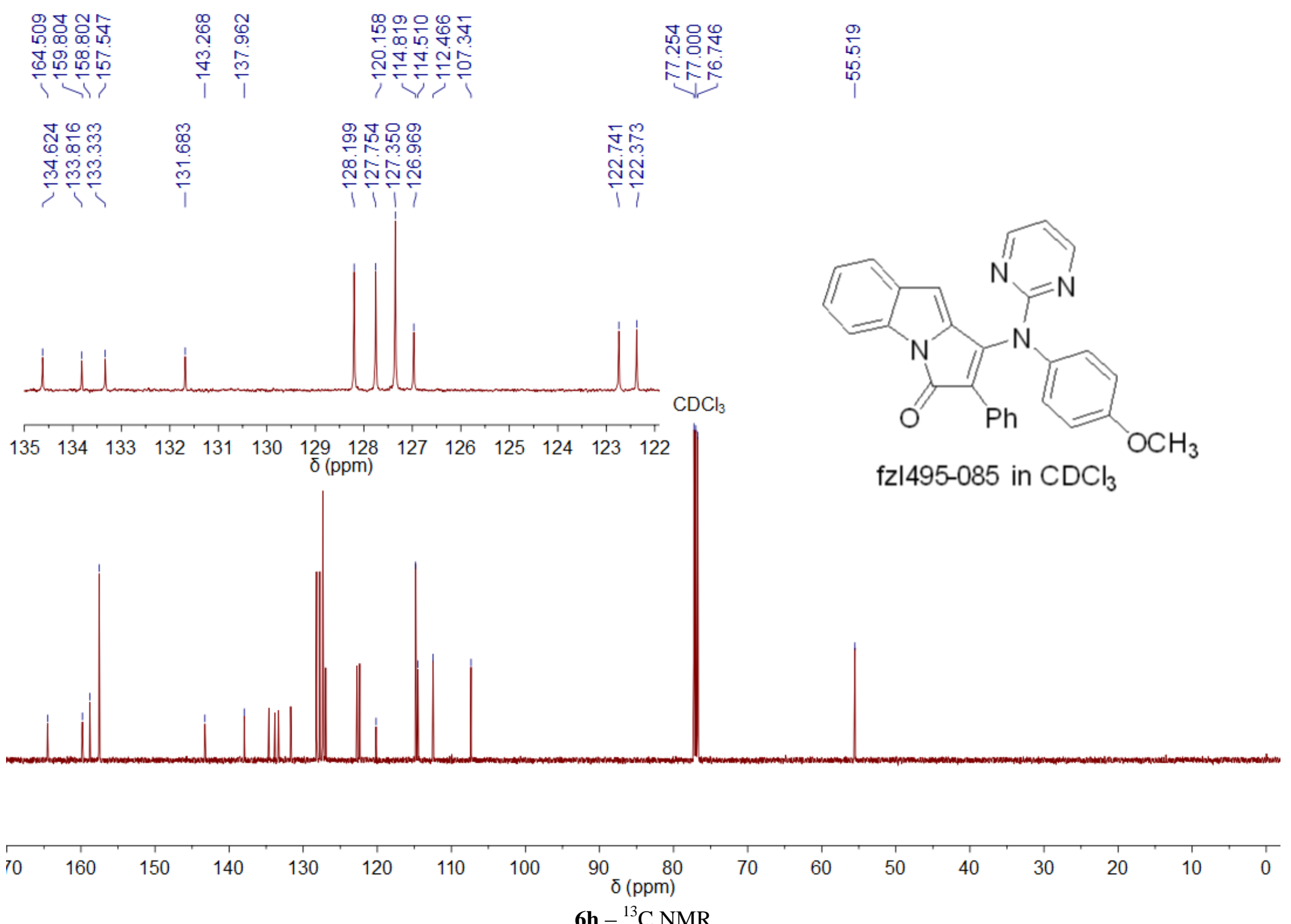

6h $-{ }^{13} \mathrm{C}$ NMR 


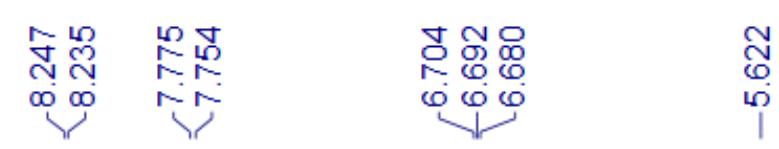

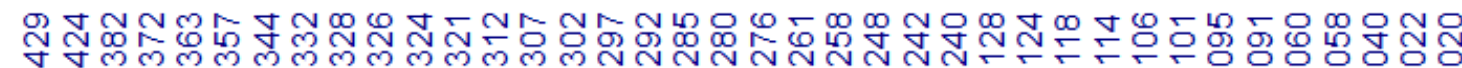

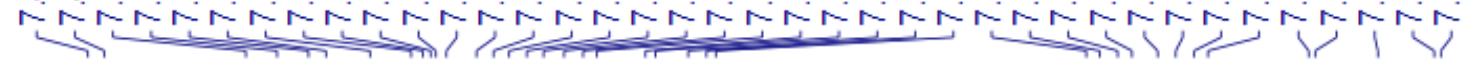
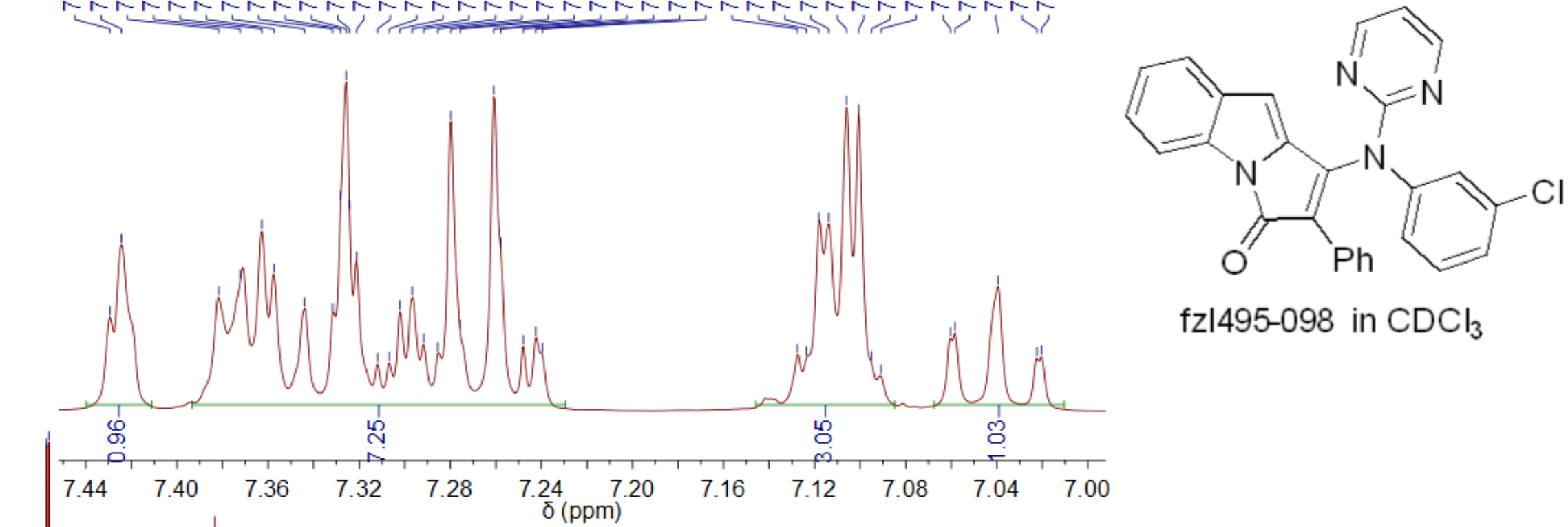

fzl495-098 in $\mathrm{CDCl}_{3}$
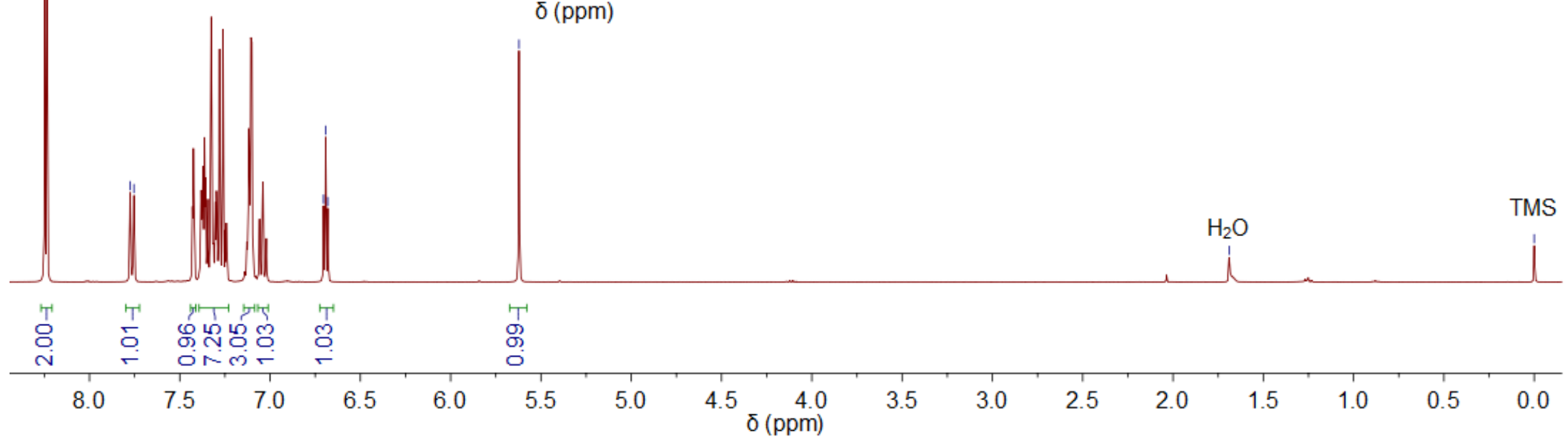

6i $-{ }^{1} \mathrm{H}$ NMR 

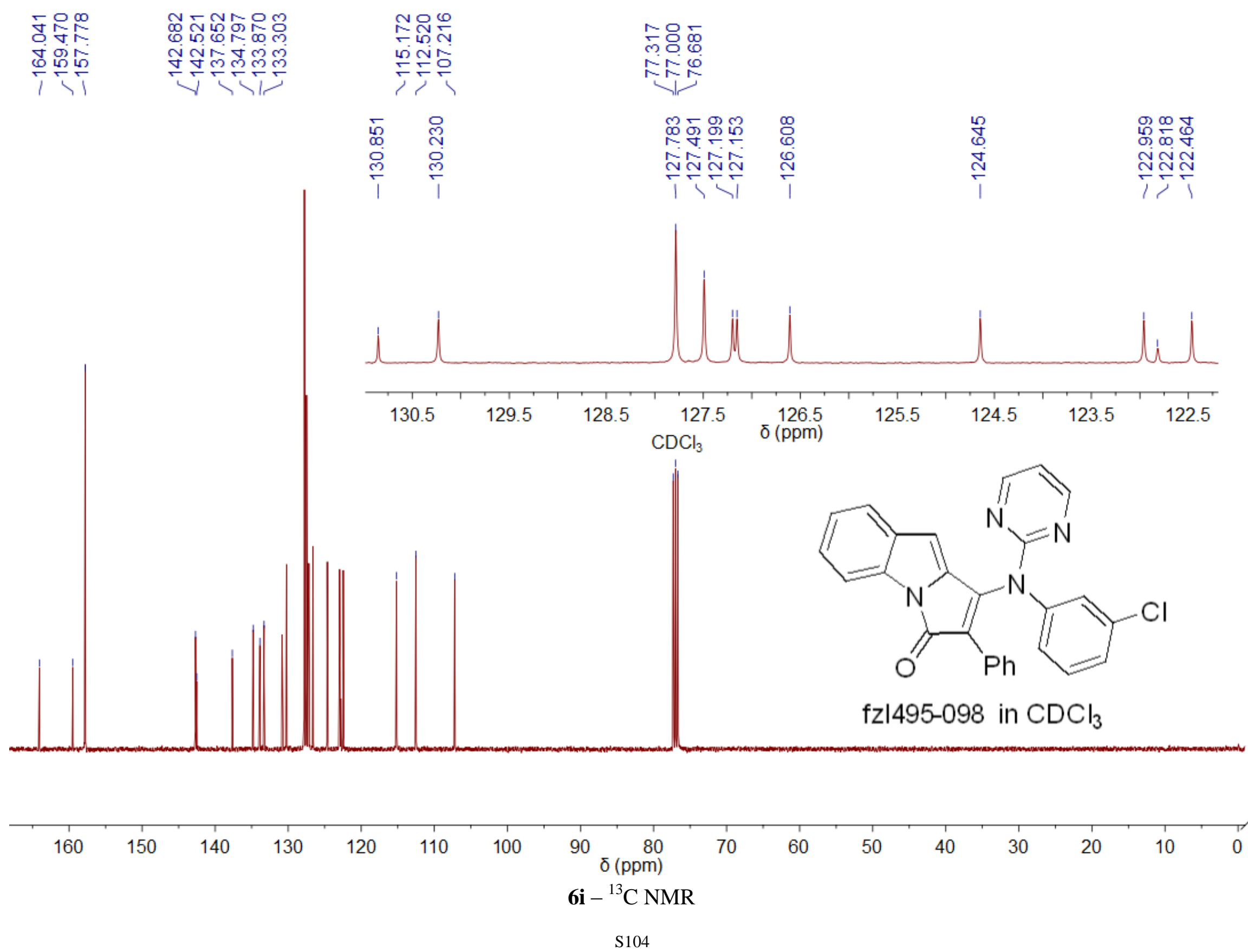

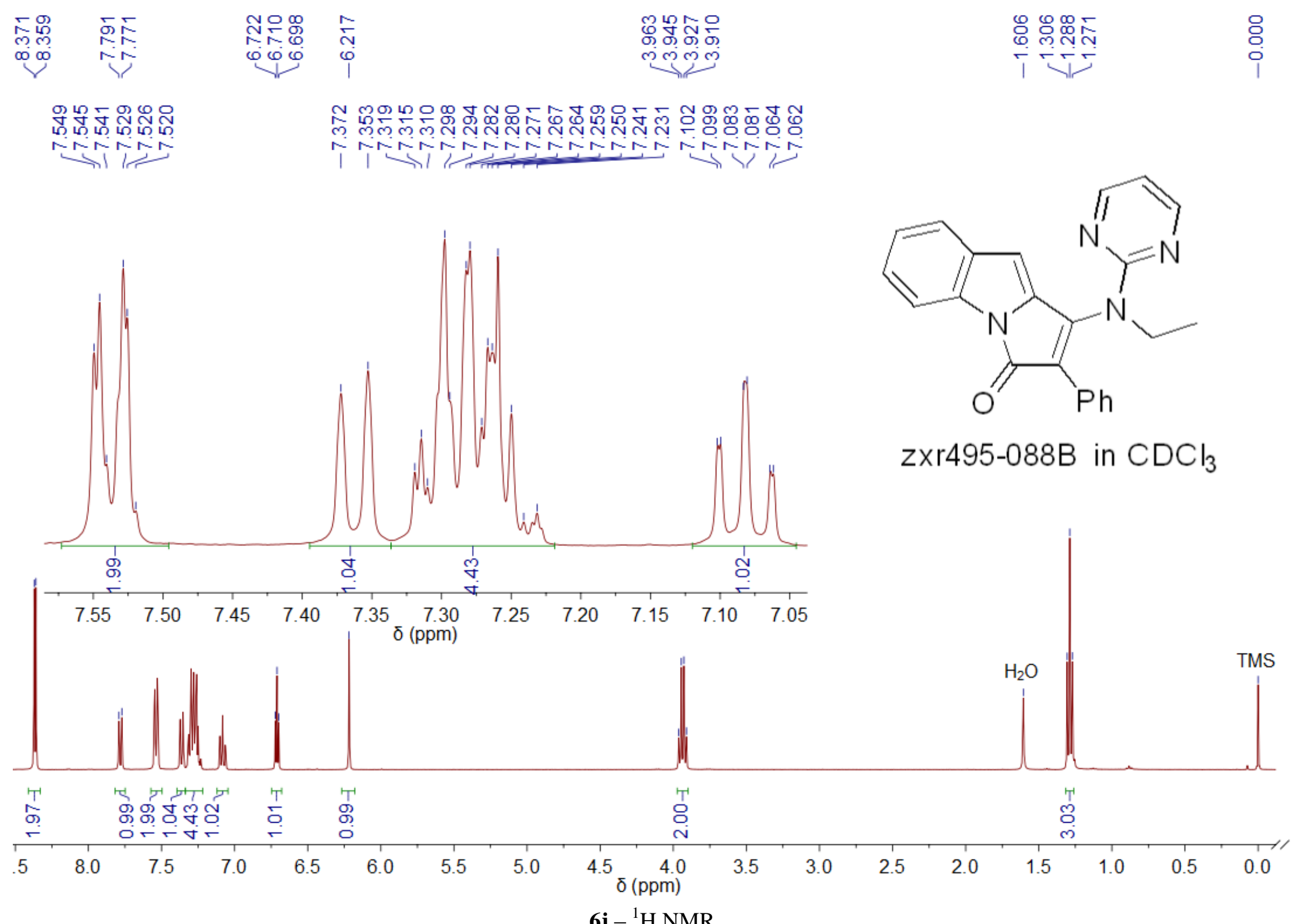

|

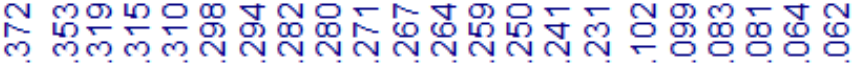

MNMNMMMNMNMNMN

zxr495-088B in $\mathrm{CDCl}_{3}$

6j $-{ }^{1} \mathrm{H}$ NMR 

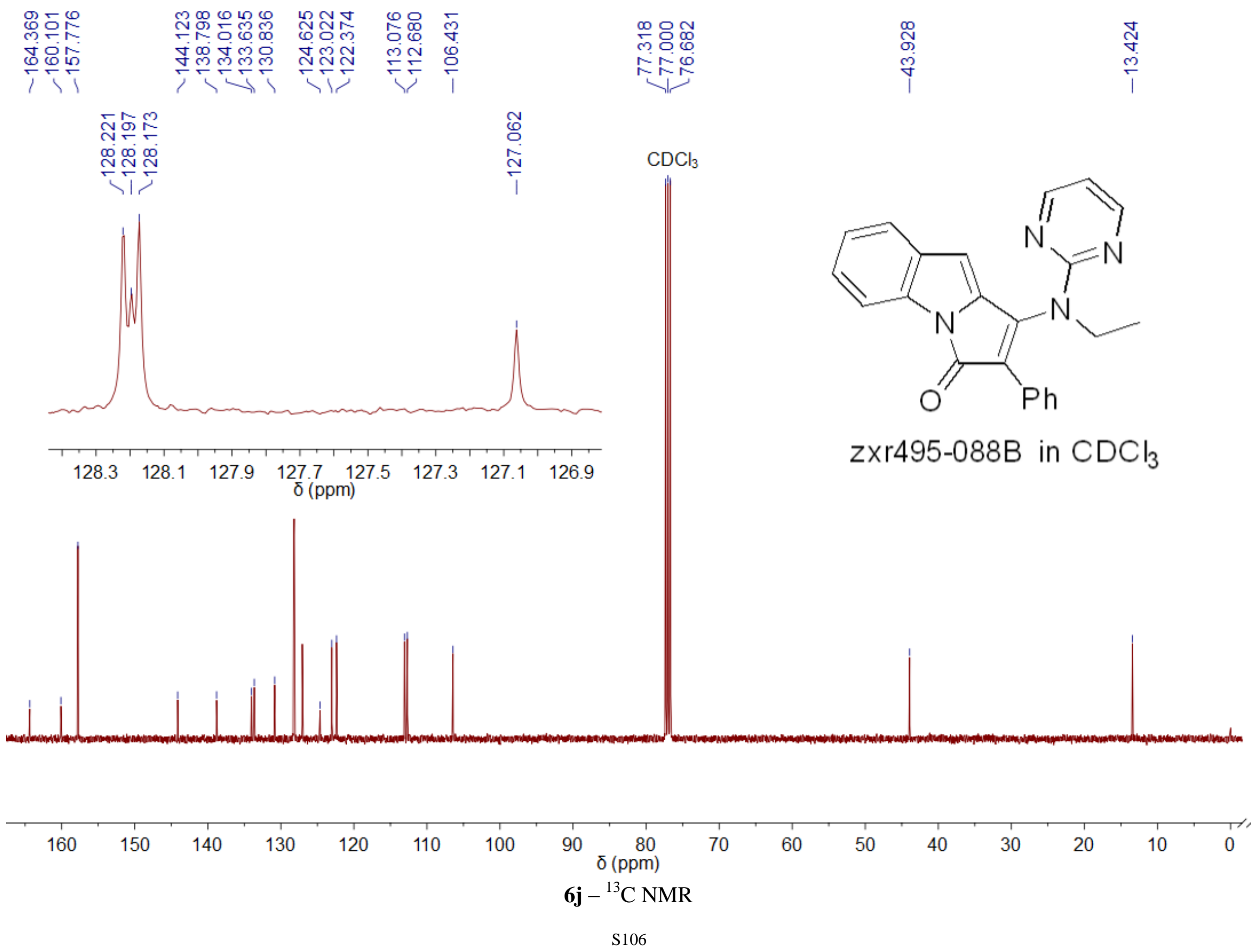


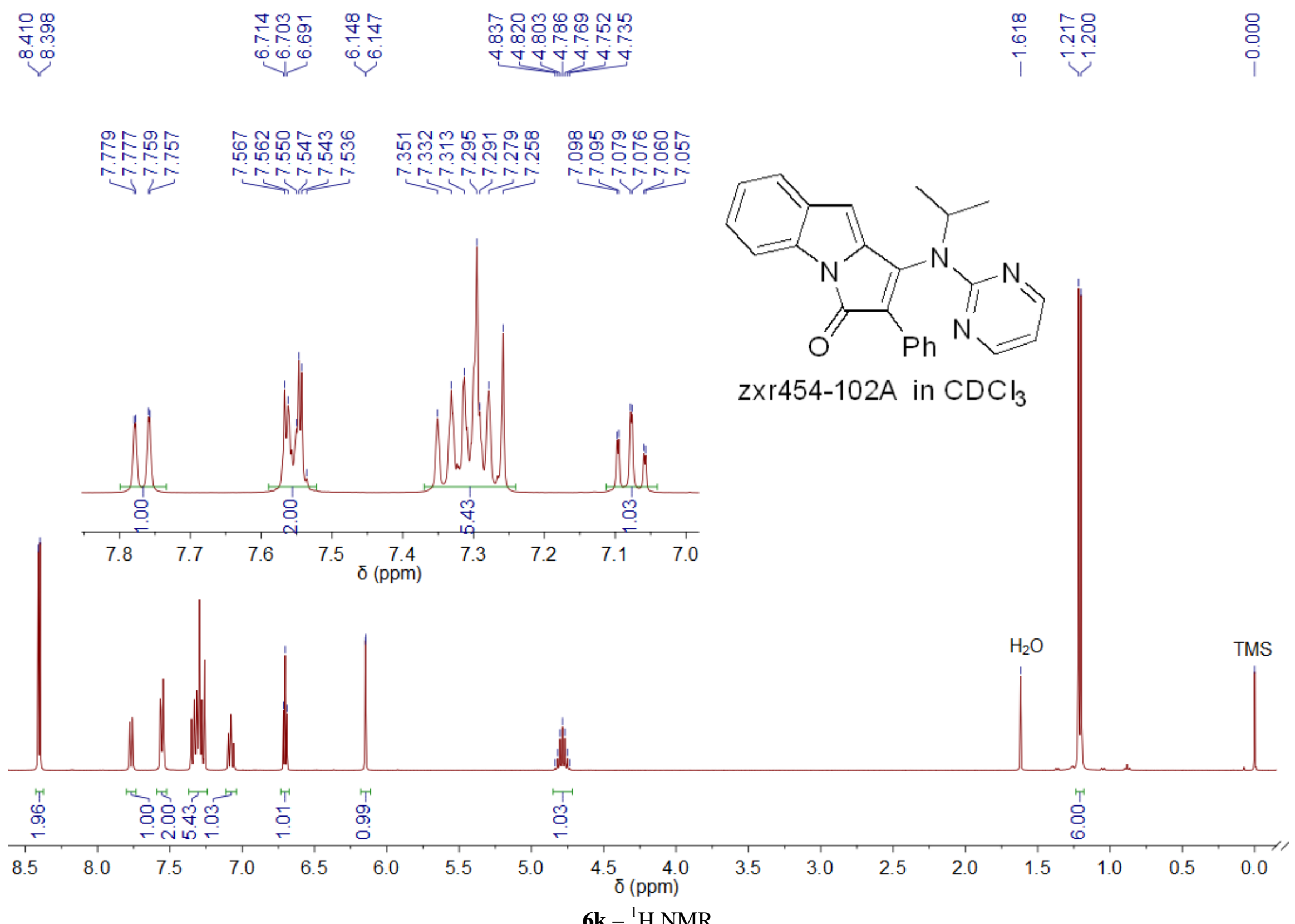




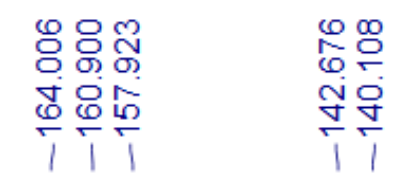

$m$
$m$
$\stackrel{m}{\circ}$

등용

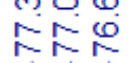

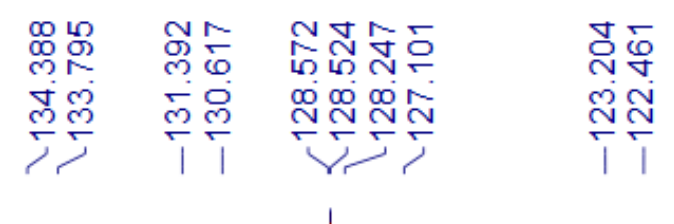

空茛

강

$\mathrm{CDCl}_{3}$
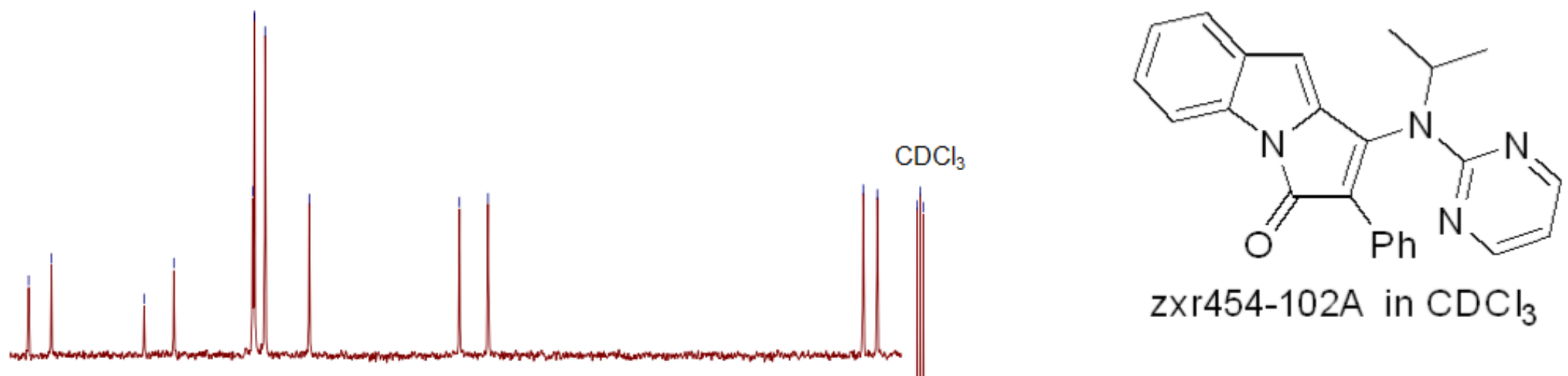

zxr454-102A in $\mathrm{CDCl}_{3}$

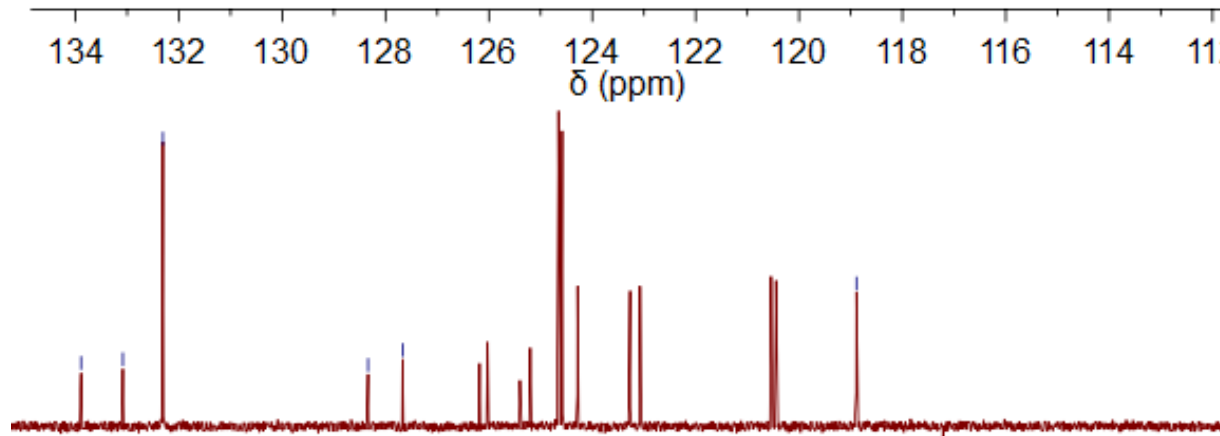

\begin{tabular}{ll}
\hline \\
0 \\
0
\end{tabular}

용

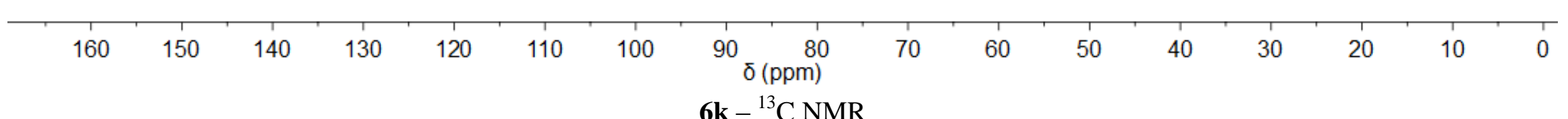

6k $-{ }^{13} \mathrm{C}$ NMR 
赵

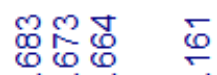

نi

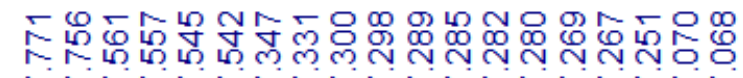

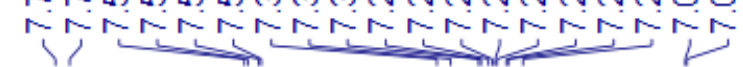
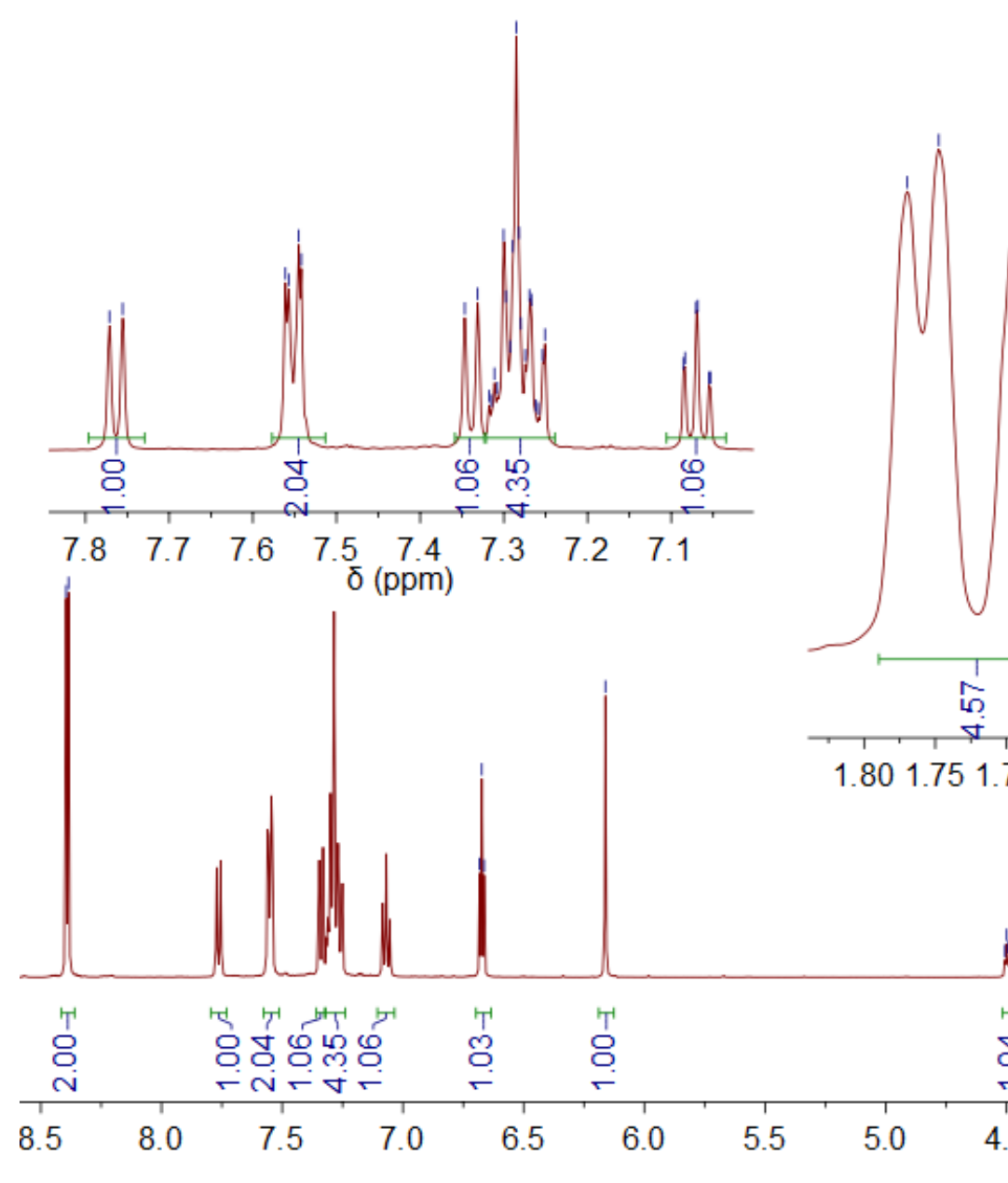

$\log$

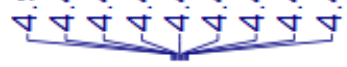

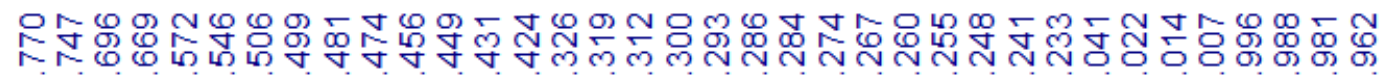
$5-5-5-5-5-5-5-5-5-5-575-5-50909$

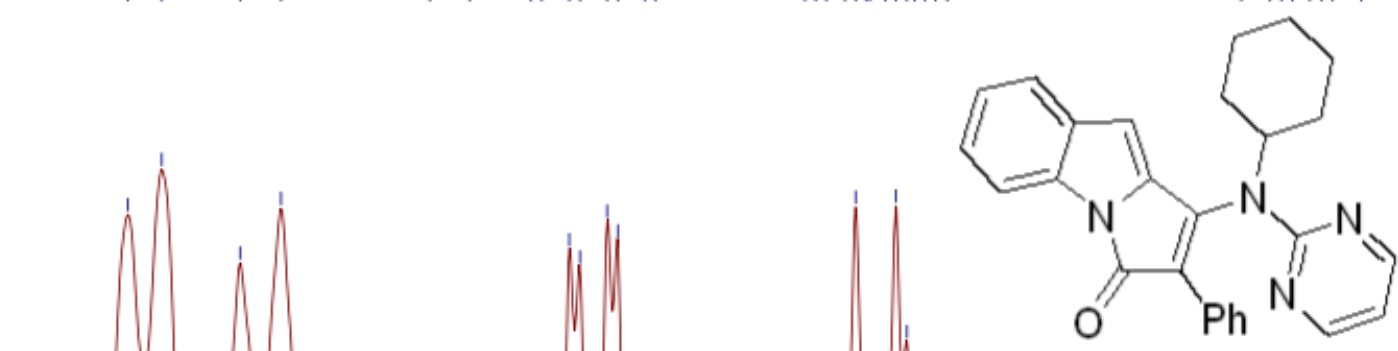
fzl495-082-2 in $\mathrm{CDCb}$

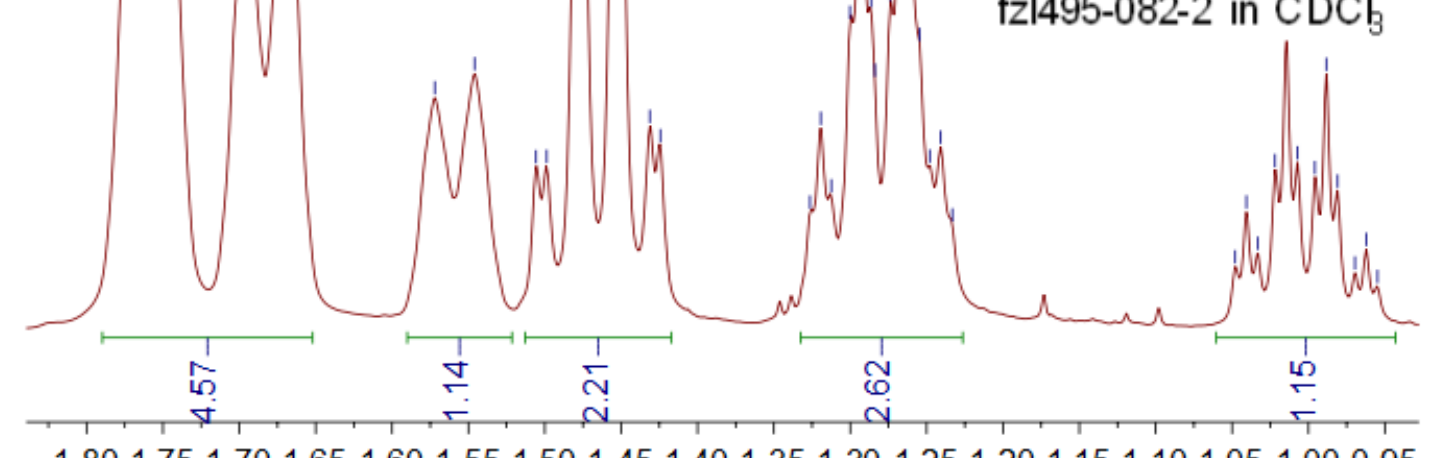
$1.801 .751 .701 .651 .601 .551 .501 .45 \underset{\delta}{1.401 .35} 1.301 .251 .201 .151 .101 .051 .000 .95$
$\delta(\mathrm{ppm})$

\section{.}




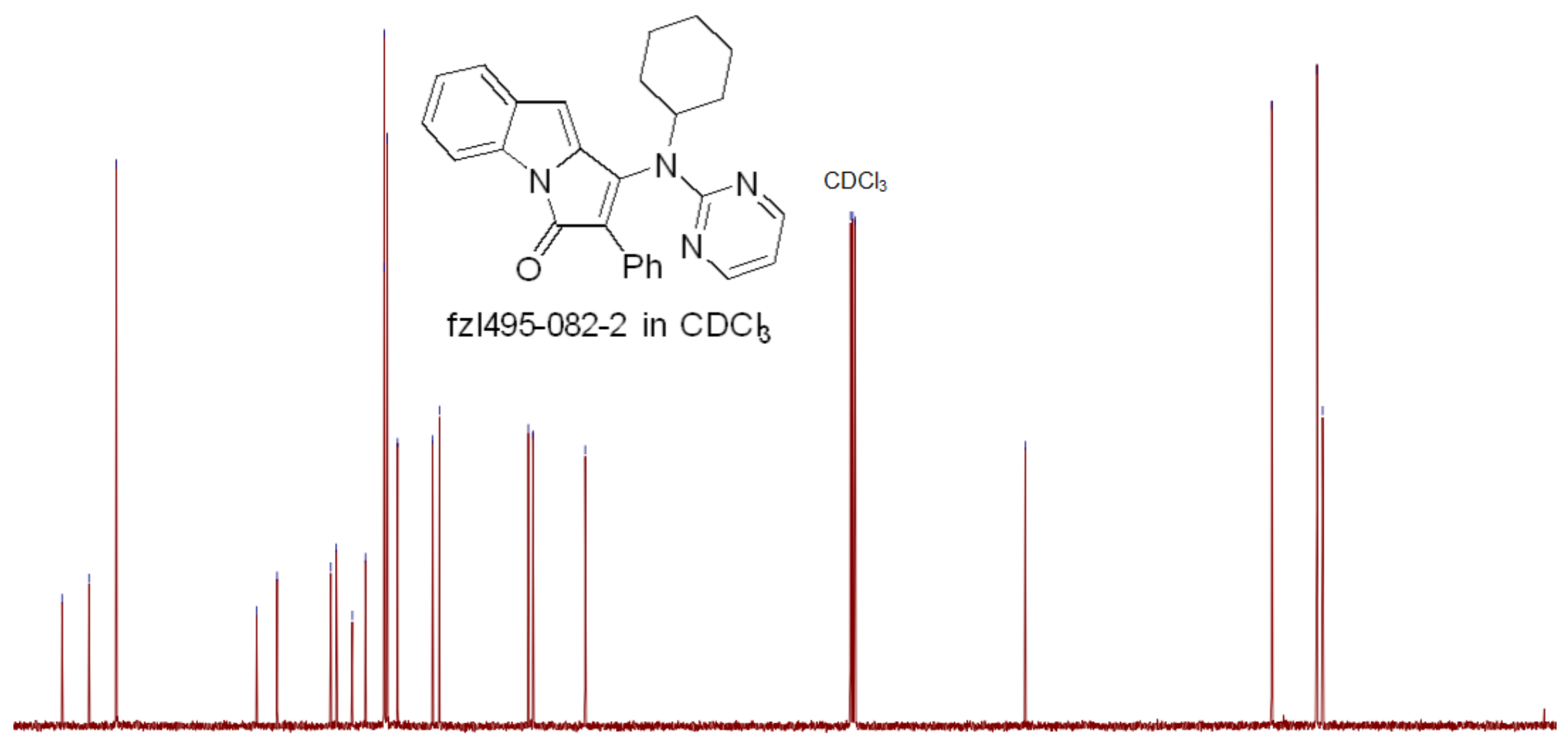

$\begin{array}{lllll}160 & 150 & 140 & 130 & 120\end{array}$

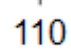

100

$90 \quad 80$

6l $-{ }^{13} \mathrm{C} \mathrm{NMR}$ 
Scheme S1. Proposed Reaction Mechanism

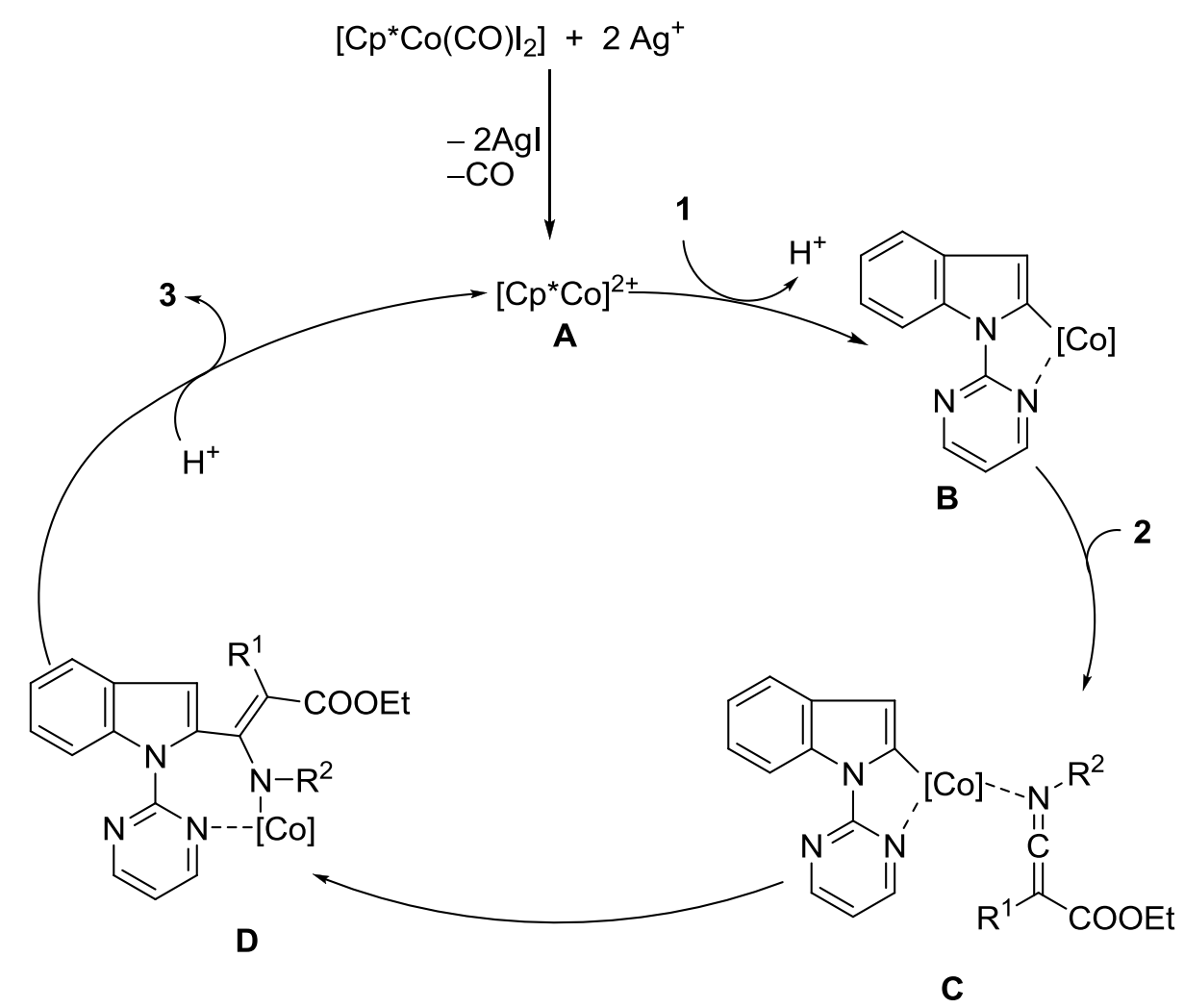

